Florida International University FIU Digital Commons

4-20-2011

\title{
A Methodology to Select an Enterprise Resource Planning System for a Small or Medium Sized Enterprise
}

Richard Burton

Florida International University, mrb001@comcast.net

DOI: $10.25148 /$ etd.FI1 1050602

Follow this and additional works at: https://digitalcommons.fiu.edu/etd

\section{Recommended Citation}

Burton, Richard, "A Methodology to Select an Enterprise Resource Planning System for a Small or Medium Sized Enterprise" (2011). FIU Electronic Theses and Dissertations. 387.

https://digitalcommons.fiu.edu/etd/387 


\section{FLORIDA INTERNATIONAL UNIVERSITY}

Miami, Florida

\section{A METHODOLOGY TO SELECT AN ENTERPRISE RESOURCE PLANNING SYSTEM FOR A SMALL OR MEDIUM SIZED ENTERPRISE}

A dissertation submitted in partial fulfillment of the requirements for the degree of DOCTOR OF PHILOSOPHY

in

INDUSTRIAL AND SYSTEMS ENGINEERING

by

Richard Burton

2011 
To: Dean Amir Mirmiran

College of Engineering and Computing

This dissertation, written by Richard Burton, and entitled A Methodology to Select an Enterprise Resource Planning System for a Small or Medium Sized Enterprise, having been approved in respect to style and intellectual content, is referred to you for judgment.

We have read this dissertation and recommend that it be approved.

$\begin{array}{r}\hline \text { W. Kinzy Jones } \\ \hline \text { Shih-Ming Lee } \\ \hline \text { Ronald Giachetti } \\ \hline \text { Chin-Sheng Chen, Major Professor }\end{array}$

Date of Defense: April 20, 2011

The dissertation of Richard Burton is approved.

Dean Amir Mirmiran
College of Engineering and Computing

Florida International University, 2011 


\title{
ABSTRACT OF THE DISSERTATION \\ A METHODOLOGY TO SELECT AN ENTERPRISE RESOURCE PLANNING \\ SYSTEM FOR A SMALL OR MEDIUM SIZED ENTERPRISE
}

by

\author{
Richard Burton
}

Florida International University, 2011

Miami, Florida

\section{Professor Chin-Sheng Chen, Major Professor}

Enterprise Resource Planning (ERP) systems are software programs designed to integrate the functional requirements, and operational information needs of a business. Pressures of competition and entry standards for participation in major manufacturing supply chains are creating greater demand for small business ERP systems. The proliferation of new offerings of ERP systems introduces complexity to the selection process to identify the right ERP business software for a small and medium-sized enterprise (SME). The selection of an ERP system is a process in which a faulty conclusion poses a significant risk of failure to SME's. The literature reveals that there are still very high failure rates in ERP implementation, and that faulty selection processes contribute to this failure rate. However, the literature is devoid of a systematic methodology for the selection process for an ERP system by SME's. This study provides a methodological approach to selecting the right ERP system for a small or medium-sized enterprise. The study employs Thomann's meta-methodology for methodology development; a survey of SME's is conducted to inform the development of the methodology, and a case study is employed to test, and revise the new methodology. The study shows that a rigorously 
developed, effective methodology that includes benchmarking experiences has been developed and successfully employed. It is verified that the methodology may be applied to the domain of users it was developed to serve, and that the test results are validated by expert users and stakeholders. Future research should investigate in greater detail the application of meta-methodologies to supplier selection and evaluation processes for services and software; additional research into the purchasing practices of small firms is clearly needed. 


\section{TABLE OF CONTENTS}

CHAPTER

PAGE

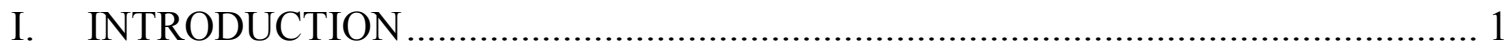

$1.1 \quad$ Background and Motivation..................................................................... 1

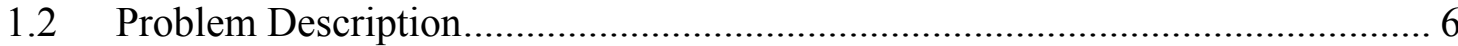

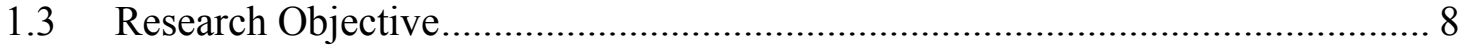

1.4 Scope, Assumptions and Constraints ………….......................................... 10

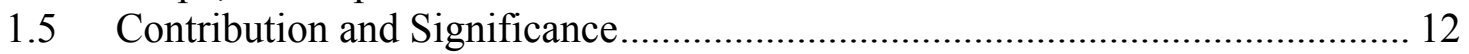

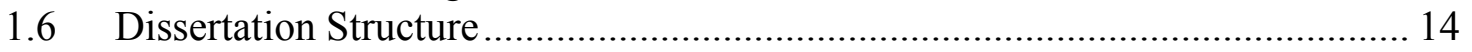

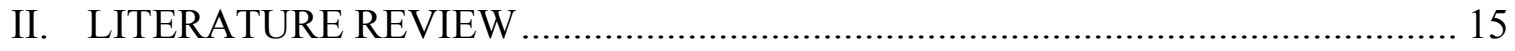

$2.1 \quad$ Meta-methodology …………………………………………………… 15

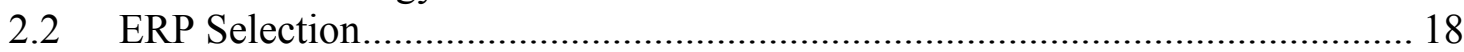

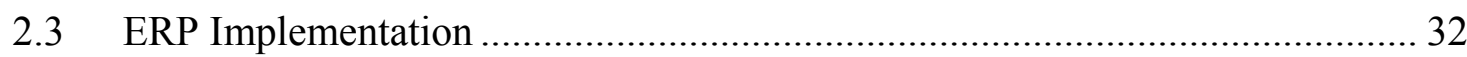

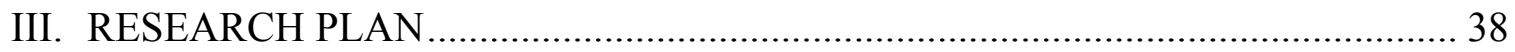

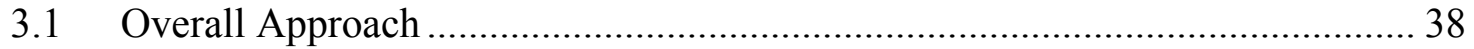

3.2 Detailed Research Plan................................................................................. 40

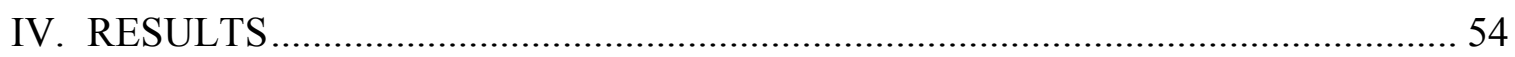

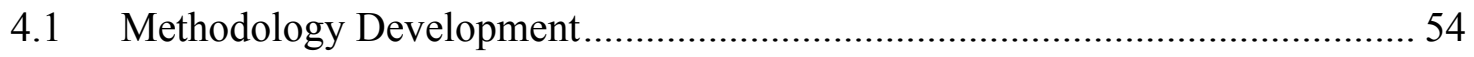

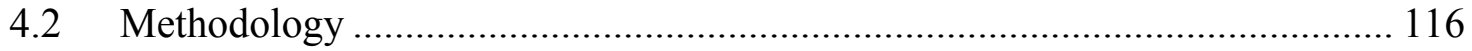

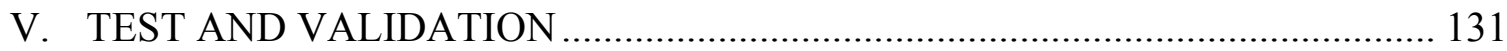

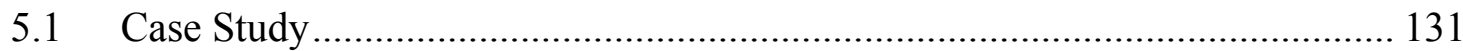

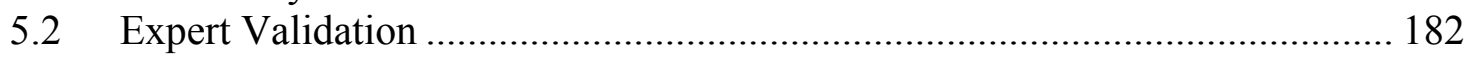

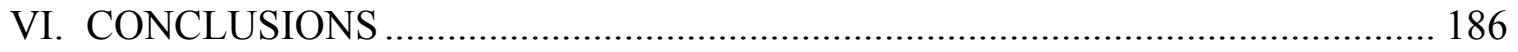

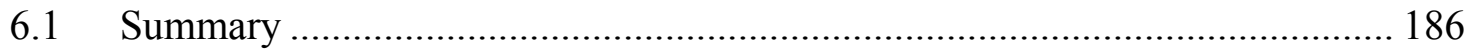

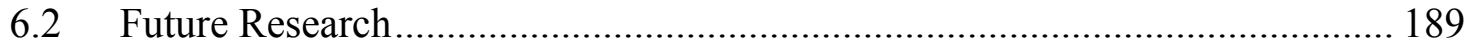

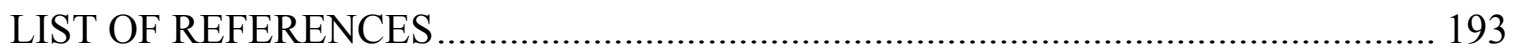

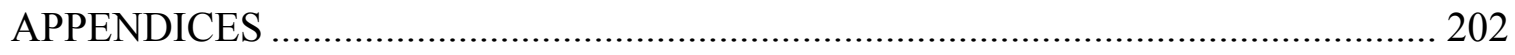

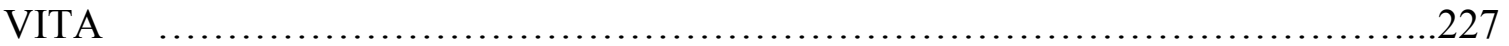




\section{LIST OF TABLES}

TABLE

PAGE

Table 1 - Business Profile of Responding Companies.............................................. 153

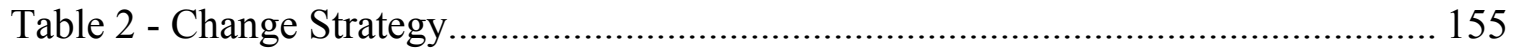

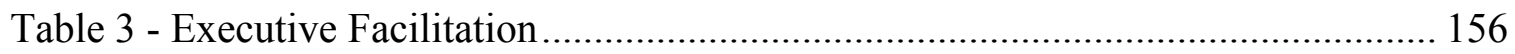

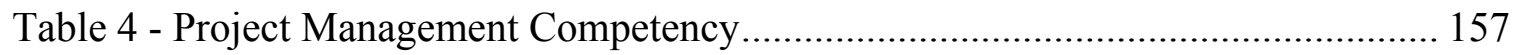

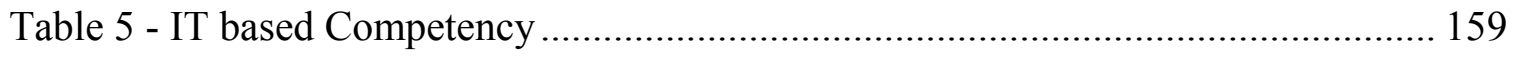

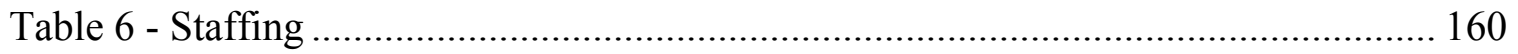

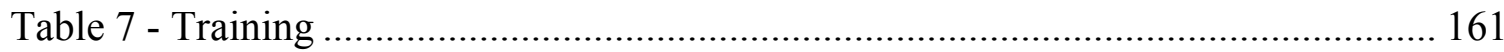

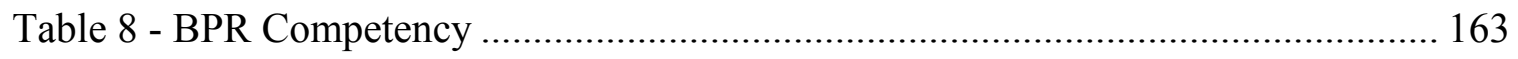

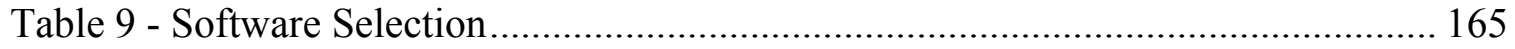

Table 10 - Project Communication and Support..................................................... 166 


\section{LIST OF FIGURES}

FIGURE

PAGE

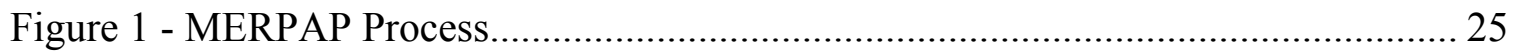

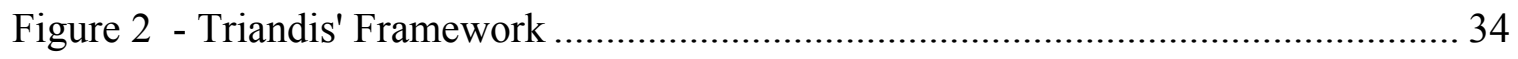

Figure 3 - Flow Chart of ERP Selection Methodology .................................................... 42

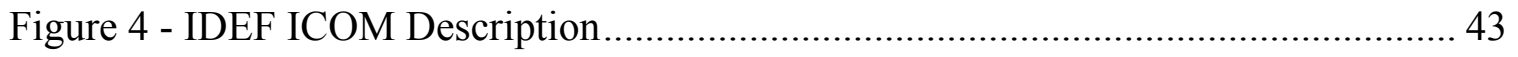

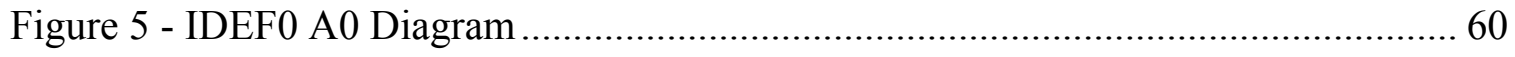

Figure 6 - IDEF0 A0 Select an ERP System for an SME ............................................... 62

Figure 7 - Diagram A1 Review Strategy/ Charter Selection Process ............................... 65

Figure 8 - Diagram A11 Initiate Selection Process........................................................ 66

Figure 9 - Diagram A111 Assign Staff ERP Team...................................................... 67

Figure 10 - Diagram A112 Develop Charter................................................................ 68

Figure 11 - Diagram A12 Conduct Selection Process Chartering Meeting........................ 71

Figure 12 - Diagram A13 Conduct Strategic Briefing and Review Processes ................. 74

Figure 13 - Diagram A131 Perform Internal SWOT Review ………………………..... 78

Figure 14 - Diagram A132 Identify Value Proposition for Selection Process................. 79

Figure 15 - Diagram A133 Conduct Briefing ………………....................................... 81

Figure 16 - Diagram A134 Prepare Selection Project Plan........................................... 82

Figure 17 - Diagram A2 Define and Qualify Strategic and Operational Requirements

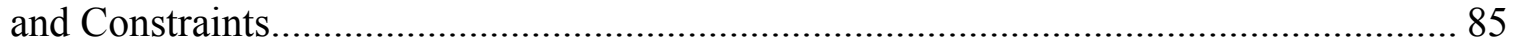

Figure 18 - Diagram A21 Conduct Management Interviews......................................... 86

Figure 19 - Diagram A22 Conduct JRP Planning Activity ……….................................. 88 
Figure 20 - Diagram A23 Determine IT System Baseline Information 89

Figure 21 - Diagram A24 Review, Qualify, Validate and Categorize Requirements and Constraints... 90

Figure 22 - Diagram A3 Identify and Assess Available ERP Systems........................ 92

Figure 23 - Diagram A31 Create and Aggregate List of all Available ERP Systems...... 96

Figure 24 - Diagram A32 Organize and Classify Alternatives .................................. 97

Figure 25 - Diagram A33 Review and Reduce the Number of Alternatives .................. 98

Figure 26 - Diagram A34 Develop and Issue RFI ............................................. 101

Figure 27 - Diagram A35 Qualify RFI Respondents and Audit References................. 102

Figure 28 - Diagram A41 Categorize and Analyze Requirements.............................. 104

Figure 29 - Diagram A43 Develop Performance Test Scenarios................................ 105

Figure 30 - Diagram A44 Develop Scoring Tool and Schedule Vendor

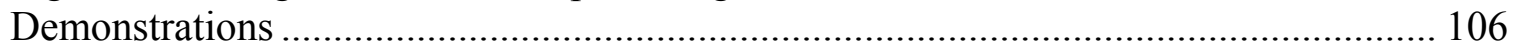

Figure 31 - Diagram A45 Conduct Demonstrations and Score ERP Systems.............. 107

Figure 32 - Diagram A5 Select Best System....................................................... 109

Figure 33 - Diagram A51 Issue RFP for Best and Final Pricing............................... 110

Figure 34 - Diagram A52 Rank Finalist Systems wrt Functionality.......................... 111

Figure 35 - Diagram A53 Calculate Costs of Ownership ....................................... 112

Figure 36 - Diagram A54 Perform Final Selection ................................................. 113

Figure 37 - Core Competencies for ERP adoption. ............................................ 132

Figure 38 - Strategic Considerations for Change Management ................................. 133

Figure 39 - ERP Selection Team and Communication Paths ................................... 134

Figure 40 - Technology Baseline (Network Hardware) 2009 .................................... 143

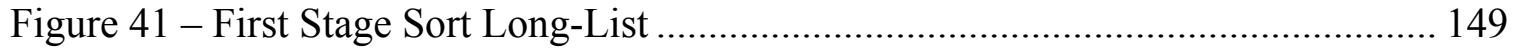


Figure 42 - Sample Requirements Ranking Worksheet................................................. 151

Figure 43 - Finalist ERP Systems Weighted Evaluation Scores...................................... 170

Figure 44 - ERP System Selection Spreadsheet ………………….............................. 171

Figure 45 - Other Requirements performance score matrix......................................... 173

Figure 46 - Bartlett's Sphericity Test Results for Other Requirements ......................... 175

Figure 47 - PCA Information for Other Requirements ............................................. 175

Figure 48 - Scree plot for Other Requirements ........................................................ 176

Figure 49 - Correlation Matrix for Other Requirements ............................................... 177

Figure 50 - PCA Information for Manufacturing Requirements.................................. 178

Figure 51 - Scree plot for Manufacturing Requirements .............................................. 179

Figure 52 - PCA Information for Accounting Requirements....................................... 180

Figure 53 - Scree plot for Accounting Requirements................................................ 180

Figure 54 - PCA Information for Reporting Requirements ........................................... 181

Figure 55 - Scree plot for Reporting Requirements .................................................. 181

Figure 56 - Methodology Validation Survey ......................................................... 185 


\section{INTRODUCTION}

\subsection{Background and Motivation}

Enterprise Resource Planning (ERP) systems are computer software programs designed to integrate the multiple individual functional requirements, and operational information needs of a business. The primary purpose of an ERP system is to establish a central data resource for all the information that is required to be recorded for day to day operations, and to provide that information to the functional elements of the organization as needed to fulfill the goals of the business. They are typically implemented using relational database schema, which allow for the utilization of a central data set in all business process transactions and the retention of all critical business information. One principle advantage, over a business system built up from individual software's, is that they can provide reports which provide an accurate and real or quasi-real time view of the company and its operational condition. Managers can review reports from a single database, which reflects real-time or very near real time information, rather than the frequently contradictory condition assessments derived from several independent data sources.

Early implementers of ERP systems were primarily large business enterprises, and accordingly, the marketplace for appropriate systems was limited to a few large scale software systems such as BAAN, SAP and J.D. Powers (Peoplesoft $\left.{ }^{\circledR}\right)$. The bulk of the decision process to adopt such a system was therefore based in economic decision 
making and identification of the most closely related system in terms of broad based business culture issues. As the supply chain deepened to include strategic partnerships with small and medium-sized enterprises, there was a growing expectation that these small and medium-sized businesses should adopt ERP systems which emulated, and integrated with, the information requirements of the large business partner. This impetus, and that driven by the desire of small businesses to have better and more timely understanding of the status of production, logistics, finances etc led to a high demand for ERP systems that addressed the unique needs of these businesses in a cost effective manner.

The proliferation of new offerings of Enterprise Resource Planning systems; implemented as software applications, or more recently as ASP (Application Service Provider) hosted software, has added an additional level complexity to the selection and best-value decision making process identify the right ERP business software for small businesses. The current market offers in excess of 200 individual software systems, ranging from large scale systems with multi-faceted capability sets, typically aimed at large business enterprises, to software systems aimed at the small business consumer. All businesses and in particular small businesses are faced with this daunting selection of offerings, and consequentially, the selection process is a major challenge to organizations which are about to embark on the implementation of ERP in order to improve their integrated business systems. This selection process is a major challenge to any business the outcome of which may realize either huge potential benefits or create great risks for 
the enterprise. As early as 1998 (Martin, 1998) reported the tardiness of implementation and cost overrun liability at $90 \%$, and later papers reveal that $70 \%$ of implementations "fail to deliver anticipated benefits" (Al-Mashari \& Zairi, 2000). Overall, anecdotal evidence suggests that, even today after years of fit analysis improvements in the ERP selection process, up to $60 \%$ of all implementations are regarded as failures in varying degrees.

The global budget for ERP implementation is projected to exceed \$36 Billion in the next decade. The worldwide market for enterprise resource planning (ERP) systems grew at a $4.8 \%$ compound annual rate, rising from $\$ 16.7$ billion in 2005 to more than $\$ 21$ billion in 2010, according to a study from the ARC Advisory Group Inc., Dedham, Mass. A methodology which reliably identifies an ERP system for a small or medium-sized business enterprise and reduces risk in implementation, will serve to provide practical benefits to industry.

An illustrative definition for ERP is proposed by (Wallace, T.F., Kremzar, M.H., 2001):

"An enterprise-wide set of management tools that balances demand and supply, containing the ability to link customers and suppliers into a complete supply chain, employing proven business processes for decision making, and providing high degrees of cross-functional integration among sales, marketing, manufacturing, operations, logistics, purchasing, finance, new product development, and human resources, thereby enabling people to run their business with high levels of customer service and productivity, and 
simultaneously lower costs and inventories; and providing the foundation for effective ecommerce."

Historically, ERP systems can trace their origins from the development of management and resource utilization theories in the post Second World War period. As new concepts, such as Total Quality Management (TQM) and Statistical Process Control (SPC) were developed and widely introduced, it became evident that the data derived from these new techniques had the potential to provide extended information about the efficiency of the enterprise as a whole and its business posture, well beyond the direct effects of improved product quality. Beginning in the late 1940's W. Edwards Deming encouraged the use of SPC theories and production control techniques in the US; many of which had been proposed by a fellow physicist/statistician Walter A. Shewhart, in the late 1930's. The famous; Plan, Do, Check, Act cycle which Deming advocated, was initially formulated by Shewhart in their joint work on the subject of scientific inference (Shewhart, 1939). Shewhart's work demonstrates an early understanding of the key influence that data have with respect to the success of an organization, and proposes two essential functions/attributes that are addressed by ERP systems:

1. "Data have no meaning apart from their context."

2. "Data contain both signal and noise. To be able to extract information, one must separate the signal from the noise within the data."

Deming was an advocate for SPC based management protocols which helped increase the efficiency of American War industry in the 1940's, and he studied and applied the theories of common and special variation. Deming spent much of the WW II involved in 
the education and training of industrial managers and during this time began to identify some of the key concepts of his fourteen points, most particularly, the critical role of upper management's essential 'buy-in' when resorting to large scale changes in business philosophy. There is a widely held consensus that management "buy-in" and its cultural effect on a company is a key factor in the success or failure of ERP implementations.

It has been suggested that, 'management programs', are subject to a life cycle simply stated as; inception, growth, maturity and decline (Crandall, 2005). However, despite the fears to the contrary, some management systems do have extended utility, and indeed actually evolve into better and more widely applicable systems. The MRP - MRP II ERP development path represents just such an evolutionary process. The key factor differentiating MRP II from ERP systems are in fact platform dependent limitations - had the computing environment been more advanced or consolidated around a common architecture in 1980, MRP II could have remained the dominant system for all enterprise system developments. The 'genetic' similarities of MRP II and ERP are apparent in their common approach to addressing system needs, in both cases the systems are predominantly modular in nature, and the systems rely upon the relational database management system (RDBMS) approach to storing and sharing data. MRP is concerned primarily with manufacturing materials while MRPII is concerned with the coordination of all manufacturing activities, including logistics, finance, and human resources. The goal of MRPII, and of ERP in a broader sense, is to provide consistent data to all decision makers in the manufacturing process as the product is manufactured. 
MRP and MRP II are still in current use either as standalone solutions, or as subcomponents of a more complex suite of management tools which can be described in their entirety as an Enterprise Resource Planning software system. Despite the recognition of the weaknesses of earlier MRP and MRP II systems, and the development of solutions to these weaknesses, some systems have in fact enjoyed prolonged utility. Heritage MRP systems are frequently maintained because they are the 'optimal' solution for a particular enterprise and remain appropriate due to lower levels of need which are consistent with the maturity level of the enterprise.

The wholesale development of business information systems, enterprise systems and ERP systems has led to a highly competitive marketplace. In our research we have identified over 200 individual systems which claim to be ERP's and are available to companies that are considering the introduction of an integrated enterprise software solution. Adopting an enterprise software system introduces a number of cost factors to an organization; cost of acquisition and maintenance, cost of implementation, cost of operational disruption, costs of morale/psychological impacts, and for a small business can introduce the risk of failure of the entire business.

\subsection{Problem Description}

The challenge facing any company is therefore; which ERP system is the "right one" for our enterprise? The problem is frequently referred to as the determination of 'best fit'. At its simplest, the 'best fit' solution may appear to be a process of identifying current 
and future requirements of the company, and ensuring that the baseline capability of the chosen software should, at a minimum, match the efficiency and functionality of existing (non-integrated) systems. Such a solution would be expected to provide the advantages of improved cost control and functional management, which are significant but modest improvements to business efficiency; most companies actually expect far more from the investment in an enterprise system.

The selection of an ERP system is driven by a strategic decision to improve, integrate and adopt more efficient business systems. Typically, a firm identifies weaknesses in its existing systems at a point where constraints to growth or limitations to future expansion of the business are apparent. The business advantage of most small or medium-sized businesses is to be perceived as a lower cost, more adaptable and responsive than larger manufacturers. In light of this approach, many small and medium-sized businesses seek to align their business planning with the major projects of larger manufacturers, effectively becoming 'preferred providers' of component parts and products for the larger manufacturer. One of the implications of adopting this kind of alignment strategy is that the larger manufacturer's supply chain qualification system will usually specify that the small supplier be able to feed production and cost data to the large business in quasi-real time. For most small companies, such data can only be produced by adopting an ERP system. The specific measures of merit for future candidate systems are therefore based on strategic needs to maximize profit through increased efficiency in operations, management, production, design etc. Since, an ERP approach fundamentally involves the integration of a number of diverse work systems, through a common RDBMS, 
changes in business processes are often intrinsic components of the broad changes that the company must undertake. In addition, based on the degree of fit, a measure of compatibility of ERP software to the required business processes, there may exist a change process by which the software itself has to be adapted to a preferred existing process.

Each of these activities is a component of the cost drivers for the implementation of a new ERP system. The degree of disruption and cost of the change process from an existing business process environment to one with a working ERP, is the key concern in making the correct trade-offs and decisions with respect to the selection of a new ERP system that is best suited to the company.

\subsection{Research Objective}

The primary objective of the research will be to produce a methodology which may be implemented as an ERP system selection tool by SME's. By design, the methodology will relate expert knowledge, best practices and current knowledge of success factors in ERP implementation and will resolve a selection decision for the ERP system that will provide best value to the company.

The selection of an ERP system is driven by a strategic decision to improve, integrate and adopt more efficient business systems. Typically, a firm identifies weaknesses in its existing systems at a point where constraints to growth or limitations to future expansion 
of the business are apparent. The specific measures of merit for future candidate systems are therefore based on strategic needs to maximize profit through increased efficiency in operations, management, production, design etc. Since, an ERP approach fundamentally involves the integration of a number of diverse work systems, through a common RDBMS, changes in business processes are often intrinsic components of the broad changes that the company must undertake. In addition, based on the degree of fit, a measure of compatibility of ERP software to the required business processes, there may also be a change process by which the software itself has to be adapted to a preferred existing process. Each of these activities is a component of the cost drivers for the implementation of a new ERP system. The degree of disruption and cost of the change process from an existing business process environment to one with a working ERP, is the key concern in making the correct trade-offs and decisions with respect to the selection of a new ERP system that is best suited to the company. It is important to a small business that the ERP system that is selected must have good alignment with its existing processes and needs, and that customization is kept to a minimum, to preserve funding. It is also important that the evaluation of the ERP system be understandable and has direct application or are highly parallel to their own performance metrics.

In order to have broad applicability, a survey of best practices was conducted, to identify the methods and procedures adopted by small and medium-sized businesses in achieving successful selection and implementation of ERP systems; and to identify the common modes of failure, perceived failure and critical weaknesses in the ERP selection and implementation in this class of business. The research is designed to evaluate and 
integrate/differentiate the risks associated with the foregoing factors and propose a lower risk ERP selection methodology which incorporates the understanding of the interrelationship of the selection process and the implementation of new ERP systems.

We intend show that it is possible to resolve answers to complex multi-variable, mixed qualitative and quantitative criteria problems, by means of a rigorous methodological approach that is effective, low cost, and user friendly - attributes that will make the tool valuable to small and medium-sized businesses in their supply chain decision making processes.

Lastly, we validate and verify the results of the proposed methodology; the experiences of a small business selecting an ERP system using the selection methodology will be assessed. Such validation will serve two purposes; firstly, that the meta-methodology approach is a viable way of creating selection tools that may be applied to supplier selection problems in industrial and other settings; and secondly, that the methodology itself is acceptable to experts in the field and is qualified for application to the real world purpose of the tool.

\subsection{Scope, Assumptions and Constraints}

The methodology was designed to be applicable to SME business entities. While, the entire process of adopting an ERP system is under a great deal of academic study the scope of this work is bounded by the activity space which is related to; the selection of an 
appropriate ERP system for a small or medium-sized enterprise. The selection process is necessarily influenced by external factors, such as marketplace dynamics (ERP vendors for the small and medium-sized consumer, come and go, and their offerings are updated continually). Planning and preliminary actions and decisions associated with the whole continuum of the activities of ERP implementation are largely outside the scope of this study.

ERP vendors in the SME market arena are increasingly promoting their software with a concentration on the revenue they can achieve by customizing their products to the processes that are in place with the customer business. This approach is an expensive one, and may often be beyond the means of a small business. Consequently, many small businesses defer adopting ERP systems because of the budgetary constraints and large commitment of personnel resources that ERP introduction demands. We assume that the SME targeted by the proposed methodology, has reached a higher organizational maturity level which characterizes the desire for more sophisticated organizational management tools like ERP systems. We also assume that the SME has in parallel achieved process and procedural efficiency, and is well informed about its own internal process architecture and is self-aware in respect to its advantages and disadvantages in the marketplace. The adoption of an ERP system is a component of a much larger commitment for any company. This commitment involves the adoption of re-engineered processes, extensive formal training, the redefinition of quality metrics, and a variety of initiating activities, such as formalized business process knowledge and careful budget allocation for the overall objective of implementing an ERP system. A large proportion 
of small businesses have recognized the potential of ERP technology but are constrained from adopting the systems because of the uncertainty they experience in understanding the total cost of ownership for these systems. ERP costs are frequently hidden to the client who has a less than expert knowledge of the total cost of ownership; while, acquisition costs may be openly reported, the longer term costs including upgrades, maintenance and management fees are not always transparent.

\subsection{Contribution and Significance}

By applying a methodological approach we contribute to the field of ERP studies by providing a new tool which can facilitate better decision making in the selection of ERP systems by this class of business. Since, there is no existing methodology which addresses the ERP selection process for SME's the development of this tool is an important first step in providing a clear and prescriptive path to the selection of a reliable ERP system.

The competitive pressures for small businesses are enormous, yet they are regarded as the critical to the economy. According to the US Census Bureau, 2006 data, there are 23 million small businesses in the US, and they employ more than half of all workers. A methodology which provides an easily understandable process for ERP selection; offers some degree of certainty in understanding the total cost of ownership; and sheds light on the issues relating to the risks of implementation, will help greater numbers of SME's to adopt the technologically advantageous ERP systems. By selecting the right ERP system, 
SME's can improve their competitive posture and gain new market share by being able to participate in electronic document exchange with large business partners, who regard this capability as a legitimate standard for entry in their supply chains.

The selection of an ERP system is a multi-attribute decision process, which consists of quantitative and qualitative elements; the methodology developed in this study provides a transparent, participative process which is easy to access for all levels of staff experience and knowledge. While the methodology is developed with evaluation criteria that are identified by the SME, and are referenced to its own performance metrics, the survey element of the methodology development assists in putting the thrust of the selection process in alignment with benchmarked standards of its completion.

The application of a methodology to this problem builds on the principles of system engineering by applying integrated methods to the underlying sub-problems of the activities required to operationalize the purpose of the methodology. A metamethodology allows us to decompose the problems which exist within the purpose. This decomposition is achieved by using systems engineering tools like IDEF0, or commercial process decomposition software to identify the activities and the tools that are required to produce viable methods to resolve the activities into inputs for succeeding stages of the methodology. We propose a suite of individual tools, techniques and procedures which systematically breakdown the challenges into smaller or more rational elements, and allow us to progress through the methodology's steps and carry forward the right sets of information and output which make the final selection process possible. 
The development of the new methodology also provides an additional validation for the meta-methodology approach to resolving real world problems in an effective and systematic way.

\subsection{Dissertation Structure}

This document presents a brief outline of the commercial context of the development of ERP systems; a description of the problem of selecting an ERP system for a small business, and its relevance to academic interests. We provide a synopsis of the research objectives, scope and the assumptions and constraints pertaining to the research, and propose the contribution and significance of the research. The study continues with a full review of the published academic work with regard to; Meta-methodology and its application, ERP selection, and ERP implementation. The research plan is outlined, and we introduce both the particular and general methods, processes and procedures we will use to build the proposed methodology. Having executed the research plan, we explain and present the results of the process, and details of the methodology development; we evaluate and incorporate the findings of a survey into the proposed methodology and provide the finished methodology for the reader. We test the methodology, through application, with a small business, and demonstrate the operability and practicality of the methodology; we validate the methodology by an expert review process. Finally, we

review and summarize the study outcomes, and present some future research paths that may be instructive and contribute to the academic interest in ERP systems. 


\section{LITERATURE REVIEW}

The literature review which follows is divided into three primary areas of consideration;

firstly, a review of meta-methodology approaches that may inform the development of a methodology for the selection of an ERP system for a small or medium-sized enterprise; secondly, a review of the body of work addressing attempts at developing methods, techniques and procedures for selection of ERP software for any size enterprise, including a search for methodologies which address ERP selection for small and medium-sized enterprises; and lastly, a review of the broad contextual background to ERP implementation issues, covering the current understanding and consensus issues within the body of ERP related research.

\subsection{Meta-methodology}

In order to develop a methodology, consideration must be given to the discussion of the validity and breadth of application of meta-methodologies and their relevance to questions of scientific importance. One of the earliest expressions of the concept of a meta-methodology is attributed to Imre Lakatos (1971) in a critical appraisal by Husain Sarkar (1980). Lakatos' use of the term related to his approach for determining the relative merits of theories of the scientific method. Sarkar deconstructs the form of a methodology into four components; the objective component, consisting of the key definitions of a methodology; the normative component, which bounds the process of appraisal for competing methods within the methodology; the empirical component, which supports the methodology based on findings of other research or scientific 
theories; and an illustrative component, consisting of practical examples which illustrate the efficacy of the methodology.

Thomann (1973) proposed a clarification of the differences between a method and a methodology by providing brief definitions for each; for Thomann, methods are defined as "rules of thumb", procedures or guidelines that guide someone to achieve a given purpose. Sarkar refers to these as heuristics and advice. For Thomann, methodologies are described as a series of operational steps, which define a specific and defined purpose. His clarification points out that a method only provides a general direction for accomplishing a task; while a methodology is more prescriptive of the procedures to be utilized, the sequence and effects sought, and the route that must be undertaken to achieve the desired goal. Thomann also deconstructs the development of methodologies into a series of seven defined steps, with three objective conditions that must be met. It should be noted that Thomann's meta-methodology approach is flexible in that, while a sequential progression of methods and procedures may be called for, the approach also allows for concurrent and optional steps which achieve the same underlying purpose. Such flexibility accommodates the many real world situations where time and resource management issues can only be managed by distributed effort, and the methods composing the methodology require recursive or iterative procedures.

The meta-methodology proposed by Thomann (1973) is a concise and practical armature for development of a new methodology; he attributes the research to collaboration with $\mathrm{T}$. Hutchinson, with synthesized contributions from J. Fortune, and R. Coffing. 
Thomann's meta-methodology proposes seven steps to evolving a new methodology, with the aim of addressing three objectives; determining the purpose, development of all the steps that are contained in the methodology, and testing the methodology to ensure it accomplishes the purpose. Thomann's complete meta-methodology is appended to this study.

In a later work, (Hutchinson, 1984) Thomann's collaborator provides a modified metamethodology which proposes eight distinct steps:

I. Preparation process

II. Choice of problem area

III. Choice of a purpose for the methodology

IV. Test for acceptability

V. Analysis of the implications of the purpose for the general steps that the methodology should contain

VI. Production of an operational definition of the purpose of the methodology

VII. Production of the detailed operational steps of the methodology

VIII. Performance of research on the methodology in order to identify problems and correct them. Although Hutchinson's approach does expand the scope and domain of the development meta-methodology, his meta-methodology does little to enhance the clarity of purpose and in many ways simply reiterates Thomann's meta-methodology approach without additional advantages. 
Gary Holt (1998) provides a review of contractor selection methods and provides a useful analysis of the advantages and disadvantages of competing decision methods. He points out that bespoke approaches (ad hoc tailored methods) are prolific in industrial use but are highly subjective, and therefore not generalizable. He also concludes that methods employing utility ranking, are worthy of further study, and have the characteristic that they facilitate ordinal ranking of alternatives and therefore complete selection. Most of the other methods result in groups of qualified candidates but are unable to differentiate a single leading candidate.

\subsection{ERP Selection}

In their survey of critical success factors for ERP implementation, Hong and Kim (2002) suggest that while organizational fit is recognized as an important selection factor,

"Many vendors... ignore the ...concept, and urge blind trust on ERP", they also point out that there is no empirical confirmation that organizational fit and ERP implementation success are validated.

$\mathrm{Wu}$, in evaluating the pre-implementation factors contributing to successful ERP implementation, proposed two aspects for careful consideration during the selection process, compatibility between the software/hardware and the company's needs; and ease of customization of the software (Wu, 2008) 
Bernroider and Koch (2001) indicated that the selection process adopted by small or medium size businesses is differentiated from the process at large business enterprises. Specifically, they outline four classes of staff participation in the selection process, and that small enterprises are more likely to engage in a participative and inclusive process to select a software system. Their findings supported earlier findings that suggest that participation of the people directly affected by the chosen system, leads to better decision making (Hammer, 1993) (Davenport T. H., 1993), and that consequently the acceptance of the system over the long term is more likely (Guha, 1997). Bernroider and Koch also indicate that; small firms are highly cost conscious, they are more likely than large firms to adopt formal methods of selection and that static economic analysis prevails over time value methods; but that the total numbers of staff involved in the selection are likely to be much lower. The researchers provide valuable underlying advice on the levels of participation, and of the intrinsic characteristics of small and medium-sized businesses which should be observed in formulating a comprehensive selection methodology.

One frequently cited foundational work referenced in the literature is an attempt to characterize processes to facilitate the selection of competing computer (software) projects (Buss, 1983). Buss proposes that the factors that are suitable for prioritization of the selection of computer projects, can be grouped into four distinct categories; financial benefits, business objectives, intangible benefits (among these being; better information presentation, improved decision support, and fulfillment of operational needs), technical importance. He also makes the case that the user community should decide the priorities, 
and that both operational and IT managers should jointly define priorities, and suggests a role for 'steering committees. Buss proposed a method for grid evaluation and ranking schema for the benefit structures he recommended. Interestingly, Buss makes the assertion that many of the critical ERP implementation success factor criteria, recognized in later work, are "intangible". The value of Buss' work is foundational to understanding the complex mix of decisions and activities that form the basis for ERP selection, yet he does not introduce a clearly defined path, nor does he propose the sequence or type of activities that are required in resolving these decisions.

An opinion closer to today's consensus, is voiced by Hallikainen et al. (2004), who propose that; the perception of ERP introduction as a technological initiative' will lead to the failure to realize many of the corollary potential benefits which occur due to reengineering and enhancing the business processes.

Rao (2000) suggests the use of the decision tree model to help with the determination of whether a company should 'buy or build' its ERP system. Rao suggests a fairly simple domain of decision making that can be resolved through a straightforward decision tree.

The four component options from Rao's perspective are; first, design and build a custom ERP system using in-house resources. Clearly, this would require particular expert knowledge and a large resource commitment of specialized skills. Second, perform custom integration of the existing group of systems, using middleware and common data architecture strategies. This approach is a major resource commitment requiring 
specialized skills. Third, purchase an off the shelf option; having sufficient operational fit to the required functionality. This is the option most preferred by businesses in this industry sector, even though there may be costs associated with software adaptation or alternatively BPR needs for the company. Fourth, custom build the software using outside resources. Rao addresses the first elements of the decision process required in identifying whether a company is ready to advance to an integrated ERP system, and deals primarily with the "buy or build" question, clearly the entire selection process is likely to be far more complex.

By the year 2000, research work had focused more precisely on the methodologies required to evaluate ERP projects. Proceeding from a viewpoint that the selection of an ERP system requires multi-dimensional evaluation criteria Teltumbde (2000) introduced a process framework which incorporated learning and decision making processes based on Nominal Group Theory (NGT). He defines ten high-level multi-attribute variables which are tested in a brief case study to demonstrate the outcome of the selection process, applied to three candidate systems, using a comparison of alternatives by the Analytical Hierarchy Process (AHP). Other quantitative investigations have looked at methods to help with elements of the selection process, but like Teltumbde they fail to address the processes, pathway and validation of the selection criteria prior to evaluation; this defect may be addressed by the application of a methodological solution. 
Several researchers feel that "no single ERP system" can address all the functional requirements and business needs that a company may identify (Wei, Chien, \& Wang, 2005) (Teltumbde, 2000) (Hong \& Kim, 2002).

Although, there have been some recent approaches at defining the quantitative relationship of business practices and the operational characteristics of business enterprises, to assist in the selection process for ERP systems, there appear to be no currently available studies which link the comprehensive body of company requirements; business, strategic, functional, and IT etc., to the selection process. While the "fit" of an ERP system to a company has been extensively explored regarding its implementation effects, no study adequately addresses the fitting of specific requirements to ERP capabilities as a basis for software selection.

Much of the substantive research work related to the ERP selection process finds its genesis in studies which look at the more general problem of Information System (IS) selection. Notwithstanding Hallikainen's cautionary commentary regarding the defects that may occur in perceiving ERP as solely an IS problem, the body of ERP research has leveraged several IS studies.

Santhanam and Kyparasis (1995) employ a non-linear model which considers the interaction of factors, and the interdependent facets of alternative IS projects. Scniederjans and Wilson (1991) proposed that IS project selection could be adequately 
accomplished by using a combination of goal programming and AHP. Proceeding from this basis, other researchers (Lee, J.W., Kim, S.H., 2000) suggested that the criteria addressed by Santhanam and Kyparasis were too narrow, and that the combination of zero-one goal programming (ZOGP), and Saaty's Analytic Network Process (ANP) (Saaty, 1999) would provide a more substantial evaluation of alternatives for IS project selection.

Applying these study's findings to the ERP selection process, Wei, Chien, \& Wang (2005) are critical of the scoring method employed by Lucas and Moore (1976) for IS project selection, and propose a selection framework using a fundamental-objective hierarchy and means-objective network attributed to Clemens (1996), as the input basis for the selection method. They employ Saaty's Analytical Hierarchy Process (AHP) (Saaty, 1980) and demonstrate its use to determine which of three ERP vendors most closely matches the fundamental and means objective criteria. The pair-wise comparison, required by the AHP technique, relied upon the decision makers of the company in the author's case study. They admit that the," comparison was limited by their knowledge, experience, and ...cognitive biases". Consequently, extensive training with regard to the AHP scoring process and its strengths and limitations was required.

Citing the intrinsic ambiguities and imprecision resulting from human judgments, Lien and Liang (2005) characterize the ERP selection process as one which is a fuzzy multicriteria decision-making problem. Accordingly, they adopt a project management 
approach to selection. They employ McCall's software quality model (McCall, J., Richards, P.,and Walters, G., 1977) as the basis for the criteria for selection of an ERP system, and apply fuzzy logic techniques to the AHP (FAHP) process to help deal with the defects in the evaluation process that are attributable to linguistic interpretation. They illustrate their methodology by applying it to the selection of an ERP system for a small college, selecting from three vendors.

Verville suggests a disciplined selection process to a manageable level, known as MERPAP (Verville, 2003). Verville's model is drawn from conclusions derived from a fairly small sample set of industrial case studies in which survey data identifies the six common themes for the buying process; Planning, Information Search, Selection, Evaluation, Choice and Negotiation. 


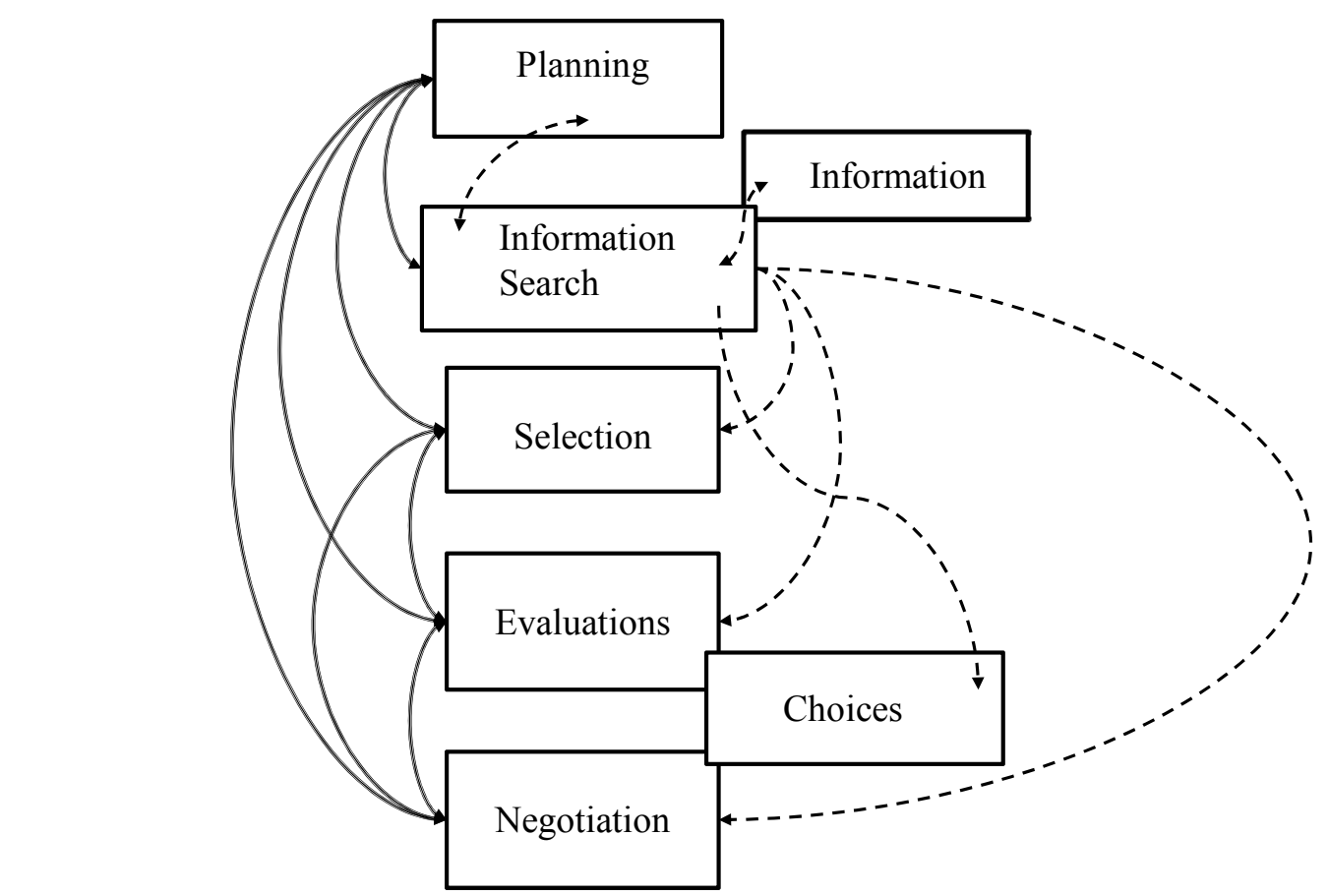

MERPAP (Verville \& Halingten 2003)

- Dotted arrows indicate the flow of information between processes

- Solid arrows indicate recursive nature of activity/feedback/input between processes

Figure 1 - MERPAP Process

While Rao and others suggest methods for system software selection that are linear in nature, the reality of the selection process is that it is highly non-linear.

Verville constrains the selection process with eight conditional statements:

1. MERPAP begins with planning

2. MERPAP ends with negotiations

3. MERPAP is a non-linear process

4. Some process elements are executed concurrently 
5. Some processes are embedded i.e. a process designed to perform one specific function and produce a specific deliverable is a contingent part of another process

6. Apart from the Choice process, all system elements are iterative

7. Apart from the Choice process, all system elements are recursive

8. Each process defines and resolves into deliverables which are used by another process

One research group (Bernroider, E.W.N., Stix, V., 2006) adopted a utility ranking methodology (URM) with the Data Envelopment Analysis (DEA) technique to create an enhanced multi-attribute decision making (MADM) method. URM was applied to a range of attributes, to describe the desired profile of the compatible solution software, and DEA was applied to examine the effect of the selection criteria over a broad range of possible satisfaction options. The decision committee did not use a specific methodology to evaluate the organizational fit to align the new software selection with the company's strategic focus.

Bakas et al., (2007) propose a qualitative holistic methodology to facilitate the selection process for an ERP system, and identify the continuing need in the literature for simple and cost-effective methods and methodologies that do not require, "expert skills, and ...large amounts of time and resources"; and that those that do exist fail to comprehensively cover "strategic fit and functional integration". They point out that 
small companies in particular fail to use the available evaluation techniques for ERP selection (Bernroider, E.W.N., Stix, V., 2006).

Uta, Intorsureanu and Mihalca (2007) suggest a number of possible criteria for the selection of ERP software. They also propose a categorization scheme for the requirements dividing them into the following categories; general requirements, administration and security, reporting, web access and integration, vendor characterization, and costs. They reiterate the finding that there is an intrinsic ambiguity in the classification of requirements and their associated evaluation criteria, but do not attempt to propose a solution to this ambiguity. A methodology approach to classifying the requirements and to developing a clear association between the requirements and the evaluation of system options can help to resolve this situation.

Another quantitative approach to the ERP system selection problem is proposed by the research team Liao, $\mathrm{Li}, \mathrm{Lu}$ (2007). They define the problem as a multi-attribute group decision making problem (MAGDM), and focus on the concept that it is difficult for a single decision maker to consider all aspects of a problem. Accordingly, they try to address the vague and imprecise information available in a decision process by means of fuzzy linguistic variables. They present a model based on the 2-tuple linguistic information processing, proposing a similarity degree algorithm to aggregate the objective information about ERP selection criteria into a common set of linguistic variables. They apply their methodology to a hypothetical linear construct of ERP 
selection, combining external weighting from external contributors and internal weighting inputs from project team evaluators. A more extended application of the decision processes proposed may have value as a component method within a comprehensive solution to the selection process.

A Decision Support System for the selection and evaluation of ERP systems is proposed by Zaitun and Zaini (2008). The team has issued a short paper indicating that they have developed a Web based DSS tool which they have named the selection, implementation and evaluation of an ERP based system (SEEBAS). The paper outlines the framework of the ERP implementation success factors model, which allows online completion of a survey of user defined success factors for a six year period. No further information is provided on the web based ERP selection tool. The authors suggest that ERP success factors information sharing would be enhanced by facilitating a Delphi process for success factors, benefit measurements and productivity indicators.

The Balanced Scorecard (BSC) approach (Kaplan R. , 1992) (1996), which identifies the performance drivers related to strategic objectives that underlie the critical success factors of a project, is adopted by Cebeci (2009) who then applies fuzzy- AHP to this set of variables to select an ERP system for a textile manufacturer.

Shankar and Tiwari (2005) apply the balanced scorecard approach to a computer logistics problem using the Analytic Network Process (ANP) (Saaty, 1999). Leveraging this 
approach, a study of ERP selection using a criteria set of five generalized variables; financial, general, system control and software design, production planning, and data and knowledge properties was undertaken (Yazgan, H.R., Boran,S., Goztepe,K., 2009). The team employed both the ANP method and developed a method to train an Artificial Neural Network (ANN) to synthesize expert judgment of knowledgeable individuals from various organizations. This synthesis is proposed to remove some of the ambiguity of interpretation suggested in earlier work, and to improve the objectivity of the evaluation process. This work does not provide any clear process by which the criteria, which are essential to the final decision, are identified and validated. The process of ERP selection begins with fundamental decision processes at an earlier stage than is proposed in this paper.

Karsak and Ozogul (2009) propose an evaluation scheme that employs Quality Function Deployment (QFD) as the basis for formulating a decision framework for the MCDM of ERP selection. Recognizing the imprecision inherent in some of these concepts, a fuzzy linear regression model is used to determine the target levels for the variables related to the characteristics. The researchers then apply zero-one goal programming to make a selection. This study proposes a method which may have application as a component of an overall methodology, but does not address the entire continuum of decisions to a sufficient degree to represent a complete solution to the problem. 
Bakas et al. provided a strong literature review in their paper (2007), at that time there were only sixteen papers available that dealt with ERP selection processes. Seven of the papers proposed quantitative methods for surveys or evaluation and selection of ERP systems. Of those only five dealt with step-by step process to either evaluate or select an ERP system; our study has identified six more papers since 2007 which deal with ERP selection using either quantitative or qualitative methods. In all cases, the quantitative methods for ERP software selection use the same schema: identify a framework for analysis, derive evaluation criteria from the framework, identify a theoretical comparison algorithm and apply it to the derived evaluation criteria to determine which of a narrow group of candidate systems is best fitted to these evaluation criteria.

The process of selecting an ERP system is a continuum of events and decisions, which culminate in the final evaluation of a set of selection criteria that must generate a ranked set of optional solutions. Almost all of the foregoing research deals with mathematical models, which allow the final selection to take place. The models fail to address the underlying questions of how the process gets to that point of decision. With the demonstrated excessive failure rates of ERP implementation, many of which are attributed to poor selection processes, a methodology which tracks the entire decision process, and provides a set of tools to achieve a good outcome is badly needed.

No single study has addressed a methodology for identifying which ERP systems are the best candidates for evaluation by a SME; this may be due to the narrow group of 
candidate systems available at the top end of the ERP market - SAP, J.D. Powers etc., which have been the focus of many prior studies. For small to mid-size companies, facing the prospect of selecting from more than 200 ERP systems, the choice of candidate system is a non-trivial project. In no case are there any attempts to cross correlate the ERP system client's raw requirements to the functional capability set of the available software systems. Since, many studies have revealed that, the match between the requirements and expectations, and the functional capacity of a software system is a root cause of success or failure in ERP implementations (Bernroider, 2001) (Capaldo, Iandoli, Rippa, Mercanti, \& Troccoli, 2008) (Chang \& et al., 2008) (Ferman, 1999), a methodology to perform such a comparison is needed. An extensive literature review reveals that no methodology exists to provide a generalized, proceduralized way for a SME to select the right ERP System from the many systems available in the marketplace.

It should be noted that industrial practice, with respect to ERP system selection, is driven by systems employed by consultation entities which have affiliations with particular software providers, as representatives, so do not have an objective basis for best value estimations from a broad base of technologies. Those selection systems that do exist are largely proprietary in nature and are limited in the information available. There is little or no cost information available from these private resources, and users are invariably cautioned that cost information is highly volatile and generally unreliable within these resources. 


\subsection{ERP Implementation}

Even in small businesses, there has been an increasing realization that in order to remain competitive, the need for technology integration is increasing. Until recently, the implementation of ERP systems has been mostly the domain of large businesses, requiring significant investments of resources (Andriole, 2006). The scale of the investment for a large business enterprise for an ERP system is frequently several million dollars, and extensive use of consultants and internal staff are required for implementation. While a small business is not required to invest at these levels, the proportionate resource commitment is still high (Davenport T., 1998).

Two issues are driving large portions of the small business community to adopt ERP;

firstly, as the natural supplier base to large businesses, there is a strong impetus for adopting ERP systems that can integrate at a higher level with the client's systems, thereby making the small business a better fit in the supply chain; and secondly, ERP providers are developing new offerings that provide much of the functional capability at lower cost than those of the major systems, such as SAP \& etc. (Mabert, V. A., Soni, A., \& Venkataramanan, M. A., 2001) (Chen, 2001). There are still challenges though, in particular the expenses of implementation and reengineering of business processes to accommodate these more sophisticated systems, and a poor internal facility to gauge the value of an ERP to the bottom line (Nah, 2001). More recent research has explored the total cost of ownership which includes acquisition, implementation, maintenance, training 
etc. (Babey, 2006). Of course, access to information about these costs is frequently regarded as proprietary to the ERP vendor and is difficult for a small business to obtain. In their review of the developments and directions of operations management (Jacobs \& Bendoly, 2003) suggested that the domain of research interest in ERP systems has bifurcated into two main paths; one which focuses independently on corporate capabilities with respect to ERP strategies, and those which deal with the respective benefits and costs of IT systems. The proposed research will address the interrelationship of both of these factors as they apply to the selection decision process and investigate the effect of success criteria of implementations at small manufacturing businesses. Several researchers have demonstrated that it is an accepted fact that business systems are more appropriately modified to accept existing software implementations of ERP systems than vice versa (Al-Mudimigh, Zairi, \& Al-Mashari, 2001).

Due to the paucity of empirical research, case studies are proposed to represent a strong foundational contribution to the domain of academic research into issues related to success of ERP implementation projects (Madapusi \& Ortiz, 2009) (Wei C. , 2008) (Motwani J. , 2005) (Kumar, Maheshwari, \& Kumar, 2002) accordingly, this study will address the experiences of small businesses engaged in defense manufacturing.

In a review of the research agenda of ERP studies (Al-Mashari M., 2003) a contextual framework is proposed which includes taxonomy of ERP research. The outcome of the proposed research will address several elements of the current ERP research agenda and extend both the academic and practical uses of the fit assessment process. 
Chang (2008) proposed that social factors, perceived consequences, complexity and other elements, as measured by such models as the Triandis framework, drawn from Triandis' “Theory of Interpersonal Behavior" (Triandis H. C., 1971) (Triandis H. C., 1980), can affect the user's perspective when adopting new technology. Chang's study indicates that the actual success vs. perceived success of an ERP implementation is a very real facet of the user's overall view of the system and its value to the enterprise. They conclude that active and positive managerial support, with a socialized and reasoned approach to establishing a "supportive social environment" is critical to both actual and perceived success.

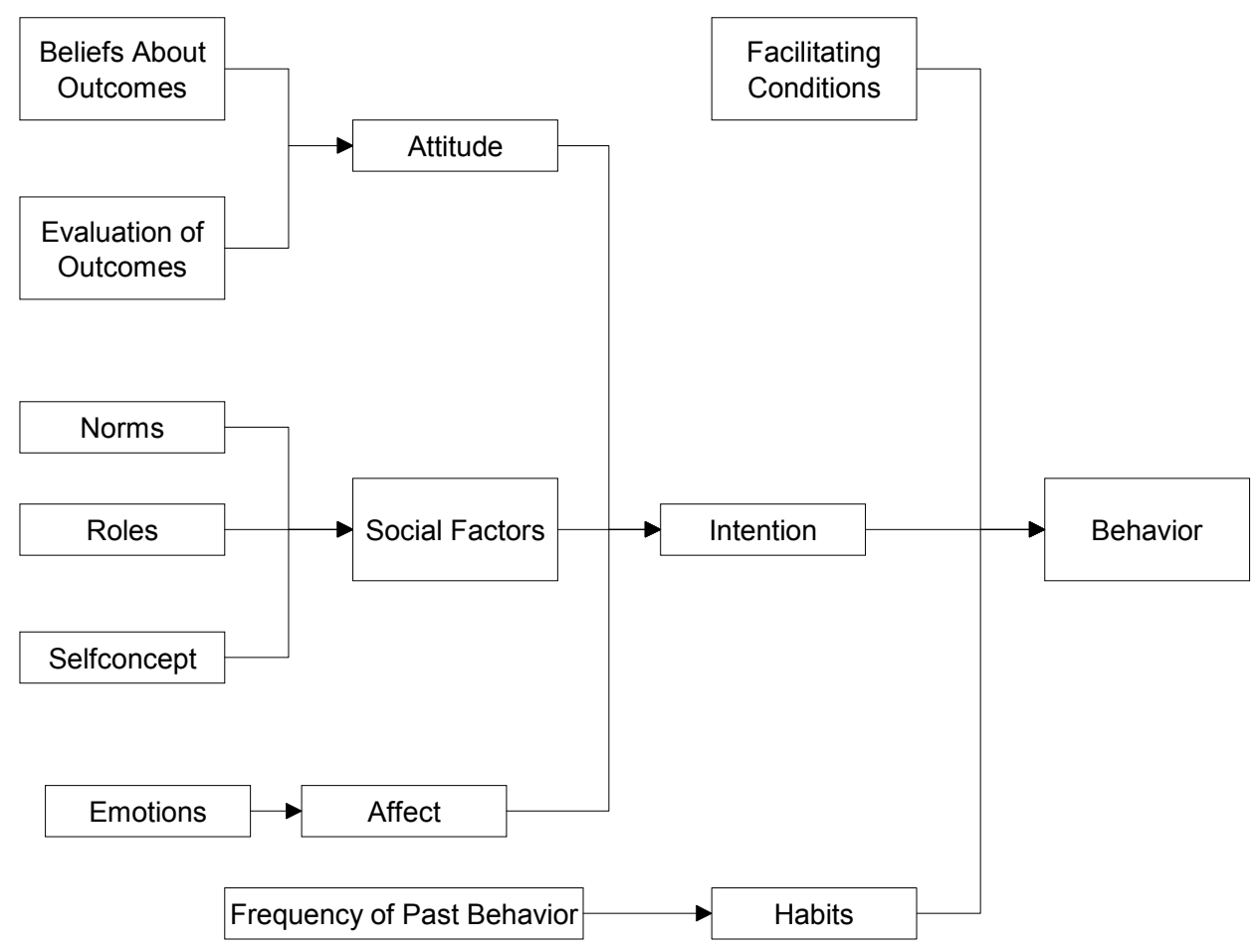

Figure 2 - Triandis' Framework 
(Wang \& et al., 2007) have proposed a fundamental relationship to ERP implementation success, based on three parameters; Client "absorptive capacity", defined as the ability of a team to recognize value, assimilate and utilize new, external knowledge; the competence of the implementing consultant; and the effectiveness of the knowledge transfer occurring during the implementation phase. More recently, (Morton \& $\mathrm{Hu}$, 2008) have suggested a review framework based on structural contingency theory and drawing on the use of Mintzberg's seminal work on organizational structure (Mintzberg, 1979). ERP systems are perceived as assets within a company when the social acceptance factors are positive with respect to the benefits of an improved work environment are recognized. Such social acceptance is enhanced by positive support from managers, and demonstrated benefits such as compatibility of the ERP system with an individual's working mode, and early demonstration of these benefits (Chang \& et al., 2008).

Several studies have demonstrated support for the view that a gap between the ERP systems processes and those of the business are inevitable (Gefen, 2002) (Sawyer, 2000). There is an unresolved discussion regarding the options of undertaking business process reengineering that accommodates the ERP systems underlying processes, or whether to customize the software to accommodate existing business practices. Hong and Kim (2002) assert that the ERP fit and operational contingencies are both important facets in establishing success of implementation, while also indicating that the decision to change the business process to accommodate the ERP process is sometimes preferable to an 
extended effort to change the ERP model to fit the business. Boudreau and Robey (1999) adopt the view that organizations need to be changed to fit the ERP software. Other studies conclude that the adaptation of the ERP system to the businesses operational processes is preferable (Markus, M.L., \& Tanis, C., 2000), (Holland, C.P., \& Light, B., 1999). A third opinion seems to forming around the philosophy that ERP systems should be modified to fit existing or reengineered business processes (Brehm, Heinzl, \& Markus, 2001) (Davenport T., 1998) (Glass, 1999). Gyampah and Salam (2004) explored the possible effects of the Technology Acceptance Model, and its implications on the perceived and actual success of ERP implementation. They also explored the effects of training, and communications in managing expectations and advancing the acceptance of new technology in the workplace (Gyampah \& Salam, 2004).

A case study of an implementation failure is used to elucidate the key skills and integration strategies essential for implementation success. The study identified five core competencies: 1. Adopting a change strategy development and deployment, 2. Employing enterprise-wide project management, 3. Using change management techniques and tools, 4. BPR Integration with IT, and 5. An understanding of the strategic, architectural and technical aspects of the software installation (refers specifically to SAP R/3) (Al-Mashari \& Zairi, 2000).

In a later work, the team proposes an integrative framework to aid in ERP software implementation, and notes several additional deficiencies that must be overcome to succeed in implementation. These include a recommendation for an evaluation process to monitor short and long term benefits from ERP usage, the inclusion of an assessment 
of IT competencies, and the essential nature of balancing strategic objectives in the ERP selection process. While this paper does propose an integrative framework for the implementation phase, it fails to extend the domain of analysis to the selection process itself, although it does recognize its importance to the overall success of an ERP introduction (Al-Mudimigh, Zairi, \& Al-Mashari, 2001).

Motwani et al. (2005) suggest that their case studies reveal a complementary result, specifically noting the positive impacts of a cautious, evolutionary, bureaucratic implementation process, backed with careful change management, network relationships, and cultural readiness. 


\section{RESEARCH PLAN}

In this section the research outline is presented to identify the main procedural and process steps that were undertaken in the research effort. We begin with an outline identifying in a general sense the themes and techniques used to address the topics within the study. We then detail the specific methods of the research, data collection and analysis that were employed.

\subsection{Overall Approach}

The overall approach to this research has been to employ a meta-methodology to develop a new methodology to select an ERP system for a SME; we test underlying assumptions about the desired outcome of the new methodology, and inform the methodology development by means of a survey; and, we test the methodology by means of a case study, and expert verification.

Theoretically, the outcome of the analysis of the triangulated results of each of these approaches may serve to show; convergence, a broad agreement of the results of the approaches; inconsistency, which may suggest alternative solution mechanisms to the chosen approach; or, contradiction, indicating an invalidity or weakness in the approach.

Thomann's meta-methodology is utilized as the development armature for the new methodology. Thomann's meta-methodology provides several advantages; it provides a reliable and proven development path for the methodology, as opposed to ad hoc and 
tailored subjective methods more commonly employed; the meta-methodology is a structured approach, and allows the incremental buildup and progressive testing and revision of the methods that constitute the methodology; Thomann's meta-methodology, in particular, allows the methodologist discretion in selecting the methods and techniques that are most applicable to the objectives; third party review, validation, and commentary are intrinsic elements of the meta-methodology; allowing early validation and a high degree of confidence in the final methodology.

A methodology is designed to select an ERP system for an SME again applying Thomann's meta-methodology; including, a survey assessment of SME enterprises, which informs methodology development regarding the desirability and practicality of the new methodology, and the insufficiency of the existing selection processes. The survey also contributes to the procedural design of the methodology (Thomann's step VI) by answering the questions regarding the weighting of attributes.

The methodology is tested by performing a case study, the practical outcomes of the methodology can be analyzed to test specific functional behaviors that are or should be present in the selection process, and provide empirical evidence for the practicality and operationalizability of the more generally applicable methodology. The methodology will be reviewed by practitioners and other experts to determine the validity of the methodology. 
Finally, we provide a summary of the research results and propose some new paths for future research.

\subsection{Detailed Research Plan}

Thomann's meta-methodology has at its heart three objectives; determining the purpose, development of the steps that constitute the new methodology, and testing the methodology. The detailed research plan follows the meta-methodology, and identifies the required activities, methods, procedures and tools to accomplish them.

The following seven steps, with expansions to include specific methods (see Appendix A), are proposed by Thomann, as sufficient to address the development of a new methodology:

I. Put the methodologist in touch with the problem.

II. State the purpose of analyzing the problem area and determining a purpose that will solve it.

III. Test the purpose
a. Is purpose desirable?
b. Are existing methodologies insufficient?
c. Is it practicable?
d. Is it operationalizable?

IV. Analyze the implications of the purpose for the development of the methodology.

V. Operationalize the purpose. 
VI. Design the Procedures (Using Thomann's meta-methodology as an armature)

VII. Test, and revise the purpose or procedures if needed.

Task 1: By applying Thomann's meta-methodology one can determine the purpose, and develop all the necessary steps that are required by the methodology; the methodology is then tested to ensure it accomplishes the purpose.

An important facet of the Thomann meta-methodology is the flexibility that is deferred to the methodology developer with regard to the order of the steps; the elements that are most clearly left to the discretion of the developer are sections V, VI and VII, with the strong suggestion that sections VI and VII can be addressed simultaneously i.e. Development and testing can be simultaneous activities, allowing recursive and iterative processes. (See Appendix A)

Task 2: The analysis of the problem of selecting an ERP system for an SME will be decomposed into manageable parts by applying a process modeling approach conforming to the Integration Definition for Function Modeling (IDEF0) developed by the USAF Systems Command. The objective of the NIST standard 183 is to provide a means to completely and consistently model; activities, actions, processes, and operations that are required by a system or enterprise. In addition the modeling technique shows the functional relationships and the data that support the functions. 


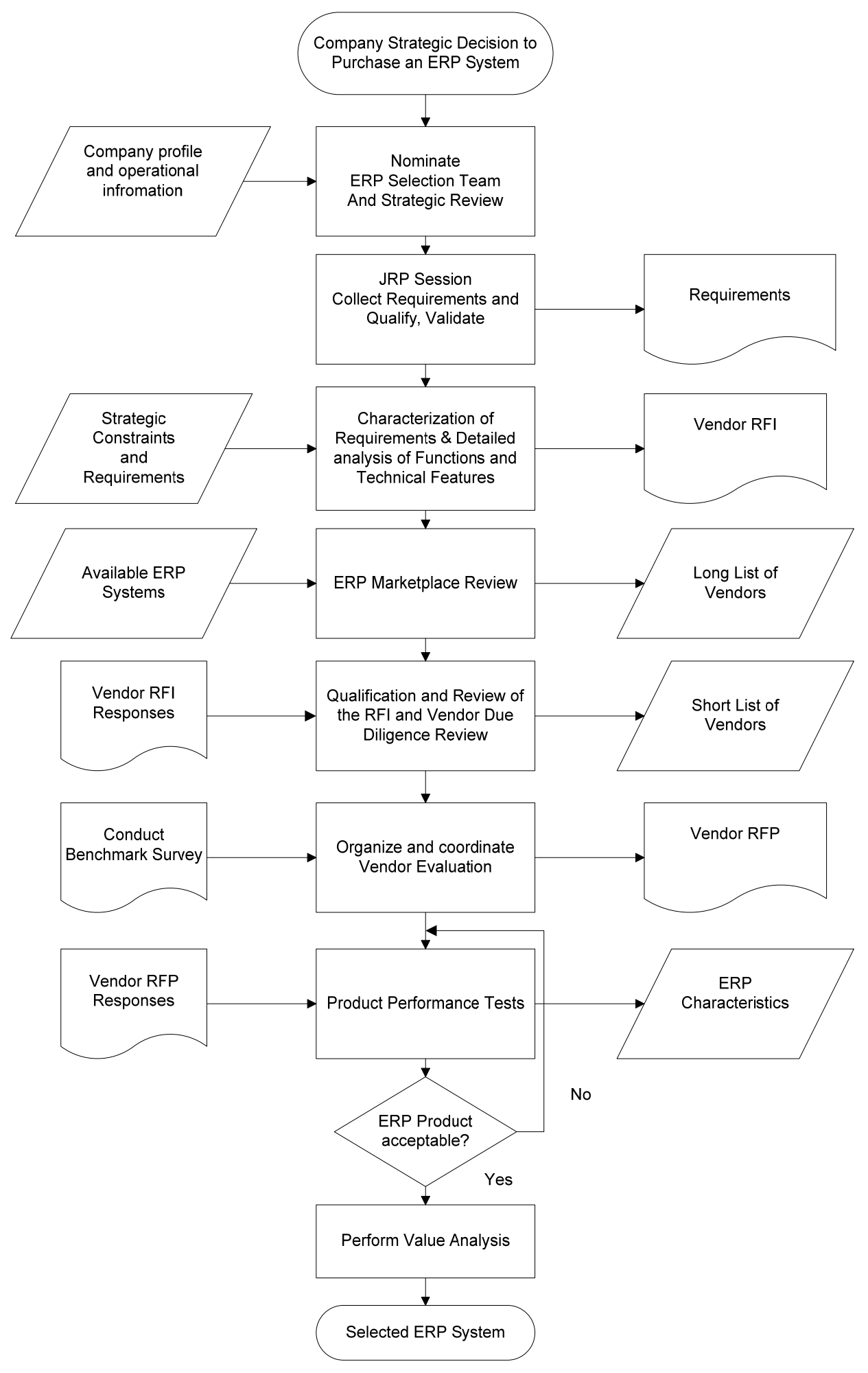

Figure 3 - Flow Chart of ERP Selection Methodology 
The decomposed structure of the methodology elements is used to analyze and identify the individual tasks and methods which will fully operationalize the methodology. We will evaluate and select a suite of systems engineering tools which allow the execution of the component activities and provide the necessary data for formulating knowledge and information for succeeding stages of the methodology.

\section{Control/Constraint}

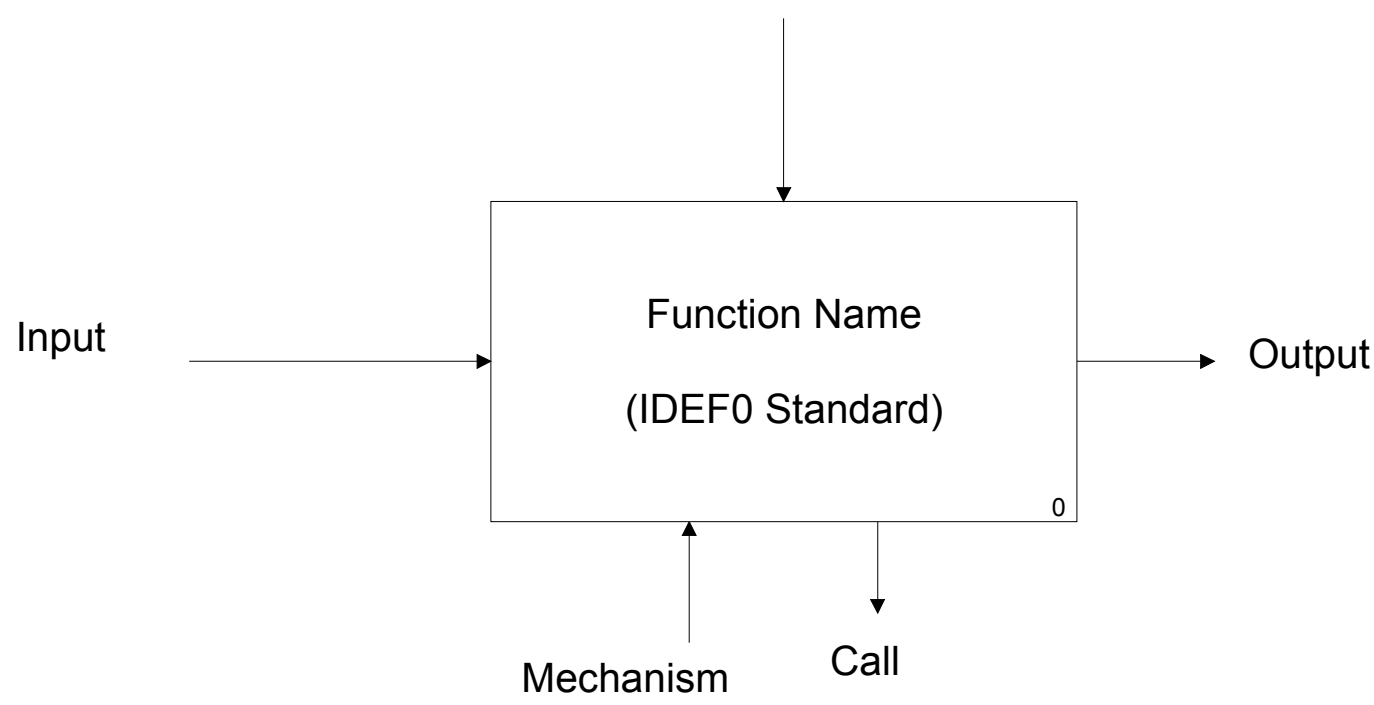

IDEF0 Standard Arrow Positions and Roles. NIST Std. 183

Figure 4 - IDEF ICOM Description

A survey is conducted to determine benchmarks and industry best practices from a group of SME's that have successfully selected and implemented an ERP system within a five year period. A multi-respondent survey is mailed to qualified candidate organizations which contained sixty eight questions designed to test nine hypotheses. 
In order to have a high degree of compatibility of the survey results to the targeted business sector, the survey was directed to a group of small manufacturing businesses, with successful recent experiences in the selection and implementation of ERP systems. Other qualifying factors were:

- Annual Sales Volume (less than $\$ 100 \mathrm{~m}$ )

- Types of Products (components, sub-assemblies, design-build products etc)

- Number of employees (less than 500)

- Years in business (less than 30)

Twenty three companies were invited to participate in the survey, fourteen companies agreed to provide answers. Of the survey responses received one was found to be inconsistently completed, providing a response rate of $(56.5 \%)$ which is acceptable and exceeds the rate of response of other studies in this area (Paulraj, 2005) 23.2\%; (Krause, D.R., Pagell,M., \& Curkovic,S., 2001) 19.6\%.

The survey was designed for a response time of one hour or less, since we requested high level executive and senior manager level responses. We provided an assurance of anonymity and confidentiality of the respondents and their company information to encourage frank and open responses.

The research survey is directed at improving the understanding of the interrelationship of the selection process with the IT infrastructure, business processes and implementation of new ERP systems. The underlying data will be analyzed with reference to the testing and validation of the selection methodology. Accordingly survey questions were developed 
which address; change strategy, executive facilitation, project management competency, IT based competency, staffing, training, BPR competency, software selection issues, and project communication and support issues. The data from the survey will inform the testing and revision of the methods employed in the selection methodology; to ensure priorities are correctly valued with respect to industry norms, and that the breadth of analysis in those methods are sufficiently general to make the methodology reliable for a wide range of small and medium-sized businesses. The survey was designed to test implications and design of the new methodology by seeking input on the following theoretical propositions:

Even a successful implementation of a newest of strategic changes, as produced by an ERP introduction, cannot ensure business success. The comprehensive use of centralized data does not intrinsically improve efficiency, and is only when the system has the ability to provide clear, unambiguous reporting with regard to measured performance objectives that the link between business strategy and the change strategy can be evaluated. It is essential that the strategic goals are in place, and justify the investment, before the organization implements an ERP system (Motwani J., 2005).

It is clear that most executives have a clear belief that the introduction of an ERP will provide business benefits from the outset, and that executive's apply pressure to achieve the final state where the ERP is operating seamlessly and is facilitating such benefits. One of the most frequently identified success factors for ERP implementation has been the ability to focus executive attention on a bigger picture, rather than the technical 
elements of the project (Davenport T. , 1998) (Gupta, 2000) (Rao, 2000) (Vaughan, 2001) (Wu, 2008). Recognizing that ERP commitments are long term change processes, rather discrete events, is a key to ensuring that resource commitments are adequate and enduring.

Project management with respect to ERP implementation conforms to most principles of good practice. It is essential to perform a strong initiation process, ensuring that the scope of the project, resources available and timeline expectations are set early and that a chartering process is performed to ensure that lines of reporting and project objectives are fully known to all parties. It is accepted that the unexpected will occur, however good project discipline, appropriate change order mechanisms and open communication can help prevent ‘scope creep’ (Trepper, 1999) (Kumar V. M., 2003).

ERP implementation can affect either or both the hardware and software environments at a company. The degree to which the company is impacted and whether those impacts are positive or negative depends in a great part on the employee capability as much as the technical capacity of the system. Issues related to the management of the change process from the IT hardware and system perspectives have been long noted as critical causal factors in the failure of ERP implementations (Evangelista, 1998) (Hill, 1997). Staffing

An ERP system introduction changes many performance aspects of an employee's position, and the extent to which managers can identify and address personal, 
departmental and organizational issues related to these changes often make for success or failure of an ERP system implementation. Small companies, in particular have many resource challenges in addressing these aspects of change (Ferman, 1999). For a small company, the introduction of an ERP system may require a vastly higher level of technical competence and sophistication, in many skill areas, than has previously been required from the employees (Hill, 1997). The extent to which changes to assignments, altered lines of reporting and new decision processes can be accommodated by; training, hiring or reassignment, are critical to ERP success.

Almost everyone in a company must have some level of knowledge about ERP systems in order for it to be successfully implemented. The knowledge required can be introduced through both formal and informal training, and is usually divided into two levels; those requiring a broad and conceptual knowledge of the system, and those whose everyday duties require a sophisticated and in-depth knowledge of the system, its functionality and how it is interrelated to all enterprise functions. Some level of training can be provided through in-house training by managers, while other technical proficiency related training must come from vendors or consultants (Al-Mashari \& Zairi, 2000) (Davenport T. , 2000) (Muscatello, 2002).

ERP systems are intrinsically change agents for a company, and there are various strategies that must be decided upon before the implementation begins. A company in this position has three options; firstly to adapt its existing practices to the underlying business philosophy of the ERP system. In this scenario the routines, and systematic 
processes that are contained in the ERP logic become the objective for the company's overall business processes, in other words the company becomes systems friendly towards the ERP. Alternatively, the second option is to try to customize the ERP system to the company's existing processes, force fitting the existing business systems into the structures that are employed in the ERP system. Most implementation strategies try to find a middle ground where an ERP system is chosen to find the greatest degree of accommodation of the existing business processes and to customize the ERP where there is a lack of functional capacity in the system to accept existing processes. A third alternative is to engage in simultaneous ERP implementation and BRP process improvement; studies suggest this option to be a highly risky approach and counsel against the practice. Both ERP implementation and BRP are highly sophisticated and resource intensive projects, finding a modest balance is crucial to success. Although some research suggests that fully $35 \%$ of the dollars expended in the reengineering of a company deploying an ERP system are attributable to BPR, there is a strong relationship between the careful management of BPR investments and ERP success (Muscatello, J.R., Small, M. H., \& Chen, I.J.,, 2003) (Motwani J. M., 2002) (Millman, 2004) (Olson, D.L., Chae, B., \& Sheu, C., 2005).

ERP software system selection and the effects of appropriate matching of the chosen system to the organizational requirements and objectives is one of the least studied areas of research (Verville, 2003), yet it has been asserted that firms that analyze the software "fit" and apply them to software selection increase the likelihood of successful implementation significantly (Yusuf, Y., Gunasekaran, A., \& Althorpe, M.S., 2004). The 
selection and implementation of an ERP system is a highly specialized process, fraught with obstacles and pitfalls to a novice. In small businesses in particular, the likelihood of having the right kind of expertise on staff is very unlikely; even large companies have similar deficits. Even when such expertise is resident in the company, the day to day operational needs of the employee's full time assignments make them a unavailable for the assignment. It is proposed in several studies that management should employ knowledgeable consultants to organize and assist in the selection assessment phase (Chen, 2001) (Davenport T., 2000).

The lack of socialization and acceptance of the change process and the alterations in information flow within an organization can be serious causes of 'revolution resistance', and they are obstacles to change. The positive effect of good communication planning and the advantages of effective communication processes are observed by several research efforts (Motwani J. M., 2002) (Muscatello, J.R., Small, M. H., \& Chen, I.J., 2003) (Chang \& et al., 2008). Accordingly, the strong application of project management protocols has demonstrated significant benefits to previous implementation activities. In today's work environment, where employees are expected to take equity in the results of their actions, clear communication of the need for change and the advantages that the change will exhibit to their workload is essential (Olson, D.L., Chae,B., \& Sheu, C., 2005). The degree to which project level information is disseminated has a key effect on the perception and enthusiasm exhibited by all members of the organization. Good communication can help dispel fear and anxiety associated with the change process. 
A focused survey of small businesses was employed to test the theoretical constructs proposed above, with the intent to gather information regarding ERP selection and implementation success and failure criteria. In addition a survey element was included to discern the past experiences of successful ERP implementations into the development of a more comprehensive system selection process. The test of these constructs by the survey conforms to the overall procedure suggested in the literature; however as a focused study it addresses solely the attitudes and opinions of the target group (Luo \& Strong, 2004) (Muscatello, J. R., Small, M.H., \& Chen, I.J., 2003).

The literature review suggested that such constructs have been employed by others for broader studies, but that the differentiation of the experiences of small businesses has only just begun to be studied. The survey questions conform to earlier studies, and were developed using principal component analysis, in which all observed variance is analyzed. The technique was used to define the questions by combining variables under test into a useful survey. In order to correlate and analyze the data from this study with previous research, the survey scoring system was constrained to a Likert-type scale (Likert, 1932) with adjectival ratings ranging from a low value of Very Strongly Disagree to high values of Very Strongly Agree. Corresponding numerical evaluation of each adjectival choice ranged from 1 to 7 .

Task 3: The methodology of the case study followed a four step process; design of the case study, conduct of the case study, analysis of the case study evidence, and the 
development of the conclusions, recommendations and implications of the information retrieved from the process.

An ERP selection for a small company was conducted, and the experience of the case study was used as a test for the methodology. ABC is an SME dedicated to custom engineering manufacturing of defense related systems. It is a low volume/high mix manufacturer, performing design, development, and system integration for custom engineered systems. In addition to its manufacturing capability, the company has programs which perform Research and Development (R\&D) as a client service, and a small engineering resource capability which is used for Internal R\&D (IRAD), and competitive research work such as SBIR/STTR from client agencies.

The company had made a strategic decision to adopt an ERP system, and by acting as a consulting entity we were provided an opportunity to test the ERP system selection methodology developed in earlier work. Notably, the company had attempted to employ an ERP system several years earlier, but had had a very unsuccessful experience because the system had been chosen by a sponsoring large business which had very little concept of the unique operational characteristics of a small enterprise. The committed participation of its own staff was a very important factor for this company in choosing a self-selection process.

The case study experience is used as a testing and development environment to implement the methodology, and to identify the best value ERP system from the systems 
under review. As a testing environment, the case study will be analyzed to determine if we have identified the best techniques, or methods within our methodology, and to address any weaknesses found in the process, or if the example suggests any alternatives to the methods employed.

The case study represents a component of a triangulated research strategy; this particular research effort is characterized as a methodological triangulation, since its data are relevant to the development efforts of the final methodology, along with the results from the survey which inform both the weighting processes within the methodology and points to larger issues of the methodology development and the best practices that should be incorporated.

The case study is completed with a review of the requirements, using principal component analysis (PCA), in order to test the methodology's underlying assumption; that the requirements represent a sufficient description of the company's processes to allow evaluation of the ERP systems. PCA also allows us to determine whether the number of factors, variables, can be reduced in the analysis and evaluation, without incurring greater risk of a poor outcome.

Task 4: The methodology was reviewed by practitioners and other experts to determine the validity of the methodology. Third party independent experts in the fields of ERP design, consulting and implementation will be surveyed to evaluate and validate the methodology. The experts were provided with the methodology, and asked to evaluate 
each major phase and determine if the methodology meets its primary purpose. The responses were analyzed to check whether the methodology is viable, and practical. 


\section{RESULTS}

\subsection{Methodology Development}

The process of methodology development begins by engaging Thomann's expanded meta-methodology and laying the groundwork for the understanding of the problem.

I. Put the methodologist in touch with the problem.

Thomann suggests that an understanding of the entire methodology development process has a critical and useful purpose, and allows the methodologist to navigate the formulation of a comprehensive and accurate response to problems at hand. He suggests a simple method which we elected to use, namely; the interest of the methodologist. Our objective is clearly to develop a methodology to select an ERP system for a small or medium-sized business. We have a clear problem because based on the literature review no methodology for ERP selection for SME's currently exists.

As an alternative, a more complex method is offered as a systematic approach to problem analysis; Coffing's Client Demand methodology however this approach is not applicable to this problem.

II. Purpose of the methodology - a methodology to select an ERP System for an SME.

As a crucial next step, Thomann charges us with the responsibility to investigate the problem area, with the purposes of; placing our responses and understanding in line with 
previous research, ensuring that the methodologist is fully apprised of current solution approaches, and that our response is within the logical confines of practicality. Thomann's approach requires that we review the literature; based on an extensive review, See Chapter II, it is clear that no methodology exists which allows the selection of an ERP system by a small or medium-sized business. He also requires that we participate in the community of interests for our study area, by initiating and maintaining communication with other practitioners and users. The case study participants and survey respondents were a primary and valuable source for discussions regarding appropriate methodological approaches, identification of processes, procedures, methods and reviews of proposed methodology steps for the selection process. These discussions also informed the development of the formal methodology presented in this work. In addition, we validated the new methodology by reference to a committee of experts, drawn from commercial practice backgrounds.

Previous ERP selection and implementation processes over an eight year period were reviewed to identify technical process, procedural and methodological similarities and differences to inform this methodology development process. In addition, the literature review and discussions with ERP consultants and implementers were used to inform this development activity. Where possible, we reviewed commercial evaluation and selection methods, although access to these resources is severely limited due to the proprietary nature of the systems employed. The objective value of commercial evaluation systems is not clear, since most, if not all, have commercial affiliations and vested interests in 
promoting one or more of the ERP systems that are presented as options in the evaluation tool.

ERP selection is employed classes of client organization; large multi-national businesses and SME's. The vast bulk of the selection approaches in the literature deal with the selection of an ERP system for a large business, there are no systematic or methodological approaches for SME's. The constraints and decision variables for large businesses are intrinsically different from those of SME's and are not applicable to the SME ERP selection process. In industrial practice, the selection process is largely ad hoc on the part of the SME or is controlled by vendor representation interests, or through proprietary systems. None of these systems adequately addresses the needs of SME's and a new methodology is needed.

\section{Test the purpose of the methodology}

As identified earlier, the global budget for ERP implementation is projected to exceed \$36 Billion in the next decade. The worldwide market for enterprise resource planning (ERP) systems grew at a 4.8 compound annual rate, rising from $\$ 16.7$ billion in 2005 to more than \$21 billion in 2010, according to a study from the ARC Advisory Group Inc., Dedham, Mass. Since, small and medium-sized businesses produce over $70 \%$ of the value added in the economy (US Bureau of Labor Statistics , 2010) and significant numbers of them are entering the ERP marketplace, the call for a selection methodology tailored to the needs of SME's is highly desirable. 
The professionals and consultants who were engaged in the case study project, and users who responded to the survey, and users who participated in the case study, all reviewed the methods and methodology employed and concurred that a methodology to permit the selection of a best value ERP system for SME's was a desirable purpose.

In light of empirical and anecdotal success in selecting and implementing ERP systems there is clearly a viable way to select a best value ERP system for a SME; this conclusion is supported by the fact that our survey was focused on a subset of small and mediumsized businesses who had satisfactorily selected and implemented ERP systems. The respondents to the survey all agreed to utilize a scaled approach to demonstrate their levels of satisfaction; indicating that the underlying variables are measureable factors. Using Thomann's guidelines to the meta-methodology it is not necessary to demonstrate the operationalization at this stage; it is sufficient to have assurance that the purpose can be operationalized.

The development of a methodology is a desirable and practical approach to select a best value ERP system. Many small and medium-sized businesses have identified and successfully deployed ERP systems, indicating that there is a practical method to identify and successfully implement ERP systems. It is important to note that, anecdotal success does not necessarily assure that the methods used in selecting the system can be reliably incorporated into a methodology. By using systems engineering tools such as IDEF mapping to decompose and map the system, a practical methodology can be designed. 
Considering that, the industry norm for ERP system selections are either highly distorted by commercial representation bias and business influences, or are extremely ad hoc approaches, a methodology employing an unbiased set of techniques and methods is a highly practical approach to achieving the purpose.

As indicated in the literature review, no methodology for the selection of an ERP system for a SME currently exists. The system proposed is therefore unique in its purpose and design. Using Thomann's meta-methodology; meeting this test and having affirmative responses to all the foregoing step III questions, we fulfill all the necessary and sufficient conditions to proceed to the next step.

\section{Implications of the Purpose}

In conformance with the meta-methodology we begin outlining the implications of the new methodology by assigning the first and last steps to the new methodology; these are; first, putting the user in touch with the problem, and last, testing, and revising the purpose and/or the methodology where the test results reveal needed amendments. Since, we have bounded our study area to address only those aspects of the ERP adoption process which deal with the selection of a best value ERP system; the first step is accomplished by briefly reviewing and providing informational briefings to the users who are to utilize the selection methodology. The primary conditions and resource allocations for the overall project of ERP implementation are strategic decisions, and this element of the methodology is accomplished by a review of the company strategy and the strategic 
constraints; budget, personnel resources, and IT Infrastructure, that are available for the ERP system implementation process.

Conforming with Thomann's collaborator Hutchinson's admonition, that each problem implies its own solution and by following the meta-methodology process steps we identify that the process of ERP system selection, using a best value approach, is divided into four major process phases; collecting and understanding requirements and constraints, and establishing a global comprehension of the business and its strengths, weaknesses, opportunities and threats; identifying and qualifying the available ERP systems and their vendors; comparing and analyzing the information gathered in the first phase to the capabilities available in the available ERP systems; and, lastly evaluating which ERP system is the best value option in light of the conditions identified.

The process of selecting an ERP software is divided into five phases; a review of the company strategy; a definition phase in which the strategic and operational requirements are specified; a marketplace analysis, to determine what systems are available, and in which preliminary sorting is undertaken to restrict the later detailed analysis to a short-list of viable options; a comparison phase, in which a more detailed analysis and review is undertaken to focus on the most appropriate systems; and a final selection process in which the primary constraints are considered and a match is found for the requirements that are core to the company's objectives. 


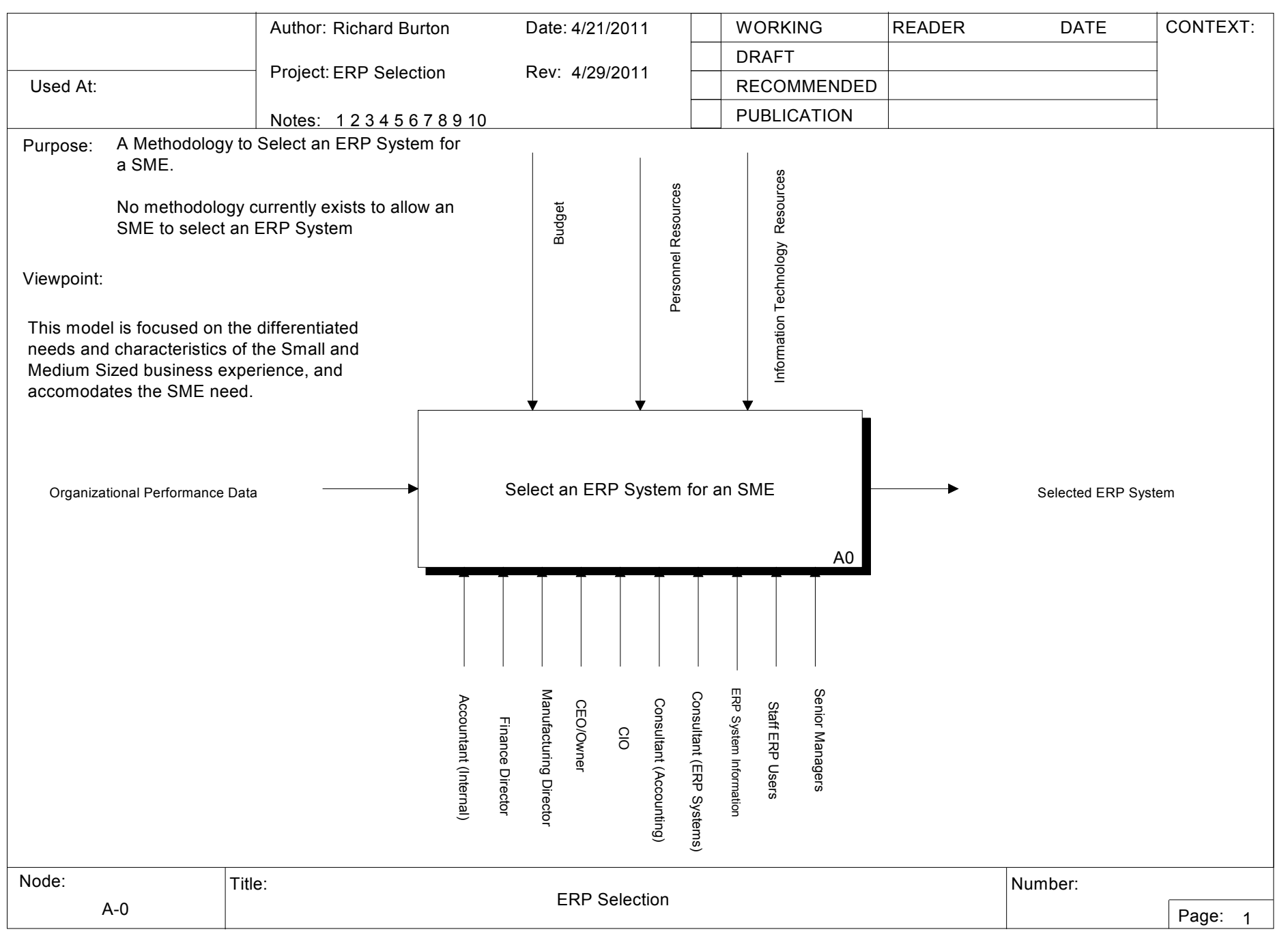

Figure 5 - IDEF0 A0 Diagram 
These phases are further decomposed into activities and functions which are served by methods and techniques drawn from systems engineering approaches; these approaches are either internal processes or are external, relying on vendor and consultant interactions. By specifying the methods and techniques or procedures to be adopted, and applying a meta-methodology approach to designing the entire process, we construct a unique methodology which will serve the designated purpose.

A key initial function is to determine what skills are resident, and available to participate in the selection process; these skills are most economically sourced from internal company expertise, and supplemented where necessary with external expert skills from consulting resources. A characteristic of small and medium-sized businesses is their cost conscious utilization of resources, but it is assumed that consultants are essential for most SME's to ensure that they are fully informed about what has become a complex and challenging marketplace for ERP software systems. The most essential element of this stage of the selection process is forming a cohesive and effective team environment, in which the objectives of the company are fully understood by all parties, and the distinct roles, responsibilities, authorities and expertise of each member is clearly articulated and communicated. 


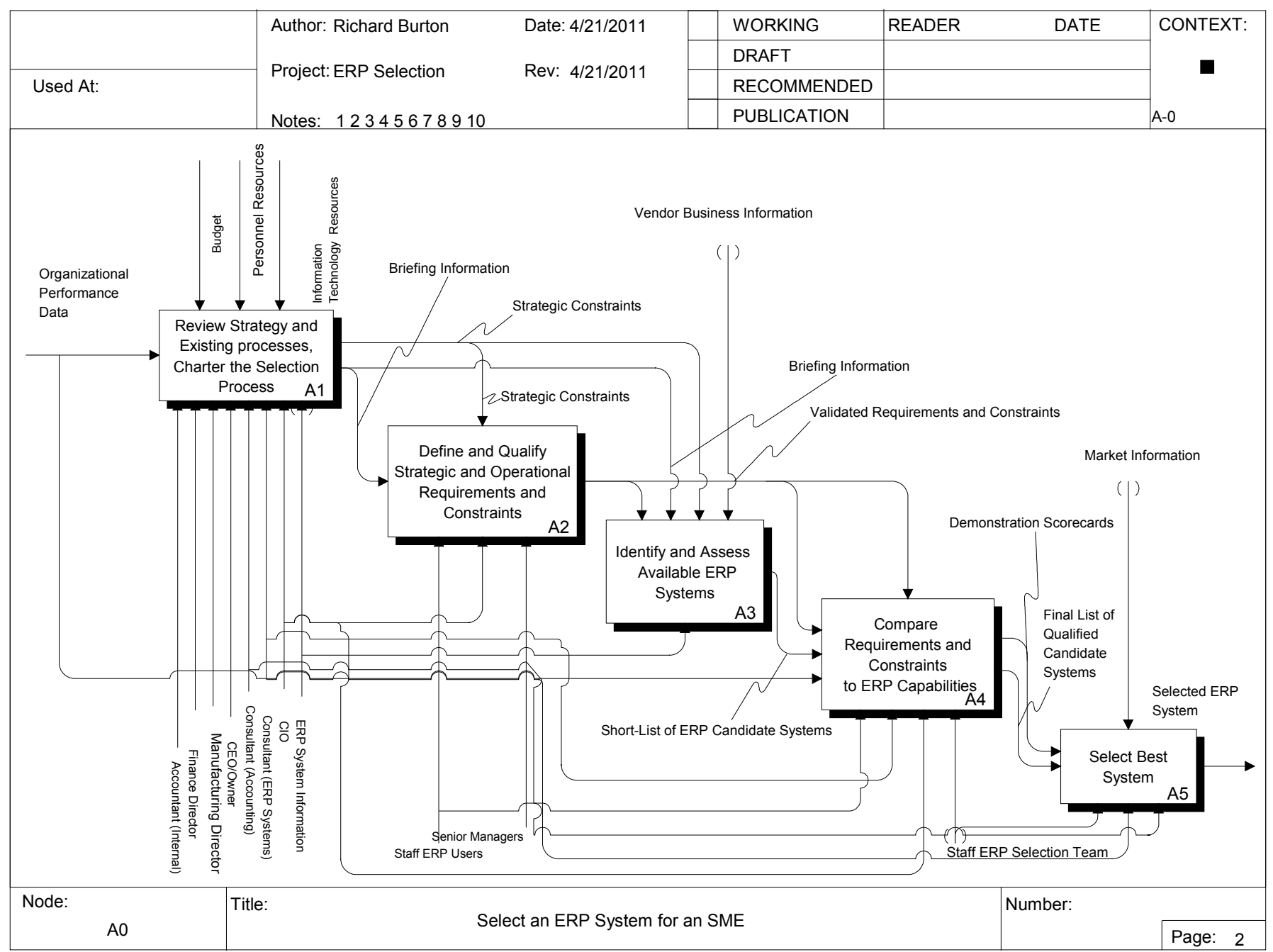

Figure 6 - IDEF0 A0 Select an ERP System for an SME 
Firstly, we review company strategy and operations to garner as complete an understanding of the reasons why the company decided to employ an ERP system, what its expectations are at the strategic level i.e. what outcomes are expected from the ERP investment- fulfilling Thomann's charge, to put the user in touch with the purpose. This process ensures that the methodology users are fully informed with regard to the strategic intent and objectives of the methodology. Since, the methodology is related solely to the selection process for an ERP system, certain decision processes, taken outside the scope of the selection; such as why the company is buying an off the shelf system, have significance to developing a well informed choice. Equally, the availability of staff, for the selection process, budget constraints etc. are all relevant to the context in which the most suitable ERP system is identified.

The strategic briefing must also define which value proposition is most important to the business; the first proposition identifies which ERP system is the alternative (within a defined budget, and exceeding the minimum functional requirement matching level) that has the least total cost of ownership while gaining as much functionality as possible. i.e. the company will choose the lowest price option, with the understanding that if two or more systems are available at the same price point, they will chose the system with the greater functionality. The alternative logic to identify a best value option is that the company wants the maximum level of functionality available, (within a defined budget, and exceeding the minimum functional requirement matching level), but that if two or more systems have the same functionality, then they will buy the system with the lower 
total cost of ownership. As these are mutually exclusive logic propositions, one alternative of logic must be chosen. 


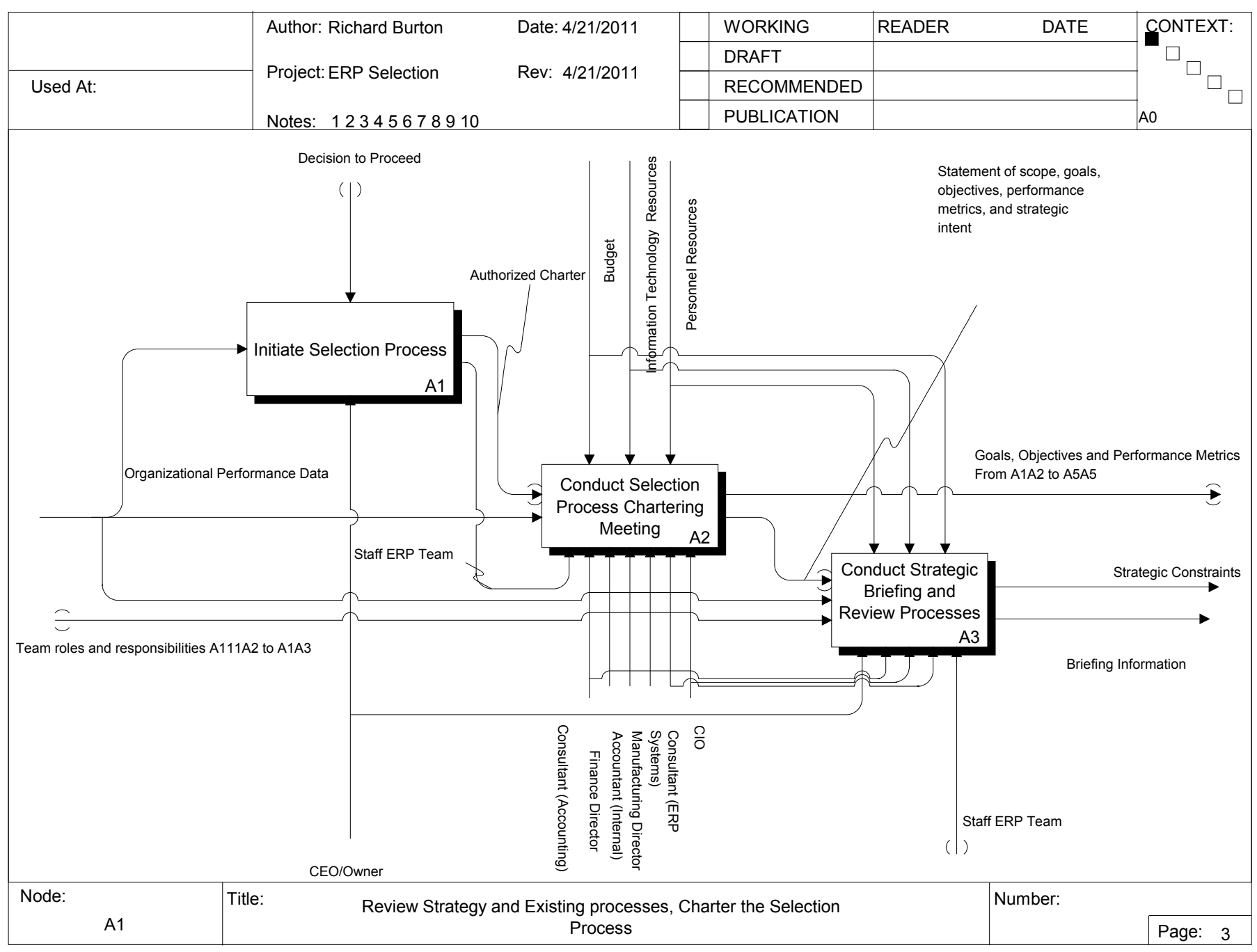

Figure 7 - Diagram A1 Review Strategy/ Charter Selection Process 


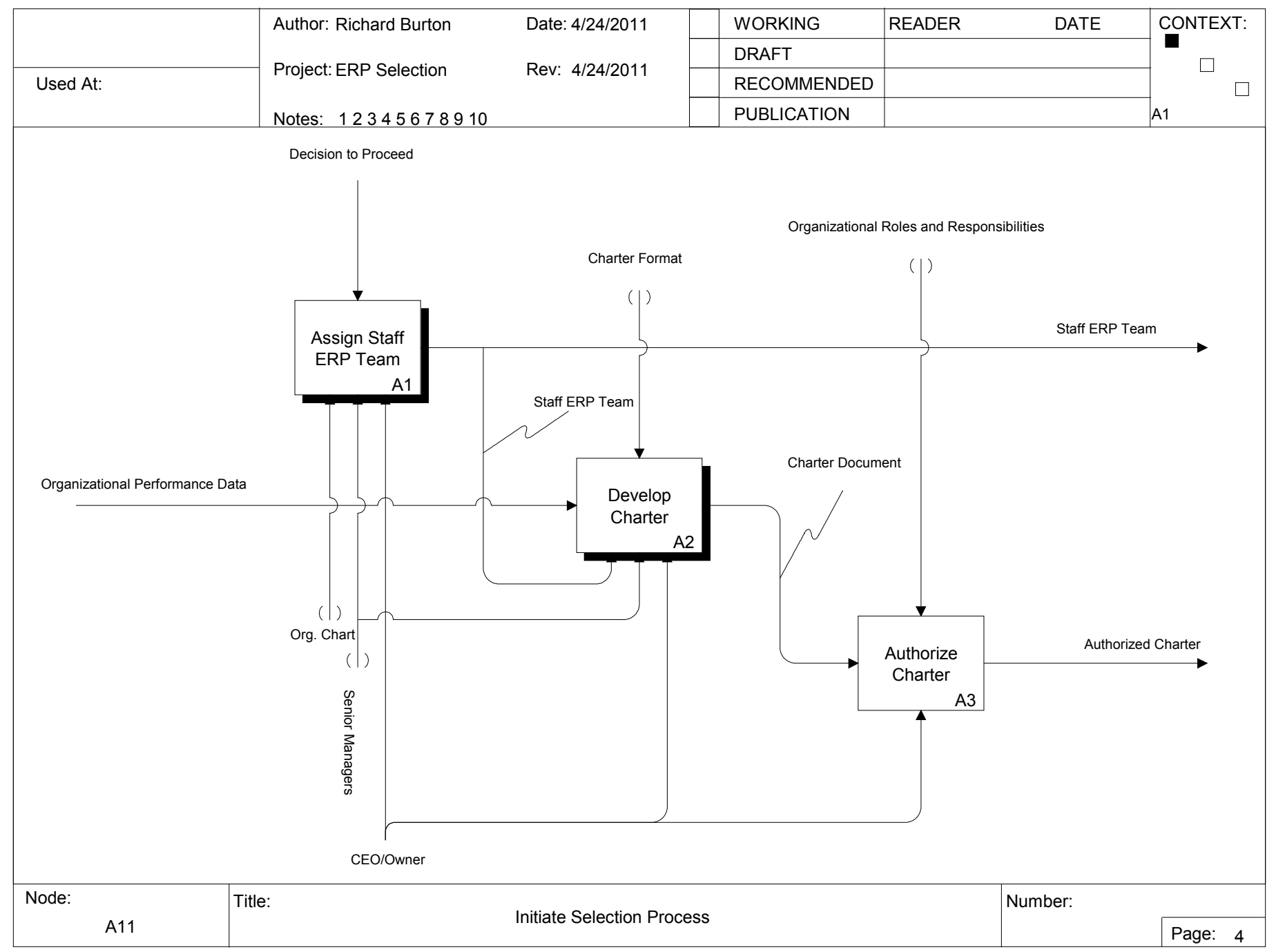

Figure 8 - Diagram A11 Initiate Selection Process 


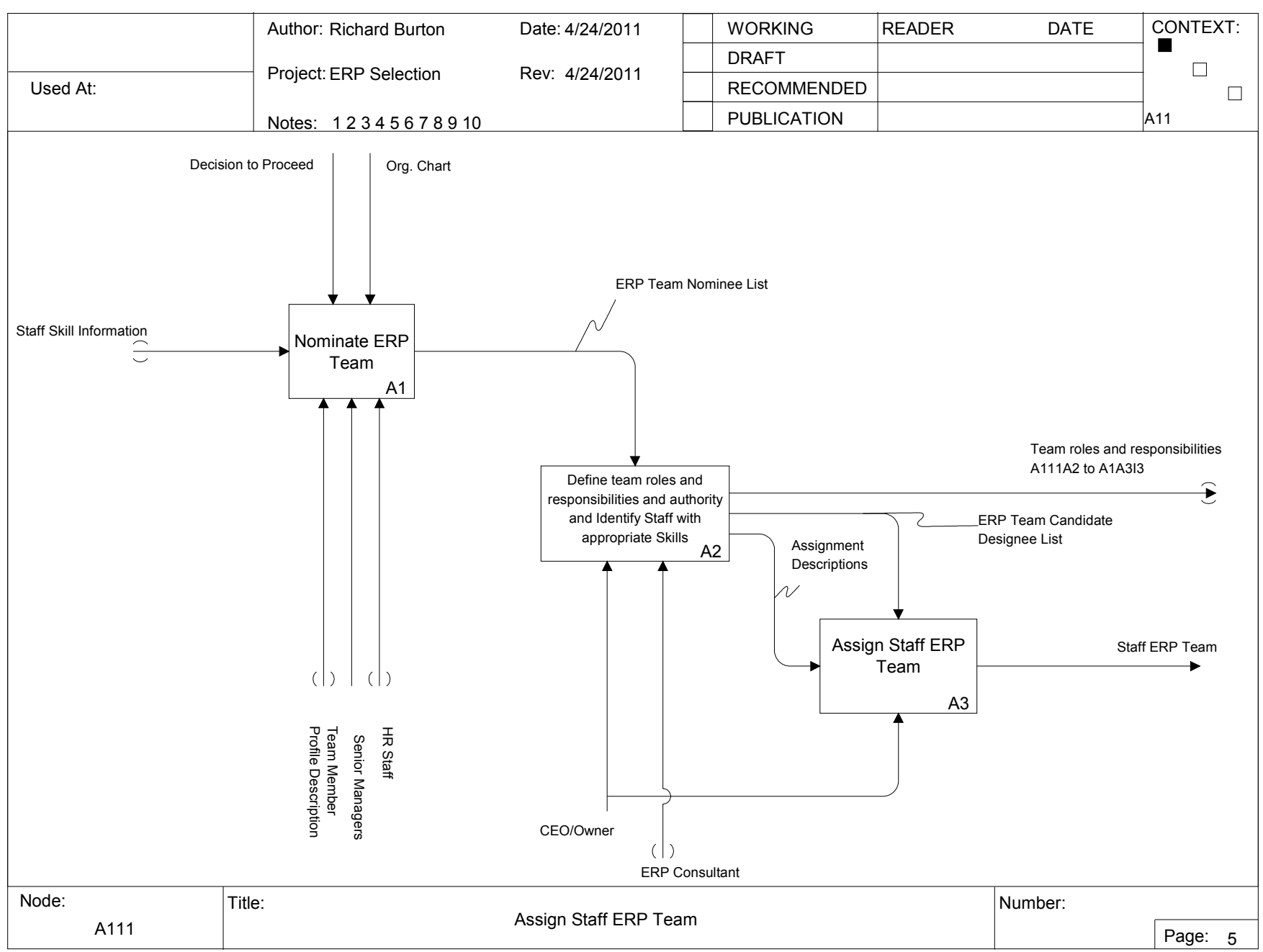

Figure 9 - Diagram A111 Assign Staff ERP Team 


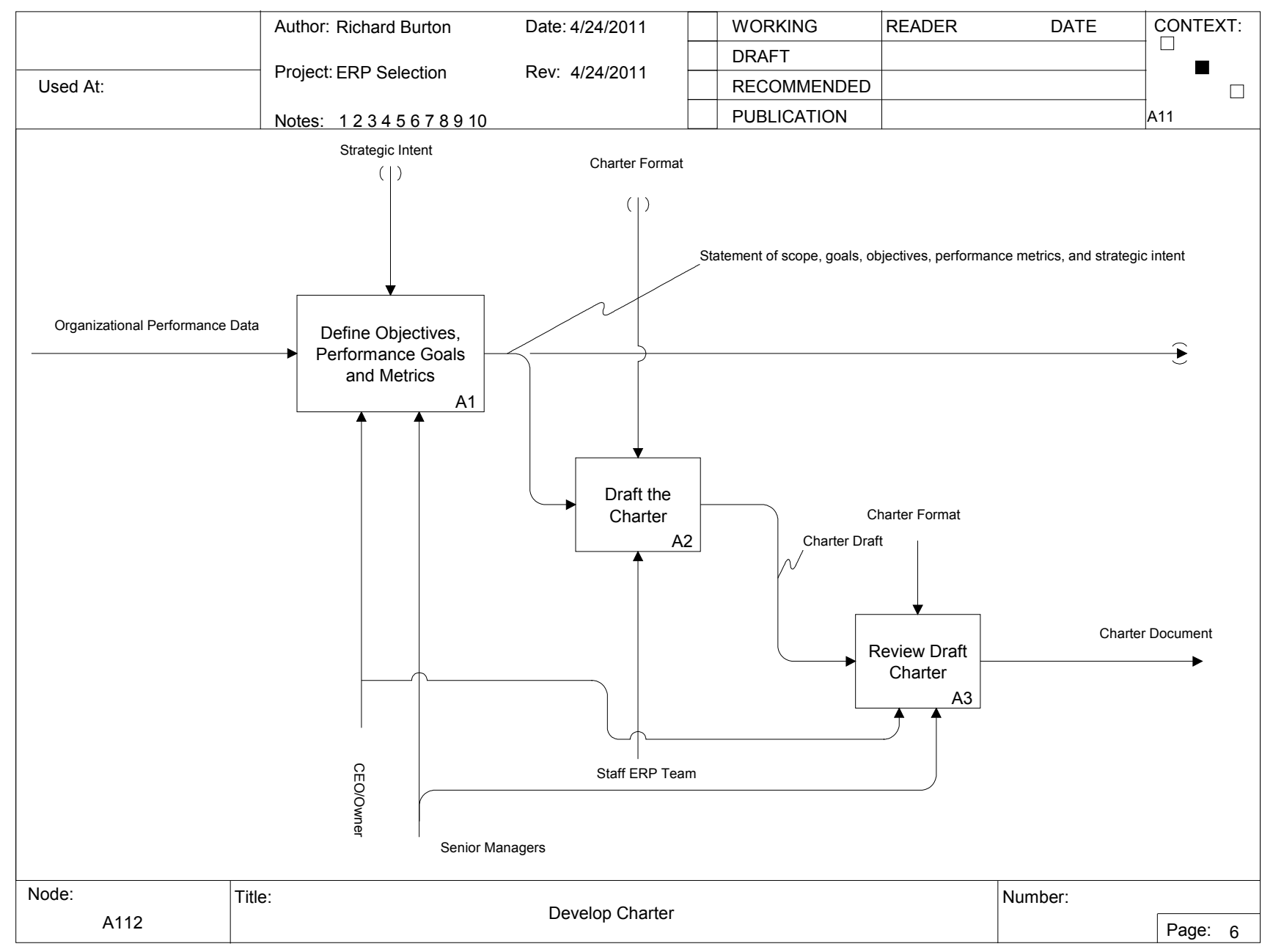

Figure 10 - Diagram A112 Develop Charter 
At the operational level, it is necessary to understand the baseline from which we are starting, and accordingly, we conduct a review of existing company processes and process maps to identify essential features and attributes. For the purposes of our study, we assume that the company is operating at a high maturity level, and that it has captured much of its business process knowledge in the form of process maps, and that any additional processes have been or will be identified in the selection methodology to allow accurate assessment of the working impact, and financial cost of process changes.

We further, determine functional requirements through focused meetings and interviews. The functional requirements for the company are determined on the basis that while the methodology may be applicable to a wide range of business types, each instance of its application will require a specific evaluation tool and that the comparison of requirements to the available ERP system capability is unique to that instance. A process to complete our understanding of the company which captures the strengths, weaknesses, opportunities, and threats is also instructive.

We establish a process to identify any known current business or operational process deficiencies, and to process map them in an acceptable revised form, identifying features and attributes to be added to the future system. Our underlying assumption is that we intend to keep the BPR process to a minimum, but that most instances of ERP system implementation will accommodate changes and improvements that are already recognized within the current operation. In order to amend our baseline, we introduce a 
process element that allows the company to adopt improvements in concert with the ERP introduction.

In parallel, we determine the system selection constraints with reference to both strategic and operational considerations. The initial briefing materials provide the outline of the strategic drivers, and their associated limitations on the selection process; it is necessary therefore to blend them with the operational constraints which must be considered within the selection process.

The objective at this stage is to clearly define the characteristics of the intended business and operational processes that will be in place once the ERP system has been selected and implemented. It allows a review, and definition of all requirements, and allows for a common understanding of the constraints that are in place with respect to the selection and operation of the new ERP system. It has an effective purpose of providing broad participation in the selection process, and underpins the buy-in process (socialization) for the new ERP system among all personnel. 


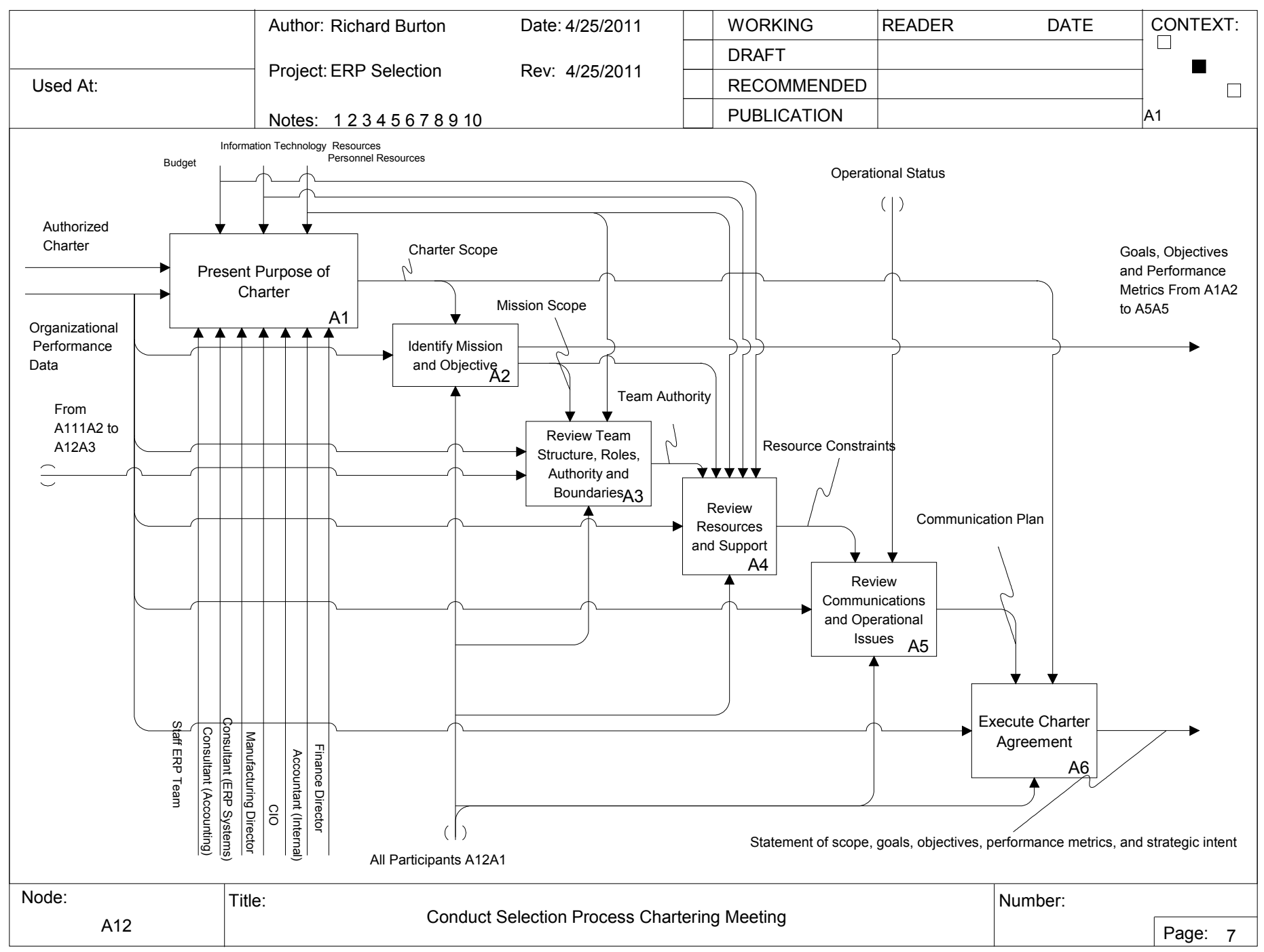

Figure 11 - Diagram A12 Conduct Selection Process Chartering Meeting 
Accordingly, a chartering process is undertaken to provide direction, understanding and to put the team in touch with the strategic objectives, and who at the company is the corporate sponsor.

Chartering is commonly divided into several functional elements, which are incorporated into the process model presented in this research work. The essentials are; understanding the purpose of the work; defining the mission and objective of the team's effort; the structure and roles of the team; the authority of the members; the resources and support infrastructure that is assigned to the effort; how the team and program will operate; and finally, a formal process to negotiate and agree on the specifics of these issues before undertaking the task.

The team should be composed of members with experience and skills appropriate to the tasks at hand, and form a range of different skill areas. It is essential to have a team of the right size, the span of control for a team leader is traditionally determined to be no more than eight individuals, and should involve all functions or departments affected by the new system, and from a range of levels of employee. Where there are apparent gaps in skills or knowledge, consulting resources are clearly advisable additions to the team. The team leader should be responsible for; ensuring the charter is observed, managing day to day team functions, ensuring resources flow to the functions and activities when needed, and communicating the status of the program to managers on a periodic basis. The charter should be a formal living document that is credible, and is developed through a process of agreement and consultation with all parties. All members and stakeholders 
should sign the charter, as a symbolic gesture of commitment and understanding of the mission and its objectives.

It is crucial that the executives, managers, selection team, and future primary users of the new system are fully informed about the objectives, and intent of the selection process. In order to facilitate that process, an extensive level of understanding of the business, its market position, operational constraints, and the return on investment that is expected from the new system are essential. Thomann refers to this process a putting the user in touch with the purpose. 


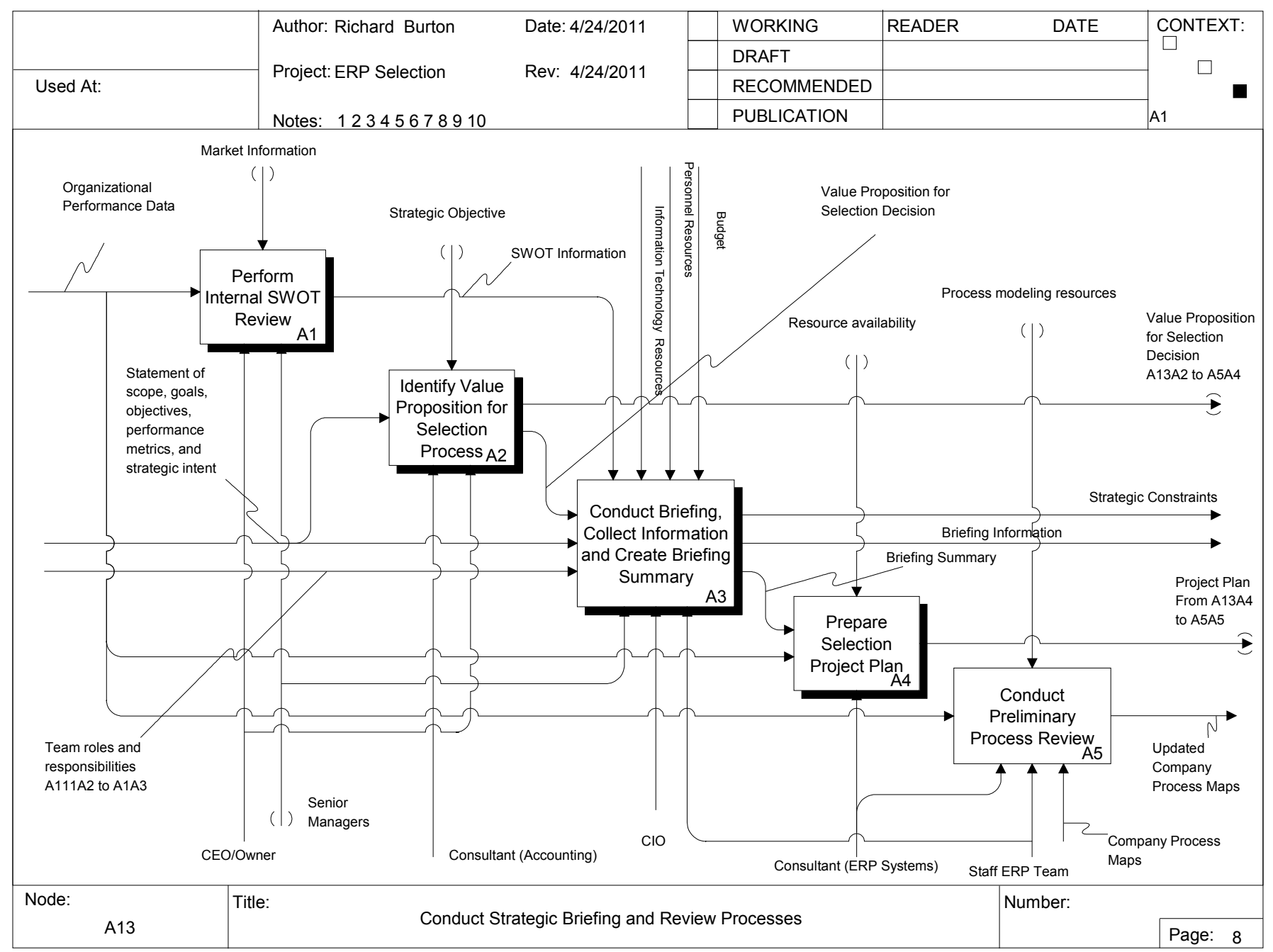

Figure 12 - Diagram A13 Conduct Strategic Briefing and Review Processes 
The intent of the strategic briefing is to condense as much information about the company as possible into a tool for understanding and applying that understanding in the selection process. The information obtained throughout this process, straddles the as-is company configuration, and accommodates a small degree of development in preparation for a transition to a new operating paradigm. Two systems engineering techniques lend themselves to developing this understanding; SWOT analysis, and business process mapping. A third technique, project management, is adopted as a tool for controlling and managing the risks inherent in the undertaking.

The SWOT technique refers to an internal and external analysis of the Strengths, Weaknesses, Opportunities and Threats facing an organization. When employed as a strategic tool, the SWOT analysis can help in developing a global understanding of what needs to be done to maintain and advance the company's business position. Typically, the process is conducted by managers with knowledge of key issues of the company's business posture, and is operationalized by answering key questions from both an internal and external viewpoint.

Key questions to determine strengths include:

- What are the essential distinguishing advantages of the company?

- What are the resource advantages the company benefits from?

- What marketplace issues allow the company to out-compete its competitors?

- How is the company unique?

- What do others see as the company's advantages? 
- How do we succeed when others fail?

From a similar global view of the company, weaknesses are identified by asking:

- What issues have caused us inefficient use of our resources?

- What needs to be improved in our operations, administration or services?

- What do others see as our weaknesses?

- What factors allow others to out-compete the company in the marketplace?

Opportunities are assessed by asking:

- How is our market changing?

- What market opportunities are apparent?

- What technology or service developments are served by our expertise?

- How is the regulatory environment changing?

- What market demand changes are advantageous to our company?

The threat environment is similarly analyzed by answering questions such as:

- How are our competitors changing?

- Why are our competitors out-competing us?

- What financial factors, internal and external are affecting our company?

- Are we industry leaders or do we lag behind our competition?

- Do we need to adopt new technology?

- Do we apply appropriate standards and methods to our work?

These questions are representative of the questions to asked, but the process should be rigorous, and honestly applied, and should be a comprehensive process. 
The objective of the briefing activity is to provide an overview of the program to the team and participants. The content includes the details of the program, an outline of the background, project objectives and the strategic objectives. It also includes information on the timescales for action, the benefits expected and the costs anticipated. In addition, the briefing will propose an outline of how the project will be conducted, what assumptions and constraints are applicable to the project and a discussion of the risk issues for the project. 


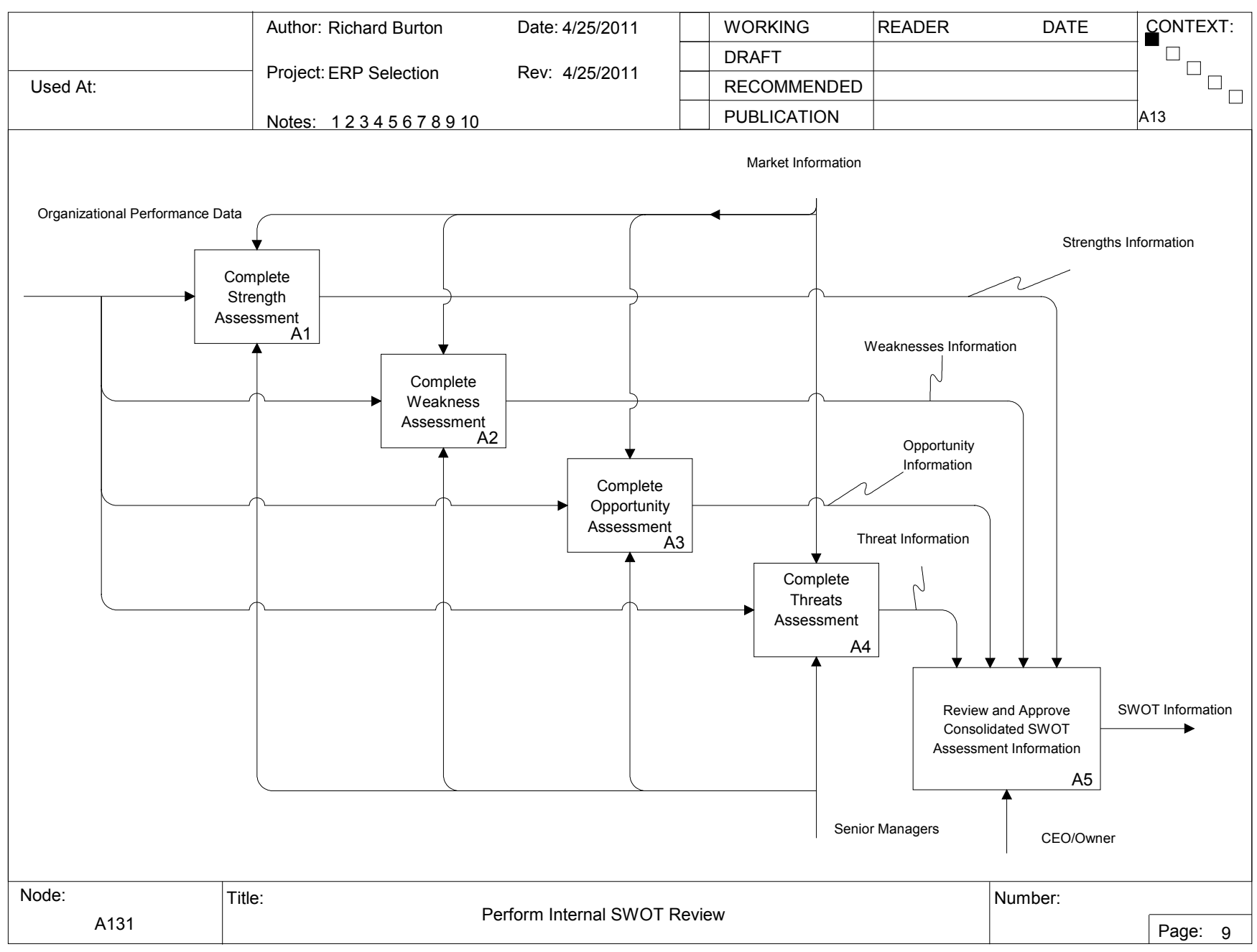

Figure 13 - Diagram A131 Perform Internal SWOT Review 


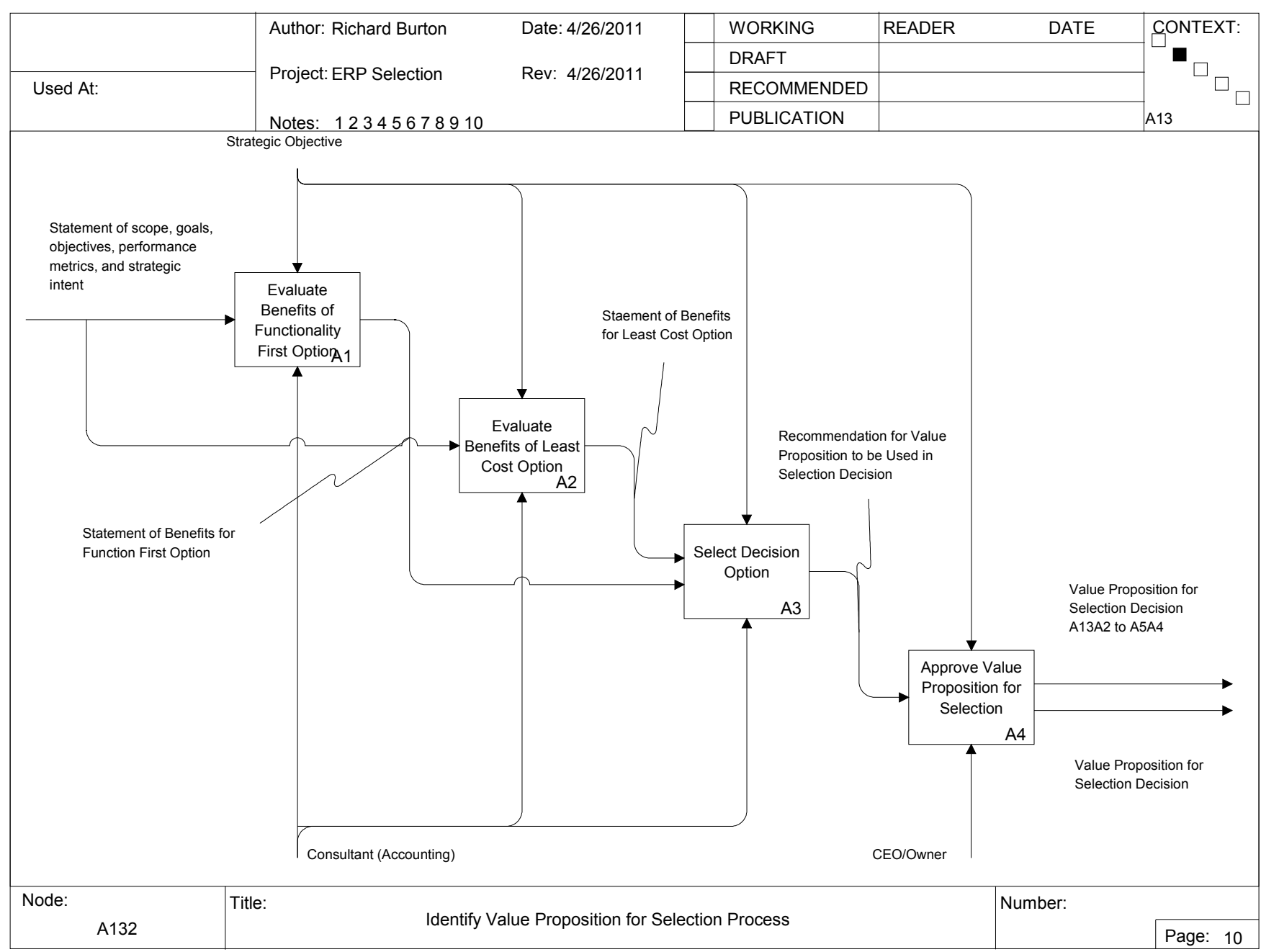

Figure 14 - Diagram A132 Identify Value Proposition for Selection Process 
The briefing consolidates the information required for more formal project planning to occur, and identifies the information which is incorporated into the project plan; tasks, milestones, deliverables. In addition, more detailed estimates are facilitated allowing analysis of task interdependencies, the duration of work elements, critical deadlines, and a preliminary assessment of who should be assigned to which tasks.

Depending on the scale of the effort, project planning tools may be as simple as MS Excel timeline charts, while more extensive projects may require more formal software solutions like MS Project@. The objective of the project plan is to manage risk, control resources, and maintain the timeframe and quality of the decision process. Equally important are the elements of project controls which monitor the cost and track the progress of the overall project and its sub-tasks.

Two project software tools are proposed for inclusion I the methodology, a standard Gantt chart for planning and predictive analysis, and a project dashboard, implemented as a "stoplight" chart in which the real time status of active project tasks are tracked. The dashboard provides an instantaneous assessment of the project's active tasks as well as providing a view of the risk status of any given project element. 


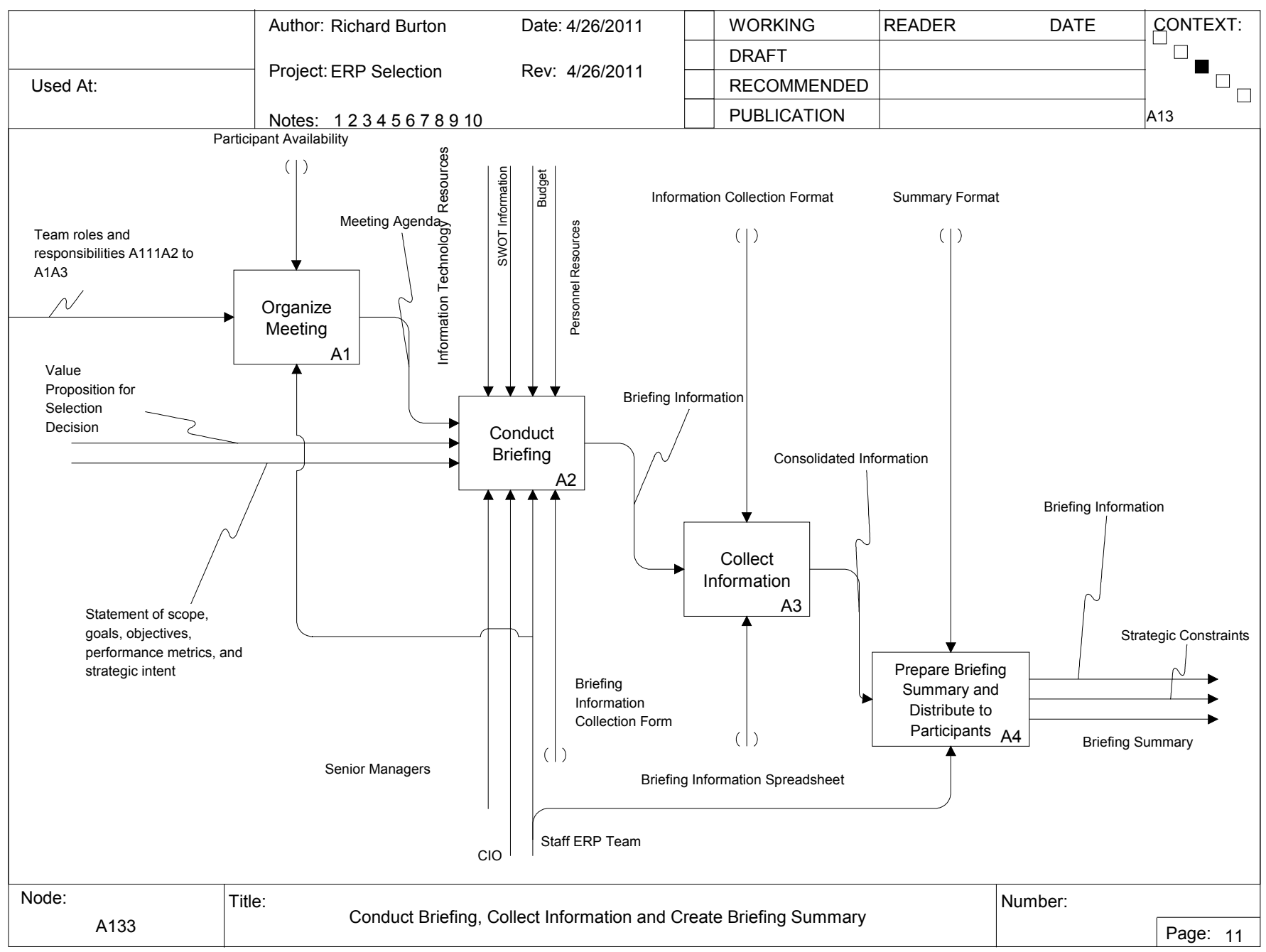

Figure 15 - Diagram A133 Conduct Briefing 


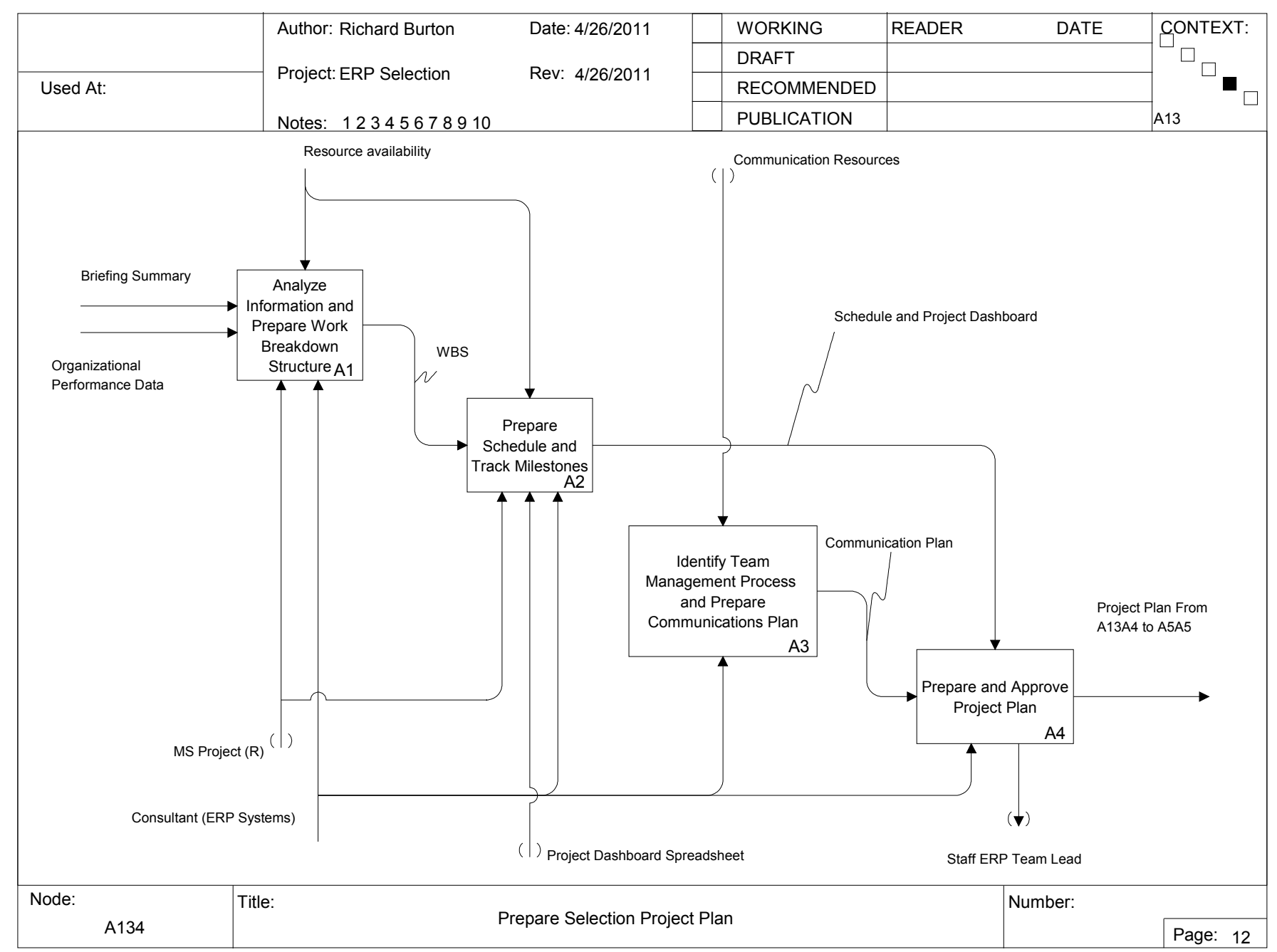

Figure 16 - Diagram A134 Prepare Selection Project Plan 
Understanding strategic requirements is an essential precursor to gathering and understanding the operational requirements. Requirements, in general, are the core elements upon which the degree of fit of a new ERP system will be assessed. They represent the needs which when filled, will improve the company's operations in the ways envisioned in the objective statement.

Requirements are gathered in a variety of ways; through formal brainstorming sessions, by analyzing the company and its existing processes, by applying the information garnered from the SWOT process, and through formal requirements gathering sessions such as Joint Requirements Planning (JRP).

The participants in a JRP session should include; departmental everyday users, occasional users, future users, managers and senior managers. The breadth of participation in this process serves to ensure that the widest set of perspectives is accessed, and that the buyin process of adopting a new system is begun.

The JRP process provides a structured method of collecting this information, it facilitates a wide ranging analysis with the feature that standard and unusual requirements can be proposed; and it provides an environment in which all issues and concerns that are related to the requirements specification process may be addressed.

The process allows the breadth of analysis that is necessary for a comprehensive analysis, and allows iteration and reconsideration of requirements as they are built. This approach 
allows the requirements to be clarified at the high level that is needed to allow them to serve as the evaluation basis for the new ERP system.

As the iterative process proceeds, the requirements will resolve and their relative priorities and importance will become apparent. Another advantage of the JRP technique is that it quickly identifies problem areas for the selection process; any lack of support from users or sponsors can be identified, the efficacy of the management plan for the selection process will become apparent, and any lack of clarity in objectives will be demonstrated. If these issues do occur, mitigation or correction plans can be made to address the problems early.

A full understanding of the technical, information technology environment should be captured through a selective interview process with management and technical staff. This element is essential to developing the overall request for information and acts as a guidance tool for ensuring that the introduction of the ERP system does not engender unnecessary hardware upgrade costs. 


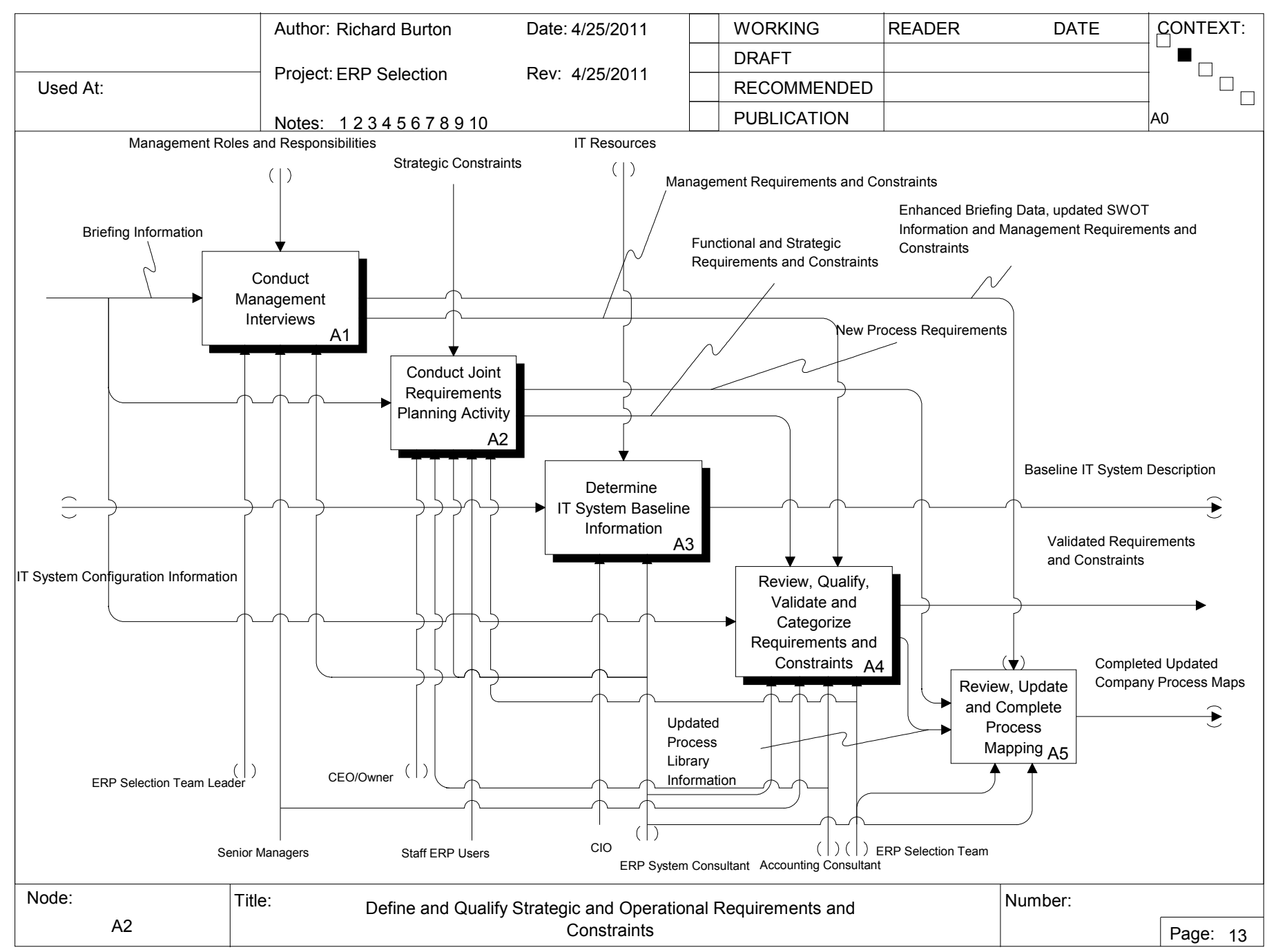

Figure 17 - Diagram A2 Define and Qualify Strategic and Operational Requirements and Constraints 


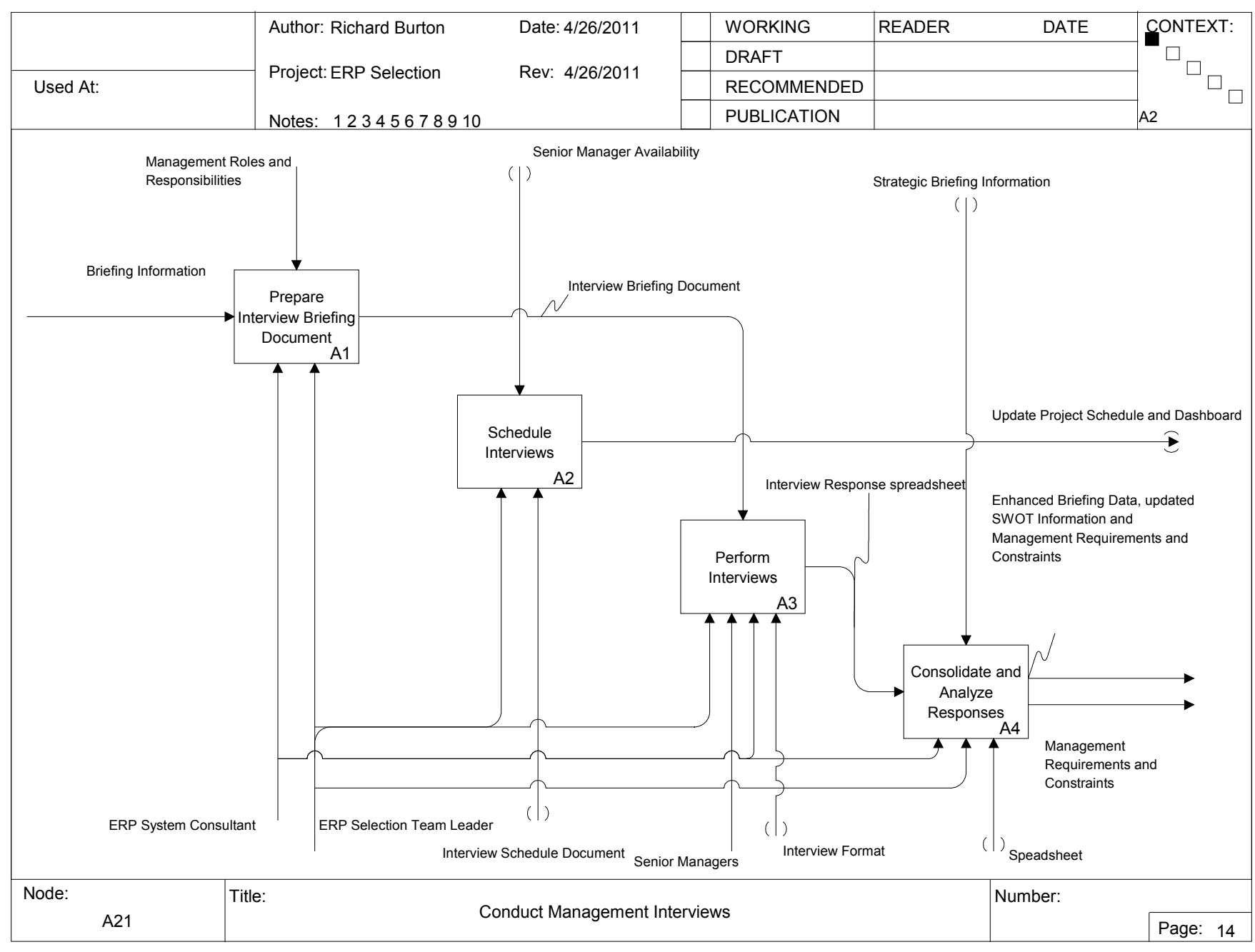

Figure 18 - Diagram A21 Conduct Management Interviews 
In order to match the company requirements to the ERP system capabilities, with a view to selecting a best value answer, we begin with the identification of all the ERP systems that are available. The outcome of this process is to create an initial long-list of candidate systems. At this stage an RFI or similar instrument is issued to obtain preliminary performance, structure and cost information about the available ERP systems.

Procedurally, we pre-sort the RFI respondents, to identify qualified candidate software systems meeting the primary constraints and we create a short-list of candidate systems. This sorting process is informed from various sources including the RFI responses consultant input, and by researching any public domain information that is deemed reliable. Importantly at this stage, we review both the RFI responses, and perform due diligence with respect to the responding vendors, to determine whether there are any business related issues that might otherwise disqualify the vendor i.e. known deficiencies in the product, lack of support services, poor client reputation, etc.. We must also characterize, classify and analyze the requirements so that they can be matched to the capabilities of the short-list candidate group, which will be used to identify the best matches with respect to functional utility factors, and create a finalist list. 


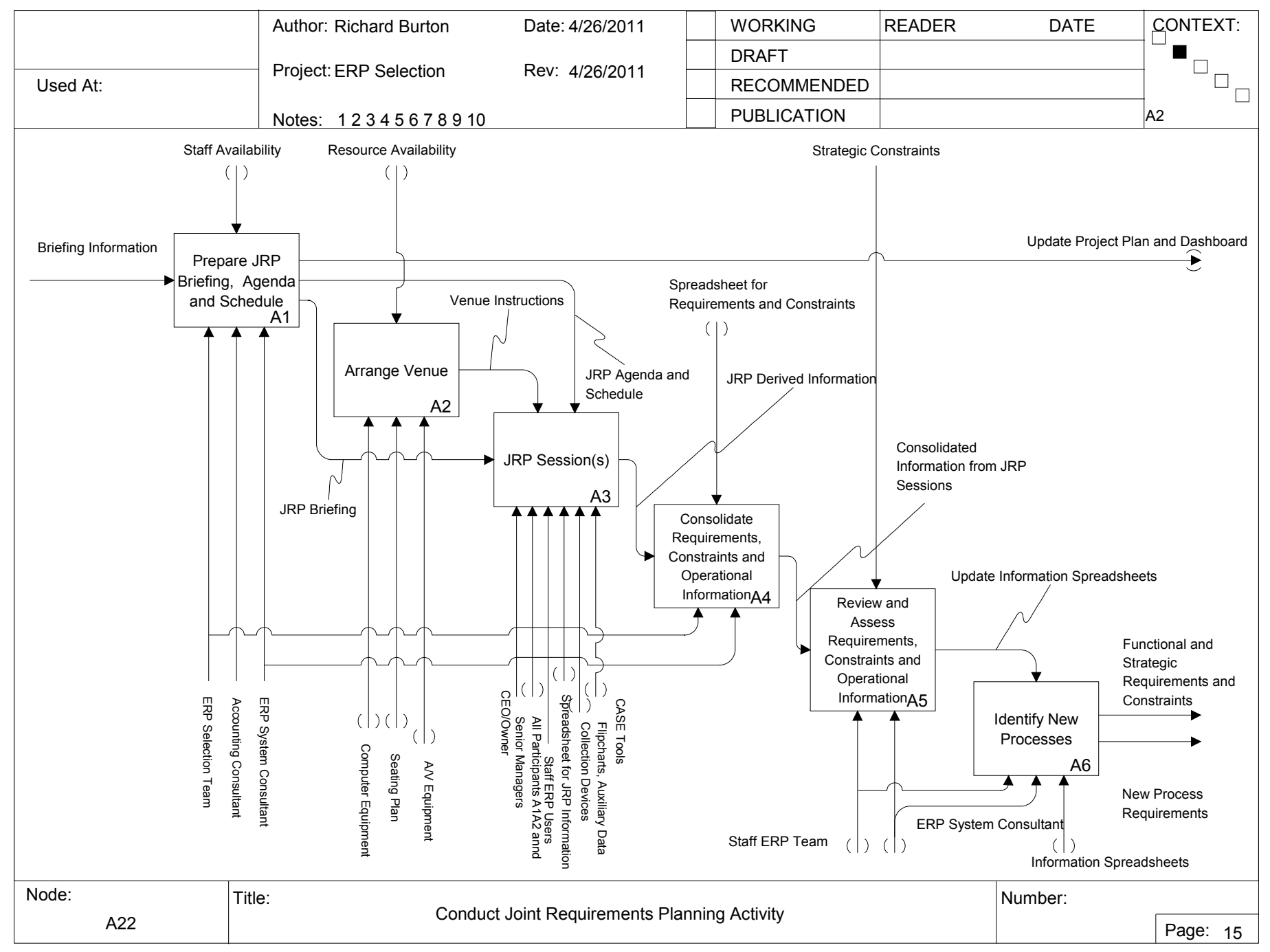

Figure 19 - Diagram A22 Conduct JRP Planning Activity 


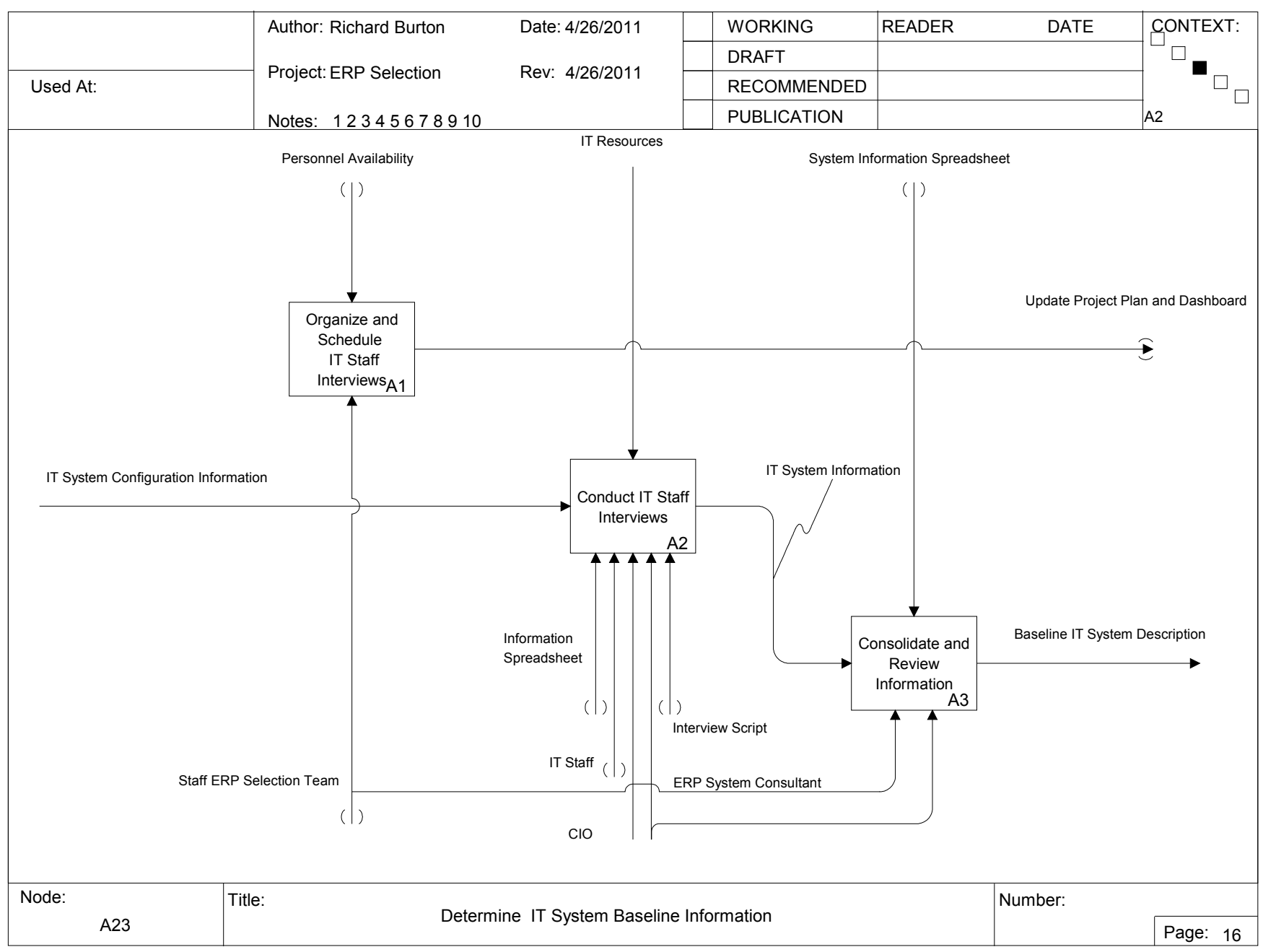

Figure 20 - Diagram A23 Determine IT System Baseline Information 


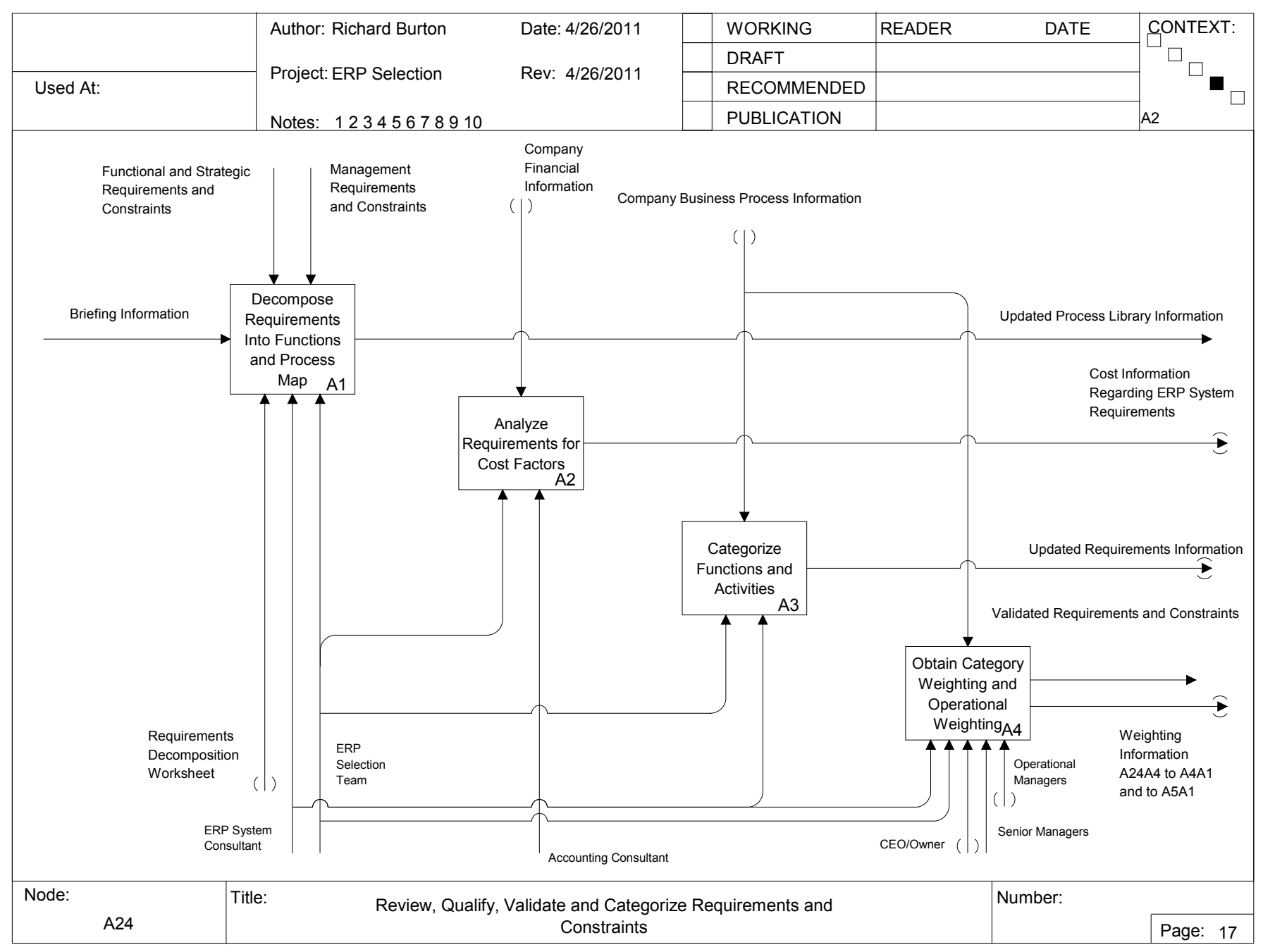

Figure 21 - Diagram A24 Review, Qualify, Validate and Categorize Requirements and Constraints 
The identification of available systems is a critical component of a truly objective process, so a good deal of attention should be applied to this process. The search should address as many sources as possible:

- Published materials

- Trade journals and professional articles

- Internet based searches

- By reference to other organizations who may have undertaken the process

- Consultants

- Etc

The process is likely to involve large amounts of data, so care is taken to design data capture tools, spreadsheets etc, which allow all information to accessed and sorted in a variety of ways, to aid in the later analysis and in the filtering that is to take place. Contrarily, once all the data has been assembled the next step is to abbreviate the candidate list by rejecting the obviously inappropriate vendors and systems, while retaining the systems which either marginally or fully appear to meet the needs. Documentation should be maintained to ensure that the reasoning for accepting or rejecting a system is retained as project knowledge, and to ensure that all findings and conclusions are justified and reasonable. These processes lead to a discriminated list of highly likely vendors and systems for the requirements we identified earlier. 


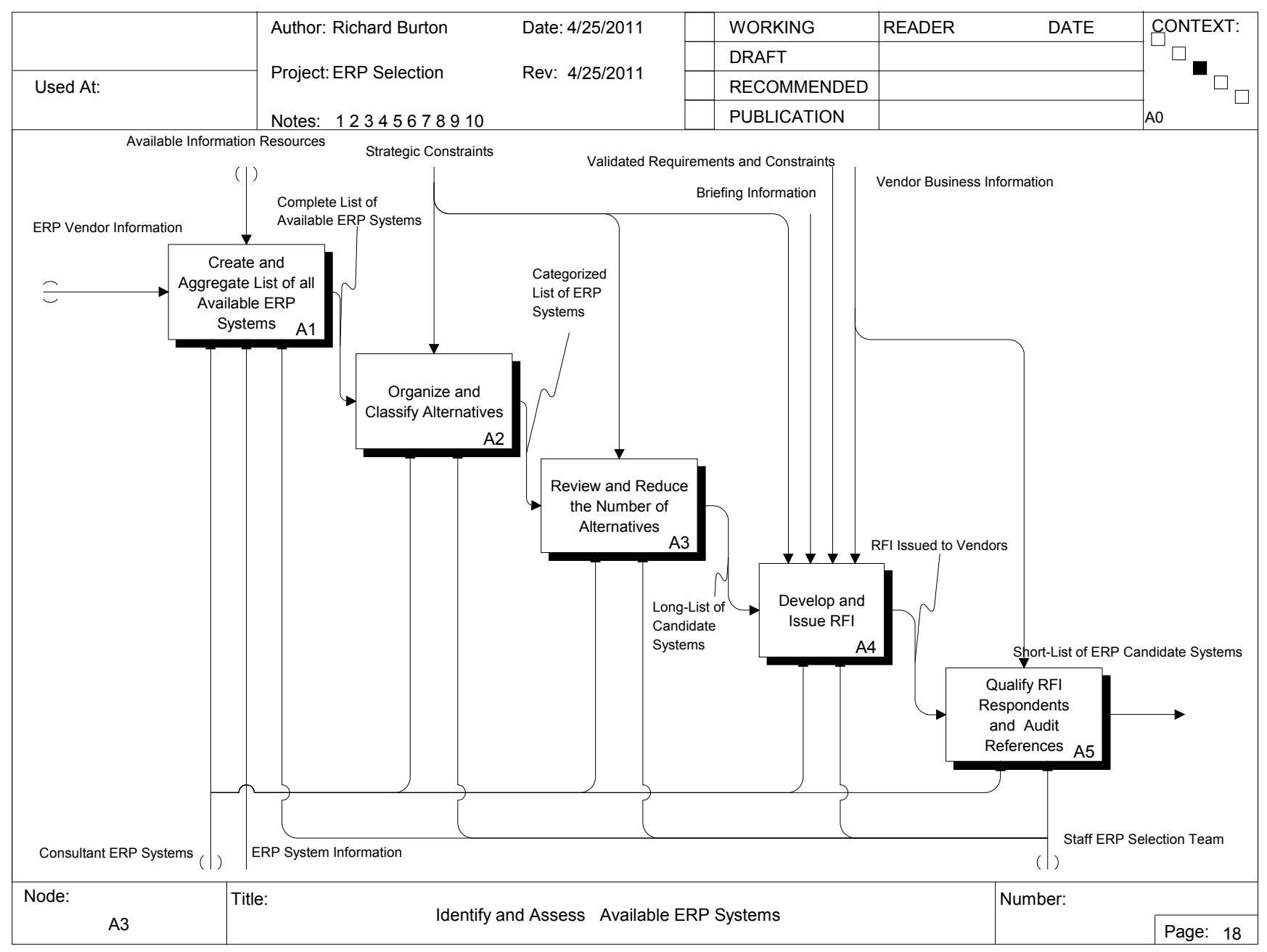

Figure 22 - Diagram A3 Identify and Assess Available ERP Systems 
It is important to identify whether requirements are current functionalities or new functions, principally to ensure that the baseline operating capability is retained before system enhancements are considered. In order to achieve this, the requirements must be decomposed into component activities, by process mapping or other techniques. This leads to the analysis of the requirements, which is conducted in order to; determine who performs or should perform each activity associated with the process, identify workload changes, assess training requirements, review and identify any new staffing requirements. The analysis also allows a categorization of the activities into logical groupings reflecting the business model employed by the company.

As we proceed, it is necessary to obtain an assessment of the relative importance of each category of requirements in a company specific strategic level category weighting assessment, which is a primary input to the evaluation process. In addition we now obtain an assessment of the absolute importance of each activity within each requirement category, an operational activity weighting assessment. The operational weighting is also informed by performing a benchmarking survey to reflect industry experience, and ensure that best practices are identified and incorporated into the valuation scheme. The evaluation process that is adopted must determine whether each activity within the requirements is addressed by a corresponding capability in the ERP system being considered. Our objective is that for each ERP system that is short-listed we accumulate an evaluation of the total functional capacity with respect to the categorized activities, and that we identify any unaddressed needs in each software system. This process will allow us to rank the ERP systems under consideration by their total functional capacity 
score with respect to the functional needs described by the requirements, and to complete the evaluation process with a rigorous cost evaluation. Our evaluation protocol is one which reviews functional fit first, followed by the application of the costs of ownership to determine the best value ranking.

Obtaining pricing information from vendor companies who are engaged in competition is always a challenging proposition. Most companies will provide some rough order of magnitude pricing at the RFI stage, but the literature suggests a great deal of caution should be exercised with regard to preliminary price quotes (Bernroider, 2001). Consequently, for the short listed ERP vendor candidates, a specific pricing outline should be required to allow true comparison matching when determining the total cost of ownership over a given comparison timeframe.

Based on industry benchmarks and the survey results, the evaluation scheme identifies a five year span as an instructive and reasonable duration in order to understand the total cost of ownership of an ERP system. We issue an RFP to determine the costs of each of the finalist systems over the evaluation base period in order to identify best pricing available on a competitive basis, for a five year cost horizon. The fundamental pricing considerations, which inform the development of the RFP are; the cost of acquisition, the cost of maintenance per year for five years, the cost of implementation process, the cost of adding capability to the ERP system for unaddressed functional needs (customization etc.). 
Other costs, may or may not be costs attributable to the vendor, or may be internal to the company; such as, the costs of training (initial and periodic refreshers and/or new staff training), the costs of consulting support, the cost of IT system upgrades and the costs associated with increased workloads during the implementation and during future operations. 


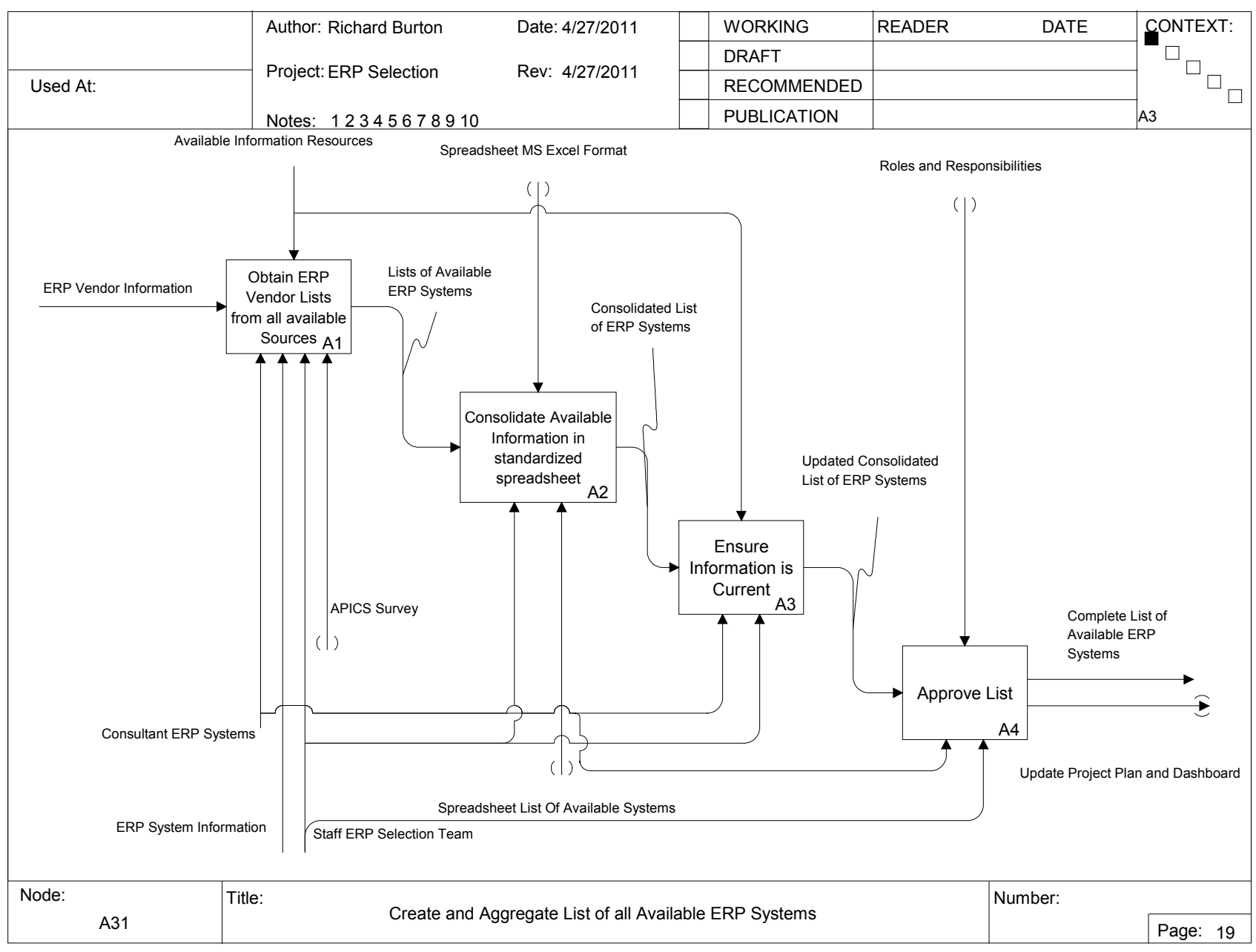

Figure 23 - Diagram A31 Create and Aggregate List of all Available ERP Systems 


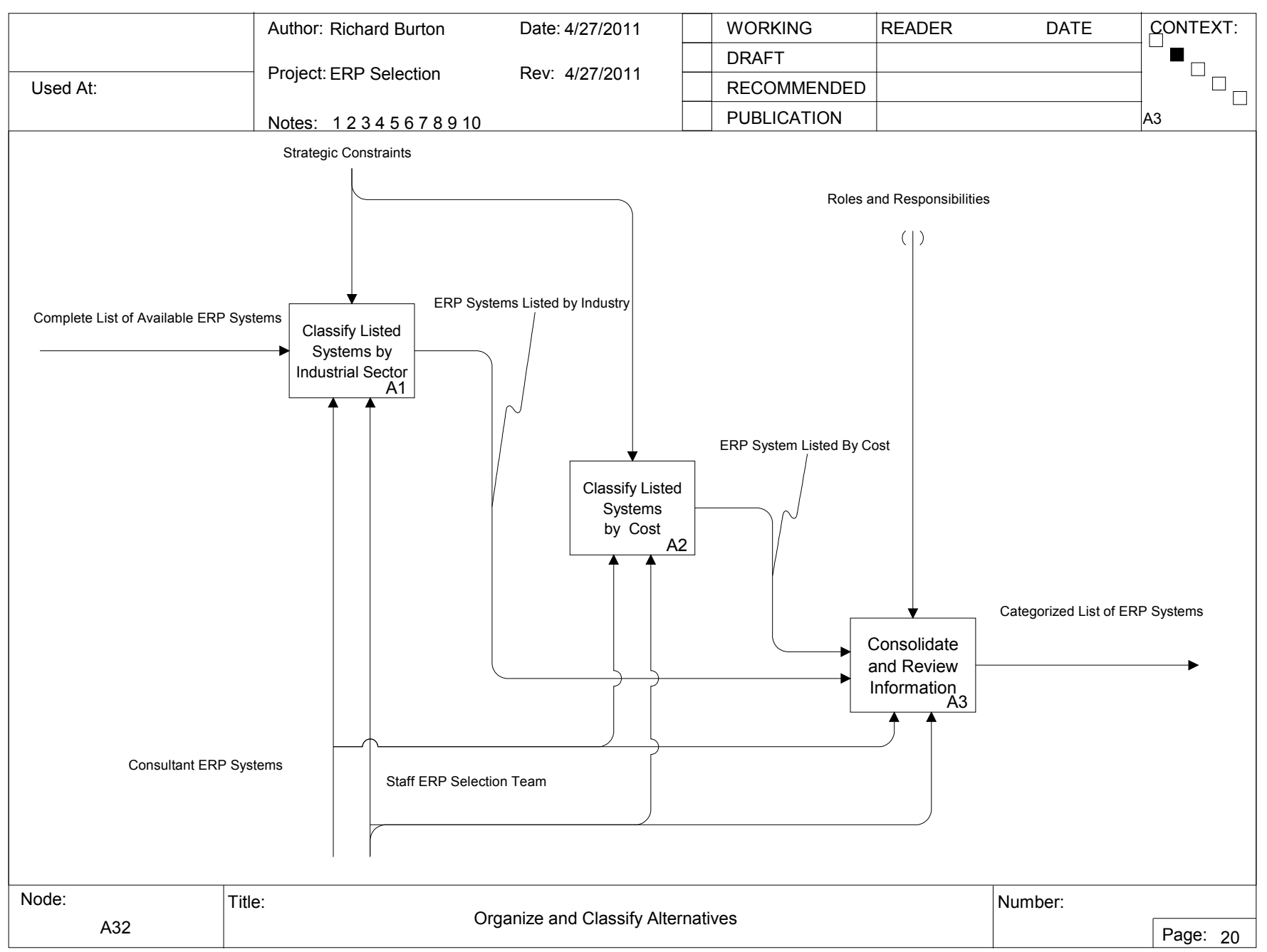

Figure 24 - Diagram A32 Organize and Classify Alternatives 


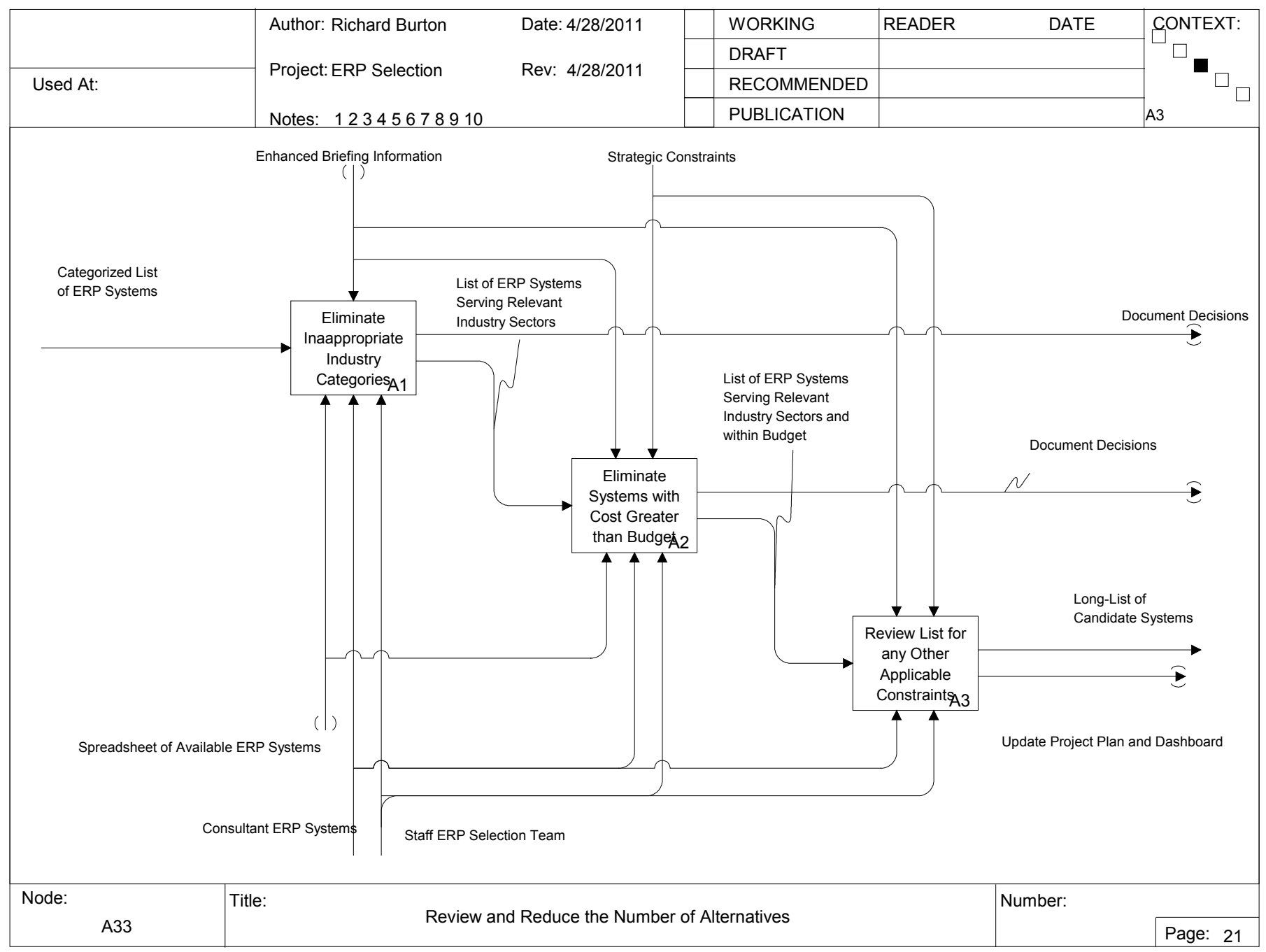

Figure 25 - Diagram A33 Review and Reduce the Number of Alternatives 
The next step in the process requires an initial level of contact with the likely vendors. This may be achieved through a Request for Information (RFI) process. The typical RFI will consist of an overview in which the RFI requests are summarized, and the objectives and benefits being sought are identified. The RFI shares with the vendor the expectations for the duration and path of the selection process, how the selection will occur and the basis for evaluation of the systems. It also shares the vision and expected timeline for the implementation process. We include a confidentiality requirement, as the succeeding process will share business critical information with the vendor.

Contextual information about the company should be provided, including:

- The market sector served by the business,

- Revenue and growth expectations, size and market reach

- An outline of the IT environment

- System users - current and growth target

- Selection team contacts and outline for communication protocols.

The requirement description should contain:

- Assumptions and constraints that are relevant to the vendors understanding of the project

- An outline of the requirements of the company

- Any non-standard requirements or expectations

The vendor should be asked to respond by a specific date and time, with a written response. The response should include a statement of interest from the vendor, 
confirming or declining participation in the selection process. It should also contain an outline of the proposed system and as much detail as possible on how they intend to respond to the technical requirements. In addition, the RFI should seek to identify how many clients the vendor has serviced, what the size of their company is, how mature the solution is, how long the implementation may be expected to last, and their experience in the marketplace with similar clients. The RFI response should explicitly identify references and initial cost information for the system.

When the responses are received, they should be recorded and the receipt should be acknowledged, the selection team should review the response for completeness. Once all RFI responses are received, the team should analyze them using a scoring method, the review should look for and evaluate the level to which requirements are specifically addressed by each response, and a scorecard should be maintained for each system which has been proposed.

Some of the less tangible facets of business reputation are critical to qualifying vendors; it should be determined how strong the vendor is financially, what staff levels are maintained locally? How well are the vendors staff qualified? Is the vendor is an original system provider or a reseller? Is the vendor is an agent the team should ensure that the vendor is currently licensed, who is responsible for warranties? Whether the service staff is local or from a remote location? Whether the vendor can address modification or customization in its own right or through the primary developer? 


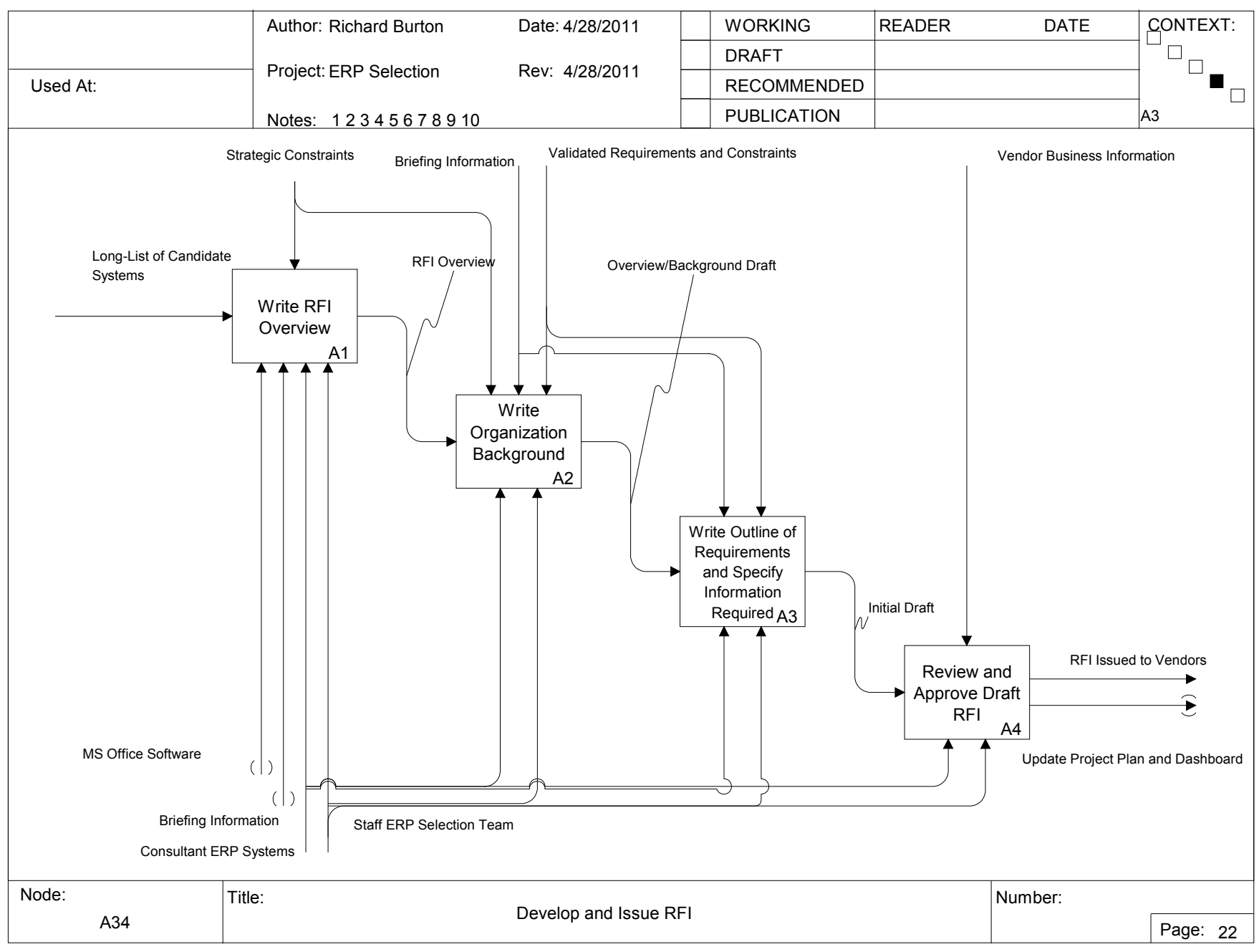

Figure 26 - Diagram A34 Develop and Issue RFI 


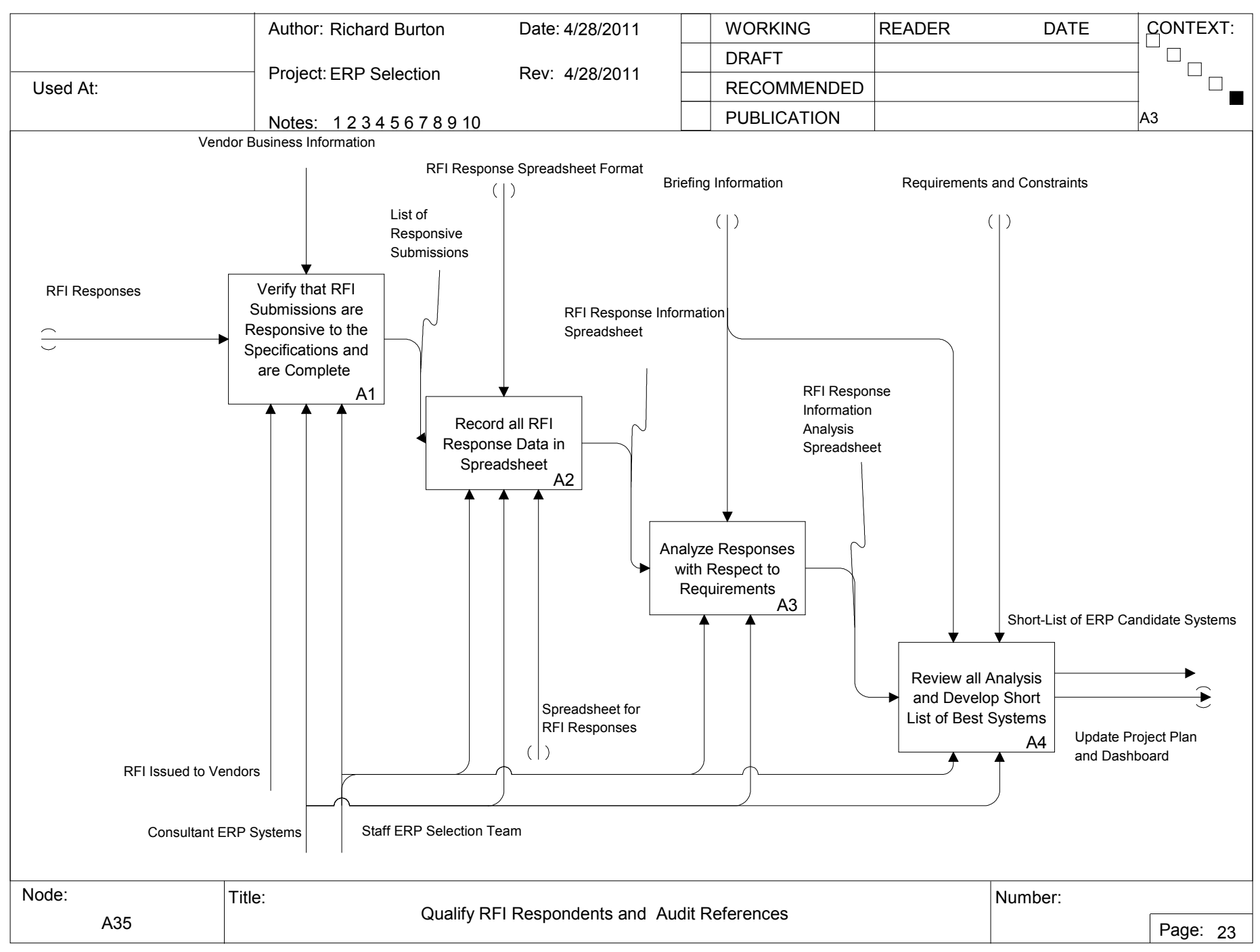

Figure 27 - Diagram A35 Qualify RFI Respondents and Audit References 
In order to evaluate which ERP system presents the best value to the SME, we arrange to conduct a vendor evaluation process for finalist list of ERP systems solutions identified. The product performance tests are developed in order to identify the relative merits of each system and its vendor's capability, and to complete a performance scorecard for each of the finalists. This process allows a standardized performance test to be developed and employed, thereby helping to ensure as objective a process as possible. 


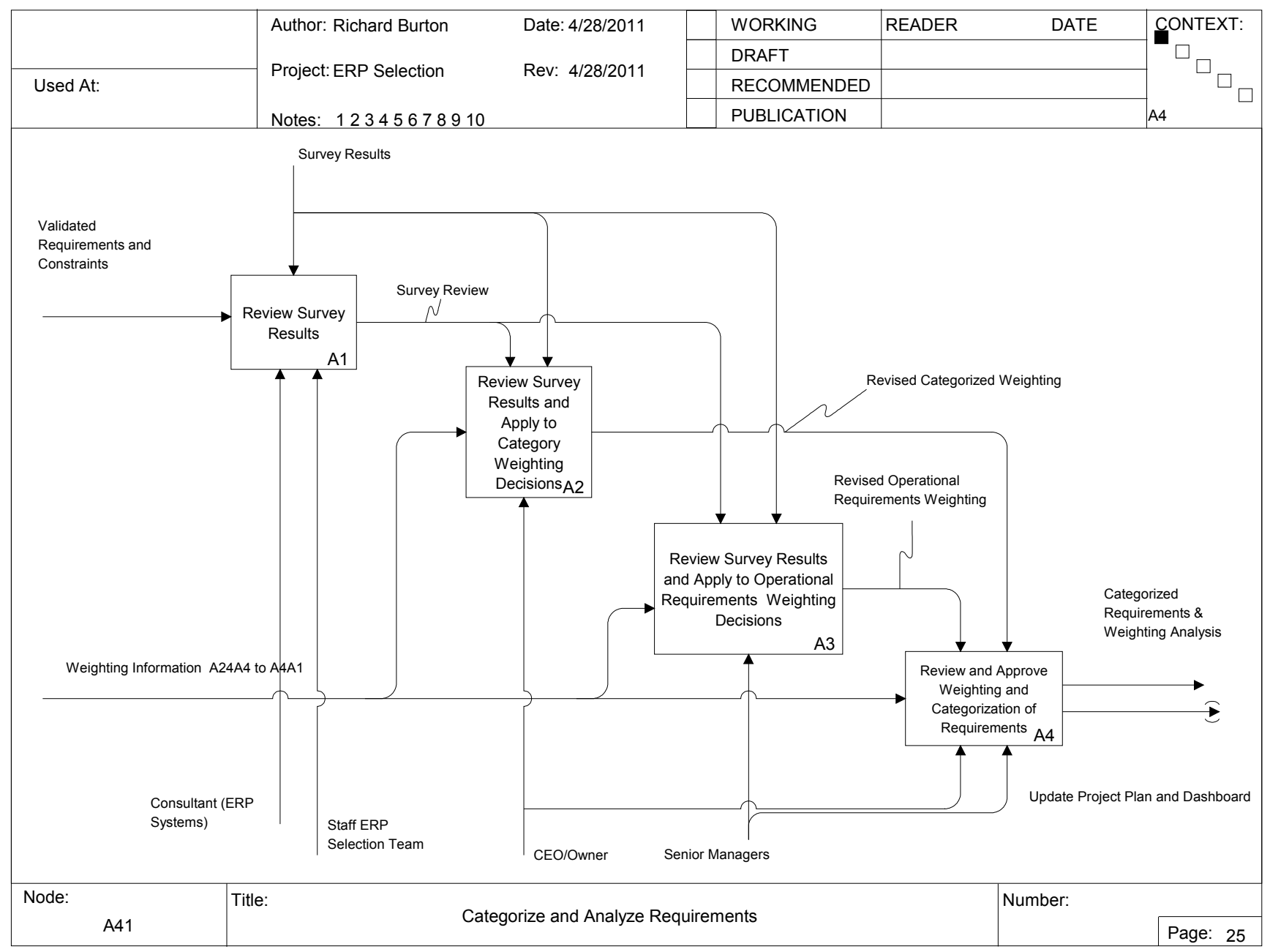

Figure 28 - Diagram A41 Categorize and Analyze Requirements 


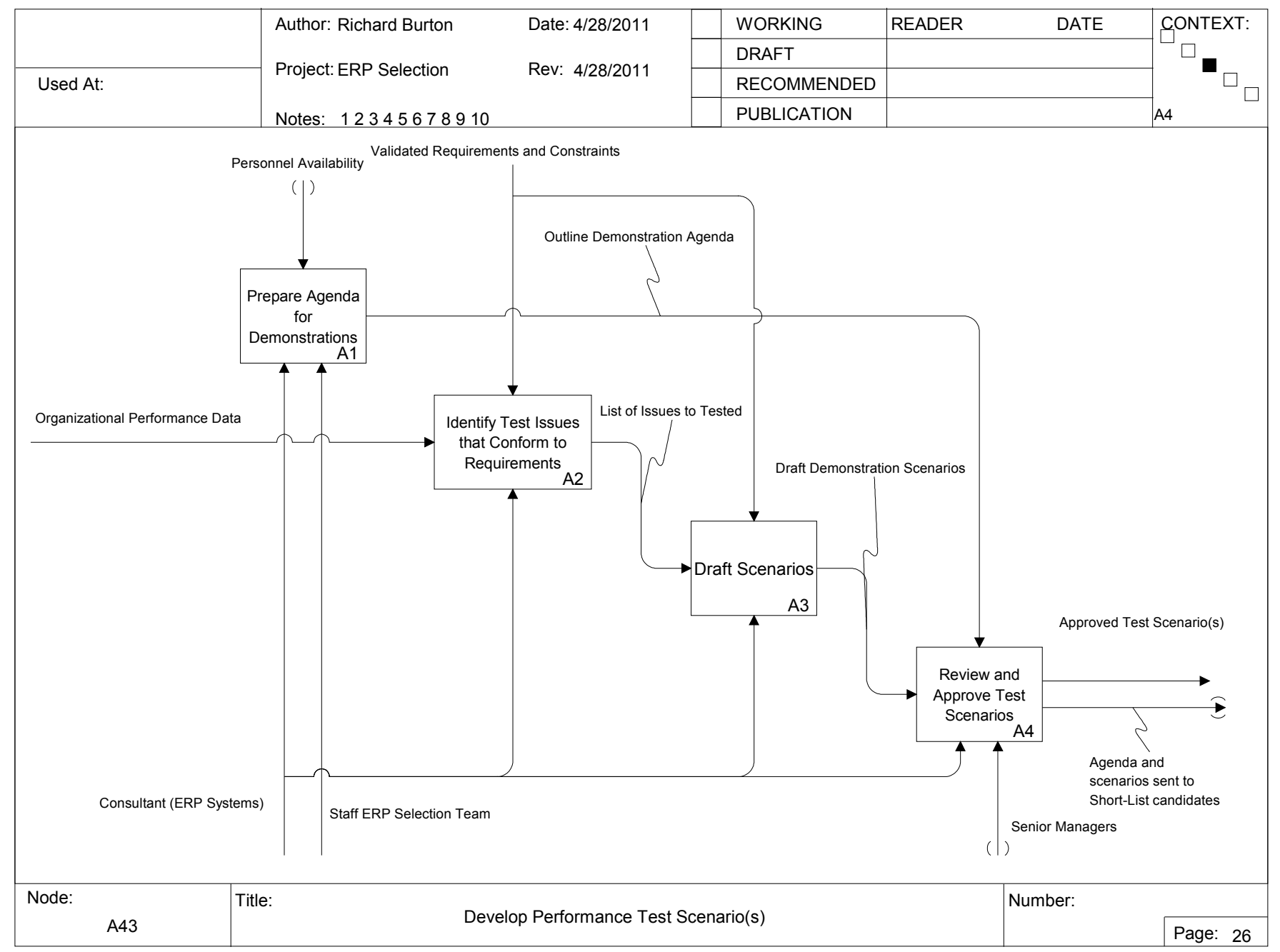

Figure 29 - Diagram A43 Develop Performance Test Scenarios 


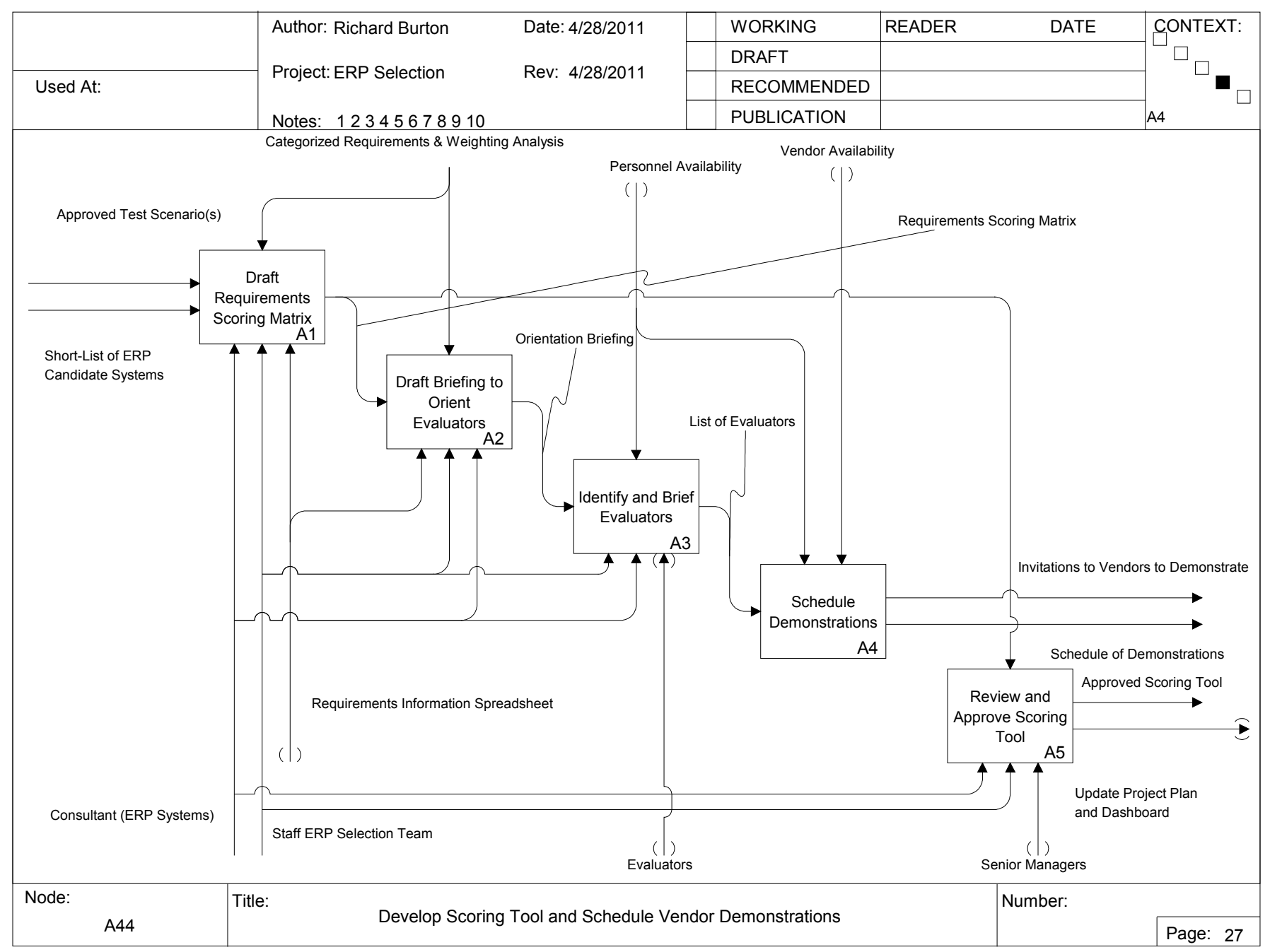

Figure 30 - Diagram A44 Develop Scoring Tool and Schedule Vendor Demonstrations 


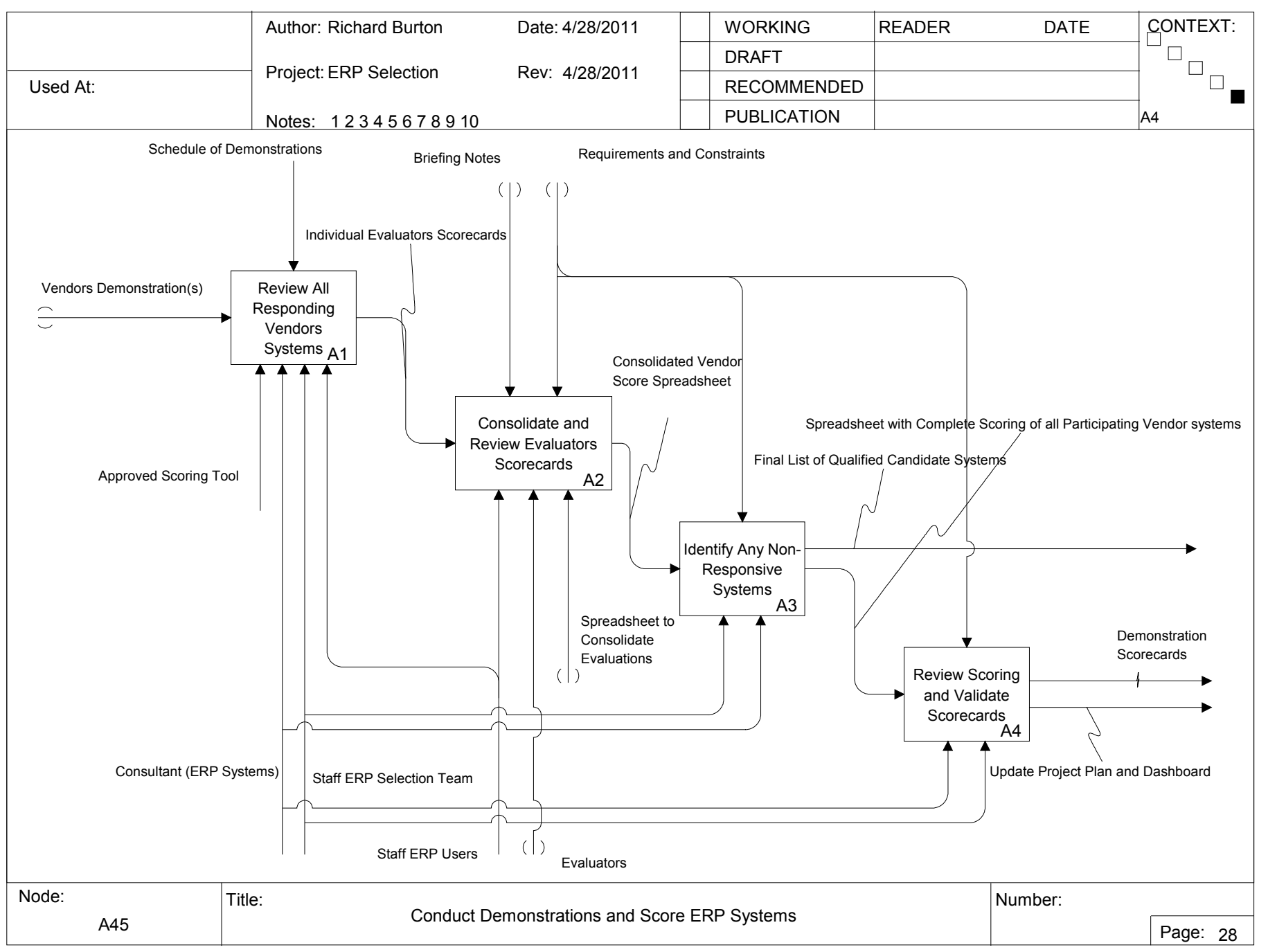

Figure 31 - Diagram A45 Conduct Demonstrations and Score ERP Systems 
At this stage we identify what level of functional capability is minimally acceptable to the specific SME. We apply the best value assessment logic to each of the finalist ERP system scorecards in order to determine; which systems provide at least the minimum acceptable degree of matching of functional capability.

We now apply the logic for selection chosen in the initial briefing. If we desire the lowest cost alternative, with the best functionality, we rank the systems from lowest cost to highest, and pick the least cost system. If the total cost of ownership for the lowest cost of ownership alternative(s) is a tie between two or more systems, the system with the highest functional score is chosen. If there is still a tie we review which system has the best score in the highest rated category of requirements, and pick that system. If there is still a tie we apply the choice at the next category in sequence until the tie is broken.

If we desire the alternative with the best functionality, we rank the systems from highest weighted functional score to the lowest, and pick the highest functional ranked system. If the functional score is a tie between two or more systems, the system with the lowest total cost of ownership is chosen. If there is still a tie we review which system has the best score in the highest rated category of requirements, and pick that system. If there is still a tie we apply the choice at the next category in sequence until the tie is broken. 


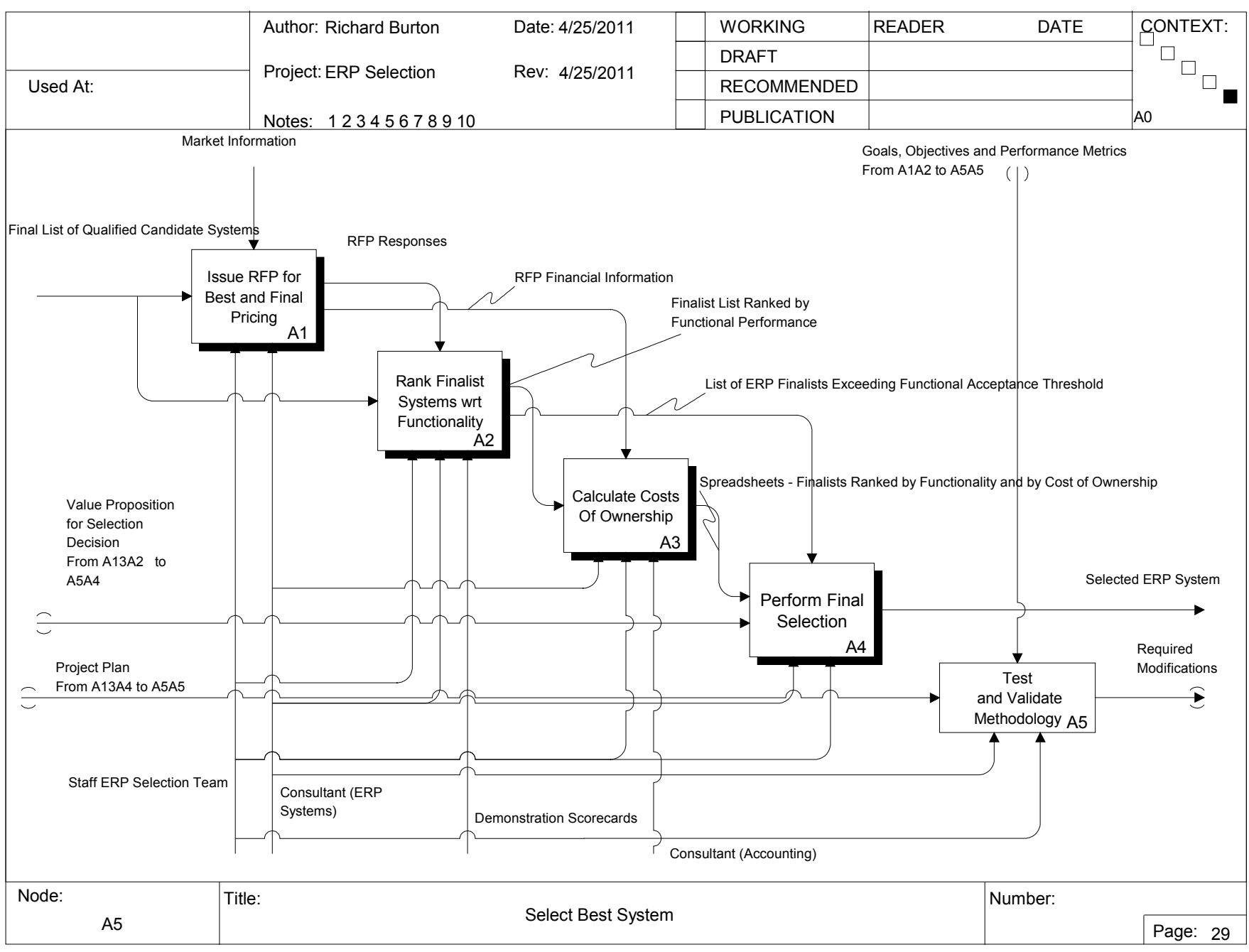

Figure 32 - Diagram A5 Select Best System 


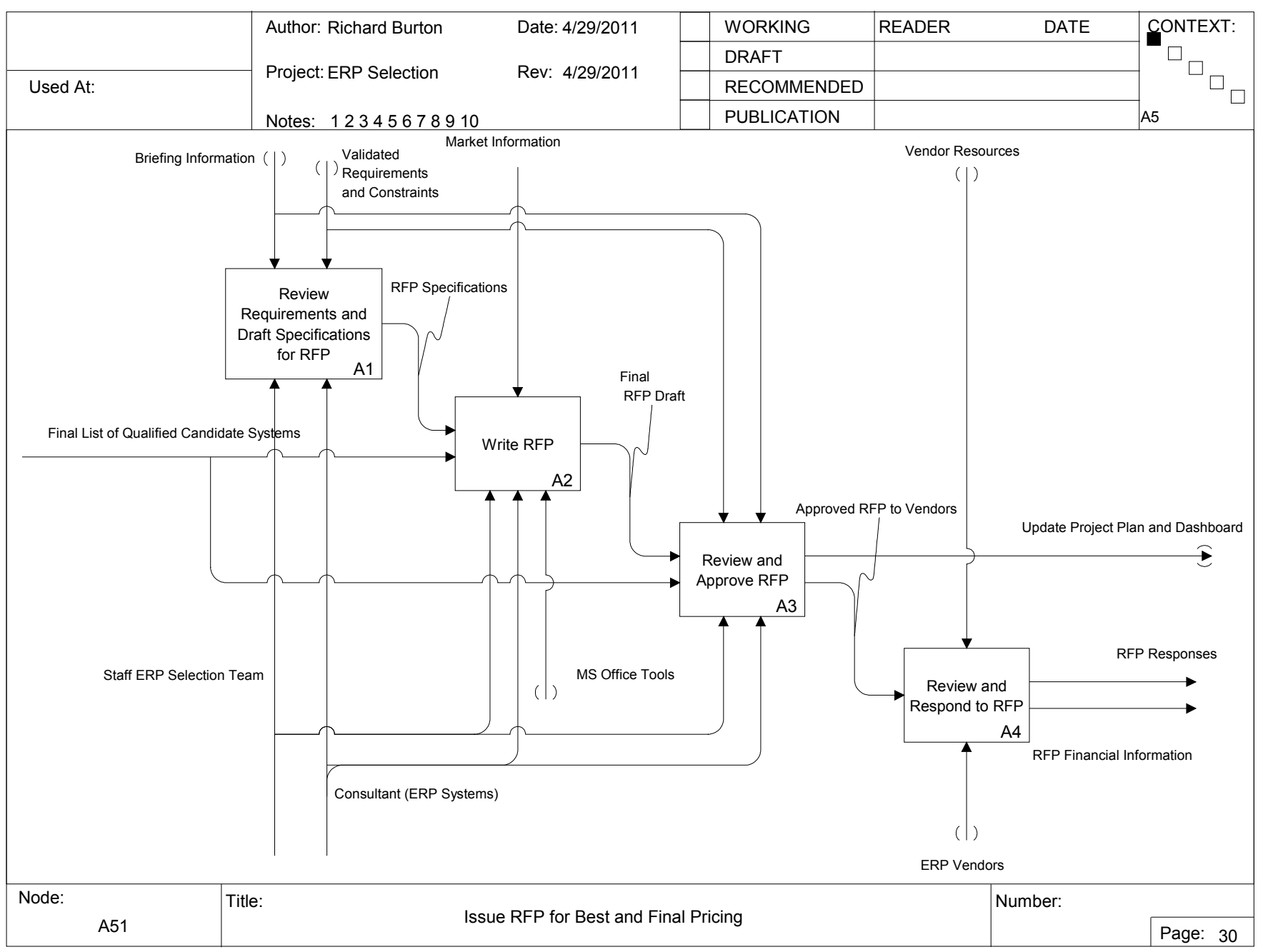

Figure 33 - Diagram A51 Issue RFP for Best and Final Pricing 


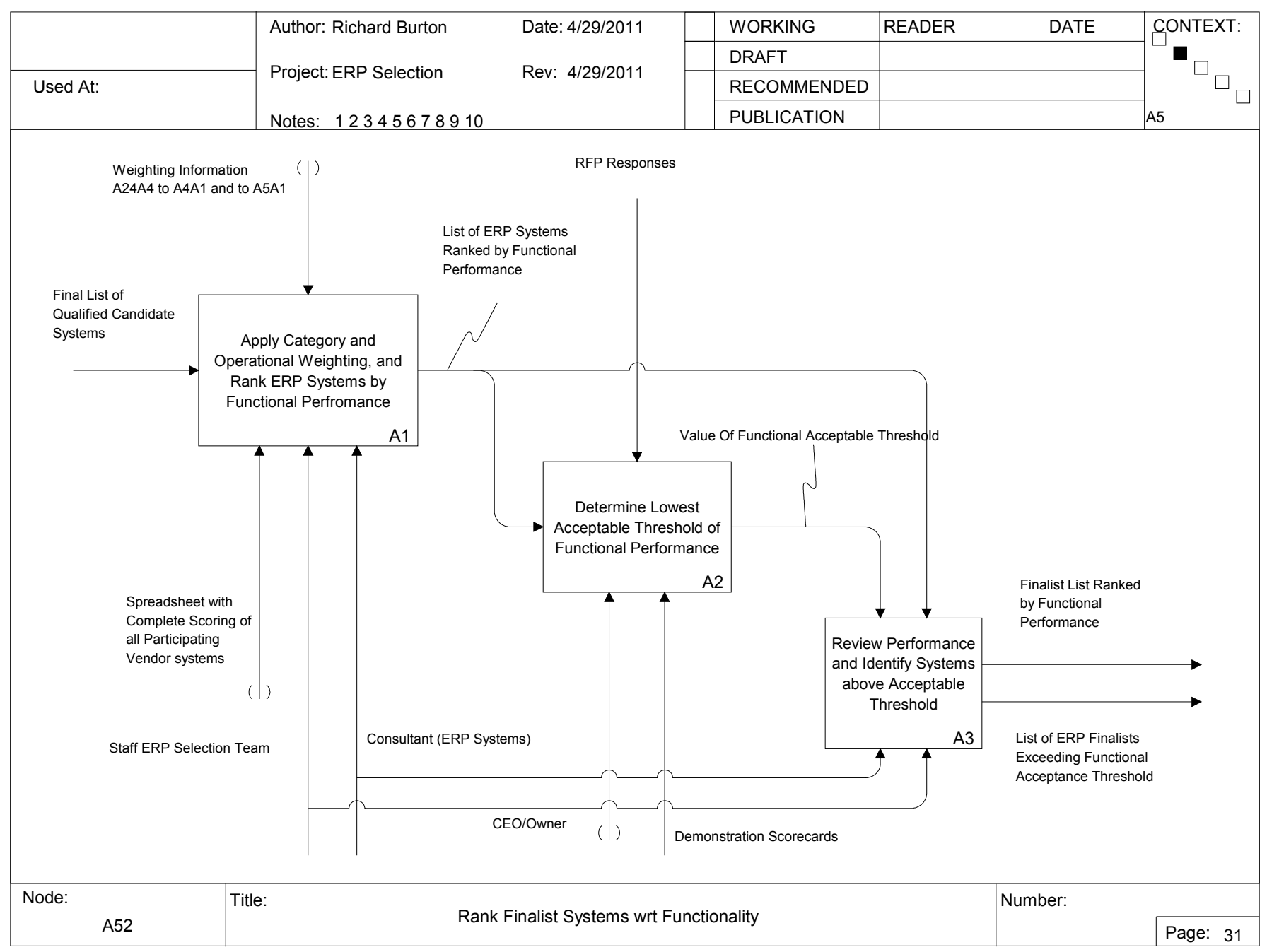

Figure 34 - Diagram A52 Rank Finalist Systems wrt Functionality 


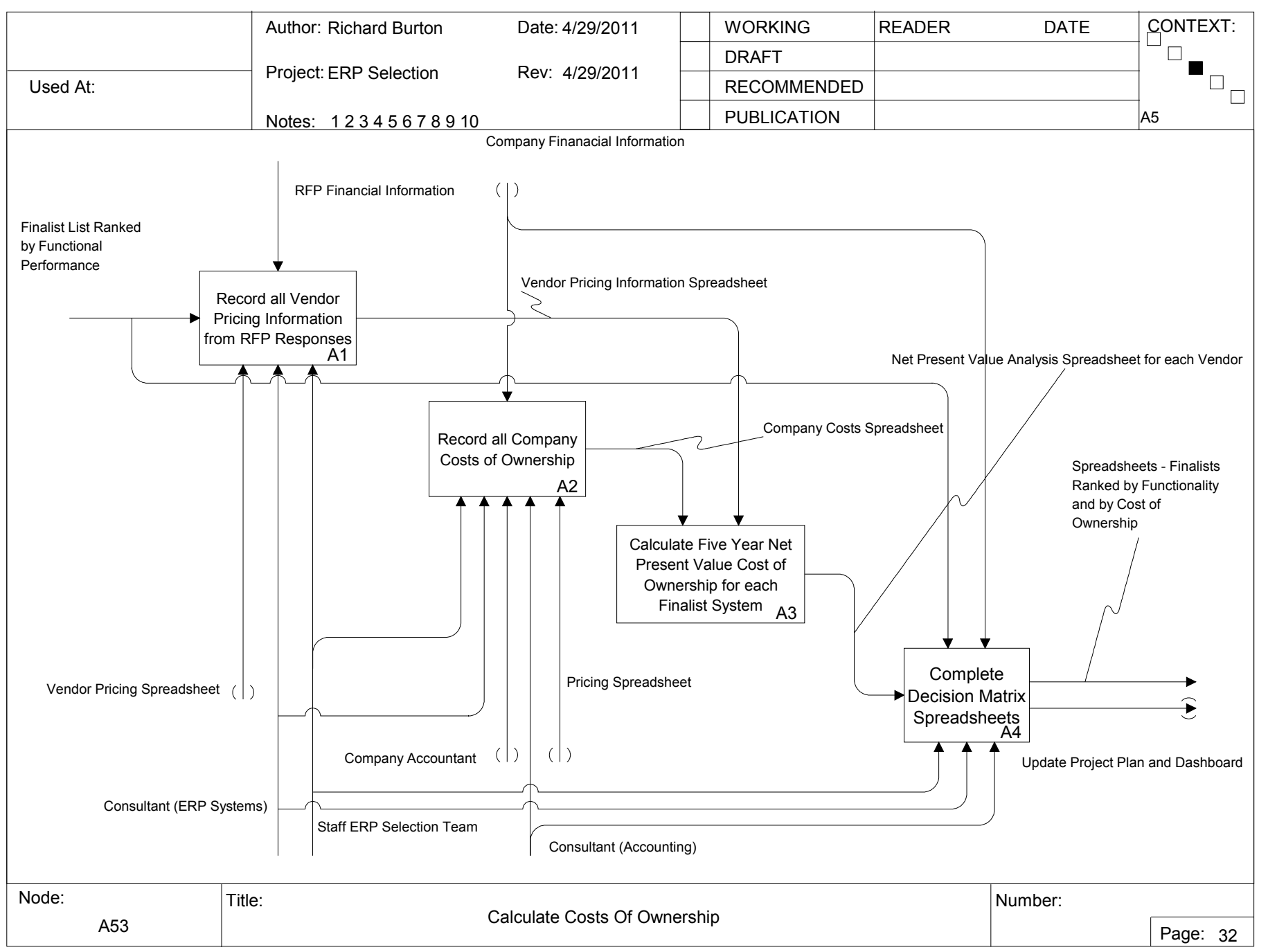

Figure 35 - Diagram A53 Calculate Costs of Ownership 


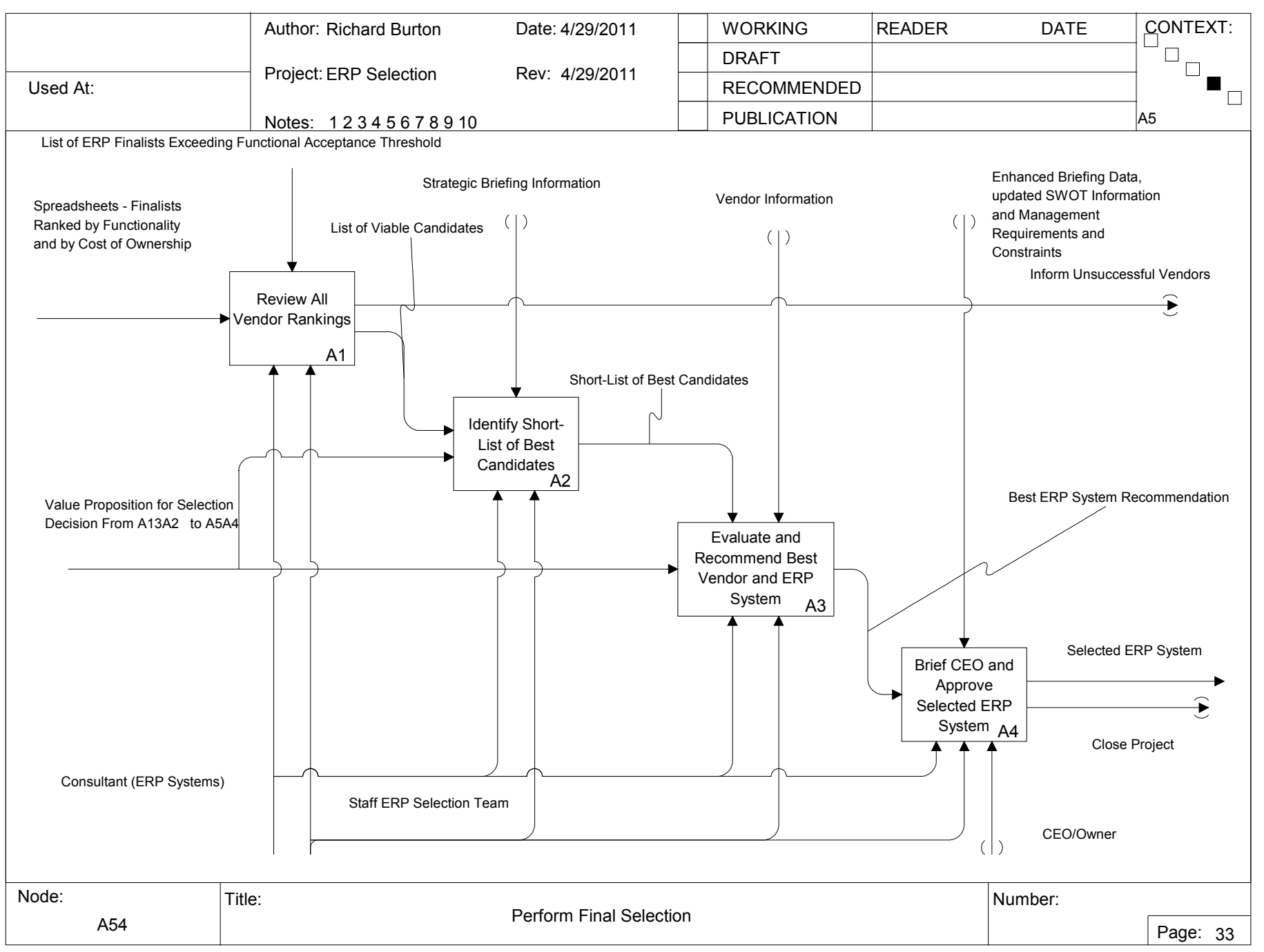

Figure 36 - Diagram A54 Perform Final Selection 


\section{Operationalize the purpose}

Thomann leaves the choice of method for operationalization to the discretion of the methodologist; we have elected to use the straight analysis approach. We use the definition that; operationalization is a process of rigorously defining variables into measureable factors. It is used to define fuzzy concepts and allows them to be evaluated and assessed, both empirically and quantitatively.

Parsing our purpose statement, there are three variables that are to be defined; what do we mean by selection; what constitutes an ERP system and what is a small or medium-sized business?

We define selection as the method which provides a single answer to the question of which ERP system is the best value for a given SME. This is a non-trivial definition since, the selection process could conceivably produce answers in which one or more systems have the same evaluated significance. Consequently, the methodology must include both a valuation scheme to differentiate the systems, and a procedure or method by which ties are broken. We will present an empirical method to compare the requirements to the ERP system capability, and a process to evaluate the level of conformity between the capability and the need. 
An ERP system for our purposes is defined to be a software system, offered for sale or lease which contains; an enterprise-wide set of management tools that balance demand and supply, containing the ability to link customers and suppliers into a complete supply chain, employing proven business processes for decision making, and providing high degrees of cross-functional integration among sales, marketing, manufacturing, operations, logistics, purchasing, finance, new product development, and human resources, thereby enabling people to run their business with high levels of customer service and productivity, and simultaneously lower costs and inventories; and providing the foundation for effective e-commerce. (Wallace, T.F., Kremzar, M.H., 2001)

Finally, we define a small or medium-sized business as a business entity with an employee base of less than five hundred people, and earning revenues of less than $\$ 100$ million. This definition is broad enough to encompass most small and medium-sized businesses without the complication of the categorical restrictions to small business definitions of the Small Business Administration's NAICS based classification system.

VI. Procedural design.

Our analysis of the implications of the purpose provides a good armature to build the initial methodology for the selection of a best value ERP system for a SME. The full draft of the methodology is applicable to and tested by the presented case study, and the lessons learned in that study, along with the responses to the survey are utilized to revise and improve the final version. 


\subsection{Methodology}

The steps that follow are the draft methodology that was applied to the case study instance. The results from the case study allowed us to analyze the results and identify any required adjustments to the draft methodology or its integral methods, and incorporate them in a final methodology.

Step 1: Review company strategy - put the user in touch with the purpose.

We begin by engaging in a comprehensive briefing to provide a formal charge to the company employees and consultants regarding the objectives and outcomes expected in the selection process. Typically this meeting is the starting point for the selection process, and is convened by the company sponsor or a senior manager.

1. All members of the group should be charged with specific roles and responsibilities, there should be a clear articulation by senior managers of the authority of the team, the roles of the employees and consultants, and the scope of their individual span of control with respect to all resources (financial, personnel and other) regarding the selection process. A clear performance timeline and formal outline of the metrics for successful completion should be explained.

2. The limitations (constraints) of the availability of budget, IT resources, and personnel - with respect to the strategic investment commitment for the ERP system acquisition and implementation should be fully articulated. This assumes that a strategic evaluation of these factors has occurred outside the selection process itself, and that resource limits have been established as a part of the company's planning and 
strategic business initiatives. The briefing information will necessarily include an estimation of the gross budget available for a five year period, including the expected costs of IT upgrades, and company and consultant personnel resources for the selection process.

3. The strategic briefing must also define which value proposition is most important to the business; the first proposition identifies which ERP system is the alternative (within a defined budget, and exceeding the minimum functional requirement matching level) that has the least total cost of ownership while gaining as much functionality as possible. i.e. the company will choose the lowest price option, with the understanding that if two or more systems are available at the same price point, they will chose the system with the greater functionality.

The alternative logic to identify a best value option is that the company wants the maximum level of functionality available, (within a defined budget, and exceeding the minimum functional requirement matching level), but that if two or more systems have the same functionality then they will buy the system with the lower total cost of ownership. As these are mutually exclusive logic propositions, one alternative of logic must be chosen.

4. The initial meeting constitutes the beginning of the selection process, and companies should adopt a formal procedure to record the expectations, limitations, roles, responsibilities and authority of the selection team.

5. Typical tools for this step of the methodology are project chartering documents, formal briefing statements, draft communication plans, internal staff assignment letters, and consultation contracts for external experts. 
Step 2: Review of current company processes and process maps to identify essential features and attributes of the existing company business model.

The Selection team leader convenes a meeting(s) to review, and capture requirements and constraints identified in initial meeting, and to assess the processes and procedures currently employed by the company in its operational activities.

a. Participants include all selection team members and consultants. The objective of this event is to establish a common understanding of the company's procedural framework, and to ensure that process mapping fully reflects the company's activities. In addition, the meeting serves to consolidate the data regarding; requirements, constraints, strengths, weaknesses, opportunities and threats (SWOT) information that face the company, and are relevant to understanding and informing the selection process.

b. Team engages in process mapping of any process indicated as absent by the strategic review or that will be required based on expert (consultant) input.

c. Tools used:

i. Access to company process map directory/library or equivalent.

ii. Igrafx ${ }^{\circledR}$ or similar software system.

iii. Spreadsheet/data base tools to be used for gathering requirements, constraints, SWOT information etc.

iv. Any tool selected by users to accomplish the task of understanding the company's process environment. 
Step 3: Determine the functional requirements of the company through focused meetings and interviews.

The Selection team leader identifies and schedules team interviews with top divisional managers to explore their view of company requirements; constraints etc., and gather managerial input. In order to gain organizational buy-in the team should use these interviews to solicit the nomination of key user employees within the manager's department to participate in requirements gathering session(s). these meetings should also include information gathering regarding the broad based assessment of employee capabilities with respect to ERP utilization; determining which employees are familiar with ERP system use, and at what level. The purpose of this information is to consolidate an initial understanding of the personnel training requirements $-\mathrm{a}$ cost factor in later analyses.

Tools used: Formal interview briefing document, outlining objective and intent of the meeting. The team members participating in the interviews should follow a passive information gathering approach, avoiding 'leading' questions - and allowing the manager to volunteer information with minimal prompting, and no commentary from the interviewer.

Step 4: The selection team leader schedules a Joint Requirements Planning session(s). The team leader sponsors a formal joint requirement planning session(s) with managers, key personnel identified in step 3.1, the selection team and consultants. A formal charge to the meeting is given by the company executive sponsor. The team leader nominates a consultant or external facilitator to act as the meeting coordinator, two selection team 
members to act as scribes, and the team leader ensures that the ground rules of the meeting are clearly articulated and enforced. The objective of the meeting(s) is to gather and record all the functional requirements, constraints, and SWOT information that are considered to be important to the company that are evident to the management and user community.

a. Tools used: CASE tool, process mapping tools (Igrafx ${ }^{\circledR}$ or similar), use case tools, word processor, spreadsheet, illustration tools, flipcharts etc.

Reference: Follow a formal procedural pattern such as that provided by Whitten, Bentley and Dittman (2001) or similar.

b. Selection team and consultants meet to validate and quality check the requirements and information identified in JRP session.

Step 5: Determine known current process deficiencies, process map them in an acceptable form, identify features and attributes associated with them.

1. Selection team and consultants meet to identify any known process deficiencies or implied deficiency's revealed in the interviews and JRP session(s).

2. Team leader assigns team members and/or company operational employees to process map new procedures identified as missing or inadequate, and to develop requirements to reflect the new processes.

3. Selection team and consultants meet to validate and quality check the new requirements, and record them with existing list of requirements, and information databases. 
Step 6: Consolidate and review the constraints identified in steps 1-5.

1. Selection team and consultants meets to review, quality check, validate and record all relevant system constraints.

2. Tools used: Spreadsheet list of constraints.

Note: Steps 5 \& 6 may be combined meetings, at the user's discretion, although for most small businesses the resources employed in these activities will probably be the same for each step, so must be sequentially executed.

Step 7: Review the company's Information Technology Baseline.

1. A formal interview with the chief technology officer or equivalent should be convened, with the objective of understanding the status of the IT hardware and software systems employed by the company. The objective of this step is establish the baseline IT capability and employee competence level, employee familiarity with ERP systems from an IT integration perspective, associated training requirements, and hardware configurations and accessibility issues.

2. The information regarding the company hardware, network and software capability baseline should be captured for use in later assessment steps. Tools used are spreadsheet or database software to consolidate and record the information.

Step 8: The selection team identifies all available ERP systems in the marketplace.

1. The identification of all ERP systems available in the marketplace may be accomplished by any one of the following methods:

a. Aggregate lists from online resources, some possible resources are: 
Capterra.com offers an extended list of commercial ERP software solutions, vendors pay a fee to be listed and higher fees to be showcased. The current Capterra list includes two hundred and seventy nine separate systems. The list simply presents the commercial advertising synopsis of the system and does not classify nor differentiate the service or market sector applicability of the software. No cost or performance information is available for the ERP systems that are included. Softselect.com offers an online data resource of ERP vendors, in an annual survey produced by APICS - the Association for Operations Management. The list is unique in that it provides a classification of each ERP system which relates it to its target industry segment. No cost or performance information is available for the ERP systems that are included.

b. Any other information aggregation method which allows the identity and outline capability of the software to be verified with a vendor. Such lists are typically kept and provided by the expert consulting entities that are employed to provide expert assistance to the company selecting the ERP system.

2. Tools used spreadsheet or database tool to collect and consolidate the information regarding available ERP systems. This list is the starting list or long-list of candidate ERP systems.

Step 9: The selection team must obtain the relevant information from the list or by information requests (RFI) to the vendors to determine the industrial sector applicability and preliminary cost information for each system.

1. The selection team and consultants meet to review the long list obtained in step 6 which identifies each ERP system, and the obtained information. 
2. The selection team and consultants analyze each candidate system with respect to the known cost constraints, and the known data to select those systems which have application to the company's industrial sector, and which have cost structures that are less than the five year budget identified in step1.

3. The selection team and consultants resolve the long-list into a short list of candidate systems which are viable candidates based on preliminary cost information and industrial sector applicability.

4. The selection team accumulates the RFI responses from the short-listed candidates and begins to gather any available information regarding the normal business practices of the candidate vendor companies. The team should use Dun and Bradstreet business information, BBB information and any information available from the State in which the potential vendor is incorporated. The team should include a request in the RFI to the short-listed vendor companies to identify independent references for their services, which should be consulted for verification purposes. An interview question matrix, to be used for all references, identifying the key concerns and business relevant issues should be developed using the constraints and requirements information in hand.

5. A team review process is conducted to evaluate and determine if any of the vendors are responsive with respect to the constraints, and the short-list reduced to include only the qualified candidates.

Step 10: Organize requirements by category, and analyze them 
1. The selection team and consultants utilize process mapping tools, to decompose requirements into functional descriptions of processes and to identify the underlying attributes and activities of each function. Each function is categorized into operational categories; finance, (AP, AR, General Ledger, etc.), operations (manufacturing, design, quality, CRM, etc.) and each function is to be listed within its respective category.

2. For each requirement, the selection team and consultants determine who performs or should perform each activity.
a. Analyze the workload changes
b. Assess personnel training requirements
c. Assess changes in staffing requirements for the standard volume of transactions described in step 1.

3. List functions from requirements in their respective category, list the activities within the function

4. Selection team obtains senior management input on the relative importance of each category. Category weighting is normalized.

5. Selection team obtains assessment of operational managers of the absolute importance of each activity with respect to a numerical scale from 1 to 10 .

Step 11: Conduct a benchmarking survey, of similar SME business entities to determine performance criteria, inform the weighting process and assist with company specific procedural design. 
Since, the information derived from the survey instrument is intended to inform both the weighting process for the technical requirements, and to ensure that the selected ERP system process is benchmarked against similar business enterprises, the following procedures should be adopted.

a. Define the survey objective to be to inform both the weighting process for the technical requirements, and to ensure that the selected ERP system process is benchmarked against similar business enterprises.

b. Identify the target audience - which companies are competitive and sufficiently similar to our business model and have selected and deployed an ERP system recently?

c. Identify who at the company is our target respondent? $\mathrm{CEO}, \mathrm{COO}$, Director?

d. Determine how to conduct the survey - mail, email, interviews etc. Interviews are regarded as the most effective mechanism, but have a high resource requirement from both the interviewer and interviewee. Structured interviews using passive questioning techniques are preferred.

e. Ensure sample is relevant to the objective - qualify by same business sector, similar (revenue, product, service, personnel headcount etc.).

f. Use adjectival rankings with fixed quantitative Likert type scale.

g. Keep total required response time to less than one hour.

h. Assure anonymity of respondent's identity and company.

Step 12: The selection team and consultants develop a performance test scenario which consists of demonstrable standardized processes required from the successful ERP 
system. In addition the team produces a performance scoring matrix document for each requirement and its activities.

1. The performance review scenario's should test, at the least, the candidate ERP systems ability to perform with respect to all of the category's of transaction identified in step 10. In addition, the scenario's should avoid test situations that test only one transactional category i.e. perform an AR transaction, but should rather test the integrated performance of the ERP system as a whole. Such a scenario might include showing the purchase of a component for a manufactured item, e.g. intrinsically testing the purchase order initiation, receiving, quality, AR/AP and warehousing (inventory) functions of the ERP system.

2. The scenarios should be reviewed prior to release to the candidate vendors and each vendor should be asked to respond to the same performance test and evaluated using the same evaluators.

Step 13: The selection team leader schedules appointments with short-listed candidate ERP vendors to demonstrate the ERP system under the fixed scenarios.

a. The selection team develops a scoring matrix tool, typically a spreadsheet matrix, containing the listed requirements, by category, from step 10 . The scoring matrix will constrain the raw evaluation scores for each vendor to a chosen scale i.e. zero for completely non-responsive to the requirement, five for the best possible response.

b. The matrix should not show categorical or requirement specific weighting to the evaluator, to avoid bias. 
c. A descriptive guide should be provided to help the evaluator understand the evaluation scale i.e. direct a linear response in which the responsiveness between scale integers is equal.

d. Require integer scale responses.

Step 14: The selection team, consultants and nominated users review the scenario demonstration for each system. Each member completes the scoring matrix for each system and each requirement. The aggregated score for each ERP system becomes its functional raw score.

1. Presentations are scheduled - no more than one per day.

2. Team and evaluators use consistent setting - i.e. same conference room.

3. Presentations are limited to a fixed duration.

Step 15: The selection team calculates the weighted value of each system's functional matching score, by multiplying the raw functional score by its category weight determined in Step 10.4, and the activity weight obtained in Step 10.5. We calculate the hypothetical perfect score; representing $100 \%$ of the functional requirements are met, and calculate the percentage of that functionality that is achieved by each system. The functionality for each system is recorded as its percentage of functionality.

Step 16: The selection team and consultants identify the final (five) ERP systems with the highest weighted functional raw score. 
Management is requested to review and identify the minimum acceptable level of functional score (i.e. identify the redline value below which the ERP system is determined to be non-compliant with the requirement to capability match). Noncompliant vendor ERP systems are removed from consideration.

Step 17: The selection team obtains the best and final cost information by RFI/RFP to the final list vendors.

At a minimum, each vendor should provide the following data; cost of acquisition, cost of maintenance or lease per year for five years, cost of implementation support for first year, cost of customization for mismatched functional needs.

Step 18: The selection team and consultants determine the five year costs internal to the company.

The budget assigned in step 1 is considered to be a hard constraint.

a. Using the analysis of earlier data collection activities, and interviews, the selection team leader coordinates staff in evaluating the following internal costs; cost of additional workloads, cost of consulting support, cost of training, and cost of IT upgrades.

b. The items above are a minimal list of potential internal costs; the company employing the methodology should collect all costs which may affect the total cost of ownership over a five year period that can be determined from their accounting information. 
Step 19: All costs are distributed over a five year time horizon and the NPV total cost of ownership of each system is calculated.

Step 20: The costs determined in step 16 are deducted from the available budget determined in step 1. The selection team and consultants determine if any system has now become unaffordable due to the reduction in the total budget, and eliminate it from consideration.

Step 21: We now have a qualified short-list of alternative ERP systems which are at least minimally responsive to the functionality requirement, and have costs within our budget. We apply the selection logic mandated in step 1.

a. If we desire the lowest cost alternative, we rank the systems from lowest cost to highest, and pick the least cost system. If the total cost of ownership for the lowest cost of ownership alternative(s) is a tie between two or more systems, the system with the highest functional percentage is chosen. If there is still a tie we review which system has the best score in the highest rated category of requirements, and pick that system. If there is still a tie we apply the choice at the next category in sequence until the tie is broken.

b. If we desire the alternative with the best functionality, we rank the systems from highest weighted functional percentage to the lowest, and pick the highest functionality system. If the functional score is a tie between two or more systems, the system with the lowest total cost of ownership is chosen. If there is still a tie we review which system has the best score in the highest rated category of requirements, 
and pick that system. If there is still a tie we apply the choice at the next category in sequence until the tie is broken.

Step 22: The methodology should be tested and reviewed to determine if any changes or improvements can be accomplished. 


\section{TEST AND VALIDATION}

\subsection{Case Study}

A case study was conducted, employing the draft methodology developed in Chapter IV. The ABC Company (a pseudonym used by agreement with respect to their desire for anonymity) is a custom engineering manufacturer of defense related systems. It is a low volume/high mix manufacturer, performing design, development, and system integration for custom engineered systems. In addition to its manufacturing capability, the company has programs which perform Research and Development (R\&D) as a client service, and a small engineering resource capability which is used for Internal R\&D (IRAD), and competitive research work such as SBIR/STTR from client agencies.

Step 1: The ERP system selection process was initiated based upon the company's senior management's recognition of severe strategic information flow limitations, and operational constraints that were affecting overall efficiency. An internal team of senior managers, with responsibility for the systems implementation process was assigned by the ABC management team.

When viewed from a process engineering perspective, the selection of an appropriate ERP system and its eventual successful implementation is a change management event within the context of a multidimensional process change management framework, as proposed by Al-Mashari (Al-Mashari M. , 2002): 


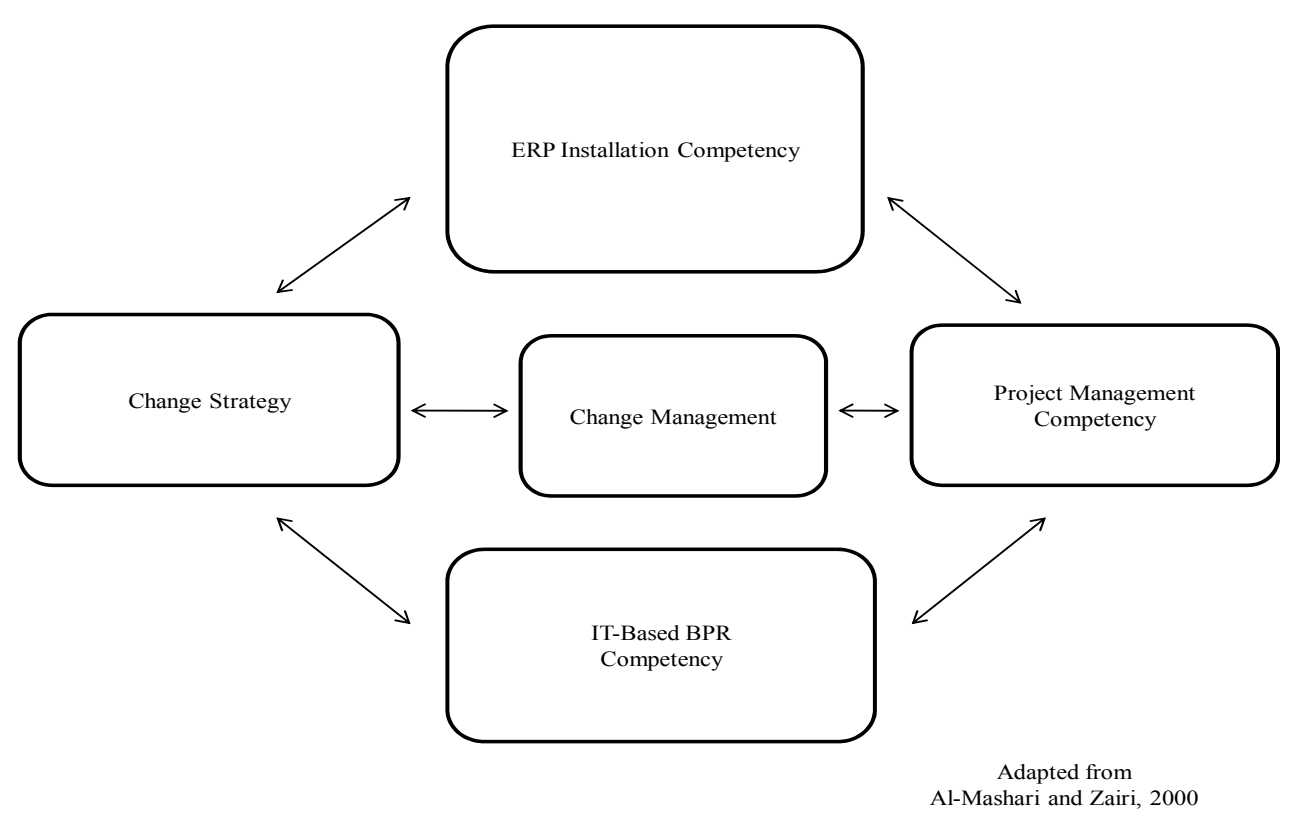

Figure 37 - Core Competencies for ERP adoption.

The primary executive decision to proceed with an ERP system purchase is the responsibility of the senior management team and owners of the company. In this case the managers and owners had reviewed the options and decided that the only viable choice for $\mathrm{ABC}$ was to buy a commercial-off-the-shelf (COTS) ERP system. Accordingly, a wide ranging review of the strategic intent, for the acquisition of the ERP system, and delineation of the expected performance outcomes were elicited from the company executive ranks. This process was adopted to fully understand the risk to reward expectations of the ERP implementation process within the management group, and to ensure that the correct context was adopted to review resource allocation issues throughout the selection and implementation processes. The key points of discussion 
contributing to the decision space for the team were; determining the business objectives of the project, articulating the risks involved by committing to the project and understanding the nature of the changes involved in the effort. This process was adopted to achieve the highest degree of trust and to set a baseline for the communication strategy to be adopted by the consulting team and the senior management group. In their review of the change strategies for effective IT project implementation, Kuruppaurachchi et al., suggest a model which highlights the multiple inputs and continuous nature of the high level review and management intervention that is required to successfully implement an ERP project. (Kuruppuarachchi, 2002)

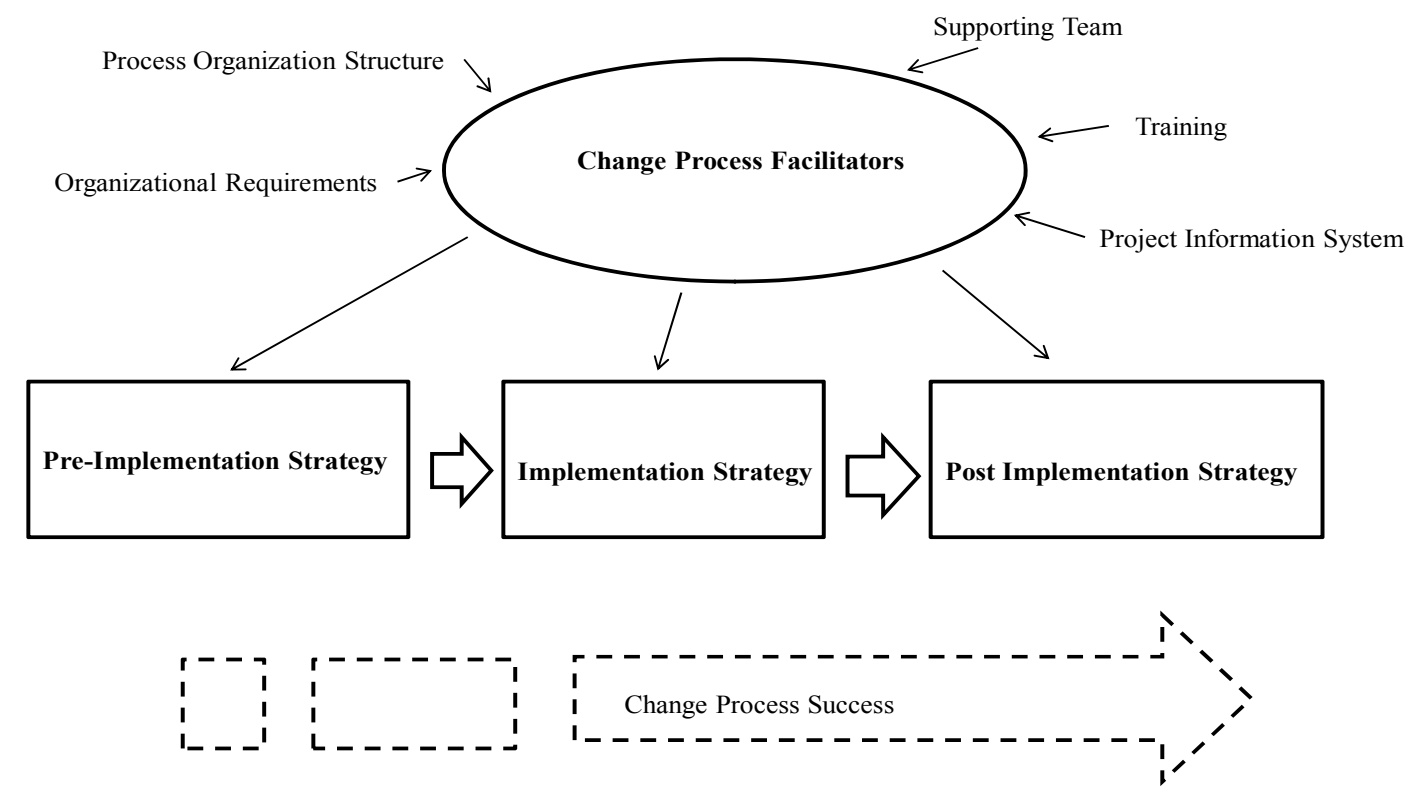

Source: Kuruppuarachchi et al., 2002

Figure 38 - Strategic Considerations for Change Management

The senior management briefing resulted in the definition of the process organization structure, with the nomination of the organizational advocate and project lead. In 
addition the FIU and NGC (a pseudonym) group members were formally identified as the support consulting resources, responsible for coordinating the selection process, identifying and facilitating the organizational training needs, and establishing and maintaining the project information system resources. A formal project reporting and communication structure was defined with the ability of all key participants to share essential information through a web-office scenario; in addition a reporting schedule and process timeline was established. Formal selection process team communication was established between both the $\mathrm{ABC}$ team leader and the NGC team leader, with informational linkages to the $\mathrm{ABC} \mathrm{CEO}$ for periodic updates and progress reporting.

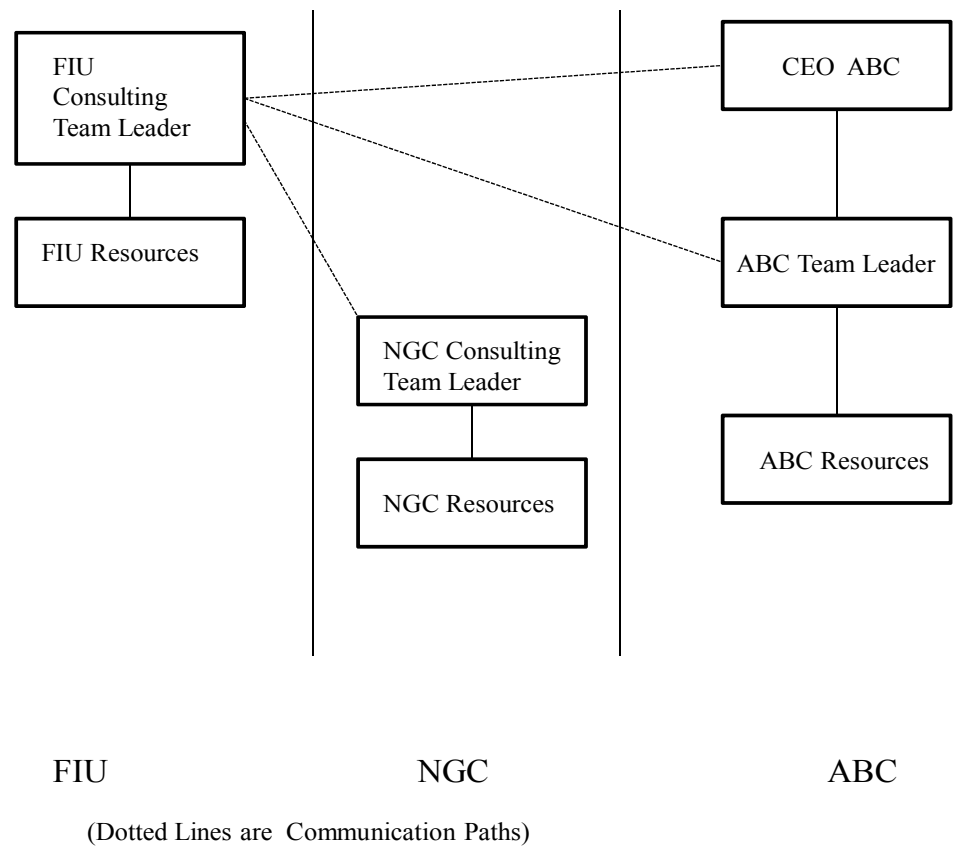

Figure 39 - ERP Selection Team and Communication Paths 
The team make-up for the selection process consisted of staff from FIU, a member of $\mathrm{NGC}$ as a consulting partner, and a senior manager form $\mathrm{ABC}$ as the communication and decision conduit to $\mathrm{ABC}$.

Finally, the senior management team agreed to make personnel available to begin a comprehensive, organizational requirements gathering process.

Senior management identified the priority and the primary objectives for implementing the ERP system at $\mathrm{ABC}$ as; to obtain accounting and financial capability improvement, with reliable and consistent reporting, and better financial analysis capabilities; gain integrated manufacturing functional efficiency improvements, with full logistical tracking throughout the company.

They also provided a more specific outline of the company and its current business posture. $\mathrm{ABC}$ has grown steadily since its initial establishment to become an efficient and well managed supplier to DOD and client companies. Despite an unsatisfactory early experience with first generation ERP systems, and a failed implementation, the company exhibited a clear need to realign its accounting software to a more sophisticated system. The managers urged that, the selection process for this system should focus on identifying a system meeting the primary need to provide reliable financial reports and reduce the data entry and workloads of the accounting group. In addition, but with lower priority, the company wanted to take advantage of the ability of an ERP system to couple with the manufacturing, logistics and material handling requirements of the company. 
The general system requirements identified for the ERP system as a whole were stated as; the system should be suitable for the current organizations financial systems with revenues of $\sim \$ 15 \mathrm{M}$, and should be scalable to accommodate growth beyond the projected $\$ 50 \mathrm{M}$ organization. At the then current workload, the organizations financial systems supported 100 to 250 employees and it was specified that the ERP system should be able to support more than 15 concurrent users at all times, with an estimated peak load of 30 users. The budget was set at $\$ 500,000.00$ for a five year period.

$\mathrm{ABC}$ expressed a preference for a locally hosted system; they agreed that if an appropriate cost/benefit analysis suggested an alternative web based system, it should be considered. $\mathrm{ABC}$ also had an institutional preference for 'open architecture' systems, and they stated that they would like to have access to the source code of the selected system. Senior management at $\mathrm{ABC}$ facilitated a requirements gathering process which allowed access to several levels of employees in the accounting, logistics, manufacturing and engineering units within the company, and they participated in both open and closed door sessions to align the expectations with the strategic objectives of the project. The senior management briefing process was the chartering phase of this implementation project, and at its conclusion the team had been nominated, resources allocated and the management team had been fully apprised of the risk potential of the entire process.

The CEO of the company was asked to identify the primary selection logic, which was expressed as a desire to buy as much functional capability as possible, within the budget, and assuming that the capability exceeded a minimum threshold level of $66 \%$. 
Step 2: In order to complete the teams understanding about $\mathrm{ABC}$ 's current business practices, several working meetings were dedicated to reviewing the company's processes, and we were provided access to the business process and procedures manual, which outlined the various company organizational units, and their standards and procedures for their functional responsibilities. The team worked with $\mathrm{ABC}$ employees to review system requirements and specifications for business procedures, and assess the current technical infrastructure. New process maps were developed for several processes that were not fully mapped in the original documentation. The overall business process map was revised, using Igrafx software.

Step 3: The selection team was afforded the opportunity to interview the key departmental managers at $\mathrm{ABC}$, including; the $\mathrm{CEO}, \mathrm{COO}$ and the directors of finance, manufacturing, quality, and engineering. Several key requirements were identified, and recorded in these meetings. See example interview notes in Appendix

Step 4: $\mathrm{ABC}$ and the consulting team initiated a requirements gathering protocol which began with a Joint Requirements Planning session over a period of four days. All aspects of the business were evaluated and over 25 individual employees were consulted with in ad-hoc and structured interviews. The structured interviews, with multiple participants were most valuable in refining the corporate team knowledge base of the then current operating limitations and the perceived sources of inefficiency or areas requiring improved systematic approaches. 
Business and manufacturing functions were currently served by support service software with varying degrees of integrated capability. The entire suite of software provided a loosely integrated overall business system for $\mathrm{ABC}$; however the main deficits and weaknesses of the then current capability were clearly demonstrated in the client's dissatisfaction with the overall utility of the system.

At the time of the requirements gathering meeting $\mathrm{ABC}$ was operating with a number of business critical legacy software systems, which they hoped to supersede with the introduction of the ERP system. For primary accounting functions $\mathrm{ABC}$ was employing the commercial software system Peach Tree accounting. This system is a relatively low cost software system with limited capabilities, designed, developed and maintained by SAGE North America. The system is a part of a series of software solutions that include HR, accounting, CRM and ERP components, although $\mathrm{ABC}$ had not elected to integrate any of the higher order functions. Most other documents (including BOM, traveler, quotes, part drawings, etc.) were stored and viewed using an in-house developed system called EZ MRP. EZ MRP is a Microsoft Access based system that runs on the network at $\mathrm{ABC}$, enabling users to share the data. Significant $\mathrm{ABC}$ time had been spent in developing individual customized format reports and much additional time was spent to customize data input forms. Engineering drawings and drafting software were run on client computers, and the data stored on the network. This system provided limited support for manufacturing, logistics and was not integrated with any accounting 
functions. ABC was using ADP-EZ Payroll and ADP - EZ Labor, which were perceived to be providing adequate payroll and labor hour tracking, in fact, $\mathrm{ABC}$ managers indicated a desire to retain or integrate this software system into its final solution if possible. Other software systems included; PULSE which was a locally developed graphical reporting capability for EZ MRP data, ACT a commercial contact management and business development tool, MSEXCEL ${ }^{\circledR}$ used for custom reporting and data processing/manipulation, and TIMEBANK, a middleware to integrate data between ADP and Peachtree. For the full suite of software currently running at $\mathrm{ABC}$ see Appendix C. The company's entire business data was backed up periodically - usually, weekly. Information technology employees indicated that the data backup cycle took between four and five days to achieve full back-up.

A number of features and attributes were identified as favorable characteristics; $\mathrm{ABC}$ management were pleased that the software systems were internally managed, and that the then current system could be modified by local IT personnel or consultants, to add specific client needs and functional enhancements. The system in use had good compatibility with the Microsoft Office ${ }^{\circledR}$ Suite of tools, especially Excel and Access, allowing manipulation of data to produce custom reports. ABC like many SME's is highly cost conscious, and the fact that the existing systems were locally hosted, and had no third party hosting fees was regarded as a positive attribute. In the same vein, the staff's high familiarity with the software was regarded as an important advantage, again reducing costs by having a low training requirement. Equally, the overall system 
maintenance costs were very low, and most of the software conformed to the company preference for open architecture software.

Over the last five years of operational experience, $\mathrm{ABC}$ had made modest upgrades and changes to their software systems environment, but the drive to adopt a new ERP system was driven by an awareness of major concerns and weaknesses in their systems. The inhouse system(s) were highly labor intensive; particularly with respect to data entry, and report development and creation. The distributed nature of the software systems meant that there were multiple sources of data input which undermined the consistency of strategic and management level reports. Reports generated by the local systems were frequently at odds, mostly attributed to the fact that data from different systems were producing conflicting results. An example of the data inconsistency was the fact that Peachtree captured the dollar value of inventory but not the quantity on hand, while EZ MRP captured quantity but not dollar amount. Simple logistical information; quantities on-hand, on order etc., did not conform to the corresponding accounting system information. Manufacturing details, and design information were poorly handled; BOM's were maintained as spreadsheets, there was no clarity on the R\&D acquisition of material, and a need was identified to include engineering $R \& D$ stock use into the overall logistical reporting process. Inventory management of items segregated on receiving or rejected in manufacture was poor, items that were indicated as in inventory were frequently missing, or unaccounted for in quarantine. Serial number tracking of inventory was not accommodated, and EZ MRP did not display the current inventory detail in the general ledger. Manual adjustments, and field verification was frequently necessary. EZ 
MRP had no ability to attach diagrams/schematics, drawing, ECO's etc., and did not track work in progress (WIP). EZ MRP's reports provided conflicting data, undermining confidence in the veracity of the information, and the software development and updating costs were not accommodated in the future business model. Software field space and other trivial software issues were making data entry and recovery increasingly difficult. Administrative functions supported by the legacy systems also contained data inconsistencies or failed to accommodate standard business information needs; standard terms \& conditions, and contract details had to be entered separately in each instance. Other integration issues related to HR functions, for instance; ADP did not allow applicant tracking or extended hiring information functions, and Peachtree did not receive information from ADP until Peachtree closed i.e. no real time coordination of information.

Step 5 \& 6: No additional process deficiencies, beyond those identified in step 4 were identified. The team met to discuss and validate the requirements, constraints and to review the SWOT analysis information gathered during the interview process and subsequent JRP sessions. Qualified requirements and constraints were added to the spreadsheet. A comprehensive list of the initial requirements for $\mathrm{ABC}$ is attached as Appendix B.

Step 7: An assessment of $\mathrm{ABC}$ 's technology infrastructure was also conducted and the background information gathered for the technical feasibility analysis was acquired through interviews with $\mathrm{ABC}$ personnel while during the site visit to determine the 
current IT infrastructure at $\mathrm{ABC}$. The information was used to define the existing IT Infrastructure of $\mathrm{ABC}$ in order to define a baseline for each recommended product's technical feasibility within the current infrastructure. ABC uses a Microsoft Windows network environment operating under an NT domain. The network architecture was based upon a standard TCP/IP connection commonly found in most networked environments. A hardware and software firewall (embedded in the router) was implemented but we were unable to define to what level of security and how well the firewall was maintained. $\mathrm{ABC}$ connects to the internet through a cable broadband connection which is shared across the network (LAN).

ABC's information resources are centralized around 12 PC acting as servers; handling engineering task and other applications. There are also three main servers. The tasks of these three servers were; Primary Domain Controller, Secondary Domain Controller, and a File Server. The file server provided data storage to the entire user community at $\mathrm{ABC}$ (at the time of the review the total storage capacity of that server was unknown, but later proved to be inadequate for ERP use.). The detailed configuration map of the server system is seen in Figure 4. The IT staff group was charged with acquiring and configuring a new server to replace the current file server. 


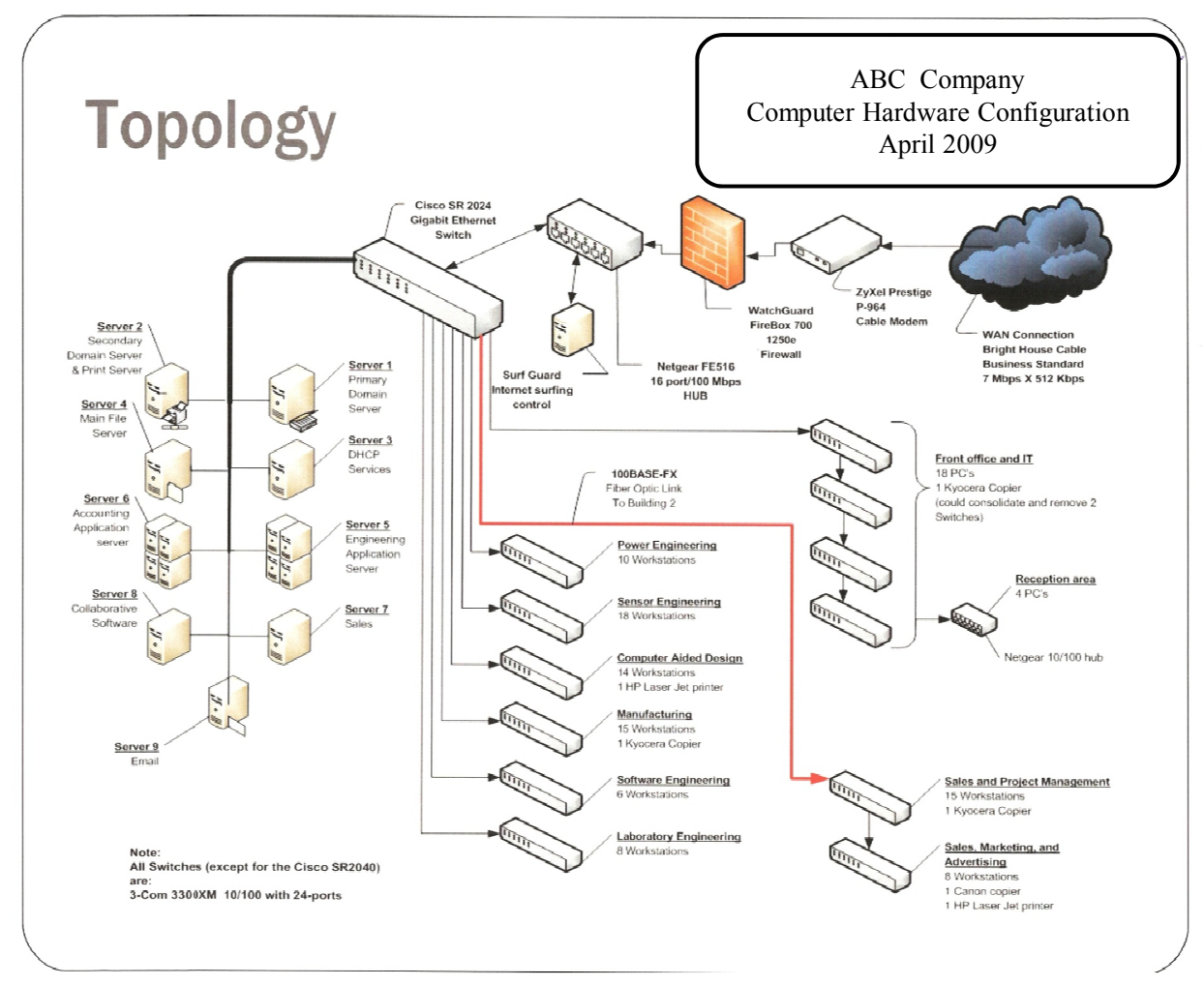

Figure 40 - Technology Baseline (Network Hardware) 2009

At the time of the review there were two full time personnel to manage IT needs. The IT group's span of responsibility extended to maintenance and operations of all IT assets. These included, the servers mentioned above in addition to 60 desktop client machines and several network printers. Current IT staff levels and training were regarded as adequate and their credentials and experience indicated that they were capable of maintenance and operational support of the existing and future system requirements for an ERP system. Although, from a productivity perspective, the IT personnel had no spare time, and there were no clear lines of reporting to senior management, they were keeping up with the operational and maintenance load. It was clear that there was no additional or substitute resource for situations such as vacation coverage and sickness 
coverage. In addition, certain system upgrades which had been long standing needs within the IT infrastructure had been "shelved", until such time as the staff would have spare time to begin these projects.

It was clear that the natural growth processes of $\mathrm{ABC}$ 's IT infrastructure had been accommodated in a professional and adequate manner, however the ad-hoc decision making and somewhat random approach to IT services had led to a situation where some degree of rationalization of the system environment was clearly needed. In addition, the IT staffs were all technicians, and no line management member had sole responsibility for the IT infrastructure. As a consequence there was no strategic plan for IT infrastructure, staffing or operations, and no budgetary controls were in place that were consistent with the defined operational needs. Upgrades were achieved on a ad-hoc basis; for instance, funding for new equipment or extended services was obtained by advocacy by technical staff to line managers with no IT management purview. If the manager was 'convinced' of the need that equipment or software was purchased and the IT staff was asked to integrate the components into the overall system. In view of the need for system hardware upgrades and the impending increase in IT staff time needs, associated with the ERP implementation; it was recommended to make organizational adjustments to develop an IT system strategy. In addition, the nomination of a formal IT manager, with authority to design and coordinate the IT strategy and to manage the budget and other resources for the entire system was proposed. 
Initial review reports and recommendations were developed after a thorough analysis of these requirements. It was clear that $\mathrm{ABC}$ had outgrown EZ MRP and the software suite being employed was showing signs of approaching their limits, and that the company was in need of a complete ERP System to improve control of their business processes. While $\mathrm{ABC}$ viewed its accounting practices as functionally acceptable and adequate, it was apparent that there were opportunities for the company to achieve better efficiency in the short term. It was also clear that $\mathrm{ABC}$ would benefit from the scalable quality of an ERP system, which would allow efficiency improvements to be found in the short term, and for those improvements to propagate into its business administration and management as the company grows. In addition, the integrated nature of an ERP/MRP system would provide additional benefits, such as addressing $\mathrm{ABC}$ 's need for a system to integrate both accounting and manufacturing activities.

$\mathrm{ABC}$ staff was clearly well trained, they demonstrated to be sophisticated users of their original software systems, and the corporate character was open to the adoption of the 'right' ERP system, perceiving it as a real benefit to their work tasks. This feature of the company mitigated one of the key failure modes often encountered in ERP selection and implementation - that of internal resistance to change, at the staff level. Beside the clear need for an ERP, and the precise understanding of the needs addressed by the staff and management, it was also apparent that the introduction of an ERP system would be made much more effective by upgrading the existing server/network environment, before or in parallel to the introduction of the selected ERP system. As a minimum requirement, the host server of the ERP system, critical to having an appropriate development and 
operational environment, needed to be upgraded. The server/network capability improvements uniquely focused on those that would meet the minimum performance standards for a new ERP system. While peripherally related to the ERP implementation process, it was noted that the IT systems back-up process for all operational systems at $\mathrm{ABC}$ took 4 to 5 days. This is a performance standard below industry benchmarks, and it was recommended that the backup process be enhanced globally at $\mathrm{ABC}$. The adjustments of the server/network configuration for the purposes of the ERP were seen as a good time to make the required back-up modifications to secure the company's data.

$\mathrm{ABC}$ needed to select a robust scalable ERP tool with the capability to produce; reliable financial data, consistent reporting, an ability to accurately capture costs and underpin job costing processes, and to account for inventory throughout its operations, with enhanced abilities to include project management, and production tracking.

The initial collection of requirements acted as the starting point for a formal process to identify and purchase the best ERP system for $\mathrm{ABC}$, based on an independent evaluation of the available systems in the marketplace. The characterization of the requirements is a highly non-linear process which in practical terms requires a methodology to navigate the interrelationship of many variables and attributes, and stakeholder opinions.

Step 8: At the time of the assessment, there were more than two hundred commercial software packages available in the marketplace; to sort the systems the team developed 
an assessment matrix which grouped the software systems by industrial sector. (Appendix D)

Based on $\mathrm{ABC}$ 's business interests and product set, four relevant business sectors were nominated as likely to contain candidate systems with a high level of match to the known requirements set. This process was adopted to allow a reduction in effort and costs, with the understanding that the highly specific needs of a small manufacturing business, serving the aerospace and defense sector, has highly specialized software needs which are only likely to fully accommodated by software designed for customers in that business sector or closely affiliated to it. Three team elements (FIU, NGC and ABC) participated in identifying the broad range of available systems, and a recursive process was initiated, consisting of; data searching, evaluation, feedback, adjustment and input with the design deliverable being the candidate short list. It should be noted that throughout the process, new candidate systems were proposed for evaluation and at no time, until the final choice process, was the incorporation into the list of qualified candidate systems closed. The selection process timeline was designed for approximately six months, at the end of which a group of finalists were to be shortlisted for final selection. The selection and evaluation criteria were based upon both the initial requirements and amendments to those requirements that were introduced during the life of the selection process.

Step 9: Overall, the team adopted a three phased selection criteria gating process for each software vendor. In parallel, the team developed a set of Request for Information (RFI) documents, which were prepared for submission to an intermediate group or long-list of 
vendors. The purpose of this document was to compare the vendor's self-assessment of their software offerings against a singular list of attributes and functional requirements that were derived from information obtained in the planning stage.

At the first stage the criteria were related to industrial sector suitability of the candidate systems and whether they possessed the appropriate capability to handle the unique requirements of a small business. At this stage, certain candidate systems dropped out of consideration due their high cost of acquisition and maintenance. Once a "long-list" of vendors had been qualified, the RFI was sent out to each of them to elicit the selfevaluation, and to derive primary cost information on each software system. In order to get to a "short-list" of candidate systems, the team engaged in a market analysis process which was used to screen the system by technical, functional and vendor specific criteria. The arrival of information and the evaluation of each offering were performed as a concurrent process across the team. As the RFI responses were received a more detailed evaluation process was undertaken to review the cost, technical function and vendor capacity to serve as the finalist. 


\begin{tabular}{|c|c|}
\hline Company & Web Site Address \\
\hline ABBASOFT Technologies Inc. & http://abbasoft.com \\
\hline Aria Systems, Inc. & http://www.ariany.com/index \\
\hline Bowen \& Groves Inc. & http://www.bowen-groves.com \\
\hline Consona Corporation & http://www.consona.com/Consona \\
\hline DBA Software Inc. & http://www.jaaltd.com \\
\hline Epicor Software Corporation & http://www.epicor.com/Products/Pages/Vista \\
\hline Exact Software & http://www.exactamerica.com/alliance \\
\hline Exact Software & http://www.exactamerica.com/jobboss \\
\hline Freedom Applications LTD & http://www.freedomapps.com \\
\hline Henning Industrial Software Inc. & http://www.henningsoftware.com \\
\hline In-Style Software Inc. & http://www.instylesoft.com \\
\hline Infor ERP Solutions & http://www.infor.com/ \\
\hline ManEx Inc. & http://www.manex.com \\
\hline Manufacturing Advisors, LLC & http://www.mfgcatalyst.com \\
\hline Microsoft Dynamics & http://www.microsoft.com \\
\hline MISys Inc. & http://www.misysinc.com \\
\hline OmegaCube Technologies & http://www.omegacube.com \\
\hline Pentagon 2000SQL & http://www.pentagon2000.com/ \\
\hline Positive Business Solutions Inc. & http://www.quickbooksenterprise.intuit.com \\
\hline QuickBooks Enterprise & http://www.pbsinet.com/pbs/index \\
\hline Sage software & http://www.sageproerp.com \\
\hline Seradex & http://www.seradex.com \\
\hline Shoptech Software Corp. & http://www.shoptech.com \\
\hline $\begin{array}{l}\text { Vision ERP Group North America } \\
\text { Weber Systems Inc. }\end{array}$ & $\begin{array}{l}\text { http://www.tgiltd.com/erp_comparison_list } \\
\text { http://www.webersystems.com }\end{array}$ \\
\hline
\end{tabular}

Figure 41 - First Stage Sort Long-List

In this case study the long list included twenty-six candidate systems, which were asked to respond to an RFI process mentioned earlier, and a preliminary vendor qualification process was begun, using publicly available and published business information, in order to establish corporate financial status. Second stage sorting consisted of evaluation of these data and the RFI responses to develop a short-list of viable products. These 
vendors were asked to participate in both structured and ad-hoc information gathering processes, including organized demonstrations, principally online.

The third stage, development of an ultimate candidate group, included early stage negotiations with each finalist vendor, to establish best pricing and to determine the timelines and actual proposal for resource use in the implementation phase. Each candidate system vendor that reached this stage was asked to provide recent references, for system implementations at similar enterprises to $\mathrm{ABC}$, and any publicly available financial status information, such as annual and quarterly reports. The team obtained Dun and Bradstreet ${ }^{\circledR}$ financial reports in addition to the vendor supplied information, and vetted the references independently.

Step 10: In order to provide a clear picture of the requirements, they were sorted into four categories, to facilitate evaluation. A Delphi committee was assembled consisting of the key consultants and for $\mathrm{ABC}$; the chief financial officer, chief operations officer, director of manufacturing, director of quality. This group was briefed and tasked with an evaluation and comparison process to rank each of the requirements by order of importance, and to extract any requirements that did not have sufficient significance to $\mathrm{ABC}$ 's needs. In addition, the team was asked to assemble a requirements set, again ranked in order of importance, of the reports that would be required to be produced by the ERP system and to indicate the frequency of occurrence of those reports. An example worksheet for the accounting system requirements ranking process is shown below, and the full set of ranked requirements are included in Appendix E. 


\begin{tabular}{|c|c|c|c|c|}
\hline & \multicolumn{2}{|l|}{ SUMMARY OF ABC ERP REQUIREMENTS } & & \multirow[b]{2}{*}{$\begin{array}{l}\text { Not very } \\
\text { important }\end{array}$} \\
\hline & & $\begin{array}{l}\text { Extremely } \\
\text { Important }\end{array}$ & & \\
\hline & Rate Requirements as follows & 10 & $\ldots \ldots \ldots$ & 1 \\
\hline & & & & \\
\hline & Accounting Requirements & & & \\
\hline 1 & ERP System needs to communicate with ADP. & & 10 & \\
\hline 2 & 16-17 character long for contract number is required & & 10 & \\
\hline 3 & Reduced Accounts Payable workload & & 10 & \\
\hline 4 & Reduced General ledger workload & & 10 & \\
\hline 5 & Custom financial reporting & & 10 & \\
\hline 6 & Sales commission to be considered & & 10 & \\
\hline 7 & Possibility of off-site operation. (Two different companies) & & 10 & \\
\hline 8 & Departmental profit and loss statements. & & 10 & \\
\hline 9 & Determine project profitability. & & 10 & \\
\hline 10 & Generate vouchers and invoices. & & 10 & \\
\hline 11 & Print monthly checks. & & 10 & \\
\hline 12 & Track commissions, invoices. & & 10 & \\
\hline 13 & Earned Value Management System (EVMS) & & 10 & \\
\hline 14 & Value Stream Mapping capabilities (VSM) & & 10 & \\
\hline 15 & Government Cost Account (GCA)- Timekeeping (time by project) & & 10 & \\
\hline 16 & GCA - Project Costing (tracking of all materials and labor by project) & & 10 & \\
\hline 17 & GCA - Multiple Overhead Pools \& one G\&A & & 10 & \\
\hline 18 & GCA - Time and Material (Revenue and Billing) & & 10 & \\
\hline 19 & $\begin{array}{l}\text { GCA - Cost - must calculate revenue on labor \& materials using actual } \\
\text { direct costs plus Billing Overhead + Billing G\&A rate by year }\end{array}$ & & 10 & \\
\hline 20 & GCA - FFP - system must accommodate milestone billing on projects & & 10 & \\
\hline 21 & Reduced Accounts Receivable workload & & 9 & \\
\hline 22 & Accounting dashboard & & 8.5 & \\
\hline 23 & $\begin{array}{l}\text { Interact with other systems and applications (i.e. inventory, work order } \\
\text { tracking, work progress, and shipping). }\end{array}$ & & 8 & \\
\hline 24 & Determine ROI. & & 7 & \\
\hline 25 & Financial report and cost tracking & & 6 & \\
\hline 26 & Ability to store and use recurring invoices. & & 5 & \\
\hline 27 & Consistency of data across reports & & 3 & \\
\hline 28 & Employee time sheets. & & 3 & \\
\hline 29 & $\begin{array}{l}\text { Generate book to bill ratios, P \& L report, and income and expenses } \\
\text { report (biweekly), general \& administration cost (quarterly). }\end{array}$ & & 2 & \\
\hline
\end{tabular}

Figure 42 - Sample Requirements Ranking Worksheet.

Step 11: The survey was undertaken to identify and bench-mark the selection process to similar business enterprises that had successfully selected and implemented ERP systems in the recent past. 
Numerous studies have described categorical and specific causal factors for ERP system implementation failure, however there is a paucity of studies for small business enterprises.. In order to have a high degree of compatibility of the survey results to the targeted business sector, the survey was directed to a group of small manufacturing businesses, with successful recent experiences in the selection and implementation of ERP systems. Other qualifying factors were:

- Annual Sales Volume (less than $\$ 100 \mathrm{~m}$ )

- Types of Products (components, sub-assemblies, design-build products etc)

- Number of employees (less than 500)

- Years in business (less than 30)

Twenty three companies were invited to participate in the survey, fourteen companies agreed to provide answers. Of the survey responses received one was found to be inconsistently completed, providing a response rate of (56.5\%) which is acceptable and exceeds the rate of response of other studies in this area (Paulraj, 2005) 23.2\%; (Krause, D.R., Pagell,M., \& Curkovic,S., 2001) 19.6\%.

All of the responding companies have been in business for more than five years, and $69 \%$ are grossing revenues of more than $\$ 10 \mathrm{~m}$. The profiles also show that all of the companies have employee levels well below the Small Business Administration's (SBA) graduation level of 500 , and all have revenues below $\$ 100 \mathrm{~m}$, making all the companies qualified 'small' businesses by the SBA definition. 
Table 1 - Business Profile of Responding Companies.

\section{Annual Sales Volume:}

\begin{tabular}{|l|l|l|}
\hline Less than $\$ 10 \mathrm{~m}$ & 4 & $30.77 \%$ \\
\hline$\$ 10 \mathrm{~m}-\$ 50 \mathrm{~m}$ & 6 & $46.15 \%$ \\
\hline$\$ 50 \mathrm{~m}-\$ 100 \mathrm{~m}$ & 3 & $23.08 \%$ \\
\hline Greater than $\$ 100 \mathrm{~m}$ & 0 & $0.00 \%$ \\
\hline
\end{tabular}

\section{Employees:}

\begin{tabular}{|l|l|l|}
\hline Less than 100 & 6 & $46.15 \%$ \\
\hline $101-200$ & 7 & $53.85 \%$ \\
\hline $201-500$ & 0 & $0.00 \%$ \\
\hline
\end{tabular}

\section{Product: (Check all that apply)}

\begin{tabular}{|l|l|l|}
\hline Components & 9 & $69.23 \%$ \\
\hline Sub-Assemblies & 7 & $53.85 \%$ \\
\hline Major Assemblies & 4 & $30.77 \%$ \\
\hline Other & 8 & $61.54 \%$ \\
\hline
\end{tabular}

\section{Years in Business:}

\begin{tabular}{|l|l|l|}
\hline $0-5$ years & & $0.00 \%$ \\
\hline $5+$ to 10 years & 4 & $30.77 \%$ \\
\hline $10+$ to 15 years & 8 & $61.54 \%$ \\
\hline $15+$ to 20 years & 1 & $7.69 \%$ \\
\hline $20+$ to 30 years & & $0.00 \%$ \\
\hline
\end{tabular}

Since, the data were derived from a targeted group, the survey produced results that are tightly correlated and show good internal consistency. In the data reporting, Cronbach's alpha is reported for the data set as whole, acceptable values of alpha are taken to be those greater than 0.6 which is supported in the literature as the lowest acceptable value in practical terms for this coefficient, although the coefficient can take on values of ; $-\infty \leq \boldsymbol{\alpha} \leq \mathbf{1}$ (Cronbach, 1951) (Gliem, 2003). Cronbach's coefficient measures the 
internal consistency of the data, the closer it is to 1.0 the greater the consistency. The reported values of Cronbach's alpha for the data relating to the nine constructs under evaluation range from 0.6101 to 0.8609 ; all are within the acceptable range.

Some of the largest standard deviations, showing a diversity of opinion with respect to the issues in question were found in the change strategy section. The spread of opinions regarding strategic concepts is not altogether surprising, given that small companies tend to have residual attitudes about how to do things best. Two issues seem to have the widest diversity of opinion. Firstly, acceptance that strategic planning as it relates to the IT system is a continuous process scores low overall, tending to indicate a reluctance to elevate the IT component of the business as a part of the overall ERP philosophy. Secondly, while there is a strong understanding that, getting the most out of an ERP system is founded in a strategic approach (demonstrated by the high mean score and low standard deviation in question 7), the scoring of question 2 suggests there is a spread of opinion as to whether to begin that planning at the divisional level. Since, these companies are already benefitting from their ERP systems it is not surprising that the process of utilizing and planning for those benefits, reflected in questions; 1,4 and 7, is well supported by these findings. 
Table 2 - Change Strategy

\begin{tabular}{|c|c|c|c|}
\hline Element & Question & Mean & $\begin{array}{l}\text { Standard } \\
\text { Deviation }\end{array}$ \\
\hline 1 & Organizational IT planning has a written procedure. & 5.77 & 0.697 \\
\hline 2 & The strategic plans of each division include the ERP. & 6.00 & 1.038 \\
\hline 3 & The overall IT plan includes the concerns of each division. & 6.15 & 0.769 \\
\hline 4 & $\begin{array}{l}\text { Managers consider the potential for ERP efficiency in } \\
\text { strategic plans. }\end{array}$ & 5.69 & 0.606 \\
\hline 5 & The ERP selection was based on strategic plan objectives & 6.15 & 0.533 \\
\hline 6 & Strategic planning for IT is a continuous process. & 4.69 & 1.323 \\
\hline \multirow[t]{2}{*}{7} & IT capabilities are reviewed against strategic objectives. & 5.69 & 0.462 \\
\hline & & $\begin{array}{l}\text { Cronbac } \\
\text { Alpha }=\end{array}$ & 0.6174 \\
\hline
\end{tabular}

As a class, companies in the subject business sector tend to have more sophisticated systems and greater technical ability than equivalent small businesses in other sectors, which are reflected in the overall acceptance within these small businesses of the importance of the strategic approach to ERP system use.

Table 3 shows the results regarding the importance of executive buy-in and the necessity of proactive support to make the ERP project successful. An under resourced project will almost inevitably fail, and since the resources are ultimately allocated by authority of the senior management team, the corporate perception of "what the senior managers do versus what they say" must have consistency. 
Table 3 - Executive Facilitation

\begin{tabular}{|c|c|c|c|}
\hline Element & Question & Mean & $\begin{array}{l}\text { Standard } \\
\text { Deviation }\end{array}$ \\
\hline 1 & $\begin{array}{l}\text { Executives require ERP to have priority over operational } \\
\text { issues. }\end{array}$ & 5.23 & 0.697 \\
\hline 2 & Executives are strong advocates for the value of ERP. & 4.92 & 0.828 \\
\hline 3 & $\begin{array}{l}\text { Senior managers have clearly expressed the ERP's business } \\
\text { objectives. }\end{array}$ & 5.00 & 0.555 \\
\hline 4 & Executives are always promoting the ERP process. & 4.92 & 0.828 \\
\hline 5 & $\begin{array}{l}\text { The resource needs of the ERP support process is recognized } \\
\text { by senior management. }\end{array}$ & 4.69 & 0.606 \\
\hline 6 & $\begin{array}{l}\text { ERP goals are recognized and supported by all levels of } \\
\text { management. }\end{array}$ & 4.85 & 0.533 \\
\hline 7 & Executives are well informed on the potential ERP benefits. & 5.00 & 0.784 \\
\hline & & $\begin{array}{l}\text { Cronbach's } \\
\text { Alpha }=\end{array}$ & 0.6981 \\
\hline
\end{tabular}

In the survey the corporate perception of the concurrence of these themes, is well supported, mean scores ranging from 4.69 to 5.23 indicating strong agreement that the senior management teams involved in these companies are demonstrating the right kind of consistency in their message and in their actions to support the ERP project.

These results are in agreement with broader surveys, which have tended to survey larger, better resourced businesses (Gupta, 2000) (Correa, 2007) (Chang \& et al., 2008). The relatively high score in Question 1 show that, for this set of executives at least, there is a clear message about the organizational priority of the ERP system. 
Table 4 - Project Management Competency

\begin{tabular}{|c|c|c|c|}
\hline Element & Question & Mean & $\begin{array}{l}\text { Standard } \\
\text { Deviation }\end{array}$ \\
\hline 1 & $\begin{array}{l}\text { Project team member's individual responsibilities are well } \\
\text { defined. }\end{array}$ & 6.00 & 0.555 \\
\hline 2 & The status of project tasks are measured and communicated. & 5.23 & 0.576 \\
\hline 3 & $\begin{array}{l}\text { The ERP project leader can track the status of tasks at all } \\
\text { stages. }\end{array}$ & 5.62 & 0.738 \\
\hline 4 & $\begin{array}{l}\text { The task objectives of all elements of the ERP project are } \\
\text { well defined. }\end{array}$ & 5.69 & 0.821 \\
\hline 5 & There is a process to track performance of contractors. & 5.23 & 0.697 \\
\hline 6 & $\begin{array}{l}\text { The project manager for the ERP process is well versed in } \\
\text { project management. }\end{array}$ & 6.00 & 0.679 \\
\hline 7 & $\begin{array}{l}\text { The ERP project manager has instituted strong change } \\
\text { control to prevent scope creep. }\end{array}$ & 5.77 & 0.697 \\
\hline & & $\begin{array}{l}\text { Cronbach's } \\
\text { Alpha }=\end{array}$ & 0.8609 \\
\hline
\end{tabular}

The implementation of an ERP is not a conventional project; its end point is not well defined. One could assert that the go-live is the end of the implementation, but practical experience and many studies have shown that the cultural changes wrought by an ERP introduction are continuous and permanent (Correa, 2007) (Gupta, 2000).

Small manufacturing businesses, especially those that have a high product mix, are necessarily well versed in the principles of project management. Balancing, resource availability, quality of the outcomes, and the timeliness of the production process are key 
competitive differentiators for success. The presence of strong capabilities in project management is a welcome finding in this survey. It is a hallmark of successful small businesses that the importance of individuals continues to be recognized, small teams in particular rely on their star players to produce the greatest value. Clearly the corporations in review have a strong commitment to this skill area.

It has been suggested that larger companies are more likely to have good project management skills, since they have larger resource bases to draw on; this viewing of this class of small business shows that as a key survival strategy, project management skills are highly refined and equally present within the small business community. The data show that the sample group is broadly satisfied with the IT skills, and the service level provided by their in-house staff, and will accommodate fluctuations in resource demand by hiring outside consultants, although the lower mean score for this element suggests a reluctance or lower level of trust. The lower level of enthusiasm for consultants can be attributed either a lack of perceived competence or to a natural reluctance to spend the company's funds on a temporary workforce. 
Table 5 - IT Based Competency

\begin{tabular}{|c|c|c|c|}
\hline Element & Question & Mean & $\begin{array}{l}\text { Standard } \\
\text { Deviation }\end{array}$ \\
\hline 1 & ERP upgrades are efficiently incorporated by IT staff. & 5.00 & 0.679 \\
\hline 2 & $\begin{array}{l}\text { IT staff are systematically upgrading their skills through } \\
\text { training. }\end{array}$ & 5.31 & 0.606 \\
\hline 3 & The IT staff provides good value to the organization. & 5.15 & 0.662 \\
\hline 4 & The IT staff is an expert resource to the business. & 5.15 & 0.533 \\
\hline 5 & $\begin{array}{l}\text { The IT team is well versed in the ERP software and } \\
\text { customizations. }\end{array}$ & 5.15 & 0.662 \\
\hline 6 & $\begin{array}{l}\text { The IT staff can evaluate proposed ERP related technical } \\
\text { changes and respond appropriately. }\end{array}$ & 5.54 & 0.499 \\
\hline 7 & $\begin{array}{l}\text { Consultants are contracted to support the IT staff for high } \\
\text { demand periods. }\end{array}$ & 4.92 & 0.615 \\
\hline & & $\begin{array}{l}\text { Cronbach's } \\
\text { Alpha }=\end{array}$ & 0.6847 \\
\hline
\end{tabular}

Small businesses, and especially those that are successful, must make the most of the employees. Small increases in productivity can make big differences in the company's bottom line. These data show that there is a strong recognition of the required skills, particularly of the IT staff, with respect to the ERP project. More surprisingly, there is a low response to the disciplinary obligations dealing with low staff competence levels. There is an even greater reluctance to disciplinary intervention for managers. The responses are backed up by the response to the suggestion that ERP project staff should see greater rewards for their involvement in the project. These attitudes can be explained 
by the fact that, small businesses invest heavily in personnel, and each person is seen as an individual. Consequently, the imperative to exercise discipline is mitigated by several factors; length of service, prior performance, ability to do multiple jobs, character and personality related attributes.

Table 6 - Staffing

\begin{tabular}{lll} 
Element Question & $\begin{array}{l}\text { Standard } \\
\text { Deviation }\end{array}$ \\
\hline
\end{tabular}

$1 \quad$ ERP team members have to understand that the project is their highest priority.

$5.15 \quad 0.662$

Senior managers with little ERP knowledge should limit their

4.54

0.499 involvement.

3 Employees who cannot accommodate the ERP related

4.46

0.634 changes should be reassigned or terminated.

4 Managers who cannot accommodate the ERP related changes

4.38

0.625 should be reassigned or terminated.

5 When workloads are high, or there is inadequate internal knowledge of an ERP issue, consultants are hired.

4.85

ERP team members who excel in terms of performance should be incentivized.

7 Inadequate IT competence can negatively affect the ERP

5.62

0.487 implementation process.

8 ERP implementation requires the IT staff to have a high

5.62

0.625 degree of adaptability and the ability to learn. 
These factors are even more present in managers who have frequently been hired from within and are examples of the work attributes that the senior management wants to promote in the staff.

Table 7 - Training

Element Question

\begin{tabular}{ll} 
Mean & $\begin{array}{l}\text { Standard } \\
\text { Deviation }\end{array}$ \\
\hline
\end{tabular}

$1 \quad$ ERP users have been provided with formal training.

5.85

0.533

2

All ERP system users have received, at least, basic training in

6.31

0.462 the ERP systems.

3 Training covers all elements of the business system, not just

5.38 software familiarity with the system.

$4 \quad$ HR and performance records are maintained to determine that employees have received the appropriate training for their positions.

5

The training program is ongoing, with access to refresher and new training offerings.

5.54

0.499

Consultants are used for training that is beyond the ability or

5.62

0.487 knowledge of internal resources.

7 The training needs of all classes of user were identified early in the ERP implementation process. applicability to each assignment. 
Disciplinary actions or rewards that single out individuals can be counterproductive to good team development and organizational harmony, particularly if those rewards or the discipline is associated with a single axis of the person's overall abilities.ERP training issues are frequently identified as being among the root causes of implementation failures (Motwani J., 2005) (Themistocleous, Irani, O’Keefe, \& Paul, 2001) (Kumar V. M., 2003). The aggregate level of importance and close agreement of the best practices to adopt to mitigate failure is demonstrated by the results of the training survey component.

A very high degree of agreement, shown by narrow standard deviations, and high mean values, suggests a common view of the importance of training. All sample group member's assigned great significance to the concept of universal basic training. Almost as high agreement is seen in the necessity of that training being underpinned with formal sessions for the ERP user community. The survey suggests that there is agreement that customized training should be adopted wherever possible. There was less certainty that the overall training needs were well identified during the implementation process, suggesting that this group of ERP users may have encountered unanticipated training costs. Training is one area where all members of the survey group acknowledged the value of external consultants, and that training is and needs to be an ongoing process for all staff members. In addition, there is good recognition that the training must cover all aspects of the business process that are affected by the ERP, which implies that there should some degree of cross training involved to allow a broad understanding of ERP throughout the organization. 
The attributes related to BPR process knowledge, may be one of the critical factors which have made these small businesses competitive and successful in their implementation projects. The commitment to process and documentation of the processes is in high agreement with the expected performance levels of flourishing companies. The team related questions generated high levels of agreement with the constructs, and point to the outstanding team work that is essential to achieving goals, and to the cross-disciplinary transfer of knowledge that is so vital to make small businesses successful.

Table 8 - BPR Competency

Element Question
Standard

Mean Deviation

1 There is a high level of business process knowledge in the ERP team.

5.69

0.606

Managers do a good job in analyzing business process effects

5.00

0.679 in producing customer benefits.

3 Business processes are redesigned before the ERP system is implemented.

$5.69 \quad 0.606$

$4 \quad$ All BPR teams are drawn from cross functional areas.

$5.15 \quad 0.533$

5

ERP team work processes are formally documented.

$5.77 \quad 0.576$

Efficiency improvements are addressed by BPR.

$5.38 \quad 0.487$

7

The operational effect of an individual's performance on other

5.46

0.499 processes is well understood by employees. 
One issue shown by the statistics is the relatively widespread conviction that managers are doing a good job in translating BPR into a value proposition for the clients of the companies; it is instructive that the mean score was not higher, since the ultimate purpose of any process change must create value inside and outside the organization. Apart from a disconnect in the understanding of the value of module selection in the ERP selection process there is a high level of agreement that software selection is crucial feature of a successful ERP project overall.

This disagreement may be due to the fact that most small businesses elect to purchase systems that are less recognizably 'modular' than the large system like SAP. The data show a high level of confirmation that the software development, testing, troubleshooting and data migration have to be well planned processes.

It is frequently recorded in the literature that these issues are a cause of concern, and are commonly contributory factors to delayed or failed implementations (Al-Mashari M. , Implementing ERP through SAP $\mathrm{R} / 3$ : A process change management (PCM) perspective., 2002) (Chang \& et al., 2008) (Hong \& Kim, 2002). These data also show that for the purposes of the ERP implementation process, consultants are regarded as an appropriate and preferred method to bring in the knowledge and skills to help the company with its selection process. In addition, the working relationship of the company and its chosen vendor are recognized as a key strength that can be established to help ensure successful identification of the appropriate software system. 
Table 9 - Software Selection

\begin{tabular}{|c|c|c|c|}
\hline Element & Question & Mean & $\begin{array}{l}\text { Standard } \\
\text { Deviation }\end{array}$ \\
\hline 1 & $\begin{array}{l}\text { The complete ERP architecture should be designed before go } \\
\text { live. }\end{array}$ & 5.23 & 0.421 \\
\hline 2 & $\begin{array}{l}\text { Data migration and housekeeping should be a planned } \\
\text { process. }\end{array}$ & 5.69 & 0.606 \\
\hline 3 & $\begin{array}{l}\text { The selection process should determine which modules are to } \\
\text { be acquired. }\end{array}$ & 4.77 & 0.421 \\
\hline 4 & $\begin{array}{l}\text { Information flow between system modules should be } \\
\text { seamless. }\end{array}$ & 5.62 & 0.487 \\
\hline 5 & $\begin{array}{l}\text { The ERP system should eliminate redundant data entry } \\
\text { processes. }\end{array}$ & 5.38 & 0.487 \\
\hline 6 & $\begin{array}{l}\text { Consultants are required to supplement gaps in in-house } \\
\text { knowledge. }\end{array}$ & 5.54 & 0.499 \\
\hline 7 & $\begin{array}{l}\text { Strong relationships with vendors and consultants should be } \\
\text { established to aid in the resolution of problems. }\end{array}$ & 5.15 & 0.361 \\
\hline 8 & $\begin{array}{l}\text { Software testing and rigorous process simulation aids in } \\
\text { preparing for the go-live action. }\end{array}$ & 5.46 & 0.499 \\
\hline 9 & $\begin{array}{l}\text { ERP system software development, testing and } \\
\text { troubleshooting are crucial to the preparation process. }\end{array}$ & 5.62 & 0.487 \\
\hline & & $\begin{array}{l}\text { Cronbach's } \\
\text { Alpha }=\end{array}$ & 0.7022 \\
\hline
\end{tabular}


Table 10 - Project Communication and Support

\begin{tabular}{|c|c|c|c|}
\hline Element & Question & Mean & $\begin{array}{l}\text { Standard } \\
\text { Deviation }\end{array}$ \\
\hline 1 & $\begin{array}{l}\text { Employee concerns about the ERP process are actively } \\
\text { addressed by managers }\end{array}$ & 5.77 & 0.697 \\
\hline 2 & $\begin{array}{l}\text { ERP roles and responsibilities are communicated to each } \\
\text { employee. }\end{array}$ & 6.15 & 0.533 \\
\hline 3 & $\begin{array}{l}\text { The ERP team is accessible for employee concerns about job } \\
\text { changes. }\end{array}$ & 6.15 & 0.769 \\
\hline 4 & $\begin{array}{l}\text { Employees understand how their jobs fit into the new ERP } \\
\text { structure. }\end{array}$ & 5.69 & 0.606 \\
\hline 5 & $\begin{array}{l}\text { Excellent communication is crucial to effective ERP } \\
\text { implementation. }\end{array}$ & 6.23 & 0.576 \\
\hline 6 & $\begin{array}{l}\text { Structural and cultural changes to the business during ERP } \\
\text { implantation should be a managed process. }\end{array}$ & 5.85 & 0.662 \\
\hline 7 & $\begin{array}{l}\text { Requirements, approvals, commentary and responses should } \\
\text { be elicited as inputs from the ERP user community. }\end{array}$ & 5.69 & 0.462 \\
\hline 8 & $\begin{array}{l}\text { A companywide culture, promoting common goals and } \\
\text { objectives is a strong driver for success. }\end{array}$ & 6.00 & 0.784 \\
\hline & & $\begin{array}{l}\text { Cronbach's } \\
\text { Alpha }=\end{array}$ & 0.6821 \\
\hline
\end{tabular}

The importance of communication, internally, across divisional boundaries, and with all external participants is emphasized by these results. The importance of teamwork and establishing trust within the organization, through; engaged managers and well informed 
employees is held to highly important by these survey respondents. The clear understanding of the roles and responsibilities of each employee is seen as one of the key factors in successful implementation, the related question has a mean of 6.15 and a very narrow standard deviation suggesting this is a strongly held and widespread belief.

Issues related to corporate culture, access to expert guidance, and effective and open responses from managers regarding employee concerns were also shown to be important in the evaluations. This finding comports with the characteristics of successful small businesses; employees are valued as individuals and as sources of value to the organization, and expect to be effective participants in change.

Step 12: Each of the short-listed vendors was asked to participate in a structured demonstration in which they were asked to develop the full life cycle of a set of typical business processes and their related transactions. This demonstration provided vital information to clarify whether ABC's needs were being specifically addressed by each system, and gave the evaluation teams the ability to compare, qualitatively, how closely each vendor's system conformed to $\mathrm{ABC}$ 's expectations. The scenarios and demonstration script were developed by the selection team and included the expectation that all the requirements that were identified in step 10 should be demonstrated. The performance tests were designed to test whether the candidate ERP systems could successfully demonstrate that the requirements of the company had been met, and to what degree they had matched the requirements. 
Step 13: Standardized scoring instruments, spreadsheets, were developed to allow the evaluators to provide their estimation of the candidate ERP systems against the individual requirements. In this case, there were four categories of requirements; accounting, manufacturing, other requirements and reports, containing; twenty nine accounting system requirements, forty five manufacturing requirements, twenty other requirements and twenty three report requirements.

Step 14: A key consideration to the overall effectiveness of the selection process was cost conscious utilization of team resources. Selection of the right staff team members at $\mathrm{ABC}$ required careful coordination with departmental managers, so that the best prepared individuals were identified for the evaluation process. This assignment was negotiated with the individual managers to ensure the evaluators continued availability over the life of the evaluation process in order to maintain continuity. Especially in a small firm, the availability of resources is a critical issue. However, the return on investment to the company of this level of participation is twofold; firstly, it contributes to the corporate memory and staff knowledge of the decision making process and why certain options were selected and others rejected is in place, and secondly, participation is a key factor in reducing negative perceptions regarding the new software system, and creating a climate of acceptance for the new system. For this case study eleven finalists were short-listed, and invited to participate in the demonstration and evaluation phase.

As a practical matter this system of analysis is workable only for a modest number of candidate systems, since the number of requirements can grow to be unwieldy if the 
number of ranking categories is expanded. For the problem in question, the final tally of requirements was 117. There were four category ranking decisions; in addition 117 requirement ranking decisions per expert were required, with typically five experts per ranking review. The subsequent evaluation of the performance requirements, typically utilizing 4 staff members per candidate system with 11 candidate systems under review, required 5148 individual decisions. For this fairly modest short list of 11 systems in review, a total of 5737 individual weighting and evaluation judgments were required.

In addition, it should be noted that the process of gathering the selection data is highly labor intensive, and the veracity of the data depends on timely acquisition of highly volatile financial information, and the concurrence of the vendors to participate in the evaluation process demonstration testing envisaged in this methodology. The ERP system vendor demonstrations were scheduled over a three week timeframe, and all were successfully evaluated.

Step 15: The selection team assembled all the evaluator's responses, for each ERP system, and determined an aggregate score for each requirement. These scores were modified in accordance with the weighting developed earlier. The scores were compared to the hypothetical perfect score, and each ERP system's percentage of functionality, with respect to the requirements, was calculated. 


\begin{tabular}{|l|r|r|r|r|r|}
\hline & \multicolumn{1}{|c|}{ System A } & \multicolumn{1}{c|}{ System B System C } & \multicolumn{1}{l|}{ System D System E } \\
\hline \multirow{3}{*}{ Weighted Utility Score } & & & & & \\
\cline { 2 - 6 } & 82.62 & 83.95 & 82.51 & 73.18 & 76.4 \\
\cline { 2 - 6 } & & & & & \\
\cline { 2 - 6 } Utilty Percentage & $75.42 \%$ & $76.64 \%$ & $75.32 \%$ & $66.81 \%$ & $69.75 \%$ \\
\hline
\end{tabular}

Figure 43 - Finalist ERP Systems Weighted Evaluation Scores

Step 16: Based on the management review of the shortlisted ERP system performance results, the top five systems were regarded as having sufficient capability, in the requirement responses, to be selected as final candidate systems. The threshold for performance, against the requirements, was set at $66 \%$.

Step 17/18/19/20: An RFP, requesting best and final pricing, was issued to the five finalist systems. The purpose of the RFP was to identify the full cost of ownership, attributable to the ERP vendor. Using the information gathered in earlier stages of the selection process, the internal costs for five years of operation were assessed. For the purposes of the evaluation a weighted utility score is regarded as equivalent if it is within a range of two percent. System alternatives A, B. and C are all functionally equivalent. Step 21: Since, alternatives A, B and C are equivalent in terms of functional fit, and we are directed to adopt a logic which purchases the maximum functionality, with a secondary condition of cost, we must determine which of these equivalent systems has the least cost over a five year timeframe. 
Step 21: As demonstrated in the figure below, the least cost option system is system C, which was the selected system.

\begin{tabular}{|c|c|c|c|c|c|}
\hline & System A & System B & System C & System D & System E \\
\hline Software Cost & $\$ 100,000.00$ & $\$ 90,767.00$ & $\$ 82,150.00$ & $\$ 31,475.00$ & $\$ 32,500.00$ \\
\hline Implementation & $\$ 160,000.00$ & $\$ 46,210.00$ & $\$ 48,530.00$ & $\$ 12,500.00$ & $\$ 12,000.00$ \\
\hline 1st Year Maintenance & $\$ 18,000.00$ & $\$ 18,153.00$ & Included & Included & $\$ 6,300.00$ \\
\hline Discounts & & & $-\$ 44,680.00$ & & $-\$ 1,000.00$ \\
\hline Total Initial Investment & $\$ 260,000.00$ & $\$ 136,977.00$ & $\$ 130,680.00$ & $\$ 43,975.00$ & $\$ 44,500.00$ \\
\hline Annual Maintenance Cost & $\$ 18,000.00$ & $\$ 18,153.00$ & $\$ 15,870.00$ & $\$ 0.00$ & $\$ 6,300.00$ \\
\hline Internal Costs of Ownership per Year & $\$ 26,000.00$ & $\$ 24,220.00$ & $\$ 18,780.00$ & $\$ 15,600.00$ & $\$ 15,600.00$ \\
\hline 5 Year Ownership Cost & $\$ 480,000.00$ & $\$ 348,842.00$ & $\$ 303,930.00$ & $\$ 121,975.00$ & $\$ 154,000.00$ \\
\hline Utility Score & 82.62 & 83.95 & 82.51 & 73.18 & 76.4 \\
\hline \multirow[t]{5}{*}{ Utility Percentage } & $75.42 \%$ & $76.64 \%$ & $75.32 \%$ & $66.81 \%$ & $69.75 \%$ \\
\hline & & & & & \\
\hline & & & & & \\
\hline & System A & System B & System C & System D & System E \\
\hline & NPV Cost & NPV Cost & NPV Cost & NPV Cost & NPV Cost \\
\hline Software Cost & $\$ 100,000.00$ & $\$ 90,767.00$ & $\$ 82,150.00$ & $\$ 31,475.00$ & $\$ 32,500.00$ \\
\hline Implementation ( 2 years) & $\$ 150,887.57$ & $\$ 43,578.22$ & $\$ 45,766.09$ & $\$ 11,788.09$ & $\$ 11,316.57$ \\
\hline 1st Year Maintenance + Internal Costs & $\$ 44,000.00$ & $\$ 42,373.00$ & $\$ 34,650.00$ & $\$ 15,600.00$ & $\$ 21,900.00$ \\
\hline 2nd Year Maintenance + Internal Costs & $\$ 42,307.69$ & $\$ 40,743.27$ & $\$ 33,317.31$ & $\$ 15,000.00$ & $\$ 21,057.69$ \\
\hline 3rd Year Maintenance + Internal Costs & $\$ 40,680.47$ & $\$ 39,176.22$ & $\$ 32,035.87$ & $\$ 14,423.08$ & $\$ 20,247.78$ \\
\hline 4th Year Maintenance + Internal Costs & $\$ 39,115.84$ & $\$ 37,669.44$ & $\$ 30,803.72$ & $\$ 13,868.34$ & $\$ 19,469.02$ \\
\hline 5th Year Maintenance + Internal Costs & $\$ 37,611.38$ & $\$ 36,220.62$ & $\$ 29,618.97$ & $\$ 13,334.95$ & $\$ 18,720.21$ \\
\hline Discounts & & & $-\$ 44,680.00$ & & $-\$ 1,000.00$ \\
\hline 5 Year Ownership Cost & $\$ 454,602.96$ & $\$ 330,527.77$ & $\$ 243,661.96$ & $\$ 115,489.46$ & $\$ 144,211.27$ \\
\hline Utility Score & 82.62 & 83.95 & 82.51 & 73.18 & 76.4 \\
\hline Utility Percentage & $75.42 \%$ & $76.64 \%$ & $75.32 \%$ & $66.81 \%$ & $69.75 \%$ \\
\hline
\end{tabular}

Figure 44 - ERP System Selection Spreadsheet

Step 22: The underlying assumption for the effective use of the methodology is that the requirements gathering process is sufficiently rigorous to obtain all the required needs of the company, and that they represent the central basis to which the ERP system under review can be compared. Unfortunately, the requirements can grow to become very large data sets, and in order to test, and improve the methodology, as required by Thomann's 
approach, we tried to establish whether a smaller group of combined requirements could be substituted in future applications, and how they might be combined into a shorter list for evaluation. The most important facet of working with small business enterprises is the necessity to understand that resource demands are always high, and that any competing demand on those resources is a burden to the operational efficiency of the company. Since a large proportion of the time and effort of the ABC staff was dedicated to requirements gathering, evaluation and demonstration activities, an attempt was made to explore the significance of the requirements through experimental factor analysis.

The techniques of factor analysis are well documented and allow technical evaluation for multivariate data responses; they were originally developed to facilitate research performed in the area of psychometric analysis. In this study we have evaluated each category of the requirements for the case study example through the techniques of principal component analysis (PCA). The objective is to attempt to identify the distribution of variability within the data sets, and to determine whether a viable set of combined factors can be developed for the requirement variables which would reduce the overall burden in evaluating the system utility score.

The primary intent of principal component analysis is to determine whether the correlation between variables is sufficient to allow their combination. If the contribution of any given variable is negligible, that variable may be excluded or abandoned in future studies. In addition, if sufficient information can be retained by reducing the combined factors to two or three, then the visualization of the contribution of the factors can be 
achieved through graphical methods. The fully evaluated performance score matrix for the category "Other Requirements" is included as Figure 15 to demonstrate the process of the PCA.

\begin{tabular}{|c|c|c|c|c|c|c|c|c|c|c|c|}
\hline Other Requirements & A & B & C & D & $\mathrm{E}$ & $\mathrm{F}$ & G & $\mathrm{H}$ & 1 & J & $\mathrm{K}$ \\
\hline 1. Research \& Development areas need to be represented in & & & & & & & & & & & \\
\hline ERP & 0.1 & 0.1 & 0.1 & 0.1 & 0.1 & 0 & 0.1 & 0.1 & 0.1 & 0.1 & 0.1 \\
\hline 2. Need Project Planning / Scheduling & 0.2 & 0.2 & 0.2 & 0.2 & 0.2 & 0 & 0.2 & 0.2 & 0.2 & 0.2 & 0.2 \\
\hline 3. Need to account for time and material for federal projects & 0.18 & 0.18 & 0.18 & 0.18 & 0.18 & 0.09 & 0.18 & 0.18 & 0.18 & 0.18 & 0.18 \\
\hline $\begin{array}{l}\text { 4. Bill of Material - accommodating coding related to issues of } \\
\text { AS9100 }\end{array}$ & 0 & 0.105 & 0.14 & 0.14 & 0.14 & 0 & 0.14 & 0.14 & 0.14 & 0.14 & \\
\hline 5. Accommodate quoting & 0.16 & 0.16 & 0.16 & 0.16 & 0.16 & 0.16 & 0.16 & 0.16 & 0.16 & 0.16 & 0.16 \\
\hline 6. Emphasis on the importance to control documentations & 0.18 & 0.18 & 0.18 & 0.18 & 0.18 & 0.09 & 0.18 & 0.18 & 0.18 & 0.18 & 0.18 \\
\hline 7. System that easy to manage and administer & 0.18 & 0.18 & 0.18 & 0.18 & 0.18 & 0.18 & 0.18 & 0.18 & 0.18 & 0.18 & 0.18 \\
\hline 8. System needs to be easy to integrate & 0.16 & 0.16 & 0.16 & 0.16 & 0.16 & 0.16 & 0.16 & 0.16 & 0.16 & 0.16 & 0.16 \\
\hline 9. Dashboard (Integrated pictures of everything) & 0.16 & 0.16 & 0.16 & 0.16 & 0.16 & 0.16 & 0.08 & 0.16 & 0.16 & 0.16 & 0.16 \\
\hline 10. Track training hours & 0.12 & 0.12 & 0.12 & 0.06 & 0.12 & 0.06 & 0.12 & 0 & 0.12 & 0.12 & 0.12 \\
\hline 11. Automated Requisition System - Email notification. & & & & & & & & & & & \\
\hline Electronic Signatures & 0.04 & 0.04 & 0.04 & 0.04 & 0.04 & 0 & 0.04 & 0.04 & 0.04 & 0.04 & 0.04 \\
\hline 12. Bar Code Optional but desirable & 0.18 & 0.18 & 0.18 & 0.18 & 0.18 & 0.18 & 0.18 & 0.18 & 0.18 & 0.18 & 0.18 \\
\hline $\begin{array}{l}\text { 13. Multiple companies with same pages. CME may require } \\
\text { that another company may be setup in order to share }\end{array}$ & & & & & & & & & & & \\
\hline revenue/cost etc. & 0.08 & 0.08 & 0.08 & 0.08 & 0.08 & 0.08 & 0.08 & 0 & 0.08 & 0.08 & 0.08 \\
\hline 14. Track products station to station & 0.16 & 0.16 & 0.16 & 0.16 & 0.16 & 0.16 & 0.16 & 0.16 & 0.16 & 0.16 & 0.16 \\
\hline 15. Import/Export coding. & 0.18 & 0.18 & 0 & 0.18 & 0.18 & 0 & 0.18 & 0.18 & 0.18 & 0.18 & 0.18 \\
\hline $\begin{array}{l}\text { 16. Tracking a rating suppliers (List Preferred suppliers, } \\
\text { reporting late deliveries) }\end{array}$ & 0.14 & 0.14 & 0.14 & 0.14 & 0.14 & 0.14 & 0.14 & 0.14 & 0.14 & 0.14 & 0.14 \\
\hline $\begin{array}{l}\text { 17. Need to facture in the package ability to update Small } \\
\text { Systems to allow those minor reports, etc }\end{array}$ & 0 & 0.12 & 0 & 0.12 & 0.12 & 0 & 0.12 & 0 & 0.12 & 0.12 & 0.12 \\
\hline $\begin{array}{l}\text { 18. Permission to allow user control access to the system or to } \\
\text { areas of the system }\end{array}$ & 0.16 & 0.16 & 0.16 & 0.16 & 0.16 & 0.16 & 0.16 & 0.16 & 0.16 & 0.16 & 0.16 \\
\hline 19. Communication with ACT Software will be beneficial & 0 & 0.06 & 0 & 0.06 & 0.09 & 0.06 & 0.09 & 0.12 & 0 & 0 & 0.12 \\
\hline 20. Human Resource Functions & 0.06 & 0.06 & 0.06 & 0.03 & 0.06 & 0 & 0.03 & 0.06 & 0 & 0.03 & 0.03 \\
\hline
\end{tabular}

Figure 45 - Other Requirements performance score matrix

The performance matrix shows the scores for the evaluation of eleven candidate systems. The PCA technique should allow us to reduce the p-dimensional space, described by the variables, with each variable representing an orthogonal axis of that space, in terms of a $\mathrm{k}$-dimensional space where $\mathrm{k}<\mathrm{p}$. Another objective is to retain as much information as possible in the first three dimensions (factors) of this newly defined space, and therefore establish an ability to graphically represent the correlation of the combined variables, and significantly reduce the evaluation workload of the selection process. These combined variables are the factors produced by the PCA calculations. 
For these techniques to behave well, it is assumed that; there is a large ratio of observations to the variables, that the sample size is large enough to yield reliable estimates of the correlations between the variables, that there are identifiable linear relationships between the variables, there are no outliers in the data, and a modest degree of co-linearity exists between variables. In addition, it is assumed that the crosscorrelation between variables is because they are measuring the same attribute. In the example matrix there are $n=11$ subjects (i.e. the candidate vendors), and 20 variables (i.e. the requirements). Note: In the following data representations the matrix has been transposed to conform to general technique, and the long form descriptions of the requirements have been reduced to their index numbers.

In order to validate the results, a first step is to ensure that the inter-correlation matrix does not represent an identity matrix (suggesting a random sample) in which the variables are non-collinear. The software application XLStat ${ }^{\circledR}$, utilizes the Bartlett Sphericity Test to calculate the determinant of the sample matrix, and develop a Chi-square statistic which tests a "null" hypothesis, that the matrix is an identity matrix. If the value is significant then the null hypothesis is negated and the correlation matrix is analyzed as a non-random sample. For this sample these test results and for all other categories were sufficient to negate the null hypothesis. 
Bartlett's sphericity test:

Chi-square (Critical value)

223.160

DF

190

p-value

alpha

0.05

Test interpretation:

H0: There is no correlation significantly different

from 0 between the variables.

Ha: At least one of the correlations between the variables is significantly different from 0 .

As the computed $p$-value is lower than the significance level alpha $=0.05$, one should reject the null hypothesis $\mathrm{H} 0$, and accept the alternative hypothesis $\mathrm{Ha}$.

The risk to reject the null hypothesis $\mathrm{H} 0$ while it is true is lower than $0.01 \%$.

Figure 46 - Bartlett's Sphericity Test Results for Other Requirements

The initial results suggested that the twenty variables being tested in the performance matrix had a high degree of correlation, i.e. they were measuring the same attribute; and the PCA calculations suggested that the original twenty variables could be reduced to a total of nine combinatorial factors.

\begin{tabular}{|c|c|c|c|c|c|c|c|c|c|}
\hline $\begin{array}{l}\text { Principal } \\
\text { Component } \\
\text { Analysis: }\end{array}$ & & & & & & & & & \\
\hline \multicolumn{10}{|l|}{ Eigenvalues: } \\
\hline & F1 & $\mathrm{F} 2$ & F3 & F4 & F5 & F6 & $\mathrm{F} 7$ & $\mathrm{~F} 8$ & F9 \\
\hline Eigenvalue & 6.399 & 2.398 & 1.594 & 0.962 & 0.864 & 0.464 & 0.214 & 0.091 & 0.013 \\
\hline Variability (\%) & 49.221 & 18.447 & 12.264 & 7.400 & 6.649 & 3.570 & 1.646 & 0.703 & 0.100 \\
\hline Cumulative \% & 49.221 & 67.668 & 79.931 & 87.332 & 93.981 & 97.550 & 99.196 & 99.900 & 100.000 \\
\hline
\end{tabular}

Figure 47 - PCA Information for Other Requirements 
It can be seen from Figure 16 and Figure 17, that fully ninety seven percent of the variability, and therefore a very large proportion of the representative information, can be derived satisfactorily from only six of the factors.

Figures 17, below, shows the results developed for the "other requirements" data set in the PCA process using Pearson's (n) methodology. For all of the scree plots that follow, the line indicates the cumulative variability curve, and the columnar plot shows the individual eigenvalues associated with each of the factors.

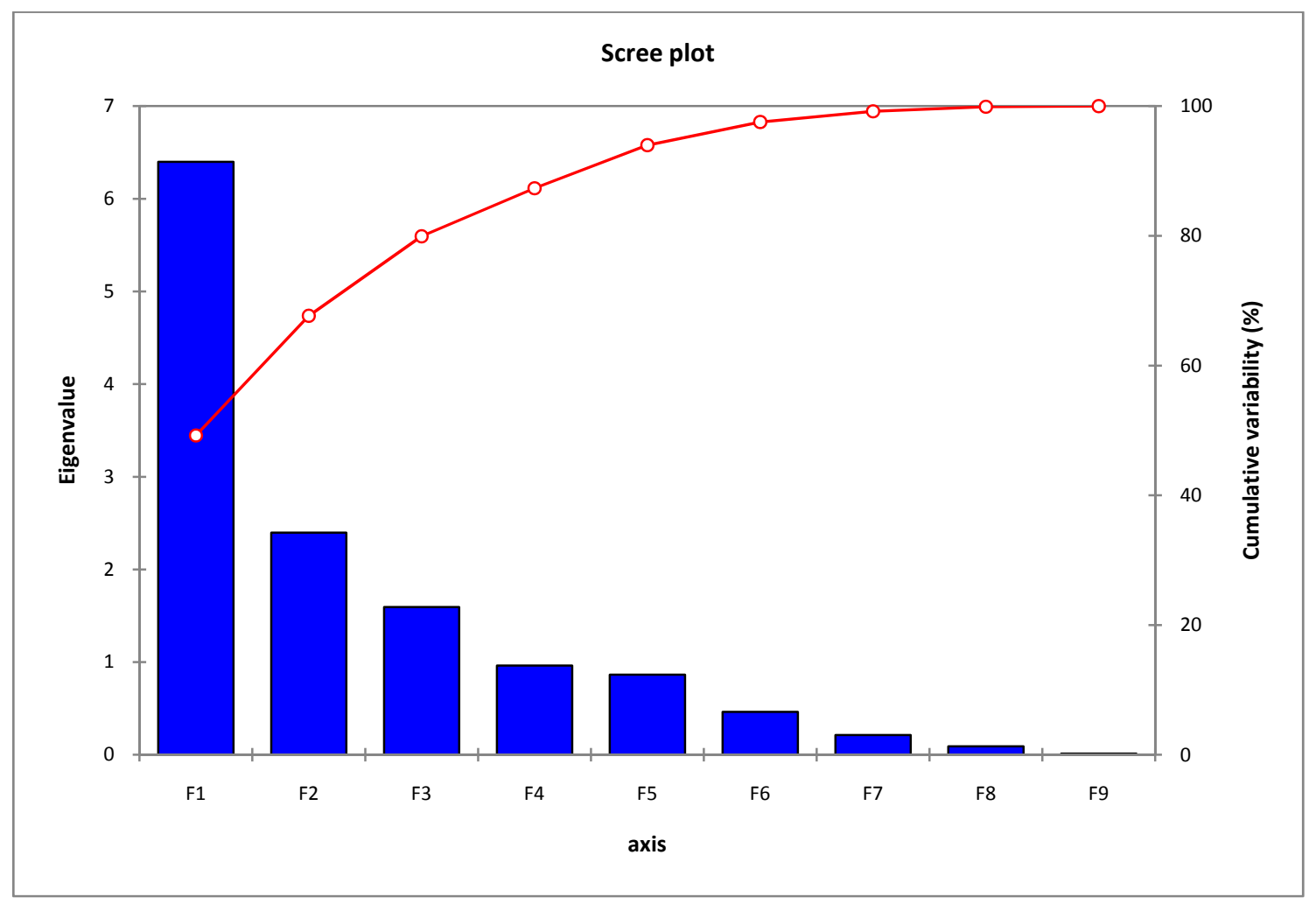

Figure 48 - Scree plot for Other Requirements

The scree plot (Cattell, 1966) illustrates this result; good standard practice in PCA interpretation suggests that factors with eigenvalues below 0.3 can be safely excluded 
from further analysis. These results suggest that factors 7,8 and 9 can safely be dropped and that we are likely to get substantially as accurate results with a smaller factored variable group in our future assessments.

From Figure 18 , it can be seen that Factor 1 (F1) has substantial contributions from six of the original variables, and more modest contributions to overall variability is provided by another three of the variable. The balance of the variables provides little contribution to F1's variability, and may indeed be good candidates for exclusion from future requirements lists. .

Correlations between variables and factors:

\begin{tabular}{|c|c|c|c|c|c|c|c|c|c|}
\hline & $\mathrm{F} 1$ & F2 & F3 & F4 & F5 & F6 & F7 & F8 & F9 \\
\hline 1 & 0.990 & -0.034 & -0.095 & -0.011 & -0.008 & -0.048 & -0.081 & -0.020 & 0.001 \\
\hline 2 & 0.990 & -0.034 & -0.095 & -0.011 & -0.008 & -0.048 & -0.081 & -0.020 & 0.001 \\
\hline 3 & 0.990 & -0.034 & -0.095 & -0.011 & -0.008 & -0.048 & -0.081 & -0.020 & 0.001 \\
\hline 4 & 0.553 & -0.125 & 0.119 & 0.738 & 0.191 & 0.271 & 0.086 & 0.034 & -0.040 \\
\hline 5 & 0.000 & 0.000 & 0.000 & 0.000 & 0.000 & 0.000 & 0.000 & 0.000 & 0.000 \\
\hline 6 & 0.990 & -0.034 & -0.095 & -0.011 & -0.008 & -0.048 & -0.081 & -0.020 & 0.001 \\
\hline 7 & 0.000 & 0.000 & 0.000 & 0.000 & 0.000 & 0.000 & 0.000 & 0.000 & 0.000 \\
\hline 8 & 0.000 & 0.000 & 0.000 & 0.000 & 0.000 & 0.000 & 0.000 & 0.000 & 0.000 \\
\hline 9 & -0.155 & -0.169 & -0.597 & -0.352 & 0.641 & 0.229 & -0.049 & 0.029 & -0.017 \\
\hline 10 & 0.333 & 0.854 & -0.170 & -0.132 & -0.241 & 0.074 & -0.011 & 0.220 & -0.021 \\
\hline 11 & 0.990 & -0.034 & -0.095 & -0.011 & -0.008 & -0.048 & -0.081 & -0.020 & 0.001 \\
\hline 12 & 0.000 & 0.000 & 0.000 & 0.000 & 0.000 & 0.000 & 0.000 & 0.000 & 0.000 \\
\hline 13 & -0.071 & 0.953 & -0.067 & -0.096 & -0.085 & 0.159 & 0.017 & -0.195 & -0.041 \\
\hline 14 & 0.000 & 0.000 & 0.000 & 0.000 & 0.000 & 0.000 & 0.000 & 0.000 & 0.000 \\
\hline 15 & 0.726 & -0.050 & 0.407 & -0.328 & 0.234 & -0.238 & 0.290 & 0.014 & -0.040 \\
\hline 16 & 0.000 & 0.000 & 0.000 & 0.000 & 0.000 & 0.000 & 0.000 & 0.000 & 0.000 \\
\hline 17 & 0.511 & 0.497 & 0.577 & -0.079 & 0.293 & 0.246 & 0.034 & 0.002 & 0.073 \\
\hline 18 & 0.000 & 0.000 & 0.000 & 0.000 & 0.000 & 0.000 & 0.000 & 0.000 & 0.000 \\
\hline 19 & 0.004 & -0.525 & 0.669 & -0.347 & -0.206 & 0.293 & -0.161 & 0.015 & -0.040 \\
\hline 20 & 0.514 & -0.428 & -0.446 & -0.177 & -0.411 & 0.305 & 0.244 & -0.024 & 0.024 \\
\hline
\end{tabular}

Figure 49 - Correlation Matrix for Other Requirements 
The special case of variables 5,7 and 8 is worthy of consideration. The evaluated performance chart, Figure 14, shows common valuation for each of these variables for all subject systems. These data suggest that the attributes that are proposed in these variables are likely to universally be present in all systems. This result should be tested further.

For the forty five manufacturing related variables, there was a significant reduction in the number of factors compared to the original variable set. In fact, there are only eight factors required to address one hundred percent of the variability in the data. Factor F8 falls below the standard test value for its characteristic eigenvalues $(0.3)$, below which the factor is not making a sufficient contribution to the variability to be retained. Therefore, the forty-five requirements gathered within the manufacturing category, could be replaced by seven combined factors in future selection processes.

\section{Principal Component \\ Analysis:}

Eigenvalues:

\begin{tabular}{lllllllll}
\hline & F1 & F2 & F3 & F4 & F5 & F6 & F7 & F8 \\
\hline Eigenvalue & 8.217 & 4.081 & 2.525 & 1.894 & 1.178 & 0.651 & 0.378 & 0.076 \\
Variability (\%) & 43.248 & 21.481 & 13.287 & 9.970 & 6.199 & 3.428 & 1.988 & 0.398 \\
Cumulative \% & 43.248 & 64.729 & 78.016 & 87.987 & 94.186 & 97.614 & 99.602 & 100.00 \\
\hline
\end{tabular}

Figure 50 - PCA Information for Manufacturing Requirements 
For the manufacturing category of the requirements, a substantial proportion of the test variability $(99.6 \%)$ is attributable to seven of the suggested eight factors.

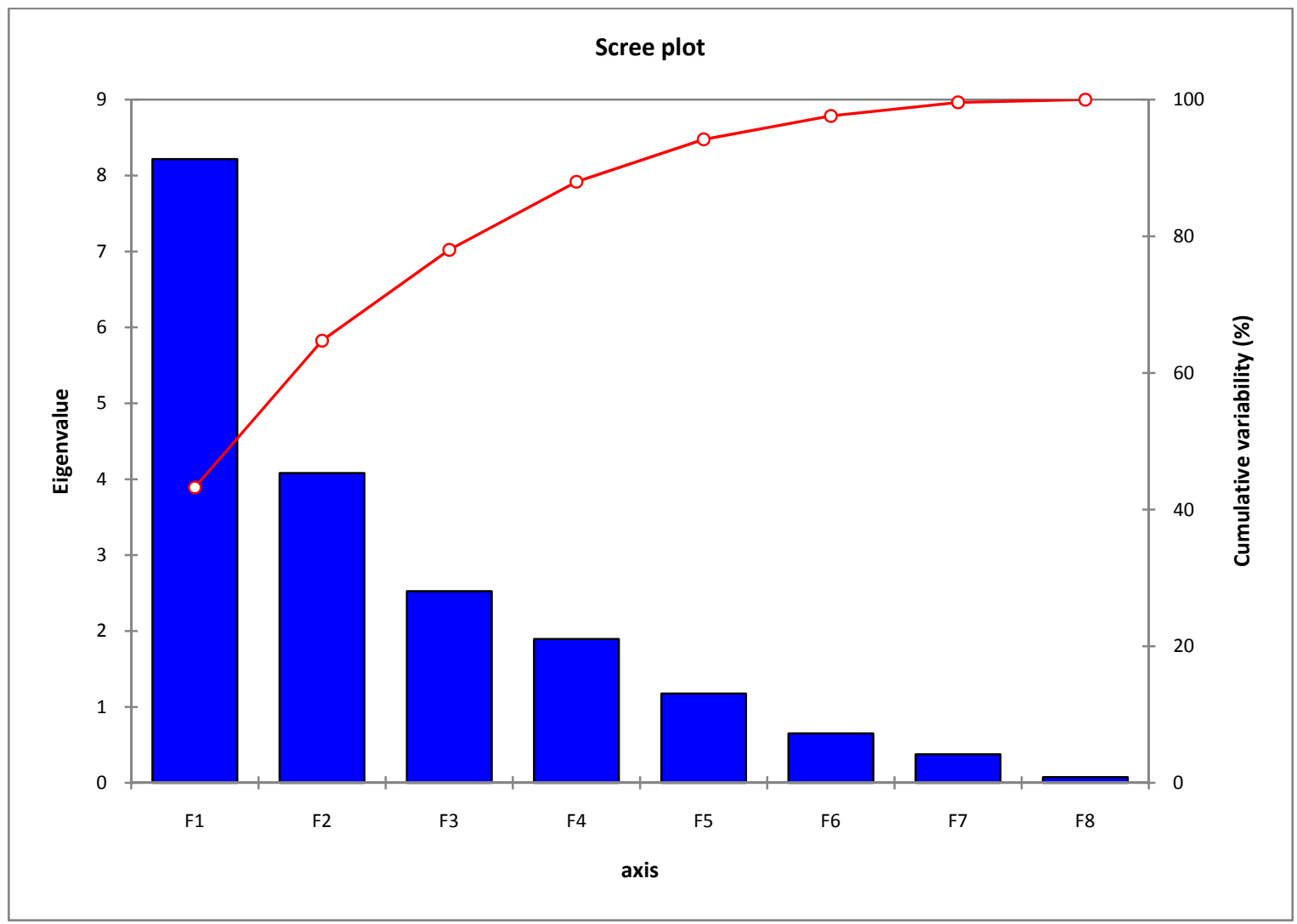

Figure 51 - Scree plot for Manufacturing Requirements

The accounting requirements gathering process identified twenty nine individual requirements. PCA analysis of the fully completed performance assessment showed that the accounting variables could be substituted by ten factors. 
Principal Component Analysis:

Eigenvalues:

\begin{tabular}{lllllllllll}
\hline & F1 & F2 & F3 & F4 & F5 & F6 & F7 & F8 & F9 & F10 \\
\hline Eigenvalue & 8.419 & 5.038 & 2.282 & 1.648 & 1.089 & 0.627 & 0.452 & 0.242 & 0.119 & 0.084 \\
Variability (\%) & 42.093 & 25.191 & 11.411 & 8.241 & 5.445 & 3.136 & 2.261 & 1.209 & 0.595 & 0.418 \\
Cumulative \% & 42.093 & 67.283 & 78.694 & 86.936 & 92.381 & 95.517 & 97.778 & 98.987 & 99.582 & 100.000 \\
\hline
\end{tabular}

Figure 52 - PCA Information for Accounting Requirements

In this analysis, it was also noted that the final three factors proposed by the PCA again fell below the threshold eigenvalues.

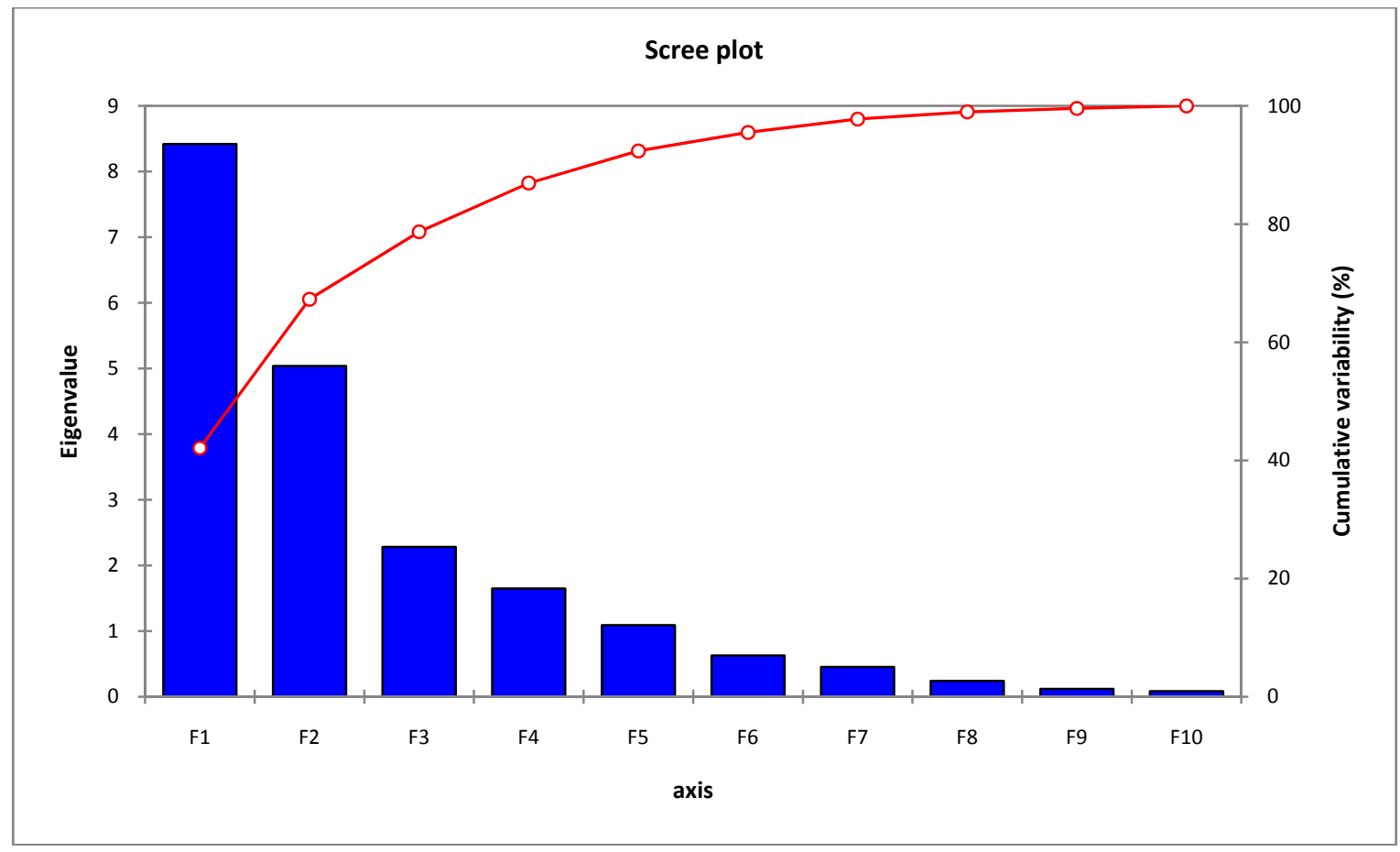

Figure 53 - Scree plot for Accounting Requirements.

For the accounting requirements, the PCA showed that $97.77 \%$ of the variability in the data was contained in the first seven factors, 


\section{Principal Component Analysis:}

Eigenvalues:

\begin{tabular}{llllll}
\hline & F1 & F2 & F3 & F4 & F5 \\
\hline Eigenvalue & 6.156 & 2.260 & 1.588 & 0.797 & 0.200 \\
Variability (\%) & 55.961 & 20.541 & 14.438 & 7.242 & 1.818 \\
Cumulative \% & 55.961 & 76.502 & 90.940 & 98.182 & 100.00 \\
\hline
\end{tabular}

Figure 54 - PCA Information for Reporting Requirements

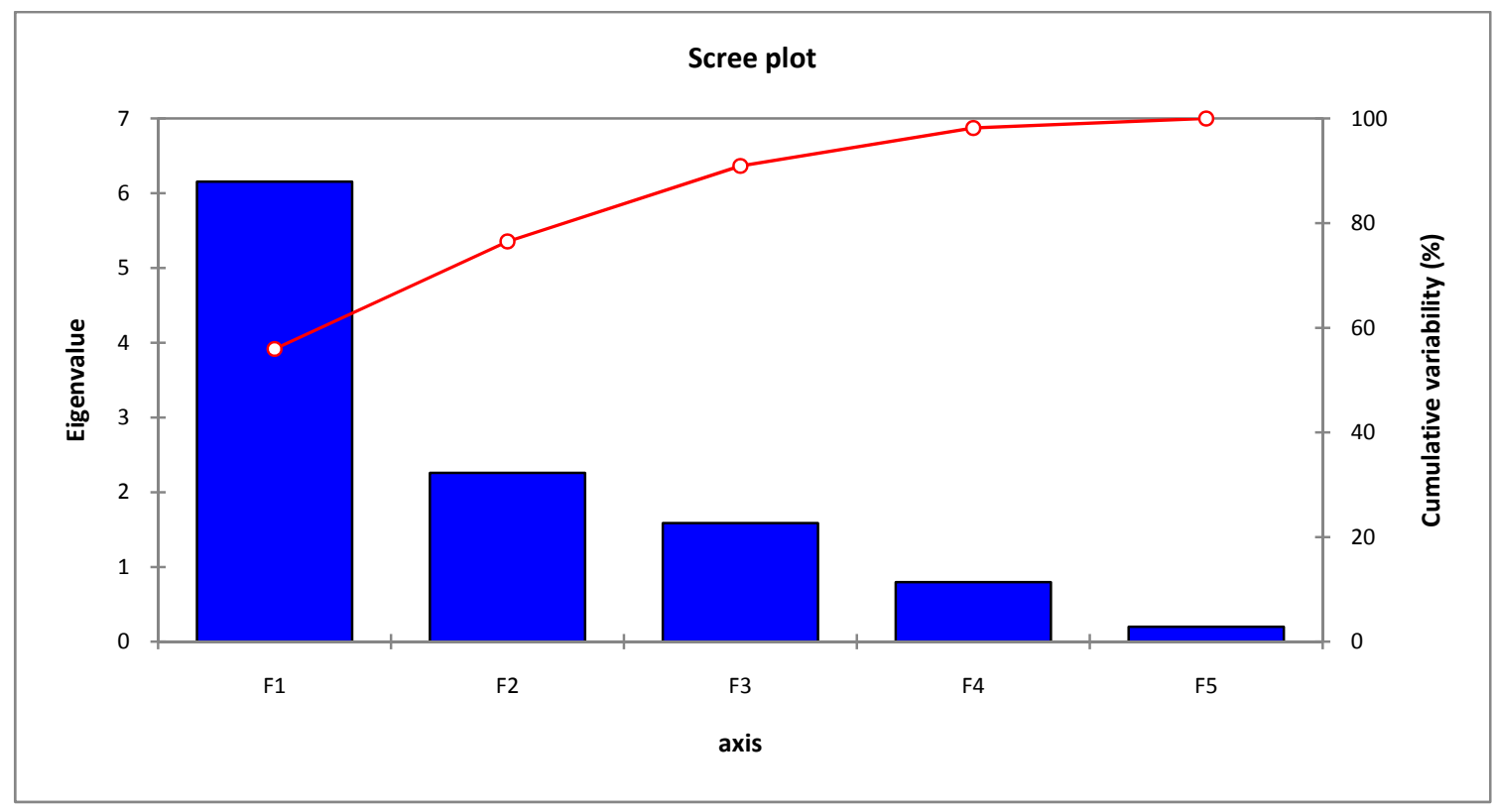

Figure 55 - Scree plot for Reporting Requirements

The final component of the requirements set was assessed using the PCA procedure.

The results suggested that, twenty three requirements associated with reporting may be replaced by five composite factors with $100 \%$ of the variability retained; or by four factors if it is acceptable to only retain $98.182 \%$ of the variability. 


\subsection{Expert Validation}

Subsequent to the development and testing of the methodology, industry experts were identified to evaluate and validate the general applicability and level of effectiveness of the methodology to small and medium-sized businesses. A short survey was conducted to determine if the methodology met its purpose. Five industry experts responded to the survey:

Name: Liping Li Ph.D.

Position/Job Title: Senior Consultant - SAP Implementation

Experience/Background/Credentials:

4 years of SAP Business One Implementation, Ph.D. ISE

Name: Alfred Menendez

Position/Job Title: CEO -Menendez and Associates

Experience/Background/Credentials:

15 Years - Business Owner, ERP Implementation and design of business computer systems; MBA, BS Computer Science.

10 Years - Business software development and sales.

Name: George Schulte

Position/Job Title: Senior Accounting Manager - NGC.

Experience/Background/Credentials: 
24 Years NGC - accounting, implementation team manager for SAP ERP

Implementation, ERP Implementation support manager and consultant for suppliers. BA Accounting, CPA.

Name: Alfredo Moran Hassan

Position/Job Title: CEO - Epic Consultants

Experience/Background/Credentials: Have participated in two ERP Implementations $(\mathrm{BaaN})$

Name: Walter Quintero

Position/Job Title: Research Analyst and IT Project Manager

Experience/Background/Credentials:

B.Sc. Computer Science

Over 12 years of experience in creating and maintaining information technology project plans that deal with task, milestones dates, project status and resource allocation. Computer Engineer background with vast experience in custom system implementation using software life-cycle methodology. In the last 5 years, Mr. Quintero has assisted with several evaluation and implementation of ERP Projects that involved hundreds of ERP System for small business. Successfully implemented Sage and MISys ERP Systems, among others, where businesses experience a reduction in operating cost and increased profits. 
The survey results indicated a broad acceptance of the methodology and verified that the methodology successfully addresses its purpose.

Four experts strongly agreed, and one agreed that - the methodology was sufficient to assist SME's in selecting an ERP System. All experts strongly agreed that - the strategic review/chartering process was likely to orient the selection team to the company's business objectives, and that the joint requirements planning process was sufficient to capture the company's requirements.

Three experts agreed and two strongly agreed that the business process review and update process was sufficient to orient the selection team to the company's business methods.

Three experts strongly agreed, and two agreed that the ERP System identification process was likely to identify a broad range of vendors; and that, the phased selection process adopted by the methodology was likely to provide sufficient information to achieve the purpose. 


\begin{tabular}{|c|c|c|c|c|c|c|c|c|}
\hline & & A. Menendez & Liping Li Ph.D. & G. Schulte & A. Moran Hassan & W. Quintero & Mean & Mode \\
\hline 1 & $\begin{array}{l}\text { Is the methodology sufficient to assist SME's in the selection of } \\
\text { an appropriate ERP system? }\end{array}$ & 4 & 3 & 4 & 4 & 4 & 3.8 & 4 \\
\hline 2 & $\begin{array}{l}\text { Does the strategic review/chartering process orient the selection } \\
\text { team to the company's objectives? }\end{array}$ & 4 & 4 & 4 & 4 & 4 & 4 & 4 \\
\hline 3 & $\begin{array}{l}\text { Does the Joint Requirements Planning process serve to } \\
\text { sufficiently capture the company's requirements? }\end{array}$ & 4 & 4 & 4 & 4 & 4 & 4 & 4 \\
\hline 4 & $\begin{array}{l}\text { Does the Business Process review and update process serve to } \\
\text { orient the selection team to the company's business methods? }\end{array}$ & 4 & 3 & 3 & 3 & 4 & 3.4 & 3 \\
\hline 5 & $\begin{array}{l}\text { Does the ERP system identification process serve to identify the } \\
\text { broad range of vendors? }\end{array}$ & 3 & 3 & 4 & 4 & 4 & 3.6 & 4 \\
\hline 6 & $\begin{array}{l}\text { Does the phased selection process - RFI, performance } \\
\text { demonstration, RFP, cost evaluation, final selection provide } \\
\text { sufficient information? }\end{array}$ & 3 & 4 & 3 & 4 & 4 & 3.6 & 4 \\
\hline & $\begin{array}{l}\text { Evaluation Scale: } \\
\text { Strongly Agree } 4 \text {; Agree 3; Disagree 2; Strongly Disagree } 1\end{array}$ & & & & & & & \\
\hline
\end{tabular}

Figure 56 - Methodology Validation Survey 


\section{CONCLUSIONS}

\subsection{Summary}

We rigorously applied Thomann's meta-methodology to the problem of developing a methodology for the selection of an ERP system by a SME. The development process for the new methodology gained significant benefit from an industrial survey of similar organizations which had recently selected and implemented ERP systems. We then successfully employed the new methodology in a case study which sought to select an ERP system for the ABC Company. The primary benefit of the methodology is that we have imposed both structure and discipline to the process of selecting an ERP system for an SME; taking the largely ad hoc and unstructured general practice and introducing a framework and pathway to a logical outcome. The methodology provides an objective selection outcome, without the commercial bias that is frequently introduced by industrial methods that are currently in use.

The methodology developed in this paper is a tool designed to inform and help decision makers at SME's and their consultants in making selections from a complex pool of alternatives in which qualitative and quantitative variables must be balanced. The tool that has been developed allows collaboration between decision makers and participants with varying degrees of knowledge and from all areas of the company, and integrates their inputs in an effective way. 
The final selection process lends itself to easy explanation to the final decision maker, and provides supporting data in a spreadsheet format to enhance the acceptance of the proposed solution. The methodology utilizes readily understandable process logic.

The research shows that there is a powerful linkage between a strong methodological approach to the ERP selection process and that companies that follow this principle appear to have higher than average ERP implementation success.

The research further shows that, the integration and use of systems engineering methods and techniques, within a well executed methodology can serve to ease the process of ERP system selection, and assist small and medium-sized enterprises identify usable systems at lower risk than is currently the case.

The methodology was assessed by a group of industry experts, and it was verified with high concurrence that the methodology serves its purpose, and that the procedural elements proposed are sufficient and likely to be successful in actual practice.

An effective ERP system selection process depends on full participation of the small business managers, administrative and technical staff. The key to a good selection outcome is fully understanding the requirements of the company, an ability to project an accurate understanding of how work will be done in the future and the willingness to work through the 'revolution resistance' that is naturally developed in the face of change. 
The selection of an ERP system is a process in which a faulty conclusion poses a significant risk of business failure to small businesses. Recent studies, although few in number, have begun to focus on the selection of an ERP system as a high value-added exercise, which can make the difference between a difficult implementation and a smoother one (Verville, 2003). The ultimate test of success of an ERP system is whether it succeeds in producing the strategic benefits that were defined in the planning stage of the acquisition process.

The methodology proposed in this paper suggests that making a determination of the utility value of various choices, with respect to the engineering requirements, is likely to yield a small group of candidate options, that may be assessed against the common framework of how well they address those requirements, and that the further step of economic analysis is required to determine which system represents the best value option.

The methodology utilized to facilitate the selection process at $\mathrm{ABC}$ was used as a developmental tool in conformance to the meta-methodology. Based on analysis of the case study, the data suggest that the large number of variables represented by the 147 individual requirements can be effectively reduced to approximately twenty eight composite factors with a high expectation that the derived conclusion will include the vast majority of the variability in the long form review. In terms of individual workload, always a key concern at a small business, this reduction in the overall number of evaluation data points may provide a significant practical benefit to future selection 
processes, and future studies should test the validity of this process against other classes of small business.

\subsection{Future Research}

Future research should investigate in greater detail the application of methodological approaches associated with supplier selection and evaluation for a broader range of services and software, and additional research into the purchasing practices of small firms is clearly needed.

This study has combined both case study experience and used survey techniques to test the assumptions of success as they apply to small businesses; it would be instructive for additional future surveys and case studies to be conducted, to establish whether more general patterns of success and failure factors can be identified.

The methodology developed in this study is designed to accommodate a broad range of small and medium-sized businesses that would benefit by the development of a comprehensive data resource that will facilitate the selection process by encompassing all the ERP system data required to make a successful selection. Such a data resource would dramatically reduce the workload and burden of the data gathering activities of the methodology.

$\mathrm{ABC}$ and its contemporaries, have all developed forward looking and innovative ways to extract value from the process changes driven by ERP introduction. While this is a 
testament to the adaptability and strong ability of these companies to absorb and react to change, a characteristic of successful small businesses, it is also a reflection of the high levels of executive commitment to achieving project success. A crucial element of the overall success of an ERP implementation is the assignment the right resources; internal staff, external consultants, to the project. Future research may explore the dynamics of these relationships with regard to the successful outcome of ERP implementation.

Equally important are the project reporting process and the nature of the metrics for testing progress and success that are adopted during the implementation project. Both the experiences with $\mathrm{ABC}$ and the survey data support the conclusion that business process reengineering efforts need to initiated and substantially completed before the ERP system is deployed, or even selected. There is clearly an element of recursion and iteration regarding these efforts, but a forward looking company will have analyzed and identified BPR needs as part of the same decision process by which the need for an ERP was identified. The potential benefits of re-engineered processes will have been independently evaluated and the implementation of BPR will have standalone returns on investment. By initiating BPR early, the baseline by which the ERP selection is made will have been firmly grounded in the preferred way of doing business. In other words the ERP selection requirements will be derivative of the reengineered processes.

ERP selection should occur in conjunction with an assessment of the hardware and IT needs of the company. Major benefits can be derived from configuring hardware and IT systems to allow seamless integration of new software. 
The earlier in the implementation project these upgrades are assessed and identified, the smoother the implementation will be. Equally important, is the necessity to identify the breadth of training needs that exist in the enterprise early on in the requirements engineering process; and consideration should be given to identifying how to address any skill deficiencies, whether through training, or by hiring new employees. The essential conditions for success also include good communications, which will allow for fewer unanticipated functional delays, greater buy-in at all levels of the company, and allows the encouragement of effective involvement. The primary outcome of good communication strategies is to develop strong comprehensively involved, well informed teams, who can intervene proactively to reduce functional stalemates which can increase the costs of implementation.

In addition, good project management is crucial to creating the structure by which the implementation is governed; a strong project manager can facilitate and direct the path of the implementation, identify the critical risk loaded processes, and mitigate or manage the outcomes. The authority, and demeanor of the project lead is vital to success, in all the survey cases the company's had been able to identify strong leaders, with institutional credibility to lead their projects. The benefit of having leaders of this profile is that, while resistance to change cannot be completely put aside, and organizational politics are ever present as realities of the workplace, a well-respected manager with senior management's clear and unwavering support, can ease the transition. Contrary to earlier findings, for this class of small businesses at least, the availability of good project 
managers within the organization was high and in addition the in-house BPR knowledge base was strong (Muscatello, J. R., Small, M.H., \& Chen, I.J., 2003) (Yen, H.R., \& Sheu,C., 2004). We suggest that researchers revisit this issue to determine if the findings of this study are supported in the new business environment, where so many former employees of large enterprises are now small and medium-sized business employees. 


\section{LIST OF REFERENCES}

Al-Mashari, M. (2002). Implementing ERP through SAP R/3: A process change management (PCM) perspective. King Saud University Journal: Computer and Information Sciences Division., 16, 34-45.

Al-Mashari, M. (2003). Enterprise resource planning (ERP) systems: a research agenda. Industrial Management \& Data Systems, 165-170.

Al-Mashari, M., \& Zairi, M. (2000). Supply-chain re-engineering using enterprise resource planning (ERP) systems:an analysis of a SAP R/3 implementation case. International Journal of Physical Distribution and Logistics Management, 30(3/4), 296-313.

Al-Mudimigh, A., Zairi, M., \& Al-Mashari, M. (2001). ERP software implementation: An integrative framework. European Journal of Information Systems, 10(4), 216226.

Andriole, S. (2006). The collaborate/integrate business technology strategy.

Communications of the ACM (pp. 85-99). Association for Computing Machinery.

APICS. (2008). APICS Dictionary (12th ed.). APICS.

Babey, E. (2006). Costs of enterprise planning system implementation. New Directions for Higher Education, 136, 21-33.

Bakås O., R. A. (2007). Holistic ERP selection methodology. Proceedings of the 14th International EurOMA Conference "Managing Operations in an Expanding Europe. Ankara: SINTEF.

Bernroider, E. K. (2001). ERP selection process in midsize and large organizations. Business Process Management, 7(3), 251-257.

Bernroider, E.W.N., Stix, V. (2006). Profile distance method - a multi-attribute decision making approach for information system investments. Decision Support Systems, 42, 988-998.

Boudreau, M.C.,\& Robey, D. (1999). Organizational transition to enterprise resouce planning systems:Theoretical choices for process research. Proceedings of the 20th International conference on Information Systems, (pp. 291-299). Charlotte.

Brehm, L. H. (2001). Tailoring ERP systems: A spectrum of choices and their implications. Proceedings of the 34th Annual Hawaii International Conference on System Sciences. Maui: IEEE Press. 
Brehm, L., Heinzl, A., \& Markus, M. (2001). Tailoring ERP Systems: A Spectrum of Choices and their Implications. Proceedings of the 34th Hawaii International Conference on System Sciences, (pp. 1-9).

Buss, M. (1983, January- February). How to Rank Computer Projects. Harvard Business Review, pp. 118-124.

Capaldo, G., Iandoli, L., Rippa, P., Mercanti, S., \& Troccoli, G. (2008). An AHP approach to evaluate factors affecting ERP Implementation Success. Proceedings of the World Congress on Engineering and Computer Science 2008. San Francisco, USA.

Cattell, R. B. (1966). The Scree Test for the Number of Factors. Multivariate Behavioural Research, 245-276.

Cebeci, U. (2009). Fuzzy AHP-based decision support system for selecting ERP systems in teextile industry by using balanced scorecard. Expert Systems with Applications, 36, 8900-8909.

Chang, M., \& et al. (2008). Understanding ERP system adoption from the user's perspective. International Journal of Production Economics, 113, 928-942.

Chen, I. (2001). Planning for ERP systems:Analysis and future trends. Busness Process Management Journal, 7(5), 374-386.

Chen, Y. (2011). Structured methodology for supplier selection and evaluation in a supply chain. Information Sciences, 1651-1670.

Clemen, R. (1996). Making Hard Decisions: An Introduction to Decision Analysis. Pacific Grove: Duxbury Press.

Correa, S. (2007). The process of enterprise alignment in an ERP project: A view from the trenches. In M. Rodenes, \& R. Hackney (Ed.), CD-ROM/Online Proceedings of the European and Mediterranean Conference on Information Systems (EMCIS).

Crandall, R. C. (2005, January). The Life Cycles of Management Programs. APICS ThePerformance Advantage, pp. 36-41.

Cronbach, L. (1951). Coefficient alpha and the internal structure of tests. Psychometrika, 297-334.

Davenport, T. (1998, January-February). Putting the enterprise into the enterprise system. Harvard Business Review, pp. 16-17. 
Davenport, T. (2000). Enterprise systems and the supply chain. Journal of Enterprise Information Management, 17(1), 8-19.

Davenport, T. H. (1993). Process Innovation: Reengineering Work through Information Technology. Boston: Harvard Business School Press.

Deming, W. E. (1986). Out of Crisis. Boston: MIT Press.

El Sawah, S., Tharwat, A., \& Rasmy, M. (2008). A quantitative model to predict the Egyptian ERP implementation success index. Business Process Management Journal, 14(3), 288-306.

Evangelista, P. (1998). ERP Systems Strategies. American Production and Inventory Control Society Proceedings.

Ferman, J. (1999). Strategies for successful ERP connections. Manufacturing Engineering, 123(4), 48-60.

Gefen, D. (2002). Nurturing client's trust to encourage engagement success during the customization of ERP systems. Omega, 30(4).

Glass, R. (1999). Enterprise resource planning:Breakthrough and/or term problems. Database for Advances in Information Systems, 29(2), 14-16.

Gliem, J. G. (2003). Calculating, Interpreting, and Reporting Cronbach's Alpha Reliability Coeeficient for Likert Type Scales. Midwest Research to Practice Conference, (pp. 82-88).

Guha, S. (1997). Business Process Change and Organizational Performance: Exploring an Antecedent Model. Journal of Management Information Systems, 14(1), 119154.

Gupta, A. (2000). Enterprise Resource Planning: The emerging organizational value system. Industrial Management and Data Systems., 100(3), 114-118.

Gyampah, K., \& Salam, A. (2004). An extension of the technology acceptance model in an ERP implementation environment. Information and Management, 41, 731-745.

Hallikainen, P., Laukkanen,S. \& Sarpola, S. (2004). Reasons for ERP acquisition. International conference on Enterprise Information Systems. Porto.

Hammer, M. C. (1993). Reengineering the Corporation. New York: Harper Collins.

Hill, S. (1997). The wait is over. Manufacturing Systems, 15(6), 11-14. 
Holland, C.P., \& Light, B. (1999). A critical success factors model for ERP implementation. IEEE Software, 16(3), 30-36.

Holt, G. (1998). Which Contractor Selection Methodology? International Journal of Project Management, 16(3), 153-164.

Hong, K., \& Kim, Y. (2002). The critical success factors for ERP implementation. Information and Management, 40, 25-40.

Hutchinson, T. (1984). Meta-methodology. In Instructional development:The State of the $\operatorname{Art}$ (Vol. II).

Jacobs, F., \& Bendoly, E. (2003). ERP: Developments and directions for operations management research. European Journal of Operational Research, 146, 233-240.

Jacobs, F., \& Weston, F. (2007). ERP: A Brief History. Journal of Operations Management, 25, 357-363.

Jun, W. (2008). Critical Success Factors for ERP System Implementation. In Research and Practical Issues of Enterprise Information Systems II Volume 1 (Vol. 254, pp. 739-745). Boston: Springer.

Kaplan, R. (1992). The Balanced Scorecard: Measures that drive performance. Harvard Business Review, 1, pp. 71-79.

Kaplan, R. N. (1996). The balanced scorecard: Translating strategy into action. Boston: Harvard Business School Press.

Karaarslan, N., \& Gundogar, E. (2009). An application for modular capability-based ERP software selection using AHP method. Int $J$ Adv. Manuf. Technology, 42, 10251033.

Karsak, E.E.,Ozogul, C.O. (2009). An integrated decision making approach for ERP system selection. Expert Systems with Applications, 36, 660-667.

Kneppelt, L. R. (1974). A simple distributed systems approach to manufacturing information systems. AFIPS Joint Computer Conferences; Proceedings of the May 6-10 1974, national computer conference and exposition (pp. 485-490). Chicago: ACM.

Krause, D.R., Pagell,M., \& Curkovic,S. (2001). Toward a measure of competitive priorities for purchasing. Journal of Operations Management., 19, 497-512. 
Kumar, V. M. (2003). An investigation of critical management issues in ERP implementation: emperical evidence from Canadian organizations. Technovation, 23(10), 793-807.

Kumar, V., Maheshwari, B., \& Kumar, U. (2002). Enterprise resource planning systems adoption process: a survey of Canadian organizations. Int J Prod res, 40, 509-523.

Kuruppuarachchi, P. S. (2002). IT project implementation strategiesfor effective changes:A critical review. Logistics Information Management, 15(2), 126-137.

Lakatos, I. (1971). History of Science and its Rational Reconstructions. Boston Studies in the Philosophy of Science, 8, 91-135.

Lee, J.W., Kim, S.H. (2000). Using analytic network process and goal programming for interdependent information systems. Computers and Operations Research, 27, 367-382.

Liao,X.,Li, Y., Lu,B. (2007). A model for selecting an ERP system based on linguistic information processing. Information Systems, 32, 1005-1017.

Lien, C-T., Liang, S-K. (2005). An ERP selection model with project management viewpoint - A Fuzzy Multi-Criteria Decision-Making Approach. International Journal of the Information Systems for Logistics and Management, 1(1), 39-46.

Likert, R. (1932). A Technique for the Measurement of Attitudes. Archives of Psychology, 140, 1-55.

Lim, S. (2006). Forecasting of ERP Implementation Using Case-Based Reasonings. IJCSNS International Journal of Computer Science and Network Security, 6(4), 912.

Lucas, H.C., Moore,J.R. (1976). A multiple-criterion scoring approach to information system project selection. Information, 14(1), 1-12.

Luo, W., \& Strong, D. (2004). A Framework for Evaluating ERP Implementation Choices. IEEE Transactions On Engineering Management, 51(3), 322-333.

Mabert, V. A., Soni, A., \& Venkataramanan, M. A. (2001). Enterprise resource planning survey of U.S. manufacturing firms. Production and Inventory Management Journal, 52.

Madapusi, A., \& Ortiz, D. (2009). An empirical assessment of the impact of ERP TaskTechnology fit on decision quality. SWDSI proceedings, (pp. 102-107). 
Markus,M.L., \& Tanis,C. (2000). Framing the domains of IT research:Glimpsing the future throughthe past. In The enterprise systems experience--From adoption to success (pp. 173-207). Cincinnatti: Pinnaflex Educational Resources.

Martin, M. (1998). Enterprise resource planning. Fortune 137, pp. 149-151.

McCall, J., Richards, P.,and Walters, G. (1977). Factors in Software Quality. NTIS .

Millman, G. (2004, May). What did you get from ERP and what can you get? Financial Executive, pp. 38-42.

Mintzberg, H. (1979). The structuring of organizations: A synthesis of the Research. Englewood Cliffs, NJ: Prentice-Hall.

Morton, N. A., \& Hu, Q. (2008). Implications of the fit between organizational structure and ERP:A structural contingency theory perspective. International Journal of Information Management 28, 391-402.

Motwani, J. (2005). Critical factors for successful ERP implementation: Exploratory findings from four case studies. Computers in Industry, 56, 529-544.

Motwani, J. M. (2002). Successful implementation of ERP projects: Evidence from two case studies. International Journal of Production Economics., 75(1-2), 83-96.

Murphy, K., \& Simon, S. (2002). Intangible benefits valuation in ERP projects. Information Systems Journal, 12, 301-320.

Muscatello, J. (1999). ERP and its effects on the supply chain. APICS Manufacturing Symposium.

Muscatello, J. (2002). The potential use of knowledge management for training: A review and direction of future research. Business Process Management Journal, 9(3), $382-394$.

Muscatello, J. R., Small, M.H., \& Chen, I.J.,. (2003). Implementing enterprise resource planning (ERP) systems in small and midsize manuafcturing firms. International Journal of Operations and Production Management., 23(8), 850-871.

Muscatello,J.R., Small, M. H., \& Chen, I.J.,. (2003). Implementing enterprise resource planning (ERP) systems in small and mid-size manufacturing firms. International Journal of Operations and Production Management, 23(8), 850-871.

Nah, F. \&. (2001). Critical factors for successful iimplementation of enterprise systems. Business Process Managemnt Journal, 7(3), 285-296. 
Olson, D.L., Chae,B., \& Sheu, C. (2005). Issues in multinational ERP implementations. International Journal of Services and Operations Management, 1(1), 7-21.

Orlicky, J. (1969). The successful computer system: its planning, development, and management in a business enterprise. McGraw-Hill.

Orlicky, J. (1975). Material requirements planning: the new way of life in production and inventory management. McGraw-Hill.

Parthasarathy, S., \& Anbazhagan, N. (2007). Evaluating ERP Implementation choices using AHP. Int J of Enterprise Information Systems, 3.

Paulraj, A. \&. (2005). Strategic Supply Management: Theory and Practice. International Journal of Integrated Supply Management, 1(4), 457-477.

Percin, S. (2008). Using the ANP approiach in selecting and benchmarking ERP systems. Benchmarking:An international Journal, 15(5), 630-649.

Proia, A. (1984). DBMS checklist for manufacturing systems. Computerworld, 18(41), 63,81 .

Rao, S. (2000). Enterprise Resouce Planning: Business Needs and Technologies. Industrial Management and Data Systems, 100(2), 81-88.

Ravi Shankar, V. T. (2005). Analyzing alternatives in reserve logistics for end-of-life computers: ANP and balanaced scorecard approach. Computers and industrial Engineering, 48, 327-356.

Saaty, T. (1980). The Analytic Hierarchy Process. New York: McGraw-Hill.

Saaty, T. (1999). The Fundamentals of the Analytic Network Process. Kobe, Japan: ISAHP.

Santhanam, R., Kyparasis, G.J. (1995). A decision model for information system project selection. Computers and Operations Research, 22(8), 807-818.

Sarkar, H. (1980). Imre Lakatos' Meta-Methodology: An Appraisal. Philosophy of the Social Sciences, 397-416.

Sawyer, S. (2000). Packaged Software: Implications of the differences from custom approaches to software development. European Journal of Information Systems, 9, 47-58. 
Schniederjans, M.J., Wilson, R.L. (1991). Using the analytic hierachy process and goal programming for information system project selection. Information and Management, 20, 333-342.

Shang, S., \& Wu, T. (2005). A Model for Analyzing Organizational Performance of ERP Systems from a Resource-Based View. PACIS - Pacific Asia Conference on Information Systems.

Shewhart, W. (1939). Statistical Method from the Viewpoint of Quality Control. Washington, DC: The Graduate School, USDA.

Soh, C., Sia, S., Boh, W., \& Tang, M. (2003). Misalignments in ERP Implementation:A Dialectic Perspective. Int J of Human-Computer Interaction, 16(1), 81-100.

Taskin-Gumus, A. C. (2010). A Fuzzy-Analytic Network Process Based Approach For Enterprise Information System Selection. Journal of Engineering and Natural Sciences, 74-85.

Teltumbde, A. (2000). A framework for evaluating ERP projects. International Journal of Production Research, 38(17), 4507-4520.

Themistocleous, M., Irani, Z., O’Keefe, R., \& Paul, R. (2001). ERP Problems and Application Integration Issues:An Empirical Survey. Proceedings of the 34th Hawaii International Conference on System Sciences (pp. 1-10). IEEE.

Thomann, J. (1973). Meta-methodology: An overview of What it is and How it was Developed. Proceedigs of the Annual Convention of American Educational Research Association.

Trepper, C. (1999). ERP project management is key to a successful implementation. Retrieved from www.erphub.com

Triandis, H. C. (1971). Attitudes, and Attitude Change. Wiley \& Sons Inc.

Triandis, H. C. (1980). Values, Attitudes, and Interpersonal Behavior. 1979 Nebraska Symposium on Motivation: Beliefs, Attitudes, and Values (pp. 195-259). University of Nebraska Press.

Uta, A.,Intorsureanu,I., Mihalca,R. (2007). Criteria for the selection of ERP software. Informatica Economica, 42(2), 63-66.

Vaughan, C. (2001). System Implementation Success Factors;It's not just the Technology. College and University Machine Records Conference to CUMRECA Higher Education Administrative Technology Conference. 
Velcu, O. (2007). Exploring the effects of ERP systems on organizational performance. Industrial Management \& Data Systems, 107(9), 1316-1334.

Verville, J. (2003). A six-stage model of the buying process for ERP software. Industrial Marketing Management, 32, 585-594.

Wallace, T.F., Kremzar, M.H. (2001). ERP: Making it Happen (The Implementer's Guide to Success with Enterprise Resource Planning). New York: Wiley.

Wang, E., \& et al. (2007). Improving ERP fit to organizational process through knowledge transfer. International Journal of Information Management, 27, 200212.

Wei, C. (2008). Evaluating the performance of an ERP system based on the knowledge of ERP implementation objectives. International Journal of Advanced Manufacturing Technology, 39, 168-181.

Wei, C., Chien, C., \& Wang, M. (2005). An AHP-based approach to ERP system selection. Int. J. Production economics, 96, 47-62.

Whitten, J.L., Bentley, L.D., \& Dittman, K.C. (2001). Systems Analysis and Design Methods. New York, NY: McGraw-Hill Higher Education.

Wu, J. (2008). Critical Success Factors for ERP System Implementation. In Research and Practical Issues of Enterprise Information Systems II Volume 1 (Vol. 254, pp. 739-745). Boston: Springer.

Yazgan, H., Boran, S., \& Goztepe, K. (2009). An ERP software selection process with using artificial Neural Network based on analytical network process approach. Expert Systems with Applications, 36, 9214-9222.

Yazgan, H.R., Boran,S., Goztepe,K. (2009). An ERP software selection process with using artificial neural network based on analytic network process approach. Expert Systems with Applications, 36, 9214-9222.

Yen, H.R., \& Sheu,C. (2004). Aligning ERP implementation with competitive priorities of manufacturing firms: an exploratory study. International Journal of Production Economics, 92, 207-220.

Yusuf, Y., Gunasekaran, A., \& Althorpe, M.S. (2004). International Journal of Production Economics, 87(3), 251-266.

Zaitun, A. Z. (2008). A web-based DSS for the evaluation of an ERP system. Proceedings of iiWAS2008 (pp. 698-701). Linz: ERPAS. 


\title{
APPENDICES
}

\author{
Appendix A:
}

Thomann's Meta-methodology - Draft VI

I. Put the methodologist in touch with the problem.

a. Simple method - Use the interest of the methodologist

b. Complex method - Use Coffing Client Demand methodology

II. Purpose of the Methodology

a. Investigate the problem area

1.Read the Literature

2.Talk to people in work area

3.Examine work being done in the area.

4.Brainstorm about the problem area

5.Try out tools that already exist in the area

b. Narrow the area into manageable pieces

c. Investigate purposes that will solve the chosen piece within the problem area.

1.Brainstorm purposes that will solve the chosen problem

2.Read the literature applicable to the chosen problem

3.Ask others for purposes they think will solve the problem

d. If more than one purpose has resulted from the previous step, then chose the most appropriate one.

e. Check the chosen purpose against the following two criteria

1.Check purpose to see if it is not trivial

2.Check purpose to see if it really solves the problem

3.If purpose fails either of these criteria revise until it meets them both

f. If resources warrant show purpose to others for their critique base on the above two criteria. 
g. Write out the purpose and commit yourself to it. (If you can say why you don't like it, recycle to II e; if not go to step III).

III. Test the Purpose of the Methodology

a. Is purpose desirable?

1. Use one method

a. Simple method

i. Researcher response.

ii. Group response.

b. Complex method - Use Coffing Client Demand methodology

2. Revise purpose if necessary.

b. Is purpose operationalizable?

1. Use operationalization of fuzzy concepts. N.B. It is not necessary to completely operationalize the purpose at this point. It is only necessary to find out if the purpose can be operationalized.

2. Revise purpose if necessary.

c. Is purpose practicable?

1. Researcher response

a. Is development of a methodology practical given purpose?

b. Is the methodology, once developed, a practical way to accomplish the purpose?

2. Group response

a. Is development of a methodology practical given purpose? (methodologists)

b. Is the methodology, once developed, a practical way to accomplish the purpose? (users and methodologists)

3. Revise purpose if necessary and recycle through a. and b. otherwise go to d. 
d. Are existing methodologies insufficient?

1. Test in the following way:

a. Search area for existing methodologies

b. Take found methodologies and test them against the definition of the methodology. If all fail - go to step IV.

c. Are they designed to accomplish the purpose? If not - go to step IV.

d. Does any one of them accomplish the purpose? If not - go to step IV.

e. Are they practical?( see if its used). If not - go to step IV.

f. Are they desirable? If not - go to step IV.

g. Is it complete? If not work on this methodology.

2. Revise the purpose and recycle through the tests if necessary.

IV. Implications of the purpose for the development of the methodology are analyzed when all of the elements of III are answered affirmatively. This serves to identify the attributes that the methodology must have.

a. Use following method to analyze implications - "Problem implies its own solutions".

1. Analysis

a) Imagine and write down ways that you can fail to accomplish the purpose.

b) Imagine and write down ways you can accomplish the purpose avoiding all the problems

c) Imagine the purpose being accomplished, write down what is happening.

d) Combined analysis 
i. For each element in $b+c$, determine all possible alternatives to accomplish the purpose.

ii. Combine two lists into one; turn alternative from i.a. around so they fit together with list from d. i..

iii. Test the completeness by using one or more of the following techniques:

1. Ask others to do steps a-c

2. Think up alternatives that have nothing to do with this purpose and consider whether they may apply

3. Revisit lists from $\mathrm{b}$ and $\mathrm{c}$ and reconsider them, or add any new ones.

4. Ask if alternatives have alternatives.

5. Ask what bad alternatives exists and consider how they should be modified to become good alternatives

6. Use any other tests you choose.

ii. If at this point you cannot choose one of the alternatives as a best alternative then;

a. Determine a value system.

b. Use value system to turn all elements of list into positive alternatives.

b. Organize the attributes into a rational order of steps.

1. Determine which implications are not necessary and strike them from list.

2. Determine which implications are combined in others, combine them 3. Ask what implications need to accomplished first to accomplish the rest.

4. Write it out as a first step 
5. Ask which implication would now be first given that the first is accomplished.

6. Write down as a second step

7. Do this until all major implications are accounted for

8 . Order the substeps by cycling through steps $3-7$

9. Check to see if it has a logical flow

10. Check to make sure all implications are stated procedurally.

11. Write out a revised list

12. Have someone else order the implications, without seeing the current list.

13. Reorder with response from 11

14. Have someone else check the new list

15. Create final ordered list, and write it out.

c. Add in any steps or functions that are implied by the existing steps at the same level of abstraction.

d. Identify anchoring steps for methodology.

1. First step - Putting methodologist in contact with problem

2. Last step - Testing if methodology worked, and recycling.

e. Write out final list to be used throughout the rest of methodology.

V. Operationalize the purpose. Use methodologists discretion -

a. If purpose is vague use operationalization of fuzzy concepts, or

b. Use straight analysis technique.

VI. Design Procedures

a. Identify the first (next) step to be designed

b. Identify the step's sub purpose.

c. Analyze implications of a sub purpose in terms of the main purpose by using procedures stated below.

1. Use the following method to analyze the implications of the sub purpose 
a) Imagine and write down ways that you can fail to accomplish the purpose.

b) Imagine and write down ways you can accomplish the purpose avoiding all the problems

c) Imagine the purpose being accomplished, write down what is happening.

d) Combined analysis

i. For each element in $b+c$, determine all possible alternatives to accomplish the purpose.

ii. Combine two lists into one; turn alternative from i.a. around so they fit together with list from d. i..

iii. Test the completeness by using one or more of the following techniques:

1. Ask others to do steps a-c

2. Think up alternatives that have nothing to do with this purpose and consider whether they may apply

3. Revisit lists from $\mathrm{b}$ and $\mathrm{c}$ and reconsider them, or add any new ones.

4. Ask if alternatives have alternatives.

5. Ask what bad alternatives exists and consider how they should be modified to become good alternatives

2. If at this point you cannot choose one of the alternatives as a best alternative then;

a. Determine a value system.

b. Use value system to turn all elements of list into positive alternatives.

b. Organize the attributes into a rational order of steps.

1. Determine which implications are not necessary and strike them from list. 
2. Determine which implications are combined in others, combine them

3. Ask what implications need to be accomplished first to accomplish the rest.

4. Write it out as a first step

5. Ask which implication would now be first given that the first is accomplished.

6. Write down as a second step

7. Do this until all major implications are accounted for

8. Order the substeps by cycling through steps $3-7$

9. Check to see if it has a logical flow

10. Check to make sure all implications are stated procedurally.

11. Have someone else order the implications, without seeing the current list.

12. Reorder with response from 11

13. Have someone else check the new list

14. Create final ordered list, and write it out.

c. Add in any steps or functions that are implied by the existing steps at the same level of abstraction.

d. Identify anchoring steps for methodology.

15. First step - Putting methodologist in contact with problem

16. Last step - Testing if methodology worked, and recycling.

e. Write out final list to be used throughout the rest of methodology.

d. Determine the amount of completeness and test for it.

e. Examine the logic of the step under design in terms of subpurpose and main purpose.

f. Fill the gaps found in VI e and recycle the step. If no gaps remain go to VI g. 
g. Examine the logic of the entire methodology and its parts in terms of main purpose in light of the step under development.

h. Redesign step and/or methodology and recycle VI g. If no gaps exist then go to VI i.

i. Recycle through VI a. until it is felt that no significant improvement is being produced that warrants spending resources. Recycling to VI a. and moving on to VII a. can be done at the same time.

VII. Procedure testing and revisions to purpose/procedures

a. Field test the methodology; if necessary redesign (step VI).

b. Conclusion oriented research of methodology; if necessary redesign (step VI). 


\section{Appendix B}

Accounting Requirements:

The following accounting features were identified by $\mathrm{ABC}$ personnel as important features to have in the ERP system.

1. ERP System needs to communicate with ADP.

2. 16-17 character long for contract number is required

3. Reduced Accounts Receivable workload

4. Reduced Accounts Payable workload

5. Reduced General ledger workload

6. Accounting dashboard

7. Custom financial reporting

8. Consistency of data across reports

9. Financial report and cost tracking

10. Sales commission to be considered

11. Possibility of off-site operation. (Two different companies)

12. Generate book to bill ratios, $\mathrm{P} \& \mathrm{~L}$ report, and income and expenses report (biweekly), general \& administration cost (quarterly).

13. Interact with other systems and applications (i.e. inventory, work order tracking, work progress, and shipping).

14. Departmental profit and loss statements.

15. Determine project profitability.

16. Determine ROI.

17. Generate vouchers and invoices.

18. Ability to store and use recurring invoices.

19. Print monthly checks.

20. Track commissions, invoices.

21. Employee time sheets.

22. Earned Value Management System (EVMS)

23. Value Stream Mapping capabilities (VSM) 
Manufacturing Requirements:

The following accounting features were identified by $\mathrm{ABC}$ personnel as important features to have in the ERP system.

1. Rate suppliers upon receipts of those materials

2. Tracking product history

3. Configuration Management

4. Set up Job Codes, Set Start and End of Job Quotes

5. ERP should accommodate all Manufacturing functions, Operations, RMA, Shop Floor Control, Inventory Control, Material Access Manager

6. Bill of Materials need to control affectivity dates

7. Need ability to track inventory

8. Ability to accept inventory under different activities

9. Needs to be able to specified if inventory is government owned

10. Provide better inside in labor

11. Cost variance

12. Material Management

13. Tracking material and engineering hours for design methods

14. Needs to track cost on current working piece

15. Manage Resource planning

16. Product data Management

17. BOM pricing (track date of pricing)

18. Track defects for parts per million

19. Vendor History - Pricing, Delivery

20. BOM Revisions

21. BOMs should have links to the supporting documents (Diagrams, Schematic, ECOs, etc.)

22. SPC Measurements

23. Automated the traveler

24. More control on inventory consumption 
25. Production control planning

26. Work Order control

27. More robust scenarios for Planning

28. Give user opportunity to look at schedule change

29. Need to be able to look at a part number and see inventory requirements and order it in lots. How long is that part going to last?

30. Back flush completion in Work Order

31. Allow for category work

32. Inventory Tracking (ABC Property, Government Property)

33. Standardized tool to generate quotes.

34. Ability to change profit margin rates and override alerts

35 . Ability to track quotes and follow up orders.

36. Ability to track prior quotes on same orders.

37. Tracking manufacturing projects status in real time

38. Track model numbers and serial numbers

39. Track failed parts, test failures.

40. Track scrap cost, Engineering change orders, tooling

Other Requirements:

The following are additional features proposed by $\mathrm{ABC}$ personnel as important to have in the ERP system.

1. Research \& Development areas need to be represented in ERP

2. Need Project Planning / Scheduling

3. Need to account for time and material for federal projects

4. Bill of Material - accommodating coding related to issues of AS9100

5. Accommodate quoting

6. Emphasis on the importance to control documentations

7. System that easy to manage and administer

8. System needs to be easy to integrate

9. Dashboard (Integrated pictures of everything)

10. Track training hours 
11. Automated Requisition System - Email notification. Electronic Signatures

12. Bar Code Optional but desirable

13. Multiple companies with same pages. $\mathrm{ABC}$ may require that another company may be setup in order to share revenue/cost etc.

14. Track products station to station

15. Import/Export coding.

16. Tracking a rating suppliers (List Preferred suppliers, reporting late deliveries)

17. Need to facture in the package ability to update Small Systems to allow those minor reports, etc

18. Permission to allow user control access to the system or to areas of the system

19. Communication with ACT Software will be beneficial

20. Human Resource Functions

Reports Needed and Reporting Feature Comments:

1. MRP system should allow for Reporting, Matrix

2. ERP needs to offer User Friendly, History, Performance Standard, Respond Time, Reporting

3. Cost performance reports: EAC, ETC variances. A view of each and every plan as it comes along.

4. Cost schedule report

5. Reports for accounting : budget comparison, track labor by hours/dollars, material cost, no quantity in Peachtree only dollars,

6. Monthly based - analysis to upper management analysis on every programs.

7. Customer Reporting / Upper Management / Rate Calculation /

8. Direct labor hour allocation and fulfillment,

9. Cost of Bill of Material

10. Detail vendor information

11. AP aging detail information

12. PO commitment report

13. ABC Admin will like to see a integrated picture of everything

14. Paying vendors, Paying APs, Check runs 
15. Exception reports - what parts need to push in and what needs to be push out

16. PO Commitment report capability - To see what's pending

17. Requisition - Need to see what's the demand, what's on order, this is when we are going out of order.

18. Exception Reports on Requisitions

19. Purchase History Report, Lead Time and Cost (For audit validation)

20. Easy to use report that will demonstrate: What's shipping this week?

21. Gross Margin Percentage report

22. Revenue forecasting

23. Reconciliation in payroll to see annual salary, monthly salary, actual salary. To easily see what are the differences.

Expectations and Benefits Sought:

a. The expectations of the new or improved systems were:

- ERP System that will integrate data management throughout the enterprise

- Ability to track physical shipments to measure the success of the business.

- Ability to integrate accounting, and manufacturing.

- A system that can handle the company's growth and flexibility.

- Better control over inventory.

- Ability to prepare income and expense reports internally i.e. independent of the accountant.

- Improve accuracy of the general ledger.

- Streamline quotation, payments, and invoicing, issuing checks.

- Timely billing.

- Ease of ROI calculations.

- Collect the test data and represent the test results in the form of charts.

b. Benefits sought with the implementation of a new system:

- Improve efficiency of financial data entry

- Improve reliability of data across reports and user functions

- Improve efficiency/ timeliness of financial reporting

- Improve consistency of financial reporting 
- Provide EVMS at project level

- Provide Value stream mapping capability

- DCAA acceptable system

- Improve efficiency of financial data entry

- Improve overall business data management.

- Establish inventory management system.

- Reduce overhead.

- Reduce throughput time.

- Reduce manual reporting.

- Improve productivity.

- An integrated management of accounting and manufacturing. 
Appendix C

Engineering Design and Software Tools Used/Needs at ABC - April 2009

Systems Engineering:

For requirements management purposes, $\mathrm{ABC}$ uses spreadsheet methods, but does not have DOORS like software tools.

QuART Professional Reliability Prediction Software

ABC uses this tool for -217 reliability analysis. The QuART software series represents the fourth generation of the "Reliability Toolkit" series of RMQ engineering aids originated in 1988 by Quanterion personnel while working at the Rome Laboratory (formerly Rome Air Development Center). The QuART products are intended to be desktop sets of tools for practicing RMQ professionals, as well as others with an interest in the value of RMQ in products and systems.

Mechanical Engineering:

SolidWorks (SW)

Used as a comprehensive 3-D design software. ABC needs to upgrade to SW Simulation Premium for FEA/Thermal Analysis capabilities to replace a no longer supported version of COSMOS/M. SW is ABC's primary 3-D design tool. ABC had prior experience with Pro-E but had not used it in several years.

Auto-CAD

Auto-Cad software is 2-D and 3-D design visualization software; it is ABC's primary basic design tool.

\section{COSMOS/M}

COSMOS/M has a variety of bundles and option configurations, seen as cost effective because the company only pays for the functionalities it needs whether your analysis requires linear static, frequency buckling, thermal, shell analysis, assemblies capabilities, nonlinear and fatigue analysis. ABC's version of COSMOS had expired and no longer supported.

Electrical Engineering:

ICAP/4 Test Designer

The electronics design team is the primary user of ICAP/4. ABC has two seats. Test Designer takes ICAP/4's most abundant analog, mixed-signal and mixed-engineering systems simulation offering (ICAP/4 Professional), and adds in full test diagnosis for isolating faults across designs. Several methods can be used to specify pass/fail criteria for a wide variety of prescribed measurements at design nodes and in components (i.e., user-prescribed limits, derivation from lab measurements and 3-sigma range obtained from Monte Carlo statistical run). Extensive fault injection is then performed throughout the design, which automatically injects fault conditions (i.e., open, short, stuck-at value) in components. A fault universe is automatically generated for all fault conditions. A weighted test-sequencing algorithm is run, which reports detected faults and quality of 
fault coverage. Test Designer also generates pseudo test code for export to automated test equipment.

\section{ORCAD pSPICE}

Provides rapid, complete simulation of analog/mixed-signal circuits. Advanced Analysis prevents board failures by determining which components are over-stressed using Smoke analyses or observing component yields using Monte Carlo analysis. Single-button simulation, cross-probing, and full integration with ORCAD Capture improves productivity and data integrity.

Silvaco Tools

These are specialized tools for IC design. Team leader and one other engineer are the primary users of Silvaco tools which include:

Gateway - Schematic capture, Design layout

SmartSpice - Circuit simulation

Guardian DRC - Design rule check

Guardian LPV - Layout versus schematic

Guardian LPE - Layout parasitic extraction

Silos - Verilog simulator

Expert - Layout editor

Harmony - Analog mixed-signal simulator

\section{PowerWise WEBENCH}

The WEBENCH environment provides the end-to-end design and prototyping needed to create power supplies or DC-DC Converters that meet your design requirements. WEBENCH tools enable designers to solve switching power supply design problems before a prototype is built, alleviating the time and trouble associated with traditional design methods. The WEBENCH toolset can also be used as a device selection tool to find the best voltage regulator, switching regulator, switched capacitor, mosfet controller, or LDO for your application or power supply solution.

\section{Linear Technology SwitcherCAD}

SwitcherCAD III is the third generation switching regulator design program. The program consists of a high performance SPICE simulator extended with a mixed mode simulation capability that includes new intrinsic SPICE devices for macromodelling Switch Mode Power Supply (SMPS) controllers and regulators. The program includes an integrated hierarchical schematic capture program that allows users to edit example SMPS circuits or design new circuits. An integrated waveform viewer displays the simulated waveforms and allows further analysis of the simulation data. There is a builtin database for most of Linear Technology's power ICs and many passive components. The device database, schematic editing, simulation control and waveform analysis are integrated into one program. Due to the mixed mode simulation capability and many other enhancements over previous SPICE programs, the simulation speed is greatly improved while simulation accuracy is retained. Detailed cycle-by-cycle SMPS simulations can be performed and analyzed in minutes. A user can get a detailed analysis of power systems with a few mouse clicks without knowing anything about the device, 
SPICE or the schematic capture program. Synthesized or pre-drafted demo circuits can be used as a starting point to build the custom circuit to fit different power supply requirements. After the new schematic is created, the system can be simulated and a report generated. The program's integrated hierarchical schematics capture information for SPICE so that the simulator is completely available for general use. The improved performance of the SPICE simulation engine is a benefit for simulating general analog circuits and is used by all electronic engineers. With over 100,000 copies distributed so far, many users have reported that LTspice/SwitcherCAD III is their main simulation/schematic capture tool. The program is an industry benchmark.

IBIS Interface Models

IBIS (Input/Output Buffer Information Specification) is a behavioral model in ASCII file format that describes the input/output characteristics of a device. The IBIS specification provides a way for $\mathrm{I} / \mathrm{V}$ and $\mathrm{V} / \mathrm{T}$ data of a device to be used in simulators for signal integrity analysis. The IBIS specification was developed so that semiconductor manufacturers could provide models of their devices with fast simulation times, multiplatform support, and without sharing intellectual property (circuit design and process), such as the case with SPICE models. Since an IBIS file is a behavioral model, it also has the benefit of simulating at much faster times than a SPICE model file. The IBIS specification is a recognized standard, ANSI/EIA-656A.

Software/Embedded Design Engineering:

General Simulation and Modeling Tools

- Visual Basic

- Visual $\mathrm{C}++$

- MathCAD

- IDL

- PV-Wave Command Language

- MatLab

- Simulink

GUI/Console Development Environments

- Lab VIEW

- QT Cross-Platform application development framework for GUI development, and non-GUI programs such as console tools and servers.

- Visual Basic/C++

Micro-Controller Development Tools at ABC

- S08 MCU

- ARM9 Embedded Microprocessor

- Blackfin Processor 
Manufacturing:

EZMRP \& Pulse

An in-house developed MRP and Digital Dashboard Software tool suite used at ABC. $\mathrm{ABC}$ needs a more robust, ERP tool for mid-level manufacturing and product development enterprise. Financial system, job tracking, job costing, production and inventory, and program management suites are of priority interest. Financials, job costing/tracking, inventory are first priorities.

Rockwell Automation Arena Production Planning modeling and simulation tool Arena Basic simulation software is most effective when modeling and analyzing business, service, or (not material handling intensive) manufacturing processes or flows. Provides an analysis of simple (non-material handling intensive) manufacturing process flows. ABC primarily uses this tool for production planning sections in large quantity/flow production proposals.

Product Data Management:

ABC uses MS Excel®... from proposal BOM, to engineering BOMs and Parts Lists, to first production stages. These are manually released to $\mathrm{CM}$ for control at that point. When product data is matured for production, it is imported into our EZMRP. This should be improved to an automated PDM in the future that is more tightly integrated into the ERP. 


\section{Appendix D}

\section{A. Candidate Software Vendors - Showing Users by Revenue Class}

\begin{tabular}{|c|c|c|c|c|c|}
\hline & & \multicolumn{4}{|c|}{ Annual Gross Revenue } \\
\hline Company & Product & $\begin{array}{l}\$ 30-70 \\
\text { MILLION }\end{array}$ & $\begin{array}{l}\$ 10-30 \\
\text { MILLION }\end{array}$ & $\begin{array}{l}\text { less than } \\
\$ 10 \text { million }\end{array}$ & $\begin{array}{l}\text { Total } \\
\text { Revenue } \\
\text { Rank }\end{array}$ \\
\hline Automated Computer Solutions & Pro:Man & 3 & 3 & 2 & 8 \\
\hline Expandable Software Inc. & Expandable & 2 & 2 & 2 & 6 \\
\hline Open Source (Free Software) & PostBooks ERP & 2 & 2 & 3 & 7 \\
\hline Consona Corporation & Relevant ERP & 2 & 3 & 1 & 6 \\
\hline Exact Software & Macola ES & 3 & 3 & 2 & 8 \\
\hline Exact Software & MAXERP & 2 & 3 & 1 & 6 \\
\hline IFS North America Inc. & IFS Applications & 2 & 1 & 0 & 3 \\
\hline IT3, Inc. & IT3 Workspace & 1 & 2 & 2 & 5 \\
\hline MISys Inc. & MISys Manufacturing System & 1 & 3 & 3 & 7 \\
\hline Pentagon 2000 Software Inc. & Pentagon 2000 & 2 & 2 & 1 & 5 \\
\hline Bowen \& Groves Inc. & M1 & 1 & 3 & 3 & 7 \\
\hline Epicor Software Corporation & Vantage & 3 & 2 & 1 & 6 \\
\hline Exact Software & Alliance/MFG & 1 & 1 & 3 & 5 \\
\hline Global Shop Solutions & Global Shop Solutions & 2 & 3 & 2 & 7 \\
\hline Horizons International Inc. & Horizons Mfg. Suite & 2 & 3 & 1 & 6 \\
\hline Infor & Infor ERP SL & 3 & 3 & 1 & 7 \\
\hline \begin{tabular}{|l} 
Jaas Systems \\
\end{tabular} & JAMS & 2 & 2 & 2 & 6 \\
\hline ManEx Inc. & Manex ERP & 1 & 3 & 3 & 7 \\
\hline Microsoft Business Solutions & Microsoft Dynamics GP & 2 & 3 & 2 & 7 \\
\hline Cincom Systems Inc. & CONTROL & 1 & 1 & 0 & 2 \\
\hline Consona Corporation & Made2Manage ERP & 1 & 3 & 3 & 7 \\
\hline CoSs Systems, Inc. & COSS Manufacturing & 2 & 3 & 1 & 6 \\
\hline Enhanced Systems \& Services Inc. & Finesse ERP & 3 & 3 & 0 & 6 \\
\hline Infor & Infor ERP COM & 3 & 1 & 1 & 5 \\
\hline Infor & Infor ERP TRANS4M & 3 & 1 & 0 & 4 \\
\hline Manufacturing Action Group Inc. & WinMAGI & 2 & 3 & 2 & 7 \\
\hline Microsoft Business Solutions & Microsoft Dynamics NAV & 2 & 3 & 2 & 7 \\
\hline OmegaCube Technologies & PowerERP & 2 & 3 & 3 & 8 \\
\hline Oracle Corporation & Oracle Applications (including JD Edwards) & 1 & 0 & 0 & 1 \\
\hline Positive Business Solutions Inc. & Profit Manager & 2 & 3 & 3 & 8 \\
\hline
\end{tabular}




\section{B. Candidate Software Vendors - Showing Users by Industry Sector.}

\begin{tabular}{|c|c|c|c|c|c|}
\hline & & \multicolumn{4}{|c|}{ Industry } \\
\hline Company & Product & $\begin{array}{l}\text { Aerospace } \\
\text { \& Defence } \\
\text { Manufacturing }\end{array}$ & $\begin{array}{l}\text { Electronic } \\
\text { Components } \\
\text { Manufacturing }\end{array}$ & $\begin{array}{l}\text { Electronic } \\
\text { Equipment } \\
\text { Manufacturing }\end{array}$ & $\begin{array}{l}\text { Electronic } \\
\text { Semi-conductor } \\
\text { manufacturing }\end{array}$ \\
\hline Automated Computer Solutions & Pro:Man & 2 & 0 & 3 & 0 \\
\hline Expandable Software Inc. & Expandable & 1 & 1 & 3 & 2 \\
\hline Open Source (Free Software) & PostBooks ERP & 1 & 2 & 1 & 1 \\
\hline Consona Corporation & Relevant ERP & 3 & 1 & 1 & 1 \\
\hline Exact Software & Macola ES & 0 & 1 & 1 & 0 \\
\hline Exact Software & MAXERP & 1 & 2 & 2 & 1 \\
\hline IFS North America Inc. & \begin{tabular}{|l|} 
IFS Applications \\
\end{tabular} & 2 & 2 & 2 & 1 \\
\hline IT3, Inc. & IT3 Workspace & 0 & 3 & 3 & 0 \\
\hline MISys Inc. & MISys Manufacturing System & 0 & 1 & 2 & 1 \\
\hline Pentagon 2000 Software Inc. & Pentagon 2000 & 3 & 1 & 2 & 0 \\
\hline Bowen \& Groves Inc. & M1 & 1 & 1 & 1 & 1 \\
\hline Epicor Software Corporation & Vantage & 2 & 1 & 2 & 0 \\
\hline Exact Software & Alliance/MFG & 1 & 1 & 3 & 0 \\
\hline Global Shop Solutions & Global Shop Solutions & 1 & 1 & 1 & 1 \\
\hline Horizons International Inc. & Horizons Mfg. Suite & 0 & 1 & 2 & 0 \\
\hline Infor & Infor ERP SL & 1 & 1 & 1 & 0 \\
\hline Jaas Systems & JAMS & 1 & 1 & 2 & 0 \\
\hline ManEx Inc. & Manex ERP & 0 & 1 & 3 & 0 \\
\hline Microsoft Business Solutions & Microsoft Dynamics GP & 0 & 1 & 1 & 1 \\
\hline Cincom Systems Inc. & CONTROL & 3 & 1 & 1 & 1 \\
\hline Consona Corporation & Made2Manage ERP & 0 & 2 & 0 & 0 \\
\hline COSS Systems, Inc. & coss Manufacturing & 2 & 1 & 1 & 0 \\
\hline Enhanced Systems \& Services Inc. & Finesse ERP & 3 & 0 & 0 & 0 \\
\hline Infor & Infor ERP COM & 1 & 1 & 1 & 0 \\
\hline Infor & Infor ERP TRANS4M & 1 & 2 & 1 & 0 \\
\hline Manufacturing Action Group Inc. & WinMAGI & 2 & 0 & 0 & 0 \\
\hline \begin{tabular}{|l|} 
Microsoft Business Solutions \\
\end{tabular} & Microsoft Dynamics NAV & 0 & 1 & 1 & 0 \\
\hline OmegaCube Technologies & PowerERP & 0 & 0 & 2 & 0 \\
\hline Oracle Corporation & Oracle Applications (including JD Edwards) & 3 & 1 & 2 & 2 \\
\hline Positive Business Solutions Inc. & Profit Manager & 0 & 2 & 1 & 0 \\
\hline
\end{tabular}




\section{Candidate Software Vendors - Showing Users by Revenue Class.}

\begin{tabular}{|c|c|c|c|c|c|c|}
\hline \multirow[b]{2}{*}{ Company } & \multirow[b]{2}{*}{ Product } & \multicolumn{4}{|c|}{ Manufacturing Environment } & \multirow[b]{2}{*}{ Grand Total } \\
\hline & & $\begin{array}{l}\text { Contract/ Project } \\
\text { Manufacturing }\end{array}$ & $\begin{array}{l}\text { Process/ Batch } \\
\text { Manufacturing }\end{array}$ & $\begin{array}{l}\text { Work-order } \\
\text { driven }\end{array}$ & $\begin{array}{l}\text { Total Manufacturing } \\
\text { Environment }\end{array}$ & \\
\hline Automated Computer Solutions & Pro:Man & 3 & 1 & 3 & 7 & 7 \\
\hline Expandable Software Inc. & Expandable & 1 & 1 & 3 & 5 & 5 \\
\hline Open Source (Free Software) & PostBooks ERP & 1 & 1 & 3 & 5 & 5 \\
\hline Consona Corporation & Relevant ERP & 3 & 0 & 2 & 5 & 5 \\
\hline Exact Software & Macola ES & 1 & 2 & 3 & 6 & 6 \\
\hline Exact Software & MAXERP & 0 & 1 & 3 & 4 & 4 \\
\hline IFS North America Inc. & IFS Applications & 2 & 1 & 3 & 6 & 6 \\
\hline IT3, Inc. & IT3 Workspace & 0 & 2 & 3 & 5 & 5 \\
\hline MISys Inc. & MISys Manufacturing System & 1 & 1 & 3 & 5 & 5 \\
\hline Pentagon 2000 Software Inc. & Pentagon 2000 & 2 & 1 & 2 & 5 & 5 \\
\hline Bowen \& Groves Inc. & M1 & 1 & 0 & 3 & 4 & 4 \\
\hline Epicor Software Corporation & Vantage & 1 & 0 & 3 & 4 & 4 \\
\hline Exact Software & Alliance/MFG & 1 & 1 & 3 & 5 & 5 \\
\hline Global Shop Solutions & Global Shop Solutions & 1 & 0 & 3 & 4 & 4 \\
\hline Horizons International Inc. & Horizons Mfg. Suite & 1 & 2 & 3 & 6 & 6 \\
\hline Infor & Infor ERP SL & 1 & 1 & 3 & 5 & 5 \\
\hline Jaas Systems & JAMS & 2 & 0 & 3 & 5 & 5 \\
\hline ManEx Inc. & Manex ERP & 1 & 0 & 3 & 4 & 4 \\
\hline Microsoft Business Solutions & Microsoft Dynamics GP & 1 & 1 & 3 & 5 & 5 \\
\hline Cincom Systems Inc. & CONTROL & 3 & 1 & 2 & 6 & 6 \\
\hline Consona Corporation & Made2Manage ERP & 1 & 1 & 3 & 5 & 5 \\
\hline CoSs Systems, Inc. & COSS Manufacturing & 2 & 1 & 1 & 4 & 4 \\
\hline Enhanced Systems \& Services Inc. & Finesse ERP & 3 & 0 & 2 & 5 & 5 \\
\hline Infor & Infor ERP COM & 2 & 1 & 3 & 6 & 6 \\
\hline Infor & Infor ERP TRANS4M & 2 & 1 & 3 & 6 & 6 \\
\hline Manufacturing Action Group Inc. & WinMAGI & 1 & 1 & 3 & 5 & 5 \\
\hline Microsoft Business Solutions & Microsoft Dynamics NAV & 1 & 1 & 3 & 5 & 5 \\
\hline OmegaCube Technologies & PowerERP & 1 & 1 & 2 & 4 & 4 \\
\hline Oracle Corporation & Oracle Applications (including JD Edwards) & 2 & 2 & 1 & 5 & 5 \\
\hline
\end{tabular}




\section{Appendix E}

\begin{tabular}{|c|c|c|c|c|}
\hline & \multicolumn{2}{|l|}{ SUMMARY OF ABC ERP REQUIREMENTS } & & \multirow[b]{2}{*}{$\begin{array}{l}\text { Not very } \\
\text { important }\end{array}$} \\
\hline & & $\begin{array}{l}\text { Extremely } \\
\text { Important }\end{array}$ & & \\
\hline & Rate Requirements as follows & 10 & $\ldots \ldots \ldots$ & 1 \\
\hline & & & & \\
\hline & Accounting Requirements & & & \\
\hline 1 & ERP System needs to communicate with ADP. & & 10 & \\
\hline 2 & 16-17 character long for contract number is required & & 10 & \\
\hline 3 & Reduced Accounts Payable workload & & 10 & \\
\hline 4 & Reduced General ledger workload & & 10 & \\
\hline 5 & Custom financial reporting & & 10 & \\
\hline 6 & Sales commission to be considered & & 10 & \\
\hline 7 & Possibility of off-site operation. (Two different companies) & & 10 & \\
\hline 8 & Departmental profit and loss statements. & & 10 & \\
\hline 9 & Determine project profitability. & & 10 & \\
\hline 10 & Generate vouchers and invoices. & & 10 & \\
\hline 11 & Print monthly checks. & & 10 & \\
\hline 12 & Track commissions, invoices. & & 10 & \\
\hline 13 & Earned Value Management System (EVMS) & & 10 & \\
\hline 14 & Value Stream Mapping capabilities (VSM) & & 10 & \\
\hline 15 & Government Cost Account (GCA)- Timekeeping (time by project) & & 10 & \\
\hline 16 & GCA - Project Costing (tracking of all materials and labor by project) & & 10 & \\
\hline 17 & GCA - Multiple Overhead Pools \& one G\&A & & 10 & \\
\hline 18 & GCA - Time and Material (Revenue and Billing) & & 10 & \\
\hline 19 & $\begin{array}{l}\text { GCA - Cost - must calculate revenue on labor \& materials using actual } \\
\text { direct costs plus Billing Overhead + Billing G\&A rate by year }\end{array}$ & & 10 & \\
\hline 20 & GCA - FFP - system must accommodate milestone billing on projects & & 10 & \\
\hline 21 & Reduced Accounts Receivable workload & & 9 & \\
\hline 22 & Accounting dashboard & & 8.5 & \\
\hline 23 & $\begin{array}{l}\text { Interact with other systems and applications (i.e. inventory, work order } \\
\text { tracking, work progress, and shipping). }\end{array}$ & & 8 & \\
\hline 24 & Determine ROI. & & 7 & \\
\hline 25 & Financial report and cost tracking & & 6 & \\
\hline 26 & Ability to store and use recurring invoices. & & 5 & \\
\hline 27 & Consistency of data across reports & & 3 & \\
\hline 28 & Employee time sheets. & & 3 & \\
\hline 29 & $\begin{array}{l}\text { Generate book to bill ratios, P \& L report, and income and expenses } \\
\text { report (biweekly), general \& administration cost (quarterly). }\end{array}$ & & 2 & \\
\hline
\end{tabular}




\begin{tabular}{|c|c|c|c|c|}
\hline & \multicolumn{2}{|l|}{ SUMMARY OF ABC ERP REQUIREMENTS } & & \multirow[b]{2}{*}{$\begin{array}{l}\text { Not very } \\
\text { important }\end{array}$} \\
\hline & & $\begin{array}{l}\text { Extremely } \\
\text { Important }\end{array}$ & & \\
\hline & Rate Requirements as follows & 10 & $\ldots \ldots \ldots$ & 1 \\
\hline & Manufacturing Requirements & & & \\
\hline 1 & Rate suppliers upon receipts of materials & & 10 & \\
\hline 2 & Tracking product history & & 10 & \\
\hline 3 & Set up Job Codes, Set Start and End of Job Quotes & & 10 & \\
\hline 4 & $\begin{array}{l}\text { ERP should accommodate all Manufacturing functions, Operations, } \\
\text { RMA, Shop Floor Control, Inventory Control, Material Access } \\
\text { Manager }\end{array}$ & & 10 & \\
\hline 5 & Need ability to track inventory & & 10 & \\
\hline 6 & Provide better insight in labor & & 10 & \\
\hline 7 & Cost variance & & 10 & \\
\hline 8 & Needs to track cost on current working piece & & 10 & \\
\hline 9 & Manage Resource planning & & 10 & \\
\hline 10 & BOM pricing (track date of pricing) & & 10 & \\
\hline 11 & Vendor History - Pricing, Delivery & & 10 & \\
\hline 12 & BOM Revisions & & 10 & \\
\hline 13 & SPC Measurements & & 10 & \\
\hline 14 & Automate the traveler & & 10 & \\
\hline 15 & More control on inventory consumption & & 10 & \\
\hline 16 & Production control planning & & 10 & \\
\hline 17 & Work Order control & & 10 & \\
\hline 18 & More robust scenarios for Planning & & 10 & \\
\hline 19 & Give user opportunity to look at schedule change & & 10 & \\
\hline 20 & Back flush completion in Work Order & & 10 & \\
\hline 21 & Allow for category work & & 10 & \\
\hline 22 & Standardized tool to generate quotes. & & 10 & \\
\hline 23 & Ability to change profit margin rates and override alerts & & 10 & \\
\hline 24 & Ability to track quotes and follow up orders. & & 10 & \\
\hline 25 & Ability to track prior quotes on same orders. & & 10 & \\
\hline 26 & Tracking manufacturing projects status in real time & & 10 & \\
\hline 27 & Track model numbers and serial numbers & & 10 & \\
\hline 28 & Configuration Management & & 9 & \\
\hline 29 & Bill of Materials need to control affectivity dates & & 8.5 & \\
\hline 30 & Tracking material and engineering hours for design methods & & 8 & \\
\hline 31 & Product Data Management & & 7 & \\
\hline 32 & Needs to be able to specified if inventory is government owned & & 6 & \\
\hline 33 & Track defects for parts per million & & 5 & \\
\hline 34 & $\begin{array}{l}\text { Be able to look at a part number and see inventory requirements and } \\
\text { order it in lots. }\end{array}$ & & 5 & \\
\hline 35 & Track failed parts, test failures. & & 5 & \\
\hline 36 & Track scrap cost, Engineering change orders, tooling & & 5 & \\
\hline 37 & Ability to accept inventory under different activities & & 3 & \\
\hline 38 & $\begin{array}{l}\text { BOMs should have links to the supporting documents (Diagrams, } \\
\text { Schematic, ECOs, etc) }\end{array}$ & & 3 & \\
\hline 39 & Inventory Tracking (CME Property, Government Property) & & 3 & \\
\hline 40 & Material Management & & 2 & \\
\hline
\end{tabular}




\begin{tabular}{|c|c|c|c|c|}
\hline \multicolumn{3}{|c|}{ SUMMARY OFABC ERP REQUIREMENTS } & & \multirow[b]{2}{*}{$\begin{array}{l}\text { Not very } \\
\text { important }\end{array}$} \\
\hline & & $\begin{array}{l}\text { Extremely } \\
\text { Important }\end{array}$ & & \\
\hline & Rate Requirements as follows & 10 & $\ldots \ldots \ldots$ & 1 \\
\hline & Other Requirements & & & \\
\hline 1 & Need Project Planning / Scheduling & & 10 & \\
\hline 2 & Need to account for time and material for federal projects & & 10 & \\
\hline 3 & Emphasis on the importance to control documentations & & 10 & \\
\hline 4 & System that easy to manage and administer & & 10 & \\
\hline 5 & Dashboard (Integrated pictures of everything) & & 10 & \\
\hline 6 & $\begin{array}{l}\text { Automated Requisition System - Email notification. Electronic } \\
\text { Signatures }\end{array}$ & & 10 & \\
\hline 7 & Bar Code Optional but desirable & & 10 & \\
\hline 8 & Track products station to station & & 10 & \\
\hline 9 & Import/Export coding. & & 10 & \\
\hline 10 & $\begin{array}{l}\text { Tracking a rating suppliers (List Preferred suppliers, reporting late } \\
\text { deliveries) }\end{array}$ & & 10 & \\
\hline 11 & $\begin{array}{l}\text { Need to facture in the package's ability to update system to allow those } \\
\text { minor reports changes }\end{array}$ & & 10 & \\
\hline 12 & $\begin{array}{l}\text { Permission to allow user control access to the system or to areas of the } \\
\text { system }\end{array}$ & & 10 & \\
\hline 13 & Communication with ACT Software will be beneficial & & 10 & \\
\hline 14 & Human Resource Functions & & 10 & \\
\hline 15 & Accommodate quoting & & 8 & \\
\hline 16 & System needs to be easy to integrate & & 7 & \\
\hline 17 & Research \& Development areas need to be represented in ERP & & 6 & \\
\hline 18 & Track training hours & & 5 & \\
\hline 19 & $\begin{array}{l}\text { Multiple companies with same pages. CME may require that another } \\
\text { company may be setup in order to share revenue/cost etc. }\end{array}$ & & 3 & \\
\hline 20 & Bill of Material - accommodating coding related to issues of AS9100 & & 2 & \\
\hline
\end{tabular}




\begin{tabular}{|c|c|c|c|c|}
\hline \multicolumn{3}{|c|}{ SUMMARY OF ABC ERP REQUIREMENTS } & & \multirow[b]{2}{*}{$\begin{array}{l}\text { Not very } \\
\text { important }\end{array}$} \\
\hline & & $\begin{array}{l}\text { Extremely } \\
\text { Important }\end{array}$ & & \\
\hline & Rate Requirements as follows & 10 & $\ldots \ldots \ldots$ & 1 \\
\hline & Report Needed and Reporting Feature Comments & & & \\
\hline 1 & MRP system should allow for Reporting, Matrix & & 10 & \\
\hline 2 & $\begin{array}{l}\text { Cost performance reports: EAC, ETC variances. A view of each and } \\
\text { every plan as it comes along. }\end{array}$ & & 10 & \\
\hline 3 & $\begin{array}{l}\text { Monthly based - analysis to upper management analysis on every } \\
\text { program. }\end{array}$ & & 10 & \\
\hline 4 & Customer Reporting / Upper Management / Rate Calculation & & 10 & \\
\hline 5 & Detail vendor information & & 10 & \\
\hline 6 & AP aging detail information & & 10 & \\
\hline 7 & $\begin{array}{l}\text { CME Admin will like to see a integrated picture of everything } \\
\text { (Executive Dashboard) }\end{array}$ & & 10 & \\
\hline 8 & $\begin{array}{l}\text { Exception reports - what parts need to push in and what needs to be } \\
\text { push out }\end{array}$ & & 10 & \\
\hline 9 & PO Commitment report capability - To see what's pending & & 10 & \\
\hline 10 & Exception Reports on Requisitions & & 10 & \\
\hline 11 & Purchase History Report, Lead Time and Cost (For audit validation) & & 10 & \\
\hline 12 & Easy to use report that will demonstrate: What's shipping this week? & & 10 & \\
\hline 13 & Gross Margin Percentage report & & 10 & \\
\hline 14 & Revenue forecasting & & 10 & \\
\hline 15 & $\begin{array}{l}\text { Reconciliation in payroll for annual salary, monthly salary, actual salary. } \\
\text { To easily see what are the differences. }\end{array}$ & & 10 & \\
\hline 16 & $\begin{array}{l}\text { ERP needs to offer User Friendly, History, Performance Standard, } \\
\text { Respond Time, Reporting }\end{array}$ & & 8.5 & \\
\hline 17 & Cost of Bill of Material & & 8 & \\
\hline 18 & PO commitment report & & 7 & \\
\hline 19 & $\begin{array}{l}\text { Reports for accounting : budget comparison, track labor by } \\
\text { hours/dollars, material cost, no quantity in Peachtree only dollars, }\end{array}$ & & 6 & \\
\hline 20 & Paying vendors, Paying APs, Check runs & & 5 & \\
\hline 21 & Cost schedule report & & 3 & \\
\hline 22 & Requisition - Need to see what's the demand, what's on order, etc. & & 3 & \\
\hline 23 & Direct labor hour allocation and fulfillment, & & 2 & \\
\hline
\end{tabular}


VITA

\section{RICHARD BURTON}

Born, South Woodford, Essex, England

$1969-1974$

$1974-1992$

Executive Officer, UK MOD

Various Positions,

Aerospace Maintenance Engineering

$1992-1993$

Research Assistant, Florida International University

Applied Research Center (ARC)

$1993-1995$

Project Manager FIU ARC

$1995-1998$

Program Manager FIU ARC

$1998-2003$

Associate Director FIU ARC

$2003-2010$

Director Business Programs FIU ARC

1993

Bachelor of Science Mechanical Engineering

Florida International University

1995

Master of Science Mechanical Engineering

Florida International University

2006

Master of Science Engineering Management

Florida International University

2011

Doctoral Candidate in Industrial and Systems Engineering

Florida International University

\section{PROFESSIONAL QUALIFICATIONS}

OPM3 ${ }^{\circledR}$ Certified Consultant

OPM3 ${ }^{\circledR}$ Certified Assessor

Project Management Professional ${ }^{\circledR}$

\section{PUBLICATIONS}

R. Burton, F. Desir, G. Hoo, G. Yang, Z. F. Dong, and M. A. Ebadian,

"An Experimental Investigation of the Solidification Process in a V-Shaped Sump," 1993 ASME Winter Annual Meeting, Heat and Mass Transfer in Materials Processing and Manufacturing, HTD 261, 1993, 45-53.

R. Burton, F. Desir, G. Hoo, G. Martin, G. Yang, Z. F. Dong and M. A. Ebadian, "Effects of Cooling Wall Angles on the Solidification Process in a Binary Mixture," 1994 ASME International Congress and Exposition, Thermal Processing of Materials, HTD 289, 1994, 163-170. 
R. Burton, F. Desir, G. Hoo, G. Martin, G. Yang, Z. F. Dong, and M. A. Ebadian, "The Solidification Process in a Rectangular Enclosure with Symmetric Cooling Walls," $6^{\text {th }}$ AIAA/ASME Thermophysics and Heat Transfer Conference, Transport Phenomena in Materials Processing and Manufacturing, HTD 280, 1994, 71-78.

R. Burton, G. Yang, Z. F. Dong, and M. A. Ebadian, "An Experimental Investigation of the Solidification Process in a V-Shaped Sump," International Journal of Heat and Mass Transfer, 38(13), 1995, 2383-2393.

S. DeZego, K. Jones Jr., R. Burton, G. Yang, Z. F. Dong, and M. A. Ebadian, "Effect of Casting Speed on the Continuous Casting of a Binary Solution," 1995 ASME International Mechanical Engineering Congress and Exposition. Fundamentals of Heat Transfer in Metal and Materials Processing, HTD 317-2, 1995, 535540 .

S. DeZego, K. Jones Jr., R. Burton, G. Yang, Z. F. Dong, and M. A. Ebadian, "Experimental Study of the Continuous Casting of a Binary Solution," Proceedings of the $30^{\text {th }} 1995$ National Heat Transfer Conference, Transport Phenomena in Manufacturing and Material Processing, 4, HTD 306, 1995, 105-112.

X. Zhou, J. Zhou, Z. Wang, D. McDaniel, W. Zhang and R. Burton, "Identification and Validation of Analytical Chemistry Methods for Detecting Composite Surface Contamination and Moisture - Solid-State Electrochemical Sensor Study", submitted to Electrochemical Transactions, 7/09.

R. Guduru, S. Alwarappan, C. Li, D. McDaniel, X. Zhou, R. Burton and W. Zhang, "Enhancing the sensitivity of atomic force microscopy by chemical modification of AFM tips with epoxy groups", in preparation for Langmuir.

\section{CONFERENCE PROCEEDINGS}

X. Zhou, J. Zhou, Z. Wang, D. McDaniel, W. Zhang and R. Burton, "Identification and Validation of Analytical Chemistry Methods for Detecting Composite Surface Contamination and Moisture - Solid-State Electrochemical Sensor Study", 215th ECS Meeting May 2009.

D. Persaud, D. McDaniel, R. Guduru, T. Pribanic, R. Burton and X. Zhou, "Experimental Validation of Analytical Chemistry Methods for Detecting Contaminants on Composite Surfaces", submitted to SAMPE 2010. 